\title{
Cardiomyopathy and muscle insulin resistance in type 2 diabetes : craving for sugars, or dying for fat?
}

Citation for published version (APA):

van de Weijer, T. (2013). Cardiomyopathy and muscle insulin resistance in type 2 diabetes : craving for sugars, or dying for fat? [Doctoral Thesis, Maastricht University]. Uitgeverij BOXPress. https://doi.org/10.26481/dis.20130705tw

Document status and date:

Published: 01/01/2013

DOI:

10.26481/dis.20130705tw

Document Version:

Publisher's PDF, also known as Version of record

\section{Please check the document version of this publication:}

- A submitted manuscript is the version of the article upon submission and before peer-review. There can be important differences between the submitted version and the official published version of record.

People interested in the research are advised to contact the author for the final version of the publication, or visit the DOI to the publisher's website.

- The final author version and the galley proof are versions of the publication after peer review.

- The final published version features the final layout of the paper including the volume, issue and page numbers.

Link to publication

\footnotetext{
General rights rights.

- You may freely distribute the URL identifying the publication in the public portal. please follow below link for the End User Agreement:

www.umlib.nl/taverne-license

Take down policy

If you believe that this document breaches copyright please contact us at:

repository@maastrichtuniversity.nl

providing details and we will investigate your claim.
}

Copyright and moral rights for the publications made accessible in the public portal are retained by the authors and/or other copyright owners and it is a condition of accessing publications that users recognise and abide by the legal requirements associated with these

- Users may download and print one copy of any publication from the public portal for the purpose of private study or research.

- You may not further distribute the material or use it for any profit-making activity or commercial gain

If the publication is distributed under the terms of Article $25 \mathrm{fa}$ of the Dutch Copyright Act, indicated by the "Taverne" license above, 
Cardiomyopathy and muscle insulin resistance in type 2 diabetes

Craving for sugars, or dying for fat?

Tineke van de Weijer 


\section{Promotores:}

Prof. dr. L.P.A.J. Schrauwen and Prof. dr. M.K.C. Hesselink

\section{Co-Promotor:}

Dr. V.B. Schrauwen-Hinderling

\section{Beoordelingscommissie/PhD thesis committee:}

Prof. dr. R. Mensink (voorzitter, department of Human Biology, Maastricht University Medical Center)

Prof. dr. M. de Haan (department of Radiology, Maastricht University Medical Center)

Dr. I. Jazet (department Endocrinology/Internal Medicine, Leiden University Medical Center) Prof. dr. P. Iozzo (Institute of Clinical Physiology, Pisa, Italy and Dept. of Medicine and Turku PET Centre Turku, Finland)

Prof. dr. L. de Windt (department of cardiology, Maastricht University Medical Center)

\section{(C)2013, C.H. van de Weijer}

Cover design: Proefschriftmaken.nl || Uitgeverij BOXPress

Printed \& Lay Out by: Proefschriftmaken.nl || Uitgeverij BOXPress

Published by: Uitgeverij BOXPress, 's-Hertogenbosch

ISBN 978-90-8891-655-7
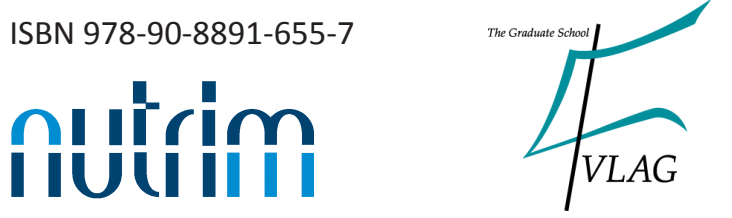

The studies presented in this thesis were performed within the NUTRIM School for Nutrition, Toxicology and Metabolism, which participates in the Graduate School VLAG, accredited by the Royal Netherlands Academy of Arts and Sciences.

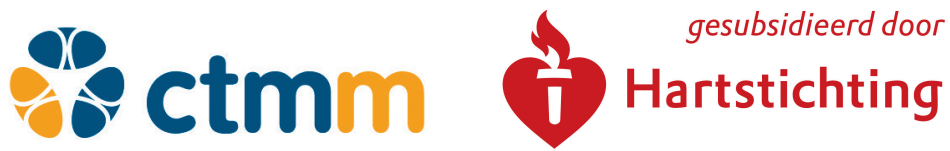

The research described in this thesis was supported by a grant of the Center for Translational Molecular Medicine (CTMM) project PREDICCt (Grant 01C-104) and the Dutch Heart Foundation. Financial Support by the Dutch Heart Foundation for the publication of this thesis is gratefully acknowledged.

All rights reserved. No part of this thesis may be reproduced or transmitted in any form or by any means, electronic or mechanical, including photocopying, recording or any information storage or recieval system, without permission in writing from the author, or, when appropriate, from the publishers of the publication. 


\title{
Cardiomyopathy and muscle insulin resistance in type 2 diabetes
}

\author{
Craving for sugars, or dying for fat?
}

\section{PROEFSCHRIFT}

Ter verkrijging van de graad van doctor aan de Universiteit Maastricht, op gezag zegging van de Rector Magnificus, Prof. dr. L.L.G. Soete, volgens het besluit van het college der Decanen,

in het openbaar te verdedigen op vrijdag 5 juli 2013 om 14.00 uur.

door

\section{Christina Helena van de Weijer}

Geboren op 31 augustus 1984 te Eindhoven, Nederland 



\section{Thesis content}

\section{Chapter 1}

page 7

General Introduction

\section{Chapter 2}

Review: Lipotoxicity in type 2 diabetic cardiomyopathy

\section{Chapter 3}

Geometrical models for cardiac MRI in rodents: comparison of quantification of left ventricular volumes and function by various geometrical models with a full-volume MRI data set in rodents

\section{Chapter 4}

ATGL-mediated fat catabolism regulates cardiac mitochondrial function via PPAR- $\alpha$ and PGC- $1 \alpha$ and $\beta$

\section{Chapter 5}

Increased intramyocellular lipids but unaltered in vivo mitochondrial oxidative phosphorylation in skeletal muscle of Adipose Triglyceride Lipase deficient mice

\section{Chapter 6}

Effects of bezafibrate treatment in a patient and a carrier with mutations in the PNPLA2 gene, causing Neutral Lipid Storage Disease with Myopathy (NLSDM)

\section{Chapter 7}

Relationships between mitochondrial function and metabolic flexibility in type 2

diabetes mellitus

\section{Chapter 8}

Exercise-induced modulation of cardiac lipid content in healthy lean young men

\section{Chapter 9}

page 185

Treatment with Acipimox fails to improve insulin sensitivity and may impair cardiac function in non-insulin dependent type 2 diabetes mellitus

\section{Chapter 10}

General discussion

\section{Summary}

Nederlandse samenvatting (Summary in Dutch)

Dankwoord

Curriculum Vitae 


\section{Diabetes; a medical history of a growing problem}

Diabetes is a disease well recognized over the ages. Although diabetes used to be a rare disease, the old Egyptian manuscripts from 1500 b.c. already mentioned a disease which was characterized by polyuria (frequent urination). The term "diabetes" was first used by the Greek physician Aretaeus who lived between 80 - 138 c.e. and means as much as passing through, referring to the polyuria seen in these patients ${ }^{1}$. The recognition of the different types of diabetes as separate conditions happened a few centuries later by two Indian physicians (Sushruta and Charaka in 400-500 a.d.). Type 1 diabetes was clearly associated with youth, whereas type 2 diabetes was associated with overweight. Although times have changed, we still use the same terminology and classification. In the last era our knowledge concerning the understanding of this disease has grown immensely and has led to better prevention and treatment of the disease. Although life expectancy and quality of life of diabetic patients has improved, ironically there are more cases of diabetes nowadays than ever before. Furthermore, with the increasing number of people being overweight, the incidence of type 2 diabetes keeps on rising and has vastly outgrown the problem of type 1 diabetes.

The latest report of the ADA of January 2011 stated that in the United States alone, 25.8 million people are suffering from diabetes. This accounts for $8.3 \%$ of the total US population. Furthermore, 1.9 million new cases of diabetes were diagnosed in the US in people aged 20 years and older in 2010, and the incidence keeps on rising every year.

Worldwide it has been estimated that in 2000 , about $2.8 \%$ of the population suffered from diabetes. It is estimated that with the recent trend in the incidence of type 2 diabetes, the prevalence of type 2 diabetes will rise to $4.4 \%$ in 2030. In elderly, the prevalence is even higher than $10 \%$. This means that the total number of people with diabetes is projected to rise from 171 million in 2000 to 366 million in $2030^{2,3}$. Although no distinction is made between the different types of diabetes, it is generally accepted that about $90 \%$ of the diabetic population suffers from type 2 diabetic patients. As the problem of type 2 diabetes clearly exceeded the problem of type 1 diabetes, this thesis will focus on the pathology of type 2 diabetes.

\section{Type 2 diabetes; resisting insulin}

Although diabetes has been recognized since antiquity, the basic pathogenesis of diabetes has only started to be unraveled experimentally since about 1900. The discovery of a central role for insulin, only came after the discovery of a role for the pancreas in diabetes by Joseph von Mering and Oskar Minkowski (1889). Insulin (derived from the Latin insula; reference 
to the islets of Langerhans) is produced by the beta-cells of the Islets of Langerhans in the pancreas and is the primary hormone responsible for the uptake of glucose from plasma by various tissues.

Under normal circumstances, elevated plasma glucose levels (for instance after a meal, or an injection or infusion of glucose) triggers the release of insulin form the pancreas. Under normal physiological conditions the release of insulin results in uptake of glucose in insulin-sensitive cells, hence reducing plasma glucose levels. In order to achieve this, insulin will bind the insulin receptors on a cell and will activate the insulin-signaling cascade, eventually resulting in translocation of the predominant glucose transporter 4 (GLUT4) to the cell membrane. These glucose transporters fuse with cellular membranes to create transmembrane domains permitting the uptake of glucose by these cells (see figure 1, for a more detailed description of the insulin signaling cascade).

In type 2 diabetes, muscle tissue (which is responsible for the major part of glucose uptake) becomes less sensitive to insulin and needs higher insulin levels for the same level of GLUT4 translocation. This mechanism is better known as 'insulin resistance'. Initially, the pancreas will compensate for the compromised insulin sensitivity, by producing more insulin. Eventually, the pancreatic insulin production will fail and insulin levels will drop, ultimately leading to elevated circulatory glucose levels. This is the moment at wich type 2 diabetes can be diagnosed ${ }^{4,5}$. The question arises how insulin resistance develops. Next to this, the relationship between insulin resistance and obesity needs to be clarified.

\section{Lipid accumulation and its role in obesity and type 2 diabetes}

As stated earlier, Indian physicians already linked obesity with type 2 diabetes as early as 400-500 a.d. This relationship has remained a hot topic throughout the ages, though a clear functional relationship was only found in the last era. In his lecture on the 1998 ADA meeting, Dr. Gerald Reaven hypothesized that the fate of obesity and type 2 diabetes might be intertwined. He proposed the theory that central obesity, diabetes and hypertension have a common cause in insulin resistance. Initially he called this "syndrome X", but the constellation of these symptoms is now better known as the metabolic syndrome.

Overnutrition, as seen in obesity, causes ectopic fat accumulation ${ }^{6}$. Ectopic fat accumulation is accumulation of lipid in tissues other than the adipocytes, like for instance liver, muscle and cardiac tissue. These tissues will be challenged to enhance fat oxidation, though recent evidence suggests that oxidative capacity in these tissues is hampered ${ }^{7,8}$. Along with the increase in fatty acid availability, as seen in overnutrition and obesity, fat will be stored, rather than oxidized in these tissues, contributing to excessive ectopic fat accumulation. It is 


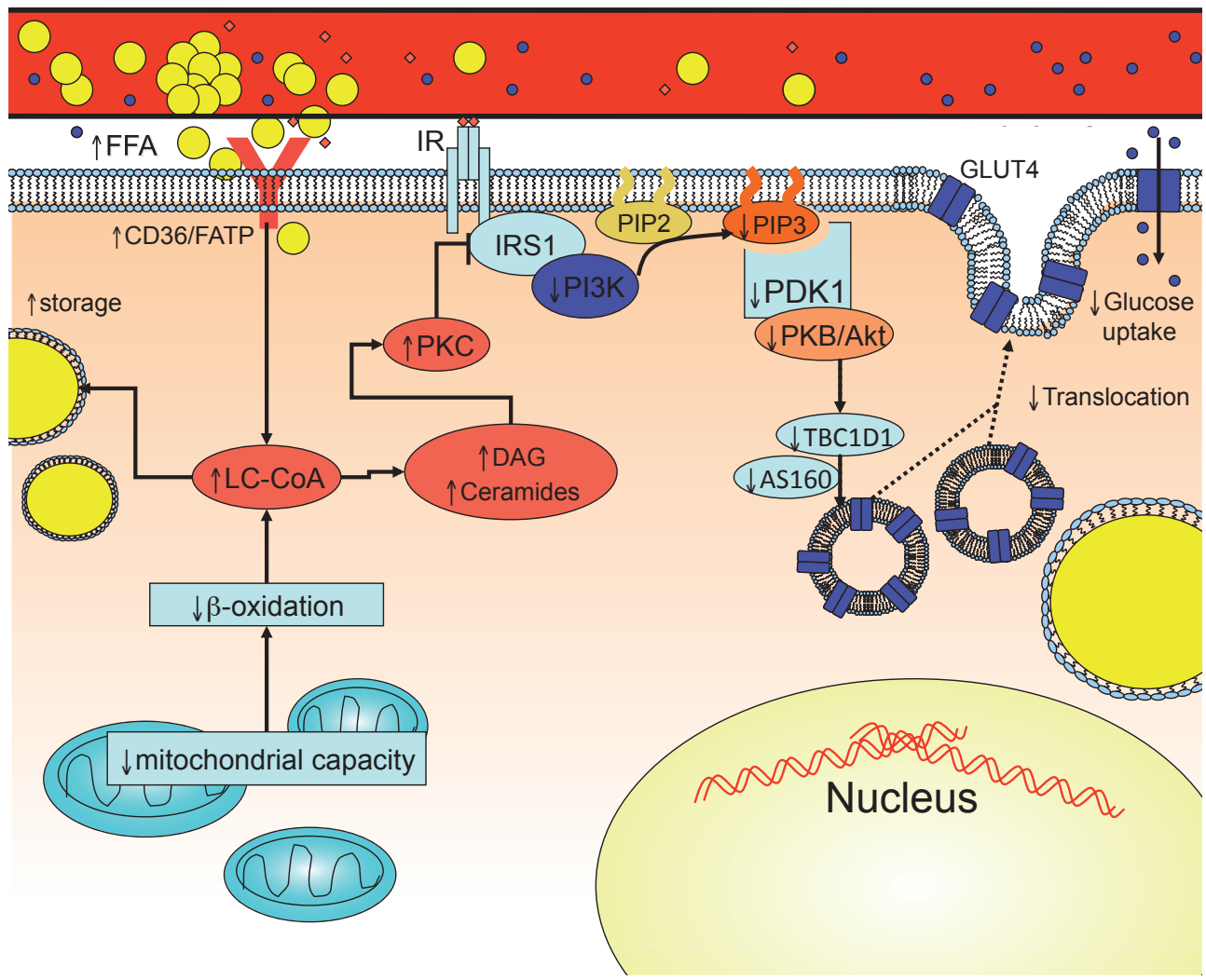

Figure 1: Lipotoxic pathways inducing insulin resistance in skeletal muscle

Insulin resistance due to toxic lipid intermediates; an increased availability of free fatty acids (FFA) in the plasma results in an increased expression of fatty acid transporters (FATP, CD36) and an increased uptake of fatty acids. As mitochondrial function and beta-oxidation are decreased in type 2 diabetes, long-chain fatty acid-CoA (LC-CoA), diacylglycerols and ceramides accumulate in the cytoplasm and lipid droplets. These intermediates interfere with the insulin-signaling cascade via activation of PKC, which directly increases serine phosphorylation of the insulin receptor substrate (IRS). This down-regulates insulin signaling associated with IRS tyrosine phosphorylation by inhibiting PKB in skeletal muscle and results in a reduced uptake of glucose.

the fat accumulation in these tissues that has been related to insulin resistance ${ }^{9-16}$. Not only is ectopic fat accumulation in muscle elevated in type 2 diabetes, but also in the skeletal muscle of first-degree relatives of type 2 diabetic patients, indicating that there might be a predisposed mechanism for the development of type 2 diabetes.

Although there is consensus on the exact involvement of ectopic fat accumulation in the development of insulin resistance, the routes leading to this phenomenon remain an item of discussion. This is mainly caused by the fact that highly insulin sensitive endurance trained 
athletes have an increased fat accumulation in skeletal muscle, while they do not develop insulin resistance. This suggests that lipid accumulation per se is not a bad thing ${ }^{17}$.

It has been suggested that in obesity, rather than in highly trained athletes, there is a mismatch between uptake and oxidation of fatty acids in ectopic tissue. Fat in ectopic tissue is stored in little droplets in the cytoplasm of the cell. These droplets are coated with a phospholipid layer on which regulating proteins are located that control the lipid droplet. It might be that the regulation and dynamics of these proteins and hence the lipid droplets in the cell are changed and that this might lead to a build up of toxic intermediates like for instance fatty acid Acyl-CoA, DAGs and ceramides ${ }^{18}$. These toxic substances are products derived from partial oxidation of fat, hence the name intermediates. These intermediates have been shown to interfere with the insulin signaling cascade via activation of PKC and inhibition of PKB in skeletal muscle and can therefore be responsible for the decreased insulin sensitivity in obesity ${ }^{17,19}$ (see figure 1). Another hypothesis is that that fatty acid intermediates can lead to insulin resistance through the activation of the inflammatory pathway of $I \kappa B / N F \kappa B$, which eventually leads to insulin resistance ${ }^{18,20}$. At last also lipid peroxidation, due to an increased ROS production and abundancy of fatty acids, might damage cellular components, also leading to insulin resistance ${ }^{21}$. Thus, it seems like the balance between fatty acid availability, accumulation and oxidation seems to be detrimental for a normal cellular homeostasis ${ }^{22}$. Nevertheless, as mentioned before, the exact involvement of ectopic fat accumulation in the development of insulin resistance and the routes leading to this phenomenon, remain an item of discussion.

\section{Negative consequences of obesity and type 2 diabetes}

Type 2 diabetes is diagnosed by measuring blood glucose levels. But it is not the glucose levels, but the complications following this hyperglycemia that characterize the disease. As stated by the $\mathrm{WHO}$, diabetes is a condition primarily defined by the level of hyperglycaemia giving rise to the risk of microvascular damage (retinopathy, nephropathy and neuropathy). This microvascular damage is associated with reduced life expectancy, significant morbidity due to specific diabetes related microvascular complications, increased risk of macrovascular complications (ischaemic heart disease, stroke and peripheral vascular disease), and a diminished quality of life. Therefore the cut-off point for the diagnosis of type 2 diabetes was set to the treshold related to an increased risk of developing diabetic retinopathy, which is at a fasting plasma glucose $\geq 7.0 \mathrm{mmol} / \mathrm{l}(126 \mathrm{mg} / \mathrm{dl})$ or $2-\mathrm{h}$ plasma glucose $\geq 11.1 \mathrm{mmol} / \mathrm{l}$ $(200 \mathrm{mg} / \mathrm{dl})^{23}$. 
Indeed it was shown that type 2 diabetic patients are at a significantly greater risk of developing both micro- and macrovascular diseas $\mathrm{e}^{24,25}$. This indicates that cardiovascular diseases are a grave problem in type 2 diabetes and the most common cause of death in this group of patients. Although the heart mostly suffers from ischemic heart disease (heart attacks) and elevated heart pressure in type 2 diabetes, other cardiovascular diseases might also play a part in the morbidity and mortality in type 2 diabetes. In a cohort study done by Rubler et al. in 1972, it was found that type 2 diabetic patients remained at increased risk of heart failure even after correction for vascular disease and hypertension. Since then, this phenomenon has been confirmed by large epidemiological studies ${ }^{26,27}$, which has led to the recognition of a new clinical entity now termed 'diabetic cardiomyopathy'. Although the prevalence of cardiomyopathy without any other cardiovascular co-morbidities is still quite low in type 2 diabetes ${ }^{28,29}$, the co-incidence with micro- and macrovascular disease might aggravate the existing pathology and contribute to an increased mortality and morbidity in type 2 diabetes ${ }^{30,31}$. There are many different theories about the mechanisms responsible for structural changes of the diabetic heart ${ }^{32,33}$. As ectopic lipid accumulation and plasma free fatty acid levels are increased in the obese state and are normalized after life style intervention and reduction in body mass, the negative effects of lipid accumulation have gained more attention in order to explain the etiology of diabetic cardiomyopathy. This thesis will focus on the possible mechanisms, which might be involved in both the development of insulin resistance and the development of diabetic cardiomyopathy. A more detailed overview of the current knowledge on pathological mechanisms putatively contributing to the development of diabetic cardiomyopathy will be given in chapter 2 .

\section{Outline of this Thesis}

As pointed out above, the mechanisms leading to diabetic cardiomyopathy remain to be elucidated. Therefore we reviewed the possible lipotoxic mechanisms that might contribute to this diabetic cardiomyopathy in chapter $\mathbf{2}$.

Subsequently we validated a method to efficiently measure cardiac function in small rodents with MRI in chapter 3 . Then these techniques were applied in an experimental study presented in chapter $\mathbf{4}$, in which we investigated the role of neutral lipid storage in the heart in development of mitochondrial dysfunction and cardiomyopathy. For this purpose we used a mouse model lacking adipose triglyceride lipase (ATGL). ATGL is the main enzyme responsible for triacylglycerol lipolysis. Mice lacking ATGL develop extreme ectopic lipid accumulation (also in the heart) and therefore this serves as a valid model to investigate the role of excessive cardiac lipid accumulation in the heart on the development of cardiomyopathy. As we found that mitochondria indeed play a role herein, we tried to 
extrapolate this to the skeletal muscle as well. As also in skeletal muscle the role of neutral lipid storage in the development of insulin resistance remains unclear, we sought to determine the effect of neutral lipid storage in skeletal muscle on insulin sensitivity and the interplay with skeletal muscle oxidative capacity. This was done again in mice lacking ATGL. The results are presented in chapter 5.

The findings in the animal model showed that the cardiomyopathy in the ATGL-/- model caused a lowered mitochondrial function, via the PPAR-pathways. This changed our idea about the role of lipids in the cellular metabolism as somehow these lipids or fatty acids might also be involved in signaling, but only when available after modification or storage. As these results originate from mice studies, and mice seemed to benefit strikingly from treatment with PPAR agonists, we wondered how these results would translate into humans. Therefore we treated patients with a very rare similar genetic defect in the ATGL gene in chapter 6 with PPAR agonists to assess whether these patients might possibly benefit from this treatment, as did the mice.

To better understand the mechanisms leading to insulin resistance in humans, we did a study in a relatively large group of type 2 diabetic patients and BMI and age matched normoglycemic control subjects. In this study we investigated the different interrelationships of the characteristics of type 2 diabetes. We identified determinants for metabolic flexibility and tried to link insulin sensitivity and mitochondrial function. The results are presented in chapter 7.

Although ectopic lipid storage apparently plays a role in lipotoxicity and insulin resistance, as shown in the previous chapters, the increase in plasma FFA is the main cause of lipid accumulation. Therefore we choose to test the effect of elevated plasma FFA on the heart in a more physiological model. In chapter $\mathbf{8}$ we describe a study in which we elevated plasma FFA with acute exercise in the fasted state and assessed the effect of elevated circulatory fatty acids on cardiac lipid content, energy status and and cardiac function.

In chapter 9 we aimed to reverse the effects of elevated plasma FFA levels by inhibition of peripheral lipolysis with a nicotinic acid analogue (Acipimox). We hoped to decrease cardiac lipid accumulation and improve cardiac function and overall metabolic health by reducing the surplus of the lipids as seen in obesity.

Finally in chapter 10 the findings of the different studies described in this thesis are summarized and discussed in a broader perspective. Also future directions for research within this field of type 2 diabetes and lipid accumulation will be addressed. 


\section{References:}

1. Polonsky KS. The past 200 years in diabetes. N Engl J Med 2012;367:1332-1340.

2. Roglic G, Unwin N, Bennett PH, Mathers C, Tuomilehto J, Nag S, et al. The burden of mortality attributable to diabetes: realistic estimates for the year 2000. Diabetes Care 2005;28:21302135.

3. Mooy JM, Grootenhuis PA, de Vries H, Valkenburg HA, Bouter LM, Kostense PJ, et al. Prevalence and determinants of glucose intolerance in a Dutch caucasian population. The Hoorn Study. Diabetes Care 1995;18:1270-1273.

4. Weyer C, Bogardus C, Mott DM, Pratley RE. The natural history of insulin secretory dysfunction and insulin resistance in the pathogenesis of type 2 diabetes mellitus. J Clin Invest 1999;104:787-794.

5. Kahn SE, Hull RL, Utzschneider KM. Mechanisms linking obesity to insulin resistance and type 2 diabetes. Nature 2006;444:840-846.

6. Frayn KN. Adipose tissue as a buffer for daily lipid flux. Diabetologia 2002;45:1201-1210.

7. Saltiel AR. New perspectives into the molecular pathogenesis and treatment of type 2 diabetes. Cell 2001;104:517-529.

8. McGarry JD. What if Minkowski had been ageusic? An alternative angle on diabetes. Science 1992;258:766-770.

9. Unger RH. Lipotoxicity in the pathogenesis of obesity-dependent NIDDM. Genetic and clinical implications. Diabetes 1995;44:863-870.

10. Boden G. Obesity, insulin resistance and free fatty acids. Curr Opin Endocrinol Diabetes Obes 2011;18:139-143.

11. Boden G, Lebed B, Schatz M, Homko C, Lemieux S. Effects of acute changes of plasma free fatty acids on intramyocellular fat content and insulin resistance in healthy subjects. Diabetes 2001;50:1612-1617.

12. Badin PM, Louche K, Mairal A, Liebisch G, Schmitz G, Rustan AC, et al. Altered skeletal muscle lipase expression and activity contribute to insulin resistance in humans. Diabetes 2011;60:1734-1742.

13. Felber JP, Ferrannini E, Golay A, Meyer HU, Theibaud D, Curchod B, et al. Role of lipid oxidation in pathogenesis of insulin resistance of obesity and type II diabetes. Diabetes 1987;36:13411350.

14. Krssak M, Roden M. The role of lipid accumulation in liver and muscle for insulin resistance and type 2 diabetes mellitus in humans. Rev Endocr Metab Disord 2004;5:127-134.

15. Perseghin G, Scifo P, De Cobelli F, Pagliato E, Battezzati A, Arcelloni C, et al. Intramyocellular triglyceride content is a determinant of in vivo insulin resistance in humans: a $1 \mathrm{H}-13 \mathrm{C}$ nuclear magnetic resonance spectroscopy assessment in offspring of type 2 diabetic parents. Diabetes 1999;48:1600-1606.

16. Befroy DE, Petersen KF, Dufour S, Mason GF, de Graaf RA, Rothman DL, et al. Impaired mitochondrial substrate oxidation in muscle of insulin-resistant offspring of type 2 diabetic patients. Diabetes 2007;56:1376-1381.

17. Goodpaster BH, He J, Watkins S, Kelley DE. Skeletal muscle lipid content and insulin resistance: evidence for a paradox in endurance-trained athletes. J Clin Endocrinol Metab 2001;86:5755-5761. 
18. Itani SI, Ruderman NB, Schmieder F, Boden G. Lipid-induced insulin resistance in human muscle is associated with changes in diacylglycerol, protein kinase C, and IkappaB-alpha. Diabetes 2002;51:2005-2011.

19. Shulman GI. Cellular mechanisms of insulin resistance. J Clin Invest 2000;106:171-176.

20. Sriwijitkamol A, Christ-Roberts C, Berria R, Eagan P, Pratipanawatr T, DeFronzo RA, et al. Reduced skeletal muscle inhibitor of kappaB beta content is associated with insulin resistance in subjects with type 2 diabetes: reversal by exercise training. Diabetes 2006;55:760-767.

21. Schrauwen $\mathrm{P}$, Hesselink MK. Oxidative capacity, lipotoxicity, and mitochondrial damage in type 2 diabetes. Diabetes 2004;53:1412-1417.

22. Moro C, Bajpeyi S, Smith SR. Determinants of intramyocellular triglyceride turnover: implications for insulin sensitivity. Am J Physiol Endocrinol Metab 2008;294:E203-213.

23. WHO I. Definition and diagnosis of diabetes mellitus and intermediate hyperglycemia. 2006:50.

24. Garcia MJ, McNamara PM, Gordon T, Kannel WB. Morbidity and mortality in diabetics in the Framingham population. Sixteen year follow-up study. Diabetes 1974;23:105-111.

25. Haffner SM, Lehto S, Ronnemaa T, Pyorala K, Laakso M. Mortality from coronary heart disease in subjects with type 2 diabetes and in nondiabetic subjects with and without prior myocardial infarction. N Engl J Med 1998;339:229-234.

26. Rubler S, Dlugash J, Yuceoglu YZ, Kumral T, Branwood AW, Grishman A. New type of cardiomyopathy associated with diabetic glomerulosclerosis. Am J Cardiol 1972;30:595-602.

27. Kannel WB, Hjortland M, Castelli WP. Role of diabetes in congestive heart failure: the Framingham study. Am J Cardiol 1974;34:29-34.

28. Poirier P, Bogaty P, Garneau C, Marois L, Dumesnil JG. Diastolic dysfunction in normotensive men with well-controlled type 2 diabetes: importance of maneuvers in echocardiographic screening for preclinical diabetic cardiomyopathy. Diabetes Care 2001;24:5-10.

29. Zabalgoitia M, Ismaeil MF, Anderson L, Maklady FA. Prevalence of diastolic dysfunction in normotensive, asymptomatic patients with well-controlled type 2 diabetes mellitus. Am J Cardiol 2001;87:320-323.

30. McGavock JM, Lingvay I, Zib I, Tillery T, Salas N, Unger R, et al. Cardiac steatosis in diabetes mellitus: a 1H-magnetic resonance spectroscopy study. Circulation 2007;116:1170-1175.

31. McGavock JM, Victor RG, Unger RH, Szczepaniak LS. Adiposity of the heart, revisited. Ann Intern Med 2006;144:517-524.

32. Spector KS. Diabetic cardiomyopathy. Clin Cardiol 1998;21:885-887.

33. Boudina S, Abel ED. Diabetic cardiomyopathy revisited. Circulation 2007;115:3213-3223. 

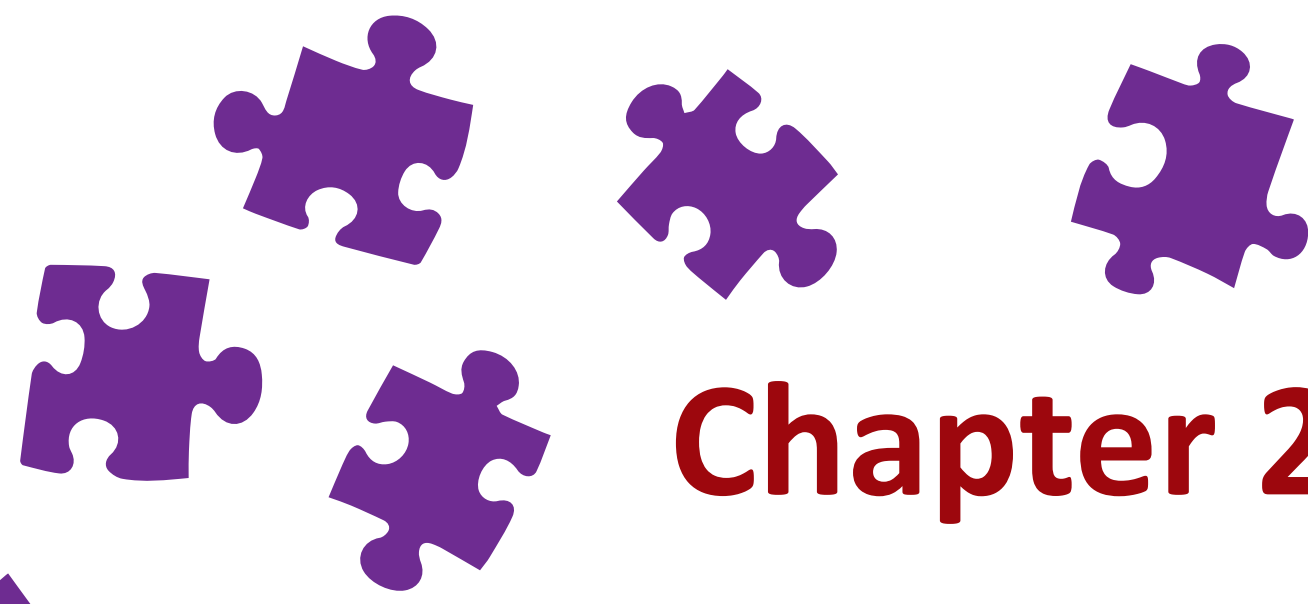

Chapter 2

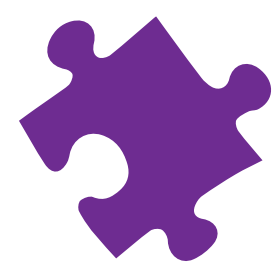

\section{Review:}

\section{Lipotoxicity in type 2 diabetic cardiomyopathy}

\section{Tineke van de Weijer ${ }^{1}$, Vera B. Schrauwen-Hinderling ${ }^{2}$, and Patrick Schrauwen ${ }^{1}$}

${ }^{1}$ Department of Human Biology, Maastricht University Medical Centre, Maastricht, The Netherlands; and ${ }^{2}$ Department of Radiology, School of Nutrition and Metabolism, NUTRIM, Maastricht University Medical Centre,

The Netherlands 


\section{Abstract}

As obesity and type 2 diabetes are becoming an epidemic in westernized countries, the incidence and prevalence of obesity- and diabetes-related co-morbidities are increasing. In type 2 diabetes ectopic lipid accumulation in the heart has been associated with cardiac dysfunction and apoptosis, a process termed lipotoxicity. Since cardiovascular diseases are the main cause of death in diabetic patients, diagnosis and treatment become increasingly important. Although ischaemic heart disease is a major problem in diabetes, non-ischaemic heart disease (better known as diabetic cardiomyopathy) becomes increasingly important with respect to the impairment of cardiac function and mortality in type 2 diabetes. The underlying aetiology of diabetic cardiomyopathy is incompletely understood but is beginning to be elucidated. Various mechanisms have been proposed that may lead to lipotoxicity. Therefore, this review will focus on the mechanisms of cardiac lipid accumulation and its relation to the development of cardiomyopathy. 


\section{Introduction}

Type 2 diabetic patients are at a significantly greater risk of developing both micro- and macrovascular disease ${ }^{1,2}$. Even after adjusting for concomitant risk, diabetic individuals remained at increased risk of heart failure. This phenomenon was first described by Rubler et al. in 1972 in diabetic patients with heart failure but without hypertension or coronary artery disease. Since then this phenomenon has been confirmed by large epidemiological studies $^{3,4}$. This has led to the recognition of a new clinical entity now termed 'diabetic cardiomyopathy'. This disease is defined by structural changes in the heart, such as increased LV mass, fibrosis and a dilation of the ventricles ${ }^{5}$ in the absence of ischemia or alterations in blood pressure ${ }^{3,6}$. Diabetic cardiomyopathy is mainly characterized by diastolic dysfunction, which may precede the development of systolic dysfunction ${ }^{7,8}$. Although the prevalence of this disease without any other cardiovascular co-morbidities is still quite rare in type 2 diabetes $^{9,10}$, the co-incidence with micro- and macrovascular disease, might aggravate the existing pathology and lead to an increased mortality and morbidity in type 2 diabetes ${ }^{11,12}$. Therefore, a better understanding and treatment of this disease is needed.

It has been well established that indeed the greater incidence of physical inactivity, hypertension and hyperlipidemia in obese patients are main contributors to the development of cardiac disease in obesity and type 2 diabetes ${ }^{13,14}$. Furthermore, the reversal of these effects by reducing body mass and introducing a more physically active lifestyle, appear to significantly reduce the risk of cardiovascular diseases ${ }^{15,16}$. Nonetheless, the underlying mechanisms are incompletely understood. There are many different theories about the mechanisms leading to structural changes of the diabetic heart ${ }^{17}{ }^{18}$. As lipid accumulation and plasma free fatty acid levels are increased in the obese state and are normalised after life style intervention and reduction in body mass, the negative effects of lipid accumulation have gained more attention in order to explain the aetiology of diabetic cardiomyopathy. Therefore, in this review we will focus on lipotoxic mechanisms possibly leading to diabetic cardiomyopathy in obesity and type 2 diabetes. Roughly these mechanisms can be divided into two main categories 1 ) lipotoxic mechanisms that impair cardiac energy metabolism and 2) mechanisms that lead to lipoapoptosis.

Regarding cardiac energy metabolism, $\mathrm{O}_{2}$ consumption of the heart has been shown to be increased and ATP/O ratios to be lower in type 2 diabetic hearts and in hearts under fatty acid infusion, indicating a decrease in cardiac efficiency in the prediabetic as well as in the diabetic state ${ }^{19-21}$. Possible underlying mechanisms will be discussed in more detail in paragraph 3.1. 
At the basis of cardiac lipotoxicity is the excessive accumulation of fat or fatty acid intermediates in cardiomyocytes. Therefore, understanding cardiac lipid uptake and mechanisms involved in the upregulation of cardiac lipid uptake is needed in order to understand the development of cardiac lipid accumulation and ultimately cardiac lipotoxicity. 


\section{Why does fat accumulate in cardiac muscle?}

\subsection{Role of fatty acid uptake and transporters}

Chronic overnutrition is associated with increased plasma concentrations of free fatty acids $^{22,23}$, which are probably due to an increased adipose tissue insulin resistance ${ }^{24,25}$. A chronic nutrient overload leads to an increase in adipose tissue, and if the storage capacity and expandability of these adipocytes is low, the adipocytes will become hypertrophic. This may, in turn, create local hypoxia and lead to release of pro-inflammatory factors. The latter have been shown to be able to suppress insulin signaling. In insulin-resistant adipocytes, lipolysis is incompletely suppressed which causes an increased release of free fatty acids (for a more detailed overview see review ${ }^{25}$ ) leading to cardiac lipid accumulation ${ }^{26-28}$.

The increase in plasma free fatty acids has been associated with an increased cardiac fatty acid uptake. For instance, in ob/ob mice (leptin deficient mice), obesity increased plasma fatty acid availability and resulted in an increased cardiac lipid content ${ }^{29}$. This mechanism is not pathological per se, as under physiological conditions such as, fasting or exercise, myocardial lipid content can be elevated significantly ${ }^{30,31}$. This illustrates that elevated circulating free fatty acid levels augment cellular uptake and stimulate storage of triglycerides. However, this raises the question of how free fatty acid uptake is regulated in cardiac muscle and if this regulation is altered in obesity and type 2 diabetes.

Uptake of plasma free fatty acids can occur through passive diffusion via a so-called flipflop mechanism ${ }^{32}$. Transbilayer flip-flop is the process by which lipids are moved between the two leaflets of the membrane bilayer spontaneously ${ }^{33}$. A protein-mediated process facilitates this fatty acid transport. For cardiac tissue there are three recognized groups of fatty acid transporting proteins; CD36 (human homologue of Fatty Acid Transporter protein FAT), FABP-pm (plasma membrane fraction of Fatty Acid Binding Protein) and FATP1,4 and 6

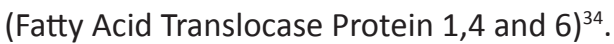

In the heart FAT/CD36 and FABPpm appear to be key transporters involved in fatty acid uptake $^{35}$. CD36 was shown to be responsible for up to $60 \%$ of the fatty acid uptake in the heart ${ }^{36}$. Overexpression of CD36 in cardiac muscle increased the rate of fatty acid uptake and increased fatty acid metabolism, whereas knockdown of CD36/FAT knocked-down reduced fatty acid uptake and metabolism ${ }^{37,38}$. Similar to GLUT4, CD36 is present in cellular vesicles and can be translocated to the cell membrane rapidly upon acute stimulation by insulin, muscle contraction or AMP-kinase activation. This ability of CD36 to be able to translocate to the plasma membrane and thereby stimulate FA uptake under certain conditions is specific for CD36. The acute regulation involves the translocation of CD36 to the plasma membrane stimulated by insulin, muscle contraction and AMP-kinase activation. Furthermore, CD36 
can traffic between the endosomes and the nuclear membrane, whereas the other fatty acid transport proteins do not have this capability ${ }^{39}$. Longer term regulation of CD36 involves ubiquitination. It was shown that insulin attenuates ubiquitination, thereby increasing the availability of CD36 for translocation and uptake of FFA. In contrast, FFA enhance ubiquination, thereby increasing CD36 degradation and creating a negative feedback ${ }^{40}$.

Although total concentration of CD36 was unchanged in obesity and diabetes, permanent translocation of CD36 to the plasma membrane seemed to occur ${ }^{41-43}$. It is suggested that the resulting increased uptake of fatty acids may lead to substrate competition with glucose, and therefore CD36 may also play an important role in the development of lipid-induced insulin resistance ${ }^{41,42}$.

Cardiac-specific FATP-1 overexpression has also been shown to increase fatty acid uptake by 4 -fold and cardiac lipid accumulation by 2 -fold ${ }^{44}$. It has been speculated, from studies in skeletal muscle that FATP-1-facilitated fatty acid uptake mainly serves to fuel oxidation, though evidence concerning the heart is still lacking. Also, very little is known about the physiological stimuli that may regulate the expression of FATP-1 ${ }^{45}$. FATP-4 is expressed in cardiac tissue, but the contribution to lipid uptake in cardiac tissue is still undetermined ${ }^{34,46}$. FATP-6 is only expressed in the heart and has been shown to enhance free fatty acid transport in cultured cardiomyocytes ${ }^{47}$. FATP-6 only promoted fatty acid uptake slightly when expressed in yeast, and its role in vivo is still under debate $e^{48,49}$.

\subsection{Fatty acid storage}

Facilitated uptake of circulating fatty acids is an important regulatory step that affects overall cardiac fat content. However, next to fatty acid uptake, triglyceride storage and lipolysis inside cardiomyocytes will ultimately determine the fate of the fatty acids.

One of the genes that has been intensively studied in the context of cardiolipotoxicity is GPAT. Glycerol-3-phosphate acyltransferase (GPAT) is the rate-limiting step in TG synthesis, which can be inhibited by ACC-stimulated AMPK activation via malonyl-CoA. In some ${ }^{50,51}$, although not all ${ }^{52}$ rodent models of diabetes, GPAT activity was found to be enhanced in cardiac tissue. Interestingly, deletion of GPAT1 protected mice from the effects of a high fat diet on cardiac dysfunction and fat accumulation ${ }^{51}$. So far, it remains unclear whether GPAT activity is increased in human diabetic cardiomyopathy. Another gene that has received considerable interest is DGAT. Thus, increased DGAT activity has been linked to lipid accumulation and cardiomyopathy. Glenn et al. ${ }^{53}$ recently found increased lipid accumulation in a cardiomyocyte-selective DGAT1 transgenic mouse model. On the other 
hand, when DGAT1 overexpression was crossed with a model of lipotoxic cardiomyopathy, it seemed to protect against the development of cardiac dysfunction ${ }^{54}$. Therefore, it seems that DGAT might play a dual role in the development of lipotoxic cardiomyopathy.

Ueno et al. ${ }^{55}$ investigated the role of HSL, which is involved in lipolysis of TG, in cardiac lipotoxicity in diabetic mice. In this study heart-specific HSL overexpressing (MHC-HSL) mice were used and diabetes was induced by administration of streptozotocin. Diabetic MHC-HSL mice had no lipid droplet formation in the heart upon a high fat diet, unlike the wild type animals, thus demonstrating that the regulation of lipolysis is an important determinant of cardiac lipid content. Also, ATGL deficiency has been associated with a severe form of cardiac lipid accumulation and the development of cardiomyopathy both in mice ${ }^{56,57}$ and in humans ${ }^{58}$. Therefore a decent function of ATGL seems crucial for maintaining a normal cardiac function.

Taken together, a dysbalance between fatty acid uptake, TG synthesis and lipolysis may result in net fat storage in the heart, ultimately leading to cardiac fat accumulation. In chapter 3.1.1 we will also discuss how alterations in fatty acid and glucose oxidation may affect cardiac function and induce insulin resistance of the heart. 


\section{The association between cardiac steatosis and cardiomyopathy in type 2 diabetes.}

\subsection{Impaired cardiac energetics}

Low cardiac ATP production is associated with a decreased contractility in the absence

of ischemia ${ }^{19,59}$. For cardiomyocyte contraction, interactions between ATP and calcium are required in appropriate amounts ${ }^{60}$. Therefore, a decrease in ATP or impaired calcium handling, could drastically impair cardiac contractility.

\subsubsection{Altered substrate metabolism}

The notion that type 2 diabetes leads to altered regulation of cardiac metabolism is well established. ${ }^{61,62}$ In the prediabetic and diabetic state, myocardial $\mathrm{O}_{2}$ consumption and fatty acid oxidation are increased and cardiac efficiency is. ${ }^{19,21}$ In type 2 diabetic patients a 2-fold increase in cardiac palmitate oxidation and a 30-40\% decrease in glucose oxidation has been shown. ${ }^{20,21} \mathrm{In} d b / d b$ mice (mice with a defect in the leptin receptor) alterations in substrate metabolism were paralleled by a decreased contractility eventually leading to cardiomyopathy. ${ }^{61,63}$ Other studies, performed with isolated working hearts of diabetic animals, confirmed that a high rate of fatty acid oxidation is associated with ventricular dysfunction. ${ }^{59,64}$ These results strongly suggest a causative role for altered substrate metabolism in the development of cardiomyopathy in $\mathrm{db} / \mathrm{db}$ diabetic hearts.

For a long time, it was thought that the Randle cycle was the main contributor to the biochemical shift towards fatty acid uptake and oxidation in type 2 diabetes. Randle proposed that a higher rate of release of fatty acids and ketone bodies for oxidation was responsible for the decrease in glucose oxidation and uptake ${ }^{65}$. Though substrate competition plays an important role in type 2 diabetes, increasing evidence suggests that there are also some cellular maladaptations that are important in the regulation of substrate oxidation. The gene regulatory pathway of PPAR- $\alpha$ was identified as an important determinant of the shift in substrate metabolism in type 2 diabetes. In mice with cardiac-restricted overexpression of PPAR $\alpha$ (MHC-PPAR), it was found that PPAR- $\alpha$ is involved in the upregulation of CPT1 in mitochondria, which increases the uptake of LCFA into mitochondria and facilitates the fatty acids to undergo beta-oxidation ${ }^{66}$. In the same mouse model, the increase in fatty acid oxidation was paralleled by decreased left ventricular function on a high-fat diet. Paradoxically, chronic exposure to elevated free fatty acids seems to down-regulate PPAR- $\alpha$ in rodent cardiomyocytes. This down-regulation has been proposed to further decrease cardiac function by inhibition of fatty acid oxidation and increased intracellular fat 
accumulation $^{67,68}$. Human studies on PPAR expression in the heart are scarce. Marfella et al. ${ }^{69}$ showed an increased expression of SREBP1c and PPAR- $\gamma$ in patients with the metabolic syndrome, which was associated with an increased fat accumulation in the heart and poor cardiac function. Although PPAR- $\alpha$ was not changed in these subjects, the increased expression of SREPB1C and PPAR- $\gamma$ might indicate that there are some aberrations in PPARregulated pathways, which lead to an increased fat uptake and storage in the metabolic syndrome. Conversely, other studies such as that of Anderson et al. ${ }^{21}$, did not detect a change in PPAR- $\alpha$ expression in the hearts of type 2 diabetes patients. Therefore, it remains unclear whether these mechanisms found in animal studies can be fully translated from animal studies to the humans condition.

Although we focus on the negative effects of lipid accumulation in this review, it is important to note that the fate of glucose and fatty acids are intertwined. Besides inducing lipid accumulation and stimulating fat oxidation, PPAR- $\alpha$ activation can also decrease glucose utilization by increasing the expression of pyruvate dehydrogenase kinase $4(\text { PDK- } 4)^{70}$. It is hypothesized that this mechanism protects against glucose overload in addition to the already present lipid overload and thus making the heart insulin resistant ${ }^{65,71}$. This again contributes to a more rigorous shift in substrate metabolism towards fatty acid oxidation. In a chronic state this lipid induced PDK-4 activity leads to increased intracellular glucose concentration, resulting in glucotoxicity. Chronically high glucose concentration increases the flux through the hexosamine biosynthethic pathway and increases $\mathrm{N}$-acetyl-glucosamine production. This has been associated with insulin resistance, ROS production, hypertrophy and apoptosis in perfused hearts and cell studies, thus augmenting the lipotoxic effects ${ }^{72-74}$. So it seems that the PPAR $\alpha$-induced increase in fat utilization in the diabetic heart may initially serve as a compensatory mechanism to adjust substrate oxidation to supply, though chronic derangements in cardiac metabolism may have maladaptive consequences, including glucotoxicity and functional cardiac abnormalities. However, the role of these metabolic changes in the development of cardiac dysfunction is poorly understood.

The shift in substrate oxidation itself is often hypothesized as one of the main factors contributing to the development of contractile dysfunction in diabetes ${ }^{75,76}$. It is suggested that reliance on fatty acid oxidation will reduce cardiac energy efficiency, and thereby contributes to cardiomyopathy. Theoretically, more oxygen is required for the production of ATP from fatty acids compared with glucose ${ }^{77}$. Thus, when shifting from $100 \%$ palmitate oxidation to $100 \%$ glucose oxidation, a $12 \%$ decrease in the amount of oxygen required for ATP synthesis would occur ${ }^{77}$. However, a total shift from fat to glucose oxidation will never occur under physiological conditions. Therefore, while increased concentrations of exogenous 
fatty acids resulted in a marked increase in basal myocardial oxygen requirements ${ }^{78,79}$, this increase in oxygen requirement and accompanying decrease in ATP-production is too large to be explained solely by a shift from glucose to fat oxidation ${ }^{78}$.

\subsubsection{Mitochondrial uncoupling, dysfunction and ROS production}

Uncoupling describes the lowering of the proton gradient over the inner mitochondrial membrane without production of ATP. In this way, uncoupling lowers the production of mitochondrial ATP, thereby making the heart less efficient, which could contribute to the development of cardiac contractile dysfunction in type 2 diabetes.

In isolated hearts of ob/ob mice, perfusion with FFA compared to glucose led to increased oxygen consumption and a reduced ATP/O ratio. These changes in ATP/O ratio were too large to be accounted for by changes in substrate metabolism, which was interpreted as increased mitochondrial uncoupling ${ }^{75,80}$. Indeed, the increase in fatty acid metabolism in these hearts was associated with an increased expression of mitochondrial uncoupling proteins ${ }^{81}$. Similar results were found for $d b / d b$ mice $^{63}$. It is known that fatty acids can induce uncoupling via PPAR- $\alpha$-induced upregulation of UCP3 ${ }^{67}$. Also in humans, the expression of UCP3 was related to circulating plasma fatty acid levels ${ }^{82}$. In addition to an effect of FA on the induction of uncoupling proteins, fatty acids themselves may also activate the process of mitochondrial uncoupling themselves ${ }^{83,84}$. This uncoupling effect is due to a cyclic movement of undissociated fatty acids with the release of protons into the matrix ${ }^{85,86}$.

Though uncoupling might be detrimental for cardiac efficiency, a protective function has also been described. It is hypothesized that mitochondrial uncoupling serves to limit the production of reactive oxygen species by the mitochondria, as it has been shown that membrane potential displays a negative relationship with mitochondrial ROS production ${ }^{87}$, ${ }^{88}$. This suggests that a very mild lowering of the proton gradient may already markedly lower the production of $\operatorname{ROS}^{20,89}$. ROS can damage mitochondria and other cellular components by oxidizing proteins, converting lipids into reactive lipid peroxides, increase protein tyrosine nitration and damage $D N A^{90,91}$. If extensive damage occurs, this can lead to mitochondrial dysfunction, altered cellular function or even apoptosis. Mitochondrial ROS production has been shown to impair contractility of cardiomyocytes in vitro ${ }^{92}$. Boudina et al. ${ }^{93}$ showed an increased mitochondrial $\mathrm{H}_{2} \mathrm{O}_{2}$ production in cardiomyocytes of ob/ob mice. This was not only paralleled by increased cardiac lipid accumulation and mitochondrial dysfunction, but also by an increase of ROS scavengers in the heart ${ }^{63}$. Also in $d b / d b$ mice an increased ROS production was found, which was associated with an increased apoptosis of cardiomyocytes ${ }^{68,94}$. The study of Anderson et al. ${ }^{21}$ is the only study in humans indicating 
an increase in cardiac ROS production. They found that diabetic patients have an increased mitochondrial $\mathrm{H}_{2} \mathrm{O}_{2}$ emission during oxidation of lipid-based substrates compared to carbohydrate based substrates and depleted glutathione, which are evidence of persistent oxidative stress in atrial tissue ${ }^{21}$.

As mentioned above, ROS can have direct negative effects on mitochondria, thereby impairing cardiac energetics. If ROS concentration is indeed elevated in the diabetic heart, these patients are expected to be at increased risk for mitochondrial dysfunction. Indeed, it has been shown that the intrinsic function of cardiac mitochondria in type 2 diabetes is decreased. In mouse models of type 2 diabetes and obesity, myocardial mitochondrial function and ATP synthesis rates were impaired which was associated with cardiac dysfunction and lipid accumulation ${ }^{95-97}$. Boudina et al. ${ }^{63}$ showed a decreased intrinsic mitochondrial function in $\mathrm{db} / \mathrm{db}$ mice, measured in permeabilised cardiac fibers. This was paralleled by a decreased ATP-production, lower ATP-ase expression and a 2.2 fold increase in cardiac lipid accumulation. Palmitate infusion in these animals further deteriorated mitochondrial function, as uncoupled respiration increased and ATP/O ratios decreased, thus indicating induction of mitochondrial uncoupling in addition to the already existing deficit in mitochondrial respiration. ${ }^{63} \mathrm{How}$ et al. ${ }^{97}$ showed that cardiac efficiency, expressed as the ratio of cardiac work (pressure-volume area (PVA)) over myocardial oxygen consumption $\left(\mathrm{MVO}_{2}\right)$, decreased as $\mathrm{MVO}_{2}$ increased by around 86 and $57 \%$ in $d b / d b$ and STZ-administered hearts, respectively. In another study ${ }^{98}$ they showed that perfusing isolated working hearts with a higher concentration of fatty acids did not change the performance or contractile efficiency, though it did increase oxygen consumption, thus indicating an FA-induced increase in the unloaded $\mathrm{MVO}_{2}$. This effect was more pronounced in the hearts of $d b / d b$ mice. These data suggest that increased in fatty acid availability makes the heart less efficient.

In human studies direct evidence of decreased cardiac mitochondrial function in type 2 diabetes comes from the study of Anderson et al. ${ }^{21}$ They measured ex vivo mitochondrial respiratory capacity in permeabilized myofibers derived from atrial tissue of type 2 diabetic patients and found lower rates of respiration in diabetic patients. These findings were paralleled by increased cardiac lipid accumulation, indicating that lipid accumulation may be associated with impaired mitochondrial function. Furthermore, impaired intrinsic mitochondrial function of the diabetic heart is supported by studies using ${ }^{31} \mathrm{P}$ magnetic resonance spectroscopy (MRS) in humans. MRS can be used to determine energy status of the heart in vivo non-invasively, by determining the Phosphocreatine/ATP ratio (PCr/ATP ratio). MRS experiments demonstrated that type 2 diabetic patients had reduced PCr/ATP ratios, suggesting that mitochondrial high energy phosphate metabolism may be impaired; however changes in the creatine pool and increased ATP utilization, as opposed to ATP 
production, may have also contributed to this observation ${ }^{99-101}$. Furthermore, it was shown that in diabetic patients with normal morphology of the heart, PCr/ATP correlates negatively with serum $\mathrm{FFA}^{99}$, indicating that an increase in FFA availability might impair cardiac energy reserves. On the contrary, studies with well-controlled type 2 diabetic subjects do not show any changes in the PCr/ATP ratio ${ }^{20,102}$. Difference in these findings may be due to differences in co-morbidities of the study populations such as low glycemic control, high blood pressure and dyslipedemia.

Another lipotoxic mechanism linking mitochondrial dysfunction with cardiac structural changes is impaired calcium handling. Upon electrical stimulation, calcium is released from the sarcoplasmic reticulum in order for calcium to bind troponin $\mathrm{C}$ on the actin filaments. This leads to changes in the contractile regulatory proteins allowing interaction between actin and myosin cross-bridges. ATP is required for initiating a new contractile cycle and for the re-uptake of calcium in the sarcoplasmic reticulum via Calcium-ATPase 2a (SERCA2a). Besides the insulin-induced changes in calcium fluxes, a decrease in ATP production also leads to an impaired mitochondrial calcium handling, which contributes to a decreased cardiac contraction ${ }^{60,103}$

In animal studies, calcium handling was altered in the type 2 diabetic $(d b / d b)$ state ${ }^{59,104}$. Also, ob/ob mice had a slowed intracellular decay of calcium and impaired mitochondrial calcium handling upon electrical stimulation, which was associated with increased intramyocellular lipid accumulation in these cells ${ }^{105,106}$. Upon high fat feeding, it was shown that impaired calcium handling due to a lower cardiac ATP supply was the main cause of cardiac dysfunction in the absence of apoptosis ${ }^{107}$. Thus, while there is evidence that calcium handling is impaired in type 2 diabetes and mitochondria have been shown to play a part in impaired calcium handling in obesity models, it has not been directly investigated whether the impaired $\mathrm{Ca} 2+$ handling in diabetes is also caused by mitochondrial impairments.

Together, these data suggest that increased fat uptake in the heart could lead to reduced energy efficiency by inducing mitochondrial damage and uncoupling, increasing ROS production and via impairment of mitochondrial calcium handling. These studies highlight the importance of proper mitochondrial function in type 2 diabetes in order to maintain a normal cellular homeostasis. 
Figure 1: Mechanisms involved in decreasing cardiac energetics.

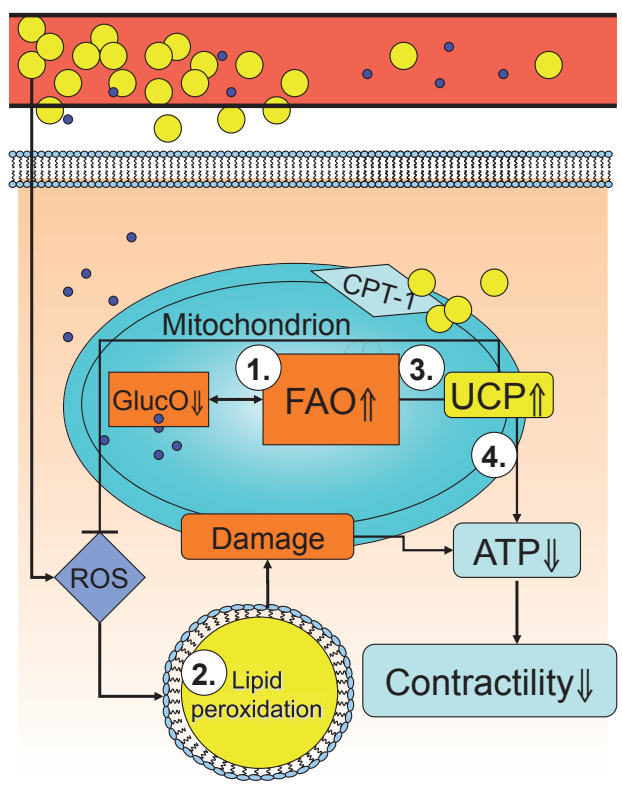

(1.) Change in substrate supply increases fatty acid oxidation, decreases glucose oxidation and reduces ATP/O ratios. Furthermore, increased fatty acid oxidation is associated with an increased ROS production. ROS oxidizes lipids stored in the cytoplasm into lipid peroxides (2.), inducing cellular damage, as well as mitochondrial damage, further decreasing mitochondrial function. The presence of fatty acids also stimulates uncoupling (3.), further decreasing energy production and contractility (4.). On the other hand, uncoupling may also be cardioprotective by decreasing ROS production.

\subsection{Lipoapoptosis}

Besides changes in cardiac efficiency, lipoapoptosis may also contribute to the decreased function of the fatty heart. Lipoapoptosis can be based on different mechanisms like palmitate toxicity, ceramide and DAG formation, ER-stress, membrane destabilization and inflammation.

\subsubsection{Palmitate toxicity}

Palmitate (a saturated fatty acid) can induce apoptosis in isolated cardiomyocytes, independent of ceramide formation ${ }^{108,109}$. Although this toxicity of fatty acid incubation in vitro has been implicated in the pathophysiology of cardiovascular disease, the effects on cardiac myocytes remain incompletely understood.

Several studies investigated the mechanisms through which palmitate may induce apoptosis. It was shown that palmitate induced-activation of the stress-associated protein kinases induced apoptosis in $20 \%$ of the cardiomyocytes ${ }^{110}$. Strikingly, it was found that the effects on apoptosis through palmitate exposure in cardiomyocytes were inhibited by titrating low concentrations of oleate ${ }^{110}$. Furthermore, increasing mitochondrial uptake of palmitate with L-carnitine decreased apoptosis while decreasing uptake with the carnitine palmitoyl 
transferase-1 inhibitor perhexiline almost doubled palmitate-induced apoptosis ${ }^{110}$. This indicates that cytosolic accumulation of saturated fatty acids is detrimental for cellular function.

\subsubsection{Ceramide formation}

Ceramide-induced apoptosis is thought to be an important contributor to the development of lipotoxic cardiomyopathy. This concept was based on several in vitro and in vivo studies. When fatty acid oxidation was reduced in vitro, intracellular triglycerides and ceramides were increased and induced caspase-3 activity and DNA-laddering ${ }^{111,112}$. In various rodent models of lipotoxic cardiomyopathy, diabetes and obesity, increased myocardial ceramide content has been observed in association with cardiac dysfunction ${ }^{66,68}$. For instance, in the study of Park et al. ${ }^{113}$ the lipotoxic dilated cardiomyopathy of the LPL ${ }^{\text {GPI }}$ mice was rescued by pharmacological inhibition of ceramide synthesis via myriocin. This therapy restored ceramide levels to WT levels and normalized substrate utilization of the heart. Both changes were associated with the improvement of cardiac function ${ }^{113}$. Crossing the LPL ${ }^{\mathrm{GPI}}$ mice with $\mathrm{LCB}^{+/-}$mice that have a deletion in the serine palmitoyltransferase enzyme (SPT; the enzyme that initiates the conversion of fatty-acyl co-A into ceramides) gave similar results to that of the administration of myriocin alone ${ }^{68,113}$. Thus, these data suggest that ceramides play an important role in the development of lipotoxic cardiomyopathy.

There is only one human study that assessed the role of ceramides in lipoapoptosis in the human heart. Baranowski et al. ${ }^{114}$ examined the relationship between myocardial apoptosis and ceramide levels in obese and type 2 diabetic patients. Compared with lean subjects, the markers for apoptosis were higher in the myocardium of obese patients and increased further in type 2 diabetic subjects. However, ceramide and sphingoid base content were similar in both groups. Furthermore, mRNA levels of enzymes involved in synthesis and degradation of ceramides were markedly increased in both obese and type 2 diabetic subjects. These results challenge the view that ceramides are the main determinant of apoptosis in the fatty heart of obese and diabetic humans.

Besides inducing apoptosis, ceramides can acutely inhibit insulin-stimulated glucose uptake, GLUT4 translocation and glycogen synthesis. ${ }^{113}$ These effects appear to result from the ability of sphingolipid to block activation of either IRS-1 or Akt/PKB. These effects could contribute to the vicious cycle of insulin resistance and thereby aggravate the existing cardiac impairments. 


\subsubsection{Diacylglycerol formation}

It is known that in obesity and diabetes, due to an oversupply in lipids, DAGs can accumulate in different cellular compartments. It is hypothesized that DAGs interfere with the insulin signaling cascade in the heart as they do in skeletal muscle ${ }^{115}$, leading to a decreased insulin stimulated glucose uptake. Increased accumulation of DAG activates PKCE and $\delta$, which in turn interfere with the insulin-signaling cascade. A 10-week high fat diet in C57BL/6 mice, resulted in a decreased insulin-stimulated glucose oxidation in isolated working hearts. This was positively associated with increased accumulation of myocardial DAG, a concomitantly increase in GPAT and a decrease in DGAT, as well as an inhibition of insulin-signaling molecules ${ }^{116}$.

Whether DAG can also exert direct lipotoxic effects by inducing apoptosis in cardiac tissue remains unknown. Overexpression of DGAT in the heart reduced DAG levels and had a cardioprotective effect when crossed with a mouse model of cardiac lipotoxicity. However, in this model neutral lipid storage was increased. Besides the decrase in DAG levels, ceramide formation was decreased by $35 \%$. Therefore it remains unclear whether the cardioprotective effect was due to the lowering of DAG or ceramides levels ${ }^{54}$.

\subsubsection{Membrane destabilisation and ER-stress}

Another potential lipotoxic mechanism that may be involved in diabetic cardiomyopathy is membrane destabilization and endoplasmic reticulum (ER)-stress ${ }^{117}$. The ER is a primary site for protein synthesis and folding. Most secreted and transmembrane proteins fold and mature in the lumen of the ER. Cells can adjust the protein-folding capacity of the ER, thereby ensuring that the nessecary proteins can maintain cellular function in due time. The intracellular signalling pathway that mediates this regulation has been named the unfolded protein response (UFPR) ${ }^{118}$.

In obesity, increased lipid delivery can stimulate an increased influx of unfolded proteins in the ER, which can lead to a mismatch between the UFPR and protein translation, inducing stress of the ER. ${ }^{118}$ ER stress enhances calcium release and signaling to the mitochondria, ultimately resulting in increased apoptosis. ${ }^{119}$

In mice with streptozotocin-induced diabetes, cardiac ER-stress was suggested by expression of ER chaperones and apoptosis was detected five months after diabetes onset. Also, in models of type 2 diabetes it was shown that cardiomyopathy was associated with ER-stress. In $d b / d b$ mice levels of the phosphorylated ER stress makers were significantly elevated compared with lean controls ${ }^{120}$. It has been shown in vitro that ER-stress is enhanced in lipotoxicity. Cells exposed to palmitate could rapidly converted it into saturated phospholipids, which in turn integrated into the microsomal membranes. This resulted in 
drastic membrane remodeling which was associated with dramatic dilatation of the ER and redistribution of protein-folding chaperones to the cytosol within five hours, indicating compromised ER membrane integrity and further enhancing ER-stress ${ }^{121}$. Although there is no data on ER-stress in human cardiac tissue, data from animal studies strongly suggest that indeed ER-stress is involved in the induction of apoptosis and that this mechanism is enhanced by lipid accumulation.

\subsubsection{Systemic and local inflammation}

It is well established that obesity and type 2 diabetes are characterized by a state of lowgrade inflammation ${ }^{122,123}$. Systemic low grade inflammation influences cellular processes in all tissues, including the heart and may lead to fibrosis and structural remodelling which may contribute to the cardiac stiffness/diastolic impairment observed in diabetic cardiomyopathy.

Epicardial adipose tissue has been identified as an active source of adipokines and cytokines $^{124}$. Due to the close relationship between this fat depot and the myocardial tissue, coupled with the lack of fascial boundaries, epicardial adipose tissue may locally interact and modulate the myocardium through secretion of pro- and anti-inflammatory cytokines ${ }^{125}$. Epicardial adipose tissue serves as a buffer for the storage of free fatty acids for the heart. However, the increased epicardial thickness as seen in obesity, has been associated with proinflammatory cytokine production ${ }^{126}$. Thereby, epicardial adipose tissue derived cytokines may significantly contribute to the development of diabetic cardiomyopathy.

\section{Implications and conclusions}

In conclusion, accumulation of fat and a higher availability of free fatty acids are associated with an impaired cardiac efficiency and lipoapoptosis. Though many of the theories are confirmed in animal models, the evidence from human studies remains scarce. Nevertheless, the available data points toward the need for lipid lowering strategies to reduce ectopic fat accumulation and minimize lipotoxicity. To unravel the etiology of diabetic cardiomyopathy in humans, novel non-invasive imaging techniques will become increasingly important for in vivo investigations.

\section{Funding}

T.W. was supported by the Center for Translational Molecular Medicine, the Netherlands Heart Foundation, Dutch Diabetes Research Foundation, Dutch Kidney Foundation 
(PREDICCt). V.S. and P.S. were supported by a veni and a vici grant, respectively, from the Netherlands organization for scientific research (NWO).

\section{Figure 2: Possible mechanisms leading to lipoapoptosis in cardiomyocytes}

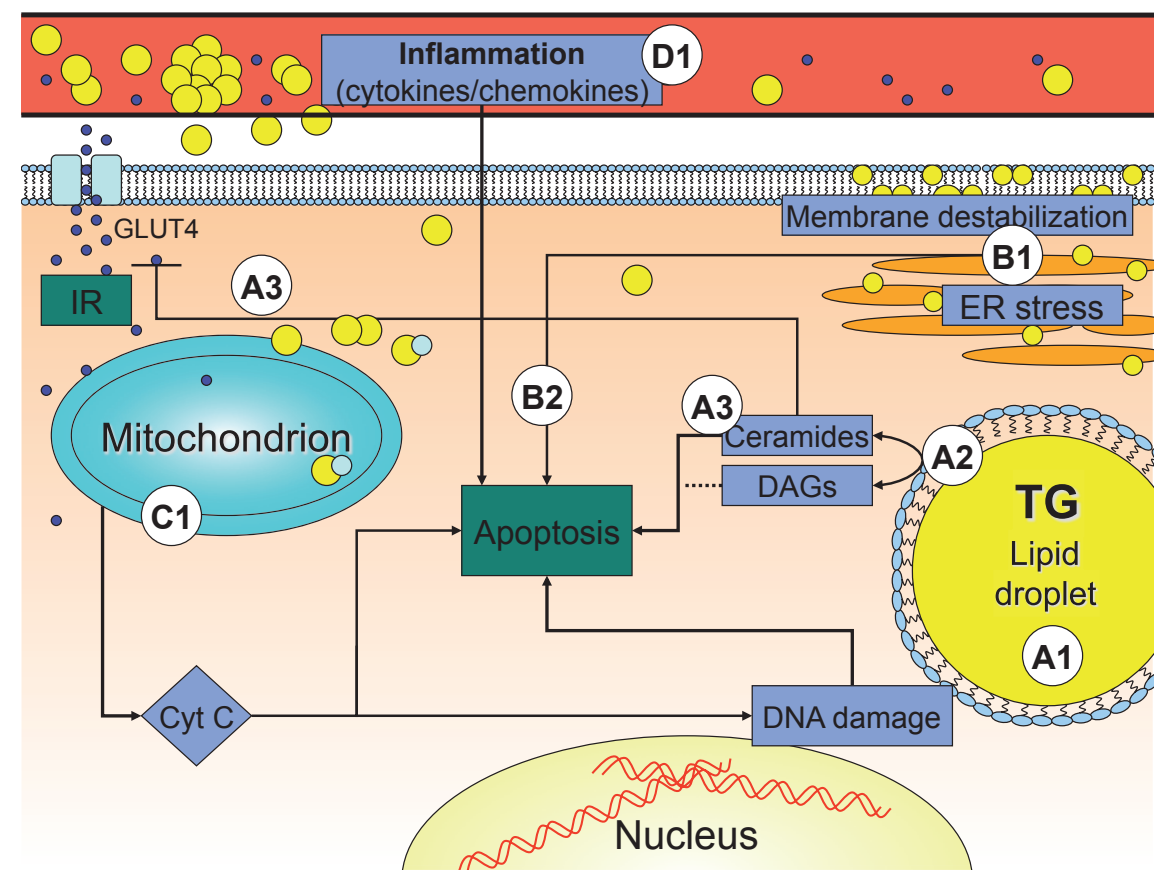

(A1) Stored triglycerides in cardiomyocytes can be hydrolyzed, resulting in (A2) diacylglycerols and fatty acids that can be metabolized in non-oxidative pathways. In that way, saturated fatty acids can be converted into ceramides. Ceramides can initiate lipoapoptotic pathways (A3) via cytochrome $C$ release of the mitochondria and can inhibit the insulin-signaling pathway via PKC resulting in IR. DAGs also induce IR via interference with the insulin signaling pathways, but whether DAGs can also induce apoptosis in cardiac muscle still remains unknown. Lipids that cannot be stored can also be integrated as saturated phospholipids in the membranes and induce imbalances in the UFPR leading to ER stress (B1), which in turn (B2) can lead via mitochondrial pathways (C1) and cytochrome $C$ release to lipoapoptosis. Also cytokines from systemic and locally inflamed adipose tissue (D1.), might induce cellular signaling and attract immune cells thereby inducing local inflammation leading to insulin resistance, cellular damage and apoptosis 


\section{References}

1. Garcia MJ, McNamara PM, Gordon T, Kannel WB. Morbidity and mortality in diabetics in the Framingham population. Sixteen year follow-up study. Diabetes 1974;23:105-111.

2. Haffner SM, Lehto S, Ronnemaa T, Pyorala K, Laakso M. Mortality from coronary heart disease in subjects with type 2 diabetes and in nondiabetic subjects with and without prior myocardial infarction. N Engl J Med 1998;339:229-234.

3. Rubler S, Dlugash J, Yuceoglu YZ, Kumral T, Branwood AW, Grishman A. New type of cardiomyopathy associated with diabetic glomerulosclerosis. Am J Cardiol 1972;30:595-602.

4. Kannel WB, Hjortland M, Castelli WP. Role of diabetes in congestive heart failure: the Framingham study. Am J Cardiol 1974;34:29-34.

5. Fein FS, Sonnenblick EH. Diabetic cardiomyopathy. Prog Cardiovasc Dis 1985;27:255-270.

6. Unger RH. Lipotoxicity in the pathogenesis of obesity-dependent NIDDM. Genetic and clinical implications. Diabetes 1995;44:863-870.

7. Liu JE, Palmieri V, Roman MJ, Bella JN, Fabsitz R, Howard BV, et al. The impact of diabetes on left ventricular filling pattern in normotensive and hypertensive adults: the Strong Heart Study. J Am Coll Cardiol 2001;37:1943-1949.

8. Bell DS. Diabetic cardiomyopathy. Diabetes Care 2003;26:2949-2951.

9. Poirier P, Bogaty P, Garneau C, Marois L, Dumesnil JG. Diastolic dysfunction in normotensive men with well-controlled type 2 diabetes: importance of maneuvers in echocardiographic screening for preclinical diabetic cardiomyopathy. Diabetes Care 2001;24:5-10.

10. Zabalgoitia M, Ismaeil MF, Anderson L, Maklady FA. Prevalence of diastolic dysfunction in normotensive, asymptomatic patients with well-controlled type 2 diabetes mellitus. Am J Cardiol 2001;87:320-323.

11. McGavock JM, Lingvay I, Zib I, Tillery T, Salas N, Unger R, et al. Cardiac steatosis in diabetes mellitus: a 1H-magnetic resonance spectroscopy study. Circulation 2007;116:1170-1175.

12. McGavock JM, Victor RG, Unger RH, Szczepaniak LS. Adiposity of the heart, revisited. Ann Intern Med 2006;144:517-524.

13. Singh S, Dhingra S, Ramdath DD, Vasdev S, Gill V, Singal PK. Risk factors preceding type 2 diabetes and cardiomyopathy. J Cardiovasc Transl Res;3:580-596.

14. Despres JP, Lemieux I, Bergeron J, Pibarot P, Mathieu P, Larose E, et al. Abdominal obesity and the metabolic syndrome: contribution to global cardiometabolic risk. Arterioscler Thromb Vasc Biol 2008;28:1039-1049.

15. Lakka TA, Bouchard C. Physical activity, obesity and cardiovascular diseases. Handb Exp Pharmacol 2005:137-163.

16. Boden G. Obesity, insulin resistance and free fatty acids. Curr Opin Endocrinol Diabetes Obes 2011.

17. Spector KS. Diabetic cardiomyopathy. Clin Cardiol 1998;21:885-887.

18. Boudina S, Abel ED. Diabetic cardiomyopathy revisited. Circulation 2007;115:3213-3223.

19. Lopaschuk GD. Abnormal mechanical function in diabetes: relationship to altered myocardial carbohydrate/lipid metabolism. Coron Artery Dis 1996;7:116-123.

20. Rijzewijk LJ, van der Meer RW, Lamb HJ, de Jong HW, Lubberink M, Romijn JA, et al. Altered myocardial substrate metabolism and decreased diastolic function in nonischemic human diabetic cardiomyopathy: studies with cardiac positron emission tomography and magnetic resonance imaging. J Am Coll Cardiol 2009;54:1524-1532. 
21. Anderson EJ, Kypson AP, Rodriguez E, Anderson CA, Lehr EJ, Neufer PD. Substrate-specific derangements in mitochondrial metabolism and redox balance in the atrium of the type 2 diabetic human heart. J Am Coll Cardiol 2009;54:1891-1898.

22. Normand-Lauziere F, Frisch F, Labbe SM, Bherer P, Gagnon R, Cunnane SC, et al. Increased postprandial nonesterified fatty acid appearance and oxidation in type 2 diabetes is not fully established in offspring of diabetic subjects. PLoS One 2010;5:e10956.

23. II'yasova D, Wang F, D'Agostino RB, Jr., Hanley A, Wagenknecht LE. Prospective association between fasting NEFA and type 2 diabetes: impact of post-load glucose. Diabetologia 2010;53:866-874.

24. Cusi K. The role of adipose tissue and lipotoxicity in the pathogenesis of type 2 diabetes. Curr Diab Rep;10:306-315.

25. Lionetti L, Mollica MP, Lombardi A, Cavaliere G, Gifuni G, Barletta A. From chronic overnutrition to insulin resistance: the role of fat-storing capacity and inflammation. Nutr Metab Cardiovasc Dis 2009;19:146-152.

26. Szczepaniak LS, Dobbins RL, Metzger GJ, Sartoni-D’Ambrosia G, Arbique D, Vongpatanasin W, et al. Myocardial triglycerides and systolic function in humans: in vivo evaluation by localized proton spectroscopy and cardiac imaging. Magn Reson Med 2003;49:417-423.

27. Sharma S, Adrogue JV, Golfman L, Uray I, Lemm J, Youker K, et al. Intramyocardial lipid accumulation in the failing human heart resembles the lipotoxic rat heart. Faseb $J$ 2004;18:1692-1700.

28. Kankaanpaa M, Lehto HR, Parkka JP, Komu M, Viljanen A, Ferrannini E, et al. Myocardial triglyceride content and epicardial fat mass in human obesity: relationship to left ventricular function and serum free fatty acid levels. J Clin Endocrinol Metab 2006;91:4689-4695.

29. Christoffersen C, Bollano E, Lindegaard ML, Bartels ED, Goetze JP, Andersen CB, et al. Cardiac lipid accumulation associated with diastolic dysfunction in obese mice. Endocrinology 2003;144:3483-3490.

30. Hammer S, van der Meer RW, Lamb HJ, Schar M, de Roos A, Smit JW, et al. Progressive caloric restriction induces dose-dependent changes in myocardial triglyceride content and diastolic function in healthy men. J Clin Endocrinol Metab 2008;93:497-503.

31. Bilet L, van de Weijer T, Hesselink MK, Glatz JF, Lamb HJ, Wildberger J, et al. Exercise-induced modulation of cardiac lipid content in healthy lean young men. Basic Res Cardiol 2011.

32. Hamilton JA, Guo W, Kamp F. Mechanism of cellular uptake of long-chain fatty acids: Do we need cellular proteins? Mol Cell Biochem 2002;239:17-23.

33. Sprong $\mathrm{H}$, van der Sluijs $\mathrm{P}$, van Meer $\mathrm{G}$. How proteins move lipids and lipids move proteins. Nat Rev Mol Cell Biol 2001;2:504-513.

34. Glatz JF, Luiken JJ, Bonen A. Membrane fatty acid transporters as regulators of lipid metabolism: implications for metabolic disease. Physiol Rev 2010;90:367-417.

35. Luiken JJ, Turcotte LP, Bonen A. Protein-mediated palmitate uptake and expression of fatty acid transport proteins in heart giant vesicles. J Lipid Res 1999;40:1007-1016.

36. Luiken JJ, Coort SL, Willems J, Coumans WA, Bonen A, van der Vusse GJ, et al. Contractioninduced fatty acid translocase/CD36 translocation in rat cardiac myocytes is mediated through AMP-activated protein kinase signaling. Diabetes 2003;52:1627-1634.

37. Bonen A, Campbell SE, Benton CR, Chabowski A, Coort SL, Han XX, et al. Regulation of fatty acid transport by fatty acid translocase/CD36. Proc Nutr Soc 2004;63:245-249.

38. Ibrahimi A, Bonen A, Blinn WD, Hajri T, Li X, Zhong K, et al. Muscle-specific overexpression of FAT/CD36 enhances fatty acid oxidation by contracting muscle, reduces plasma triglycerides 
and fatty acids, and increases plasma glucose and insulin. J Biol Chem 1999;274:2676126766.

39. Luiken JJ, Coort SL, Willems J, Coumans WA, Bonen A, Glatz JF. Dipyridamole alters cardiac substrate preference by inducing translocation of FAT/CD36, but not that of GLUT4. Mol Pharmacol 2004;65:639-645.

40. Lopaschuk GD, Ussher JR, Folmes CD, Jaswal JS, Stanley WC. Myocardial fatty acid metabolism in health and disease. Physiol Rev 2010;90:207-258.

41. Coort SL, Hasselbaink DM, Koonen DP, Willems J, Coumans WA, Chabowski A, et al. Enhanced sarcolemmal FAT/CD36 content and triacylglycerol storage in cardiac myocytes from obese zucker rats. Diabetes 2004;53:1655-1663.

42. Coort SL, Luiken JJ, van der Vusse GJ, Bonen A, Glatz JF. Increased FAT (fatty acid translocase)/ CD36-mediated long-chain fatty acid uptake in cardiac myocytes from obese Zucker rats. Biochem Soc Trans 2004;32:83-85.

43. Carley AN, Severson DL. Fatty acid metabolism is enhanced in type 2 diabetic hearts. Biochim Biophys Acta 2005;1734:112-126.

44. Chiu HC, Kovacs A, Blanton RM, Han X, Courtois M, Weinheimer CJ, et al. Transgenic expression of fatty acid transport protein 1 in the heart causes lipotoxic cardiomyopathy. Circ Res 2005;96:225-233.

45. Nickerson JG, Momken I, Benton CR, Lally J, Holloway GP, Han XX, et al. Protein-mediated fatty acid uptake: regulation by contraction, AMP-activated protein kinase, and endocrine signals. Appl Physiol Nutr Metab 2007;32:865-873.

46. Nickerson JG, Alkhateeb H, Benton CR, Lally J, Nickerson J, Han XX, et al. Greater transport efficiencies of the membrane fatty acid transporters FAT/CD36 and FATP4 compared with FABPpm and FATP1 and differential effects on fatty acid esterification and oxidation in rat skeletal muscle. J Biol Chem 2009;284:16522-16530.

47. Gimeno RE, Ortegon AM, Patel S, Punreddy S, Ge P, Sun Y, et al. Characterization of a heartspecific fatty acid transport protein. J Biol Chem 2003;278:16039-16044.

48. Lavrentyev EN, He D, Cook GA. Expression of genes participating in regulation of fatty acid and glucose utilization and energy metabolism in developing rat hearts. Am J Physiol Heart Circ Physiol 2004;287:H2035-2042.

49. DiRusso CC, Li H, Darwis D, Watkins PA, Berger J, Black PN. Comparative biochemical studies of the murine fatty acid transport proteins (FATP) expressed in yeast. J Biol Chem 2005;280:16829-16837.

50. Schoonderwoerd K, Broekhoven-Schokker S, Hulsmann WC, Stam H. Properties of phosphatidate phosphohydrolase and diacylglycerol acyltransferase activities in the isolated rat heart. Effect of glucagon, ischaemia and diabetes. Biochem J 1990;268:487-492.

51. Lewin TM, de Jong H, Schwerbrock NJ, Hammond LE, Watkins SM, Combs TP, et al. Mice deficient in mitochondrial glycerol-3-phosphate acyltransferase-1 have diminished myocardial triacylglycerol accumulation during lipogenic diet and altered phospholipid fatty acid composition. Biochim Biophys Acta 2008;1781:352-358.

52. Atkinson LL, Kozak R, Kelly SE, Onay Besikci A, Russell JC, Lopaschuk GD. Potential mechanisms and consequences of cardiac triacylglycerol accumulation in insulin-resistant rats. Am J Physiol Endocrinol Metab 2003;284:E923-930.

53. Glenn DJ, Wang F, Nishimoto M, Cruz MC, Uchida Y, Holleran WM, et al. A murine model of isolated cardiac steatosis leads to cardiomyopathy. Hypertension 2011;57:216-222. 
54. Liu L, Shi X, Bharadwaj KG, Ikeda S, Yamashita H, Yagyu H, et al. DGAT1 expression increases heart triglyceride content but ameliorates lipotoxicity. J Biol Chem 2009;284:36312-36323.

55. Ueno M, Suzuki J, Zenimaru Y, Takahashi S, Koizumi T, Noriki S, et al. Cardiac overexpression of hormone-sensitive lipase inhibits myocardial steatosis and fibrosis in streptozotocin diabetic mice. Am J Physiol Endocrinol Metab 2008;294:E1109-1118.

56. Haemmerle G, Lass A, Zimmermann R, Gorkiewicz G, Meyer C, Rozman J, et al. Defective lipolysis and altered energy metabolism in mice lacking adipose triglyceride lipase. Science 2006;312:734-737.

57. Lass A, Zimmermann R, Oberer M, Zechner R. Lipolysis - a highly regulated multi-enzyme complex mediates the catabolism of cellular fat stores. Prog Lipid Res 2011;50:14-27.

58. Fischer J, Lefevre C, Morava E, Mussini JM, Laforet P, Negre-Salvayre A, et al. The gene encoding adipose triglyceride lipase (PNPLA2) is mutated in neutral lipid storage disease with myopathy. Nat Genet 2007;39:28-30.

59. Belke DD, Larsen TS, Gibbs EM, Severson DL. Altered metabolism causes cardiac dysfunction in perfused hearts from diabetic (db/db) mice. Am J Physiol Endocrinol Metab 2000;279:E11041113.

60. Isenberg G, Han S, Schiefer A, Wendt-Gallitelli MF. Changes in mitochondrial calcium concentration during the cardiac contraction cycle. Cardiovasc Res 1993;27:1800-1809.

61. Wu P, Peters JM, Harris RA. Adaptive increase in pyruvate dehydrogenase kinase 4 during starvation is mediated by peroxisome proliferator-activated receptor alpha. Biochem Biophys Res Commun 2001;287:391-396.

62. Chatham JC, Forder JR. Metabolic compartmentation of lactate in the glucose-perfused rat heart. Am J Physiol 1996;270:H224-229.

63. Boudina S, Sena S, Theobald H, Sheng X, Wright JJ, Hu XX, et al. Mitochondrial energetics in the heart in obesity-related diabetes: direct evidence for increased uncoupled respiration and activation of uncoupling proteins. Diabetes 2007;56:2457-2466.

64. Lopaschuk GD, Spafford M. Response of isolated working hearts to fatty acids and carnitine palmitoyltransferase I inhibition during reduction of coronary flow in acutely and chronically diabetic rats. Circ Res 1989;65:378-387.

65. Randle PJ, Garland PB, Hales CN, Newsholme EA. The glucose fatty-acid cycle. Its role in insulin sensitivity and the metabolic disturbances of diabetes mellitus. Lancet 1963;1:785789.

66. Finck BN, Lehman JJ, Leone TC, Welch MJ, Bennett MJ, Kovacs A, et al. The cardiac phenotype induced by PPARalpha overexpression mimics that caused by diabetes mellitus. J Clin Invest 2002;109:121-130.

67. Young ME, Patil S, Ying J, Depre C, Ahuja HS, Shipley GL, et al. Uncoupling protein 3 transcription is regulated by peroxisome proliferator-activated receptor (alpha) in the adult rodent heart. Faseb J 2001;15:833-845.

68. Zhou YT, Grayburn P, Karim A, Shimabukuro M, Higa M, Baetens D, et al. Lipotoxic heart disease in obese rats: implications for human obesity. Proc Natl Acad Sci U S A 2000;97:17841789.

69. Marfella R, Di Filippo C, Portoghese M, Barbieri M, Ferraraccio F, Siniscalchi M, et al. Myocardial lipid accumulation in patients with pressure-overloaded heart and metabolic syndrome. J Lipid Res 2009;50:2314-2323.

70. Dewald O, Sharma S, Adrogue J, Salazar R, Duerr GD, Crapo JD, et al. Downregulation of peroxisome proliferator-activated receptor-alpha gene expression in a mouse model of 
ischemic cardiomyopathy is dependent on reactive oxygen species and prevents lipotoxicity. Circulation 2005;112:407-415.

71. Nuutila P, Koivisto VA, Knuuti J, Ruotsalainen U, Teras M, Haaparanta M, et al. Glucosefree fatty acid cycle operates in human heart and skeletal muscle in vivo. J Clin Invest 1992;89:1767-1774.

72. Zachara NE, Hart GW. O-GlcNAc a sensor of cellular state: the role of nucleocytoplasmic glycosylation in modulating cellular function in response to nutrition and stress. Biochim Biophys Acta 2004;1673:13-28.

73. Wells L, Hart GW. O-GlcNAc turns twenty: functional implications for post-translational modification of nuclear and cytosolic proteins with a sugar. FEBS Lett 2003;546:154-158.

74. Rajamani U, Essop MF. Hyperglycemia-mediated activation of the hexosamine biosynthetic pathway results in myocardial apoptosis. Am J Physiol Cell Physiol;299:C139-147.

75. Stanley WC, Lopaschuk GD, McCormack JG. Regulation of energy substrate metabolism in the diabetic heart. Cardiovasc Res 1997;34:25-33.

76. Paulson DJ. The diabetic heart is more sensitive to ischemic injury. Cardiovasc Res 1997;34:104-112.

77. Opie LH. The metabolic vicious cycle in heart failure. Lancet 2004;364:1733-1734.

78. Burkhoff D, Weiss RG, Schulman SP, Kalil-Filho R, Wannenburg T, Gerstenblith G. Influence of metabolic substrate on rat heart function and metabolism at different coronary flows. Am J Physiol 1991;261:H741-750.

79. Liu Q, Docherty JC, Rendell JC, Clanachan AS, Lopaschuk GD. High levels of fatty acids delay the recovery of intracellular $\mathrm{pH}$ and cardiac efficiency in post-ischemic hearts by inhibiting glucose oxidation. J Am Coll Cardiol 2002;39:718-725.

80. Taegtmeyer $\mathrm{H}, \mathrm{McNulty} \mathrm{P}$, Young ME. Adaptation and maladaptation of the heart in diabetes: Part I: general concepts. Circulation 2002;105:1727-1733.

81. Boehm EA, Jones BE, Radda GK, Veech RL, Clarke K. Increased uncoupling proteins and decreased efficiency in palmitate-perfused hyperthyroid rat heart. Am J Physiol Heart Circ Physiol 2001;280:H977-983.

82. Murray AJ, Anderson RE, Watson GC, Radda GK, Clarke K. Uncoupling proteins in human heart. Lancet 2004;364:1786-1788.

83. Schrauwen $\mathrm{P}$, Saris WH, Hesselink MK. An alternative function for human uncoupling protein 3: protection of mitochondria against accumulation of nonesterified fatty acids inside the mitochondrial matrix. Faseb J 2001;15:2497-2502.

84. Tune JD, Yeh C, Setty S, Downey HF. ATP-dependent $\mathrm{K}(+)$ channels contribute to local metabolic coronary vasodilation in experimental diabetes. Diabetes 2002;51:1201-1207.

85. Wojtczak L, Schonfeld P. Effect of fatty acids on energy coupling processes in mitochondria. Biochim Biophys Acta 1993;1183:41-57.

86. Samartsev VN, Smirnov AV, Zeldi IP, Markova OV, Mokhova EN, Skulachev VP. Involvement of aspartate/glutamate antiporter in fatty acid-induced uncoupling of liver mitochondria. Biochim Biophys Acta 1997;1319:251-257.

87. Skulachev VP. Membrane-linked systems preventing superoxide formation. Biosci Rep 1997;17:347-366.

88. Starkov AA. "Mild" uncoupling of mitochondria. Biosci Rep 1997;17:273-279.

89. Knuuti J, Takala TO, Nagren K, Sipila H, Turpeinen AK, Uusitupa MI, et al. Myocardial fatty acid oxidation in patients with impaired glucose tolerance. Diabetologia 2001;44:184-187. 
90. Veerappan RM, Senthil S, Rao MR, Ravikumar R, Pugalendi KV. Redox status and lipid peroxidation in alcoholic hypertensive patients and alcoholic hypertensive patients with diabetes. Clin Chim Acta 2004;340:207-212.

91. Cimbaljevic B, Vasilijevic A, CimbaljevicS, Buzadzic B, Korac A, Petrovic V, et al. Interrelationship of antioxidative status, lipid peroxidation, and lipid profile in insulin-dependent and noninsulin-dependent diabetic patients. Can J Physiol Pharmacol 2007;85:997-1003.

92. Song Y, Du Y, Prabhu SD, Epstein PN. Diabetic Cardiomyopathy in OVE26 Mice Shows Mitochondrial ROS Production and Divergence Between In Vivo and In Vitro Contractility. Rev Diabet Stud 2007;4:159-168.

93. Boudina S, Sena S, O'Neill BT, Tathireddy P, Young ME, Abel ED. Reduced mitochondrial oxidative capacity and increased mitochondrial uncoupling impair myocardial energetics in obesity. Circulation 2005;112:2686-2695.

94. Lin J, Yang R, Tarr PT, Wu PH, Handschin C, Li S, et al. Hyperlipidemic effects of dietary saturated fats mediated through PGC-1beta coactivation of SREBP. Cell 2005;120:261-273.

95. Boudina S, Bugger H, Sena S, O'Neill BT, Zaha VG, Ilkun O, et al. Contribution of impaired myocardial insulin signaling to mitochondrial dysfunction and oxidative stress in the heart. Circulation 2009;119:1272-1283.

96. Tanaka Y, Konno N, Kako KJ. Mitochondrial dysfunction observed in situ in cardiomyocytes of rats in experimental diabetes. Cardiovasc Res 1992;26:409-414.

97. How OJ, Aasum E, Severson DL, Chan WY, Essop MF, Larsen TS. Increased myocardial oxygen consumption reduces cardiac efficiency in diabetic mice. Diabetes 2006;55:466-473.

98. How OJ, Aasum E, Kunnathu S, Severson DL, Myhre ES, Larsen TS. Influence of substrate supply on cardiac efficiency, as measured by pressure-volume analysis in ex vivo mouse hearts. Am J Physiol Heart Circ Physiol 2005;288:H2979-2985.

99. Scheuermann-Freestone M, Madsen PL, Manners D, Blamire AM, Buckingham RE, Styles $\mathrm{P}$, et al. Abnormal cardiac and skeletal muscle energy metabolism in patients with type 2 diabetes. Circulation 2003;107:3040-3046.

100. Diamant M, Lamb HJ, Groeneveld Y, Endert EL, Smit JW, Bax JJ, et al. Diastolic dysfunction is associated with altered myocardial metabolism in asymptomatic normotensive patients with well-controlled type 2 diabetes mellitus. J Am Coll Cardiol 2003;42:328-335.

101. Nakae I, Mitsunami K, Yoshino T, Omura T, Tsutamoto T, Matsumoto T, et al. Clinical features of myocardial triglyceride in different types of cardiomyopathy assessed by proton magnetic resonance spectroscopy: comparison with myocardial creatine. J Card Fail 2010;16:812-822.

102. van der Meer RW, Rijzewijk LJ, de Jong HW, Lamb HJ, Lubberink M, Romijn JA, et al. Pioglitazone improves cardiac function and alters myocardial substrate metabolism without affecting cardiac triglyceride accumulation and high-energy phosphate metabolism in patients with well-controlled type 2 diabetes mellitus. Circulation 2009;119:2069-2077.

103. Balaban RS. Cardiac energy metabolism homeostasis: role of cytosolic calcium. J Mol Cell Cardiol 2002;34:1259-1271.

104. Belke DD, Swanson EA, Dillmann WH. Decreased sarcoplasmic reticulum activity and contractility in diabetic $\mathrm{db} / \mathrm{db}$ mouse heart. Diabetes 2004;53:3201-3208.

105. Dong F, Zhang X, Yang X, Esberg LB, Yang H, Zhang Z, et al. Impaired cardiac contractile function in ventricular myocytes from leptin-deficient ob/ob obese mice. J Endocrinol 2006;188:25-36. 
106. Fauconnier J, Lanner JT, Zhang SJ, Tavi P, Bruton JD, Katz A, et al. Insulin and inositol 1,4,5-trisphosphate trigger abnormal cytosolic $\mathrm{Ca} 2+$ transients and reveal mitochondrial Ca2+ handling defects in cardiomyocytes of ob/ob mice. Diabetes 2005;54:2375-2381.

107. Relling DP, Esberg LB, Fang CX, Johnson WT, Murphy EJ, Carlson EC, et al. High-fat dietinduced juvenile obesity leads to cardiomyocyte dysfunction and upregulation of Foxo3a transcription factor independent of lipotoxicity and apoptosis. J Hypertens 2006;24:549-561.

108. de Vries JE, Vork MM, Roemen TH, de Jong YF, Cleutjens JP, van der Vusse GJ, et al. Saturated but not mono-unsaturated fatty acids induce apoptotic cell death in neonatal rat ventricular myocytes. J Lipid Res 1997;38:1384-1394.

109. Listenberger LL, Ory DS, Schaffer JE. Palmitate-induced apoptosis can occur through a ceramide-independent pathway. J Biol Chem 2001;276:14890-14895.

110. Miller TA, LeBrasseur NK, Cote GM, Trucillo MP, Pimentel DR, Ido Y, et al. Oleate prevents palmitate-induced cytotoxic stress in cardiac myocytes. Biochem Biophys Res Commun 2005;336:309-315.

111. Bick RJ, Wood DE, Poindexter B, McMillin JB, Karoly A, Wang D, et al. Cytokines increase neonatal cardiac myocyte calcium concentrations: the involvement of nitric oxide and cyclic nucleotides. J Interferon Cytokine Res 1999;19:645-653.

112. Hickson-Bick DL, Buja LM, McMillin JB. Palmitate-mediated alterations in the fatty acid metabolism of rat neonatal cardiac myocytes. J Mol Cell Cardiol 2000;32:511-519.

113. Park TS, Hu Y, Noh HL, Drosatos K, Okajima K, Buchanan J, et al. Ceramide is a cardiotoxin in lipotoxic cardiomyopathy. J Lipid Res 2008;49:2101-2112.

114. Baranowski M, Blachnio-Zabielska A, Hirnle T, Harasiuk D, Matlak K, Knapp M, et al. Myocardium of type 2 diabetic and obese patients is characterized by alterations in sphingolipid metabolic enzymes but not by accumulation of ceramide. J Lipid Res 2010;51:7480.

115. van Herpen NA, Schrauwen-Hinderling VB. Lipid accumulation in non-adipose tissue and lipotoxicity. Physiol Behav 2008;94:231-241.

116. Zhang L, Ussher JR, Oka T, Cadete VJ, Wagg C, Lopaschuk GD. Cardiac Diacylglycerol Accumulation in High Fat-Fed Mice Is Associated with Impaired Insulin-Stimulated Glucose Oxidation. Cardiovasc Res 2011:148-156.

117. Karaskov E, Scott C, Zhang L, Teodoro T, Ravazzola M, Volchuk A. Chronic palmitate but not oleate exposure induces endoplasmic reticulum stress, which may contribute to INS-1 pancreatic beta-cell apoptosis. Endocrinology 2006;147:3398-3407.

118. Ron D, Walter P. Signal integration in the endoplasmic reticulum unfolded protein response. Nat Rev Mol Cell Biol 2007;8:519-529.

119. Szegezdi E, Logue SE, Gorman AM, Samali A. Mediators of endoplasmic reticulum stressinduced apoptosis. EMBO Rep 2006;7:880-885.

120. Dong F, Ren J. Adiponectin improves cardiomyocyte contractile function in $\mathrm{db} / \mathrm{db}$ diabetic obese mice. Obesity (Silver Spring) 2009;17:262-268.

121. Borradaile NM, Han X, Harp JD, Gale SE, Ory DS, Schaffer JE. Disruption of endoplasmic reticulum structure and integrity in lipotoxic cell death. J Lipid Res 2006;47:2726-2737.

122. Karelis $A D$, Faraj $M$, Bastard JP, St-Pierre $D H$, Brochu $M$, Prud'homme $D$, et al. The metabolically healthy but obese individual presents a favorable inflammation profile. J Clin Endocrinol Metab 2005;90:4145-4150. 
123. Bastard JP, Maachi M, Lagathu C, Kim MJ, Caron M, Vidal H, et al. Recent advances in the relationship between obesity, inflammation, and insulin resistance. Eur Cytokine Netw 2006;17:4-12.

124. Mazurek T, Zhang L, Zalewski A, Mannion JD, Diehl JT, Arafat H, et al. Human epicardial adipose tissue is a source of inflammatory mediators. Circulation 2003;108:2460-2466.

125. Iacobellis G, Corradi D, Sharma AM. Epicardial adipose tissue: anatomic, biomolecular and clinical relationships with the heart. Nat Clin Pract Cardiovasc Med 2005;2:536-543.

126. Kremen J, Dolinkova M, Krajickova J, Blaha J, Anderlova K, Lacinova Z, et al. Increased subcutaneous and epicardial adipose tissue production of proinflammatory cytokines in cardiac surgery patients: possible role in postoperative insulin resistance. J Clin Endocrinol Metab 2006;91:4620-4627. 

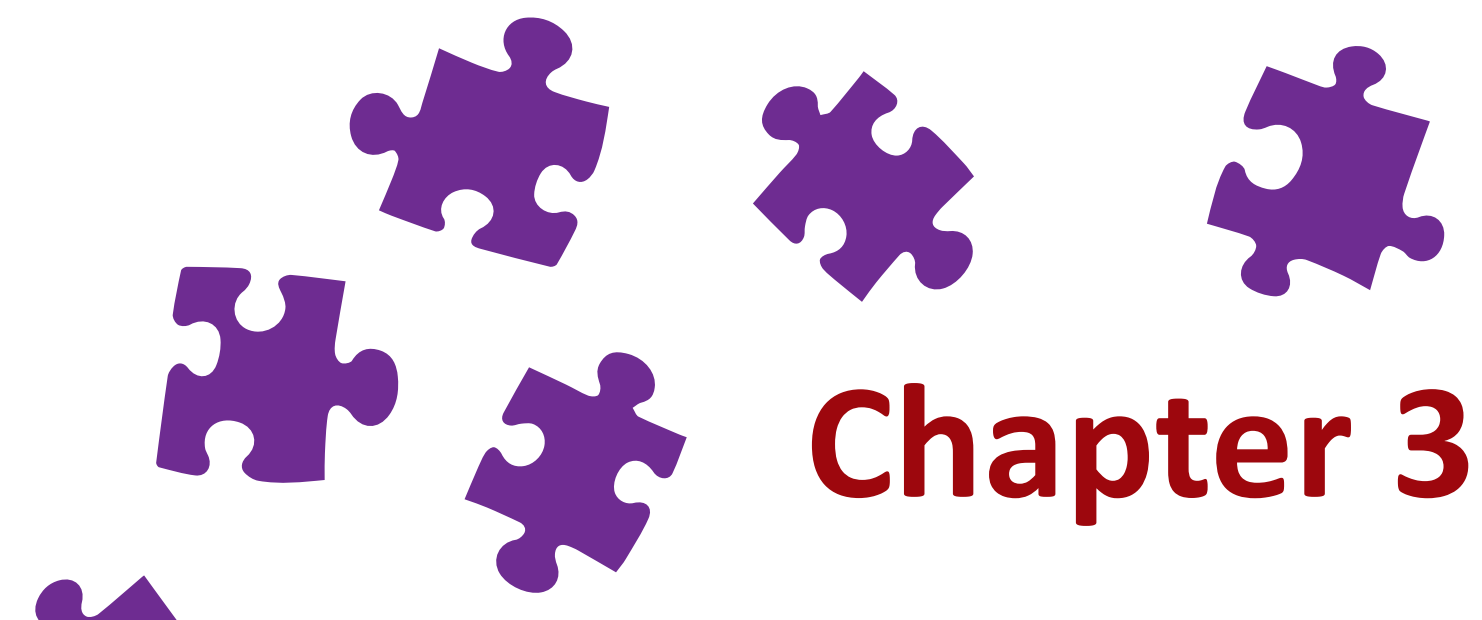

\section{Geometrical models for cardiac MRI in rodents:}

Comparison of quantification of left ventricular volumes and function by various geometrical models with a full-volume data set in rodents. (1)

Tineke van de Weijer ${ }^{1,5}$, Petronella A. van Ewijk ${ }^{1,5}$, H. Reinier Zandbergen ${ }^{6}$, Jos M. Slenter ${ }^{3,6}$, Alfons G. Kessels ${ }^{4}$, Joachim E. Wildberger ${ }^{3,6}$, Matthijs K. C. Hesselink ${ }^{2,5}$, Patrick Schrauwen ${ }^{1,5}$, Vera B. Schrauwen-Hinderling ${ }^{3,5}$, and Marianne Eline Kooi ${ }^{3,5,6}$

Departments of ${ }^{1}$ Human Biology, ${ }^{2}$ Human Movement Sciences, ${ }^{3}$ Radiology and ${ }^{4}$ Clinical Epidemiology, ${ }^{5}$ Nutrim, School for Nutrition, Toxicology and Metabolism, ${ }^{6}$ Cardiovascular Research Institute Maastricht, Maastricht University Medical Centre, The Netherlands 


\section{Abstract}

Background: MRI has been proven to be an accurate method for non-invasive assessment of cardiac function. One of the current limitations of cardiac MRI is that it is time-consuming. Therefore various geometrical models are used, which can reduce scan and post-processing time. It is unclear how appropriate their use is in rodents.

Materials and Methods: LV volumes and ejection fraction (EF) were quantified based on 7.0 Tesla cine-MRI in 12 WT mice, 12 ATGL-/- mice (model of impaired cardiac function) and 11 rats in which we induced cardiac ischemia. The LV volumes and function were either assessed with parallel short-axis slices covering the full volume of the left ventricle (FV, gold standard), or with various geometrical models (modified Simpson rule (SR), biplane ellipsoid (BP), hemisphere cylinder (HC), single-plane ellipsoid (SP) and modified Teichholz Formula (TF)). Reproducibility of the different models was tested and results were correlated with the gold standard (FV).

Results: All models and the FV data set provided reproducible results for the LV volumes and $E F$, with interclass correlation coefficients $\geq 0.87$. All models significantly over- or underestimated EF, except for SR. Good correlation was found for all volumes and EF for the SR model when compared to the FV data set $\left(R^{2}\right.$ ranged between 0.59-0.97 for all parameters). The $\mathrm{HC}$ model and $\mathrm{BP}$ model also predicted EF well $\left(\mathrm{R}^{2} \geq 0.85\right)$, though proved to be less useful for quantitative analysis. The SP and TF models correlated poorly with the $F V$ data set ( $R^{2} \geq 0.45$ for $E F$ and $R^{2} \geq 0.29$ for $E F$, respectively).

Conclusion: For the reduction in acquisition and post-processing time, only the SR model proved to be a valuable method for calculating LV volumes, stroke volume and EF. 


\section{List of abbreviations}

FV Full volume reconstruction model

ATGL Adipose TriGlyceride Lipase

BP Biplane Ellipsoid model

EDV End Diastolic Volume

EF Ejection Fraction

ESV End Systolic Volume

HC Hemisphere Cylinder model

ICC Interclass correlation coefficient

LA Long Axis view

MRI Magnetic Resonance Imaging

SA Short Axis view

SEM Standard error of the mean

SP Single Plane Ellipsoid model

SR modified Simpson Rule model

SV Stroke Volume

TF Modified Teichholz Formula model 


\section{Introduction}

Cardiovascular diseases still are one of the main causes of death in Western countries and the incidence and prevalence of cardiovascular diseases are still increasing ${ }^{1,2}$. Therefore, many studies have focused on the prevention, treatment and etiology of these diseases. Rodent models of cardiac disease have played a critical role in this area of expertise and hence models to assess rodent cardiac function have become increasingly important over the last decades 3,4 .

MRI has been proven to be an accurate method for non-invasive assessment of cardiac function in rodents ${ }^{5-7}$, and has therefore become an important tool in studies of rodent models of cardiac disease ${ }^{8,9}$. With MRI, systolic function can be assessed by calculating the Ejection Fraction (EF) from sequential multi-slice short-axis cine MR images covering the complete volume of the left ventricle $e^{5}$. However, this requires an acquisition of at least 6-8 slices (depending on the size of the heart), which can be quite time consuming, especially in studies requiring high spatial resolution. Also manual or semi-automatic analysis of these slices can demand a substantial amount of time in high-throughput applications ${ }^{10}$. Furthermore, it might be desirable to be able to allow quick assessment of the cardiac function, as a secondary parameter, without increasing scan-time too much. The acquisition duration is not only demanding in terms of scan-time but also prolongs the duration of anesthesia and hence increases stress in the animals under investigation ${ }^{11,12}$. In specific cardiovascular compromised genotypes, such as the adipose triglyceride lipase knock-out (ATGL-/-) mouse, which was used in our studies ${ }^{13}$, prolonged anesthesia can in some cases increase mortality rate.

To circumvent this, several studies have implemented geometrical models for assessment of cardiac function in rodents, rather than acquiring multiple parallel slices with multi slice cine-MR imaging to determine the full volume of the left ventricle ${ }^{14-20}$. These models estimate left ventricular volumes based on single or perpendicular biplane or even tri-plane slices $^{21}$ and therefore allow quick assessment of LV volumes and EF, as they only require acquisition of 1 up to 3 instead of 6-8 slices for mice. For rats, due to the larger size of the left ventricle, the reduction in number of slices is even larger, i.e. 1-3 instead of 6-10 slices. This would substantially reduce total scan time with 10-30 minutes for mice, and even 20-45 minutes for rats. A typical total examination time during which the mice and rats are kept under anesthesia for assessment of cardiac function is 40-45 and 45-60 minutes, respectively. Additionally, acquisition of images required for the geometrical models instead of a full volume data set would reduce the number of slices to be segmented to calculate the 
LV volumes and EF with a factor of 2-3 for mice and 2-4 for rats, thereby also reducing time spent on the assessment of cardiac function in rodent models of cardiac disease.

Although these models have been compared with ultrasound ${ }^{19}$, and have been validated in humans ${ }^{21}$, to our knowledge no study has determined which of these geometrical models is most accurate in determining cardiac function when compared to the gold standard, i.e. cine-MRI of the complete left ventricle by full volume imaging in rodents. Therefore, the

Figure 1: Long axis, 4 chamber view and Short axis images of ATGL-/- and WT mice and a rat model of myocardial infarction.

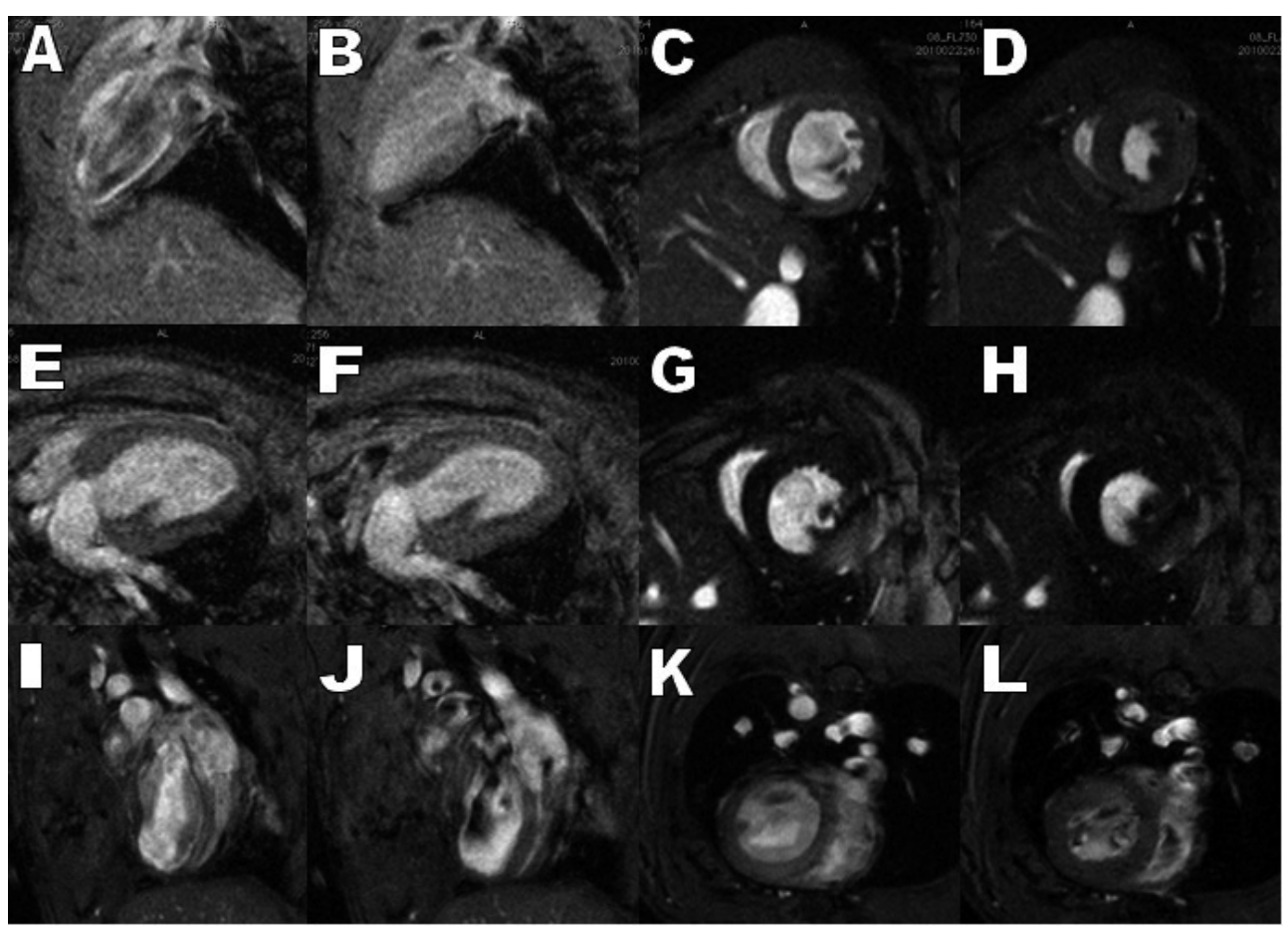

Depicted above are the LA-plane images of the WT mice, A. in diastole and B. in systole. LA-plane image in diastole of a ATGL-/- mice is depicted in figure E, and LA-plane image in a ATGL-/- mice in systole is depicted in figure F. Here a plane through the mitral valve and the apex was chosen to assess LV length and volume. In figure I a $4 \mathrm{CH}$ view is depicted of a rat heart in diastole. Picture $\mathrm{J}$ is a $4 \mathrm{CH}$ view of a rat heart in diastole, after myocardial infarction. In C. and D. SA images are depicted in WT mice, in G. and H. SA images are depicted of a ATGL-/- mice and in picture K. and L. SA images are depicted of a rat heart after myocardial infarction in diastole and systole, respectively. The short axis view was positioned perpendicular to the LA-plane and 4CH view, for the quantification of LV volumes. Furthermore, in these images an impaired systolic contraction and a dilated and thickened ventricular wall is visible in the ATGL-/- mice (middle panels), as well as an infracted area in the left ventricle and slight dilatation of the rat heart (lower panels). 
aim of the present study was to evaluate reproducibility and validity of these geometrical models in mice compared to the gold standard, cine-MRI of the complete left ventricle. After testing the validity of these geometrical models in wild type mice with anticipated normal cardiac function, we tested applicability of these models in mice with anticipated cardiac failure, and in rats in which we induced myocardial infarction. 


\section{Methods}

\section{Animals}

Mice and rats were housed under standard conditions at $25{ }^{\circ} \mathrm{C}$ with a $14: 10 \mathrm{~h}$ light/dark cycle with ad libitum access to water and standard chow diet. The institutional ethics committee on animal welfare approved all experiments. Twelve wild type C57BL/6 mice with anticipated normal cardiac function were imaged for validation of the geometrical models. In addition, we extended the validation to a model of severe cardiac dysfunction by using 12 Adipose TriGlyceride Lipase knock-out mice (ATGL-/-, age 8-12 weeks). ATGL-/- mice were generated on a mixed genetic background (50\% C57BL/6 and 50\% 129/Ola) as previously described ${ }^{22}$. The targeted ATGL allele was then backcrossed onto the C57BL/6 background strain for $>10$ generations. Furthermore, myocardial infarction (MI) was induced in 11 male Wistar-rats (300-500 gram) by ligation of the left anterior descending coronary artery using a 6-0 Prolene suture, as described previously ${ }^{23}, 24$. Subsequently, the rats were scanned between 7 and 21 days post-MI.

Prior to MRI, mice were anesthetized using 1-2\% isoflurane (Abbott Laboratories Ltd, Queensborough, UK) in medical air. Neonatal ECG electrodes (3M, St Paul, MN) were placed on the paws of the right front leg and left hind leg and connected to an MR compatible small animal monitoring system (SA Instruments, Stony Brook, NY). Animals were placed on a warm waterbed. Respiratory rate was continuously monitored.

\section{MRI protocol}

MRI was performed on a 7 Tesla Bruker Biospec 70/30 USR (Bruker Biospin GmbH, Ettlingen, Germany) using the BGA12-S mini-imaging gradient (maximum gradient strength $400 \mathrm{mTm}$ 1 , slew rate $5000 \mathrm{Tm}^{-1} \mathrm{~s}^{-1}$, linear inductive rise time 5-95\% on all axis; $80 \mu \mathrm{s}$ ), interfaced to an AVANCE II console. First, a bright blood cine image with 10 cardiac phases was recorded in horizontal 4 chamber view $(4 \mathrm{CH})$ using a retrospectively self-gated protocol (IntraGate ${ }^{\mathrm{TM}}$, Bruker Biospin, imaging time approximately 2-4 minutes, for the mice: $2.56 \times 2.56 \mathrm{~cm}^{2}$ fieldof-view, 164x164 matrix size; for the rats: $5.0 \times 5.0 \mathrm{~cm}^{2}$ field-of-view, 128x128 matrix size; (see figure $1 \mathrm{l}$ and J)). This $4 \mathrm{CH}$ orientation was used to plan a long axis view (LA) perpendicular to the $4 \mathrm{CH}$ view with the same self-gated protocol, see figure $1 \mathrm{~A}, \mathrm{~B}, \mathrm{E}$ and $\mathrm{F}$. The orientation was defined as a sagittal plane through the mitral valve and the apex. Perpendicular to this LA and $4 \mathrm{CH}$ view and the septum, a short axis view (SA) was positioned (see figure $1 \mathrm{C}, \mathrm{D}, \mathrm{G}, \mathrm{H}$, $K$ and $L$ ). The slice was planned at half height of the left ventricle, 1-2 mm below the leaflets of the mitral valve. This image was acquired ECG and respiratory gated, using a bright blood 
Figure 2: Algorithms and formula's for calculation of $L V$ volumes with a full volume MR imaging data set of the complete left ventricle or geometrical models based on a few MRI slices.

Full volume data set (FV) $\mathrm{LVV}=\mathrm{S}_{1}+\mathrm{S}_{2}+\mathrm{S}_{3}+\ldots$

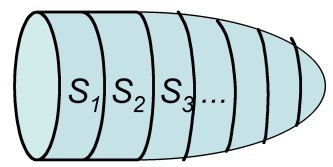

Biplane Ellipsoid model (BP)

$\mathrm{LVV}=\pi / 6 \times L \times\left(4 / \pi \times A_{m} / D\right) \times\left(4 / \pi \times A_{i} / L\right)$

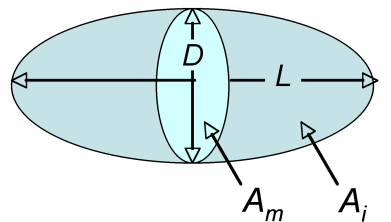

Modified Simpson rule model (SR)

$\mathrm{LVV}=A_{m} \times L / 3+\left(A_{m}+A_{p}\right) / 2 \times L / 3+1 / 3 \times A_{p} \times L / 3$

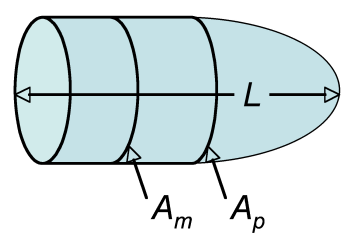

Hemisphere cylinder model (HC)

$\mathrm{LVV}=A_{m} \times L / 2+2 / 3 \times A_{m} \times L / 2=5 / 6 \times A_{m} \times L$

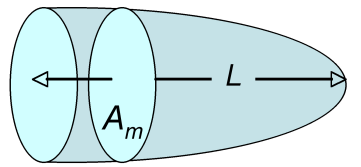

Single-plane Ellipsoid model (SP) $\operatorname{LVV}=\left(8 \times A_{i} \times A_{i}\right) /(3 \pi \times L)=0.85 \times\left(A_{i} \times A_{i}\right) / L$

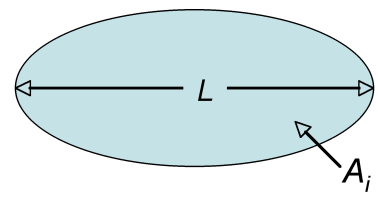

Modified Teichholz Formula (TF) $\mathrm{LVV}=[7 /(2.4+D)] \times D \times D \times D$

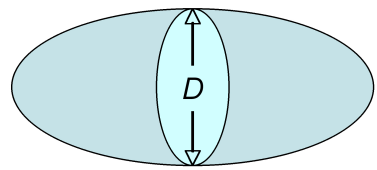

In the figure above the algorithms for full volume data set and the various geometrical models for the determination of LV volumes and EF are presented. $A_{i}=$ cross-sectional area of LV cavity in the LA plane, $A_{m}=$ cross-sectional area of the LV cavity in the SA plane, approximately 1-2 mm below the mitral valve, $A_{p}=$ cross-sectional area of the LV cavity in the SA plane, approximately at the base of the papillary muscles, $D=$ diameter of the LV cavity in the short axis plane, approximately 1-2 $\mathrm{mm}$ below the mitral valve, $L=$ longest length of LV cavity in the LA plane, LVV $=L V$ volumes (EDV and ESV, resp.), S1, S2, S3, .. = $1 \mathrm{~mm}$ SA slices from the apex to the base of the left ventricle.

gradient echo sequence with the following parameters for the mice studied: repetition time $11.6 \mathrm{~ms}$, echo time $2.5 \mathrm{~ms}$, flip angle 50 , 1 slice, $1 \mathrm{~mm}$ thickness, 8 signal averages, FOV $25.6 \times 25.6 \mathrm{~mm}$, matrix-size $164 \times 164$, resulting in an in-plane resolution $0.16 \times 0.16 \mathrm{~mm}^{2}$, acquisition time approximately 4 minutes. The SA images of the rats were acquired with the following parameters: repetition time $7 \mathrm{~ms}$, echo time $2.8 \mathrm{~ms}$, flip angle $50^{\circ}, 1$ slice, 1.263 $\mathrm{mm}$ thickness, 1 signal average, FOV 50x50mm, matrix-size 192x192, resulting in an in-plane resolution $0.26 \times 0.26 \mathrm{~mm}^{2}$, acquisition time approximately 5 minutes. For image analysis of the complete left ventricular volumes, 5-9 additional slices were planned parallel to the short axis slice with a slice thickness of $1 \mathrm{~mm}$ in the mice and $1.2 \mathrm{~mm}$ in the rats, without 
gap between slices. The slices covered the whole left ventricle, from the apex to the base. The images were acquired using the same pulse sequence. The average time for acquiring the images needed to assess EF with a full volume data, using 8 slices, is about 50 minutes.

\section{Image analysis}

All images were analyzed in Osirix (Dicom viewer, version 3.5, Pixmeo, Geneva, Switzerland). The end-diastolic volumes (EDV) and end-systolic volumes (ESV) of the left ventricle were considered the largest and the smallest areas, respectively, of the LV cavity in each slice. For the analysis of cine-MR images, the window width and level were manually adjusted to recognize the internal ventricular morphologic characteristics. For the measurements of LV volumes, the whole LV cavity was selected with semi-automated segmentation parameters in OsiriX. The papillary muscle was excluded from the left ventricular volume during analysis. The diameter $(D)$ of the LV volume on the short axis was measured as the longest distance between the septum and the ventricular wall. Length $(L)$ of the LV on the LA view was defined as the longest distance from the apex to the valves. On average, the time needed to analyze one slice was 3 minutes.

\section{Data analysis}

In all animals, end diastolic volume (EDV), end systolic volume (ESV), stroke volume (SV = EDV - ESV), and ejection fraction (EF = SV/EDV) were calculated from the end-diastolic and end-systolic volumes based on either the multi-slice short axis cine-images of the complete left ventricle (gold standard) or based on the different geometrical models as described by Dulce et al. $1993^{21}$. The algorithms and required imaging slices for these models can be found in figure 2.

\section{Statistical analysis}

Measurements of LV volumes and EF from cine MR imaging in both the wild type animals and the transgenic ATGL-/- mice are presented as mean values \pm standard deviation (SD). The correlations of LV volumes and EF, as measured with the various geometric models versus the full volume data set of the left ventricle (gold standard), were assessed by linear regression analysis. $\mathrm{R}^{2}, p$-values and $95 \%$ individual confidence intervals (also known as prediction intervals) are reported for all regression analysis. Differences in LV volumes and EF between the geometric models and the gold standard were analyzed for statistical significance with a 1-way ANOVA. Also differences in outcome measures between the different genotypes were assessed with a 1-way ANOVA for each model separately. $P$-value 
$<0.05$ was considered statistically significant. Reproducibility was tested by calculating the interclass correlation for two consecutive measurements for all geometrical models and the gold standard in 5 wild type mice. For measuring the two consecutive measurements, mice were taken out of the scanner in-between the measurements, where after they were repositioned and all preparatory steps (including shimming and acquisition of scout-images) were repeated. 


\section{Results}

\section{Reproducibility}

The interclass correlation coefficients (ICCS) for each model for the left ventricular volumes and ejection fraction are summarized in Table 1 . The ICC was $>0.85$ for all parameters in each model indicating a good reproducibility for all models.

Table 1: Interclass correlation coefficients of $L V$ volumes and ejection fraction in wild type animals

\begin{tabular}{|c|c|c|c|c|}
\hline$(n=5)$ & EDV (\%) & ESV (\%) & SV (\%) & EF (\%) \\
\hline FV & 0.944 & 0.915 & 0.981 & 0.995 \\
\hline BP & 0.991 & 0.994 & 0.986 & 0.975 \\
\hline SR & 0.995 & 0.981 & 0.986 & 0.955 \\
\hline $\mathrm{HC}$ & 0.979 & 0.997 & 0.961 & 0.996 \\
\hline SP & 0.997 & 0.989 & 0.976 & 0.939 \\
\hline $\mathrm{TF}$ & 0.983 & 0.893 & 0.887 & 0.866 \\
\hline
\end{tabular}

Presented above are the interclass correlation coefficients of a repeated measurement in 5 mice. Values were obtained with geometric models and a complete imaging set of the left ventricle acquired with cine-MRI. FV = full volume data set, $\mathrm{BP}=$ biplane ellipsoid model, $\mathrm{SR}=$ modified Simpson rule model, $\mathrm{HC}=$ hemisphere cylinder model, $\mathrm{SP}=$ single plane ellipsoid model, $\mathrm{TF}=$ modified Teichholz formula model .

\section{Validation of LV volumes and EF}

The values for LV volumes and EF in the various animal groups are summarized in Table 2. Only the modified Simpson Rule model (SR) gave statistically indifferent values for both the LV volumes and the EF compared to the Full Volume (FV) data set for all study groups. All models could pick up the marked differences in LV volumes and EF found between wild type and transgenic mice ( $p<0.05$, one-way ANOVA test, data not shown).

\section{Linear regression analysis}

The scatter plots with individual regression lines of the EF quantified by the models versus the gold standard (quantification based on FV data set) are provided in Figure 3. Pearson's 
correlation coefficients $(r)$, individual $95 \%$ confidence intervals $(\mathrm{Cl})$ and $\mathrm{R}^{2}$ can also be found in this figure. As the modified Simpson rule model was the only model that provided the correct absolute values for EF and LV volumes, we also present the scatter plots of the LV volumes and stroke volume with the full volume data set for this model in figure 4 (for mice and rats the data are presented separately).

Although all correlations were significant, only the hemisphere cylinder $(\mathrm{HC})$ model, the modified Simpson rule (SR) model and the biplane ellipsoid (BP) model showed a linear regression with a Pearson's correlation coefficient for EF $>0.85$. The other two models showed a poor correlation with correlation coefficients $<0.7$ and $R^{2}$-values $<0.45$. The $95 \%$ confidence interval was smallest for the modified Simpson rule model, as the $\mathrm{R}^{2}$ and $r$-values were the highest for this model $\left(R^{2}=0.937, r=0.968\right.$ and $\left.p<0.001\right)$. The $L V$ volumes and stroke volume, as calculated with the modified Simpson rule model, correlated with the full volume data set both in the mouse models as well as in the rat model. Although, it should be noticed that the correlations were stronger rat model. The $\mathrm{R}^{2}$-values ranged between 0.59-0.95 with $p$-values smaller than 0.01 for all correlations (also see figure 4). 
Table 2: Mean and Standard Deviation of LV volumes and Ejection Fraction

\begin{tabular}{|c|c|c|c|c|c|c|c|c|c|c|c|c|c|c|c|c|}
\hline \multicolumn{17}{|c|}{ Wild Type mice $(n=12)$} \\
\hline \multicolumn{5}{|c|}{$\operatorname{EDV}(\mu \mathrm{l})$} & \multicolumn{4}{|c|}{ ESV $(\mu l)$} & \multicolumn{4}{|c|}{ SV $(\mu \mathrm{l})$} & \multicolumn{4}{|c|}{$\mathrm{EF}(\%)$} \\
\hline FV & 52.7 & & 3.3 & & 14.8 & & 1.0 & & 37.9 & & 2.8 & & 71.5 & & 1.5 & \\
\hline $\mathrm{BP}$ & 84.8 & & 6.0 & $*$ & 18.0 & & 1.8 & NS & 66.8 & & 4.6 & $*$ & 79.0 & \pm & 1.1 & $*$ \\
\hline SR & 60.3 & & 2.7 & NS & 14.7 & & 1.4 & NS & 45.6 & & 2.1 & NS & 75.9 & \pm & 1.8 & NS \\
\hline $\mathrm{HC}$ & 71.2 & & 3.6 & $\S$ & 14.3 & & 1.4 & NS & 56.9 & & 2.7 & $*$ & 80.2 & \pm & 1.3 & $*$ \\
\hline $\mathrm{SP}$ & 58.0 & \pm & 5.0 & NS & 22.2 & \pm & 2.2 & NS & 35.9 & \pm & 3.7 & NS & 61.4 & \pm & 2.6 & $*$ \\
\hline $\mathrm{TF}$ & 149.5 & \pm & 15.5 & $*$ & 45.4 & & 7.5 & $*$ & 104.1 & \pm & 9.6 & $*$ & 71.1 & \pm & 3.0 & NS \\
\hline \multicolumn{17}{|c|}{ Transgenic mice $(n=12)$} \\
\hline & \multicolumn{4}{|c|}{$\operatorname{EDV}(\mu \mathrm{l})$} & \multicolumn{4}{|c|}{$\operatorname{ESV}(\mu \mathrm{l})$} & \multicolumn{4}{|c|}{$\operatorname{SV}(\mu \mathrm{l})$} & \multicolumn{3}{|c|}{$\mathrm{EF}(\%)$} & \\
\hline FV & 53.4 & & 2.7 & & 28.6 & & 3.3 & & 20.6 & & 3.8 & & 47.6 & & 4.2 & \\
\hline $\mathrm{BP}$ & 89.3 & & 4.9 & $*$ & 41.0 & & 5.8 & NS & 48.3 & \pm & 3.3 & $*$ & 55.9 & \pm & 4.9 & NS \\
\hline$S R$ & 55.0 & & 4.8 & NS & 27.1 & & 4.7 & NS & 27.9 & \pm & 1.6 & NS & 53.4 & \pm & 4.8 & NS \\
\hline $\mathrm{HC}$ & 74.2 & & 5.1 & NS & 35.2 & \pm & 4.9 & NS & 39.1 & \pm & 2.2 & $*$ & 54.7 & \pm & 4.2 & NS \\
\hline $\mathrm{SP}$ & 63.8 & & 5.7 & NS & 48.0 & \pm & 7.0 & NS & 15.8 & \pm & 2.1 & NS & 28.6 & \pm & 5.2 & $*$ \\
\hline $\mathrm{TF}$ & 180.8 & \pm & 20.3 & $*$ & 135 & \pm & 18.6 & $*$ & 45.8 & \pm & 9.6 & $*$ & 26.8 & \pm & 4.7 & $*$ \\
\hline \multicolumn{17}{|c|}{ Rat ischemic heart model $(n=11)$} \\
\hline & \multicolumn{4}{|c|}{$\operatorname{EDV}(\mathrm{ml})$} & \multicolumn{4}{|c|}{ ESV (ml) } & \multicolumn{4}{|c|}{$\mathrm{SV}(\mathrm{ml})$} & \multicolumn{3}{|c|}{$\mathrm{EF}(\%)$} & \\
\hline FV & 0.36 & \pm & 0.05 & & 0.23 & \pm & 0.04 & & 0.13 & \pm & 0.02 & & 35.2 & \pm & 5.7 & \\
\hline $\mathrm{BP}$ & 0.36 & \pm & 0.07 & NS & 0.24 & \pm & 0.06 & NS & 0.12 & \pm & 0.04 & NS & 33.6 & \pm & 11.9 & NS \\
\hline SR & 0.36 & \pm & 0.05 & NS & 0.23 & \pm & 0.04 & NS & 0.13 & \pm & 0.03 & NS & 36.1 & \pm & 6.4 & NS \\
\hline $\mathrm{HC}$ & 0.33 & \pm & 0.05 & $\S$ & 0.21 & \pm & 0.05 & NS & 0.11 & \pm & 0.03 & NS & 35.4 & \pm & 7.8 & NS \\
\hline $\mathrm{SP}$ & 0.24 & \pm & 0.11 & $*$ & 0.17 & \pm & 0.11 & $*$ & 0.07 & \pm & 0.03 & $*$ & 34.7 & \pm & 18.4 & NS \\
\hline TF & 0.39 & \pm & 0.29 & NS & 0.23 & \pm & 0.17 & NS & 0.16 & \pm & 0.16 & NS & 41.3 & \pm & 23.6 & $\S$ \\
\hline
\end{tabular}

Presented above are the averages and standard deviations of the LV volumes and EF \pm SD per group (WT mice, ATGL-/- mice and rats with cardiac ischemia) and per geometrical model. Values were obtained with geometric models and the complete imaging set of the entire left ventricle acquired with cine MRI. FV = full volume data set, $\mathrm{BP}=$ biplane ellipsoid model, $\mathrm{SR}=$ modified Simpson rule model, $\mathrm{HC}=$ hemisphere cylinder model, $\mathrm{SP}=$ single plane ellipsoid model, TF $=$ modified Teichholz formula model. ${ }^{*}=\mathrm{P}<0.05, \S=\mathrm{P}<0.10, \mathrm{NS}=$ not significant, when compared to the gold standard (FV). 
Figure 3: Scatter plots of ejection fractions of the various models vs. full volume data set (FV)
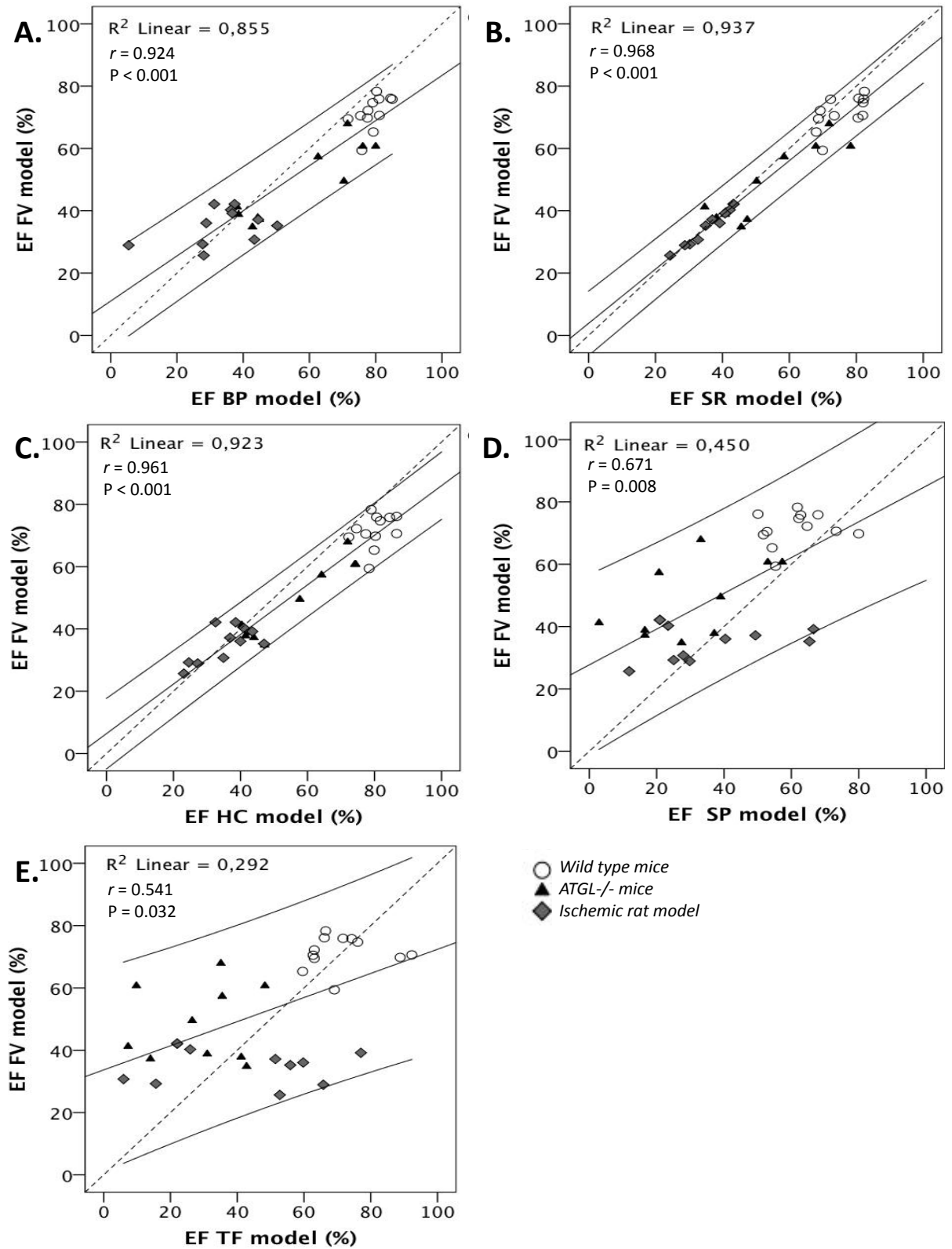

A. the biplane ellipsoid model, B. the modified Simpson rule model, C. the hemisphere cylinder model, D the single plane ellipsoid model and E. the modified Teichholz Formula. In all scatter plots the regression line is given with a 95\% confidence interval. R-squares and Pearson correlation coefficients (with according $p$-values) can also be found in the graph for each fit. 
Figure 4: Scatter plots of LV volumes and stroke volume for the modified Simpson rule model (SR) vs. full volume data set (FV)
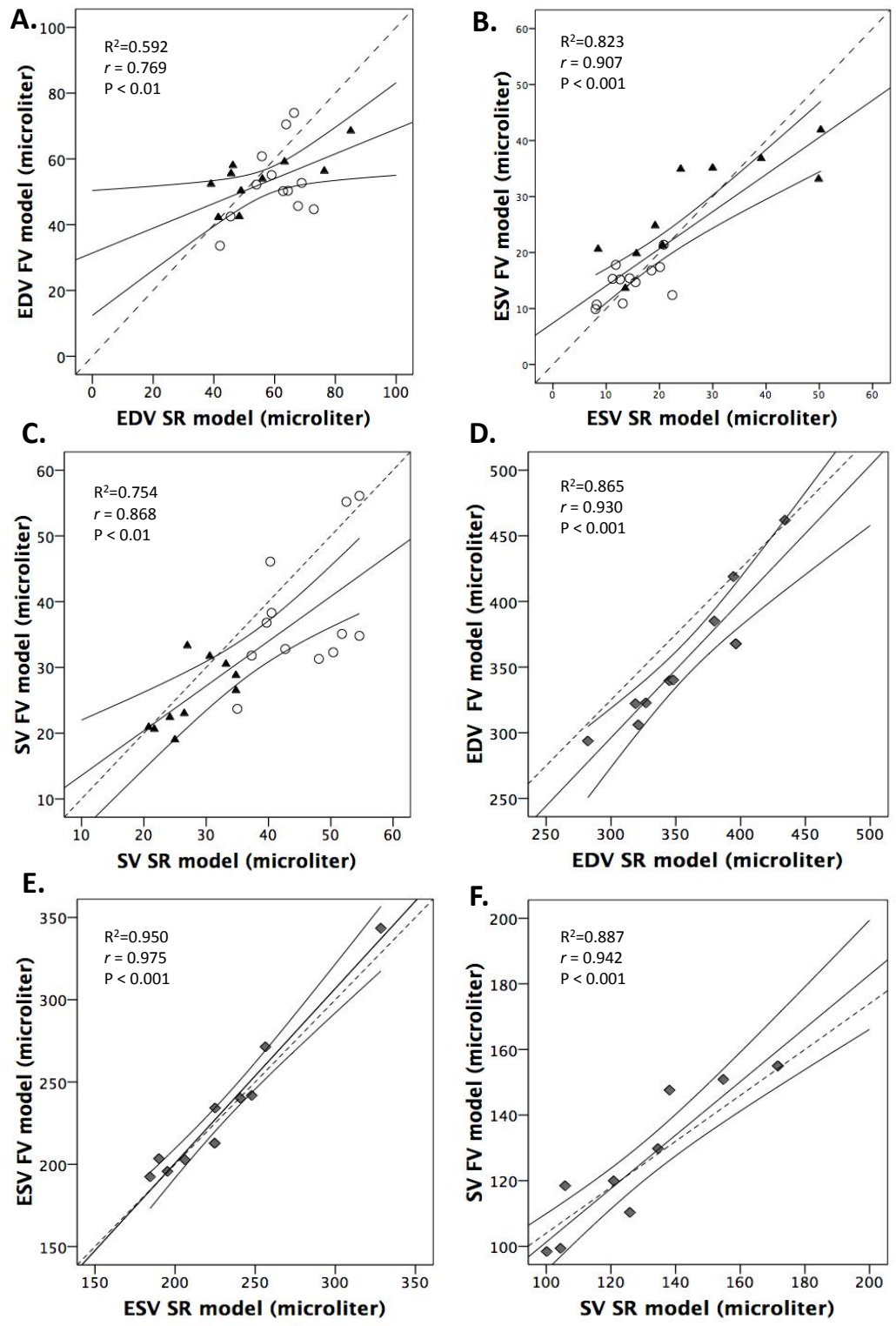

A. scatter plot for the end-diastolic volumes in WT and ATGL-/- mice, B. scatter plot for the end-systolic volumes in WT and ATGL-/- mice, C. scatter plot for the stroke volumes in WT and ATGL-/- mice, D. scatter plot for the end-diastolic volumes in rats with MI, E. scatter plot for the end-systolic volumes in rats with $\mathrm{MI}$ and F. scatter plot for the stroke volumes in rats with MI. In all scatter plots the regression line is given with a $95 \%$ confidence interval. The dotted line in the graph represents $x=y$. R-squares and Pearson correlation coefficients (with according p-values) can also be found in the graph for each fit. 


\section{Discussion}

In this study, we aimed to assess the validity of common geometrical models compared to the gold standard, a full volume data set, for quantification of LV volumes and EF with cineMRI in rodents. We showed that the reproducibility of all models was good, with higher variations in the Teichholz formula model. When the models were compared to the full volume data set, only the modified Simpson rule model showed no significant differences with the gold standard for all the calculated parameters. The values found for the LV volumes, stroke volume, and EF with the modified Simpson rule model correlated well with the full volume data set (gold standard). Also the EF found with the hemisphere cylinder and biplane ellipsoid model showed high correlation coefficients with the full volume data set. The two remaining models were poor predictors of EF.

Although these findings are new for MRI applications, previous research using other imaging modalities already pointed in this direction. For instance, it has been shown that the Teichholz formula model does not give comparable results in MRI compared to ultrasound ${ }^{19}$. These results also did not correlate with values found with the modified Simpson rule model in this study either ${ }^{19}$. The discrepancy in absolute values compared to the gold standard for all models except the modified Simpson rule, points out that care should be taken when cardiac function is compared between studies using different methodology.

By using the modified Simpson rule, one needs to acquire and assess only 3 imaging planes, thereby reducing total examination time by $10-45$ minutes compared to total examination time including positioning of the animal of 30-60 minutes in the present study. In addition, segmentation time is reduced by 2-3 fold (depending on the animal species used). The modified Simpson rule model is the only model that divides the heart into 3 different compartments, based on three imaging planes. This may explain why it is more accurate in estimating the LV volumes, and therefore it might also be more sensitive for non-symmetrical pathologies (like myocardial infarction, for instance). The other models are based on only one (modified Teichholz formule and single plane ellipsoid model) or two (Hemisphere and biplane ellipsoid models) imaging planes. The most simplified models, using only one plane, showed indeed the weakest correlations, whereas the two plane models did better. Although the EF as determined by these two plane models correlated well with the EF calculated from the full volume data set, they significantly over or underestimated the real volumes of the left ventricle. Therefore, only the modified Simpson rule can be applied in rodent studies of cardiac disease.

For high throughput studies, parallel imaging or simultaneous scanning of multiple animals might by used as an alternative to reduce scan time. These methods will, however, not lead to a reduction in segmentation time. Scanning multiple animals simultaneously is still a quite 
new technique, which requires specific system requirements ${ }^{25-27}$. Parallel imaging leads to a reduction of the signal-to-noise ratio and therefore may not always be appropriate ${ }^{28-31}$. The Simpson rule model could be a good alternative, and combining parallel imaging with the Simpson rule model will even further increase the throughput.

One of the limitations of this study is that the mouse model of transient cardiac disease, namely the ATGL-/- as a model for cardiomyopathy, is quite a rare model. Nonetheless, we choose to use this model as their cardiac function is severely impaired and the progression of their disease is rather extreme ${ }^{22}$, which introduces variation in the main outcome parameters as can be found in table 2 . This variation is not due to a poor reproducibility in this specific group, rather it is due to variation of the disease stage as cardiac function drastically drops with age. A greater variation in the outcome parameters gives a larger spread in the data and makes these data more suited for linear regression analysis. Therefore this model was ideal for looking at correlations in EF. In addition to the ATGL-/- mice, we also included WT mice and rats with myocardial infarction in the present study, since these are more commonly used.

Introducing the rat model of myocardial infarction in this study not only introduced a more common model of cardiac disease, but also increased the number of animals for linear regression analysis of EF. One drawback might be that we did handle two different kinds of species in the same analysis. Therefore we analyzed the correlations between the modified Simpson rule model and full volume data set for the LV volumes and SV for the different animal species separately. The LV volumes seemed to correlate better in the rat model than in the mouse model. The differences in the correlations found, are most likely due to the better estimation of the LV volumes in the segmentation analysis of the rat hearts due to relatively higher resolution of the left ventricle (as the LV is quite a bit bigger in rats). Also the spread of the EDV in the rat hearts was greater, resulting in a better spread of the data suitable for regression analysis.

In conclusion, the modified Simpson rule is a good alternative to a full volume data set and reduces scan and post-processing time. Comparing the absolute values as determined by different geometrical models should be done with caution as they give variable results.

\section{Funding}

This work was supported by the Center for Translational Molecular Medicine and the Netherlands Heart Foundation, Dutch Diabetes Research Foundation and Dutch Kidney Foundation (PREDICCt). Also V.S. is supported by a VENI grant from the Netherlands Organisation for Scientific Research (NWO). 


\section{References}

1. Uemura K, Pisa Z. Recent trends in cardiovascular disease mortality in 27 industrialized countries. World Health Stat Q 1985;38:142-162.

2. Uemura K, Pisa Z. Trends in cardiovascular disease mortality in industrialized countries since 1950. World Health Stat Q 1988;41:155-178.

3. Russell JC, Proctor SD. Small animal models of cardiovascular disease: tools for the study of the roles of metabolic syndrome, dyslipidemia, and atherosclerosis. Cardiovasc Pathol 2006;15:318-330.

4. Hasenfuss G. Animal models of human cardiovascular disease, heart failure and hypertrophy. Cardiovasc Res 1998;39:60-76.

5. Schneider JE, Cassidy PJ, Lygate C, Tyler DJ, Wiesmann F, Grieve SM, et al. Fast, high-resolution in vivo cine magnetic resonance imaging in normal and failing mouse hearts on a vertical 11.7 T system. J Magn Reson Imaging 2003;18:691-701.

6. Ruff J, Wiesmann F, Hiller KH, Voll S, von Kienlin M, Bauer WR, et al. Magnetic resonance microimaging for noninvasive quantification of myocardial function and mass in the mouse. Magn Reson Med 1998;40:43-48.

7. Ross AJ, Yang Z, Berr SS, Gilson WD, Petersen WC, Oshinski JN, et al. Serial MRI evaluation of cardiac structure and function in mice after reperfused myocardial infarction. Magn Reson Med 2002;47:1158-1168.

8. $\quad$ Epstein FH. MR in mouse models of cardiac disease. NMR Biomed 2007;20:238-255.

9. Hiller KH, Waller C, Haase A, Jakob PM. Magnetic resonance of mouse models of cardiac disease. Handb Exp Pharmacol 2008:245-257.

10. Young AA, Barnes H, Davison D, Neubauer S, Schneider JE. Fast left ventricular mass and volume assessment in mice with three-dimensional guide-point modeling. J Magn Reson Imaging 2009;30:514-520.

11. Hanusch C, Hoeger S, Beck GC. Anaesthesia of small rodents during magnetic resonance imaging. Methods 2007;43:68-78.

12. Cottrell JE. We care, therefore we are: anesthesia-related morbidity and mortality: the 46th Rovenstine Lecture. Anesthesiology 2008;109:377-388.

13. Haemmerle G, Moustafa T, Woelkart G, Buttner S, Schmidt A, van de Weijer T, et al. ATGLmediated fat catabolism regulates cardiac mitochondrial function via PPAR-alpha and PGC-1. Nat Med;17:1076-1085.

14. Iltis I, Kober F, Dalmasso C, Cozzone PJ, Bernard M. Noninvasive characterization of myocardial blood flow in diabetic, hypertensive, and diabetic-hypertensive rats using spin-labeling MRI. Microcirculation 2005;12:607-614.

15. Oostendorp M, Douma K, Wagenaar A, Slenter JM, Hackeng TM, van Zandvoort MA, et al. Molecular magnetic resonance imaging of myocardial angiogenesis after acute myocardial infarction. Circulation 2010;121:775-783.

16. Heijman E, Aben JP, Penners C, Niessen P, Guillaume R, van Eys G, et al. Evaluation of manual and automatic segmentation of the mouse heart from CINE MR images. J Magn Reson Imaging 2008;27:86-93.

17. Shohet RV, Kisanuki YY, Zhao XS, Siddiquee Z, Franco F, Yanagisawa M. Mice with cardiomyocyte-specific disruption of the endothelin-1 gene are resistant to hyperthyroid cardiac hypertrophy. Proc Natl Acad Sci U S A 2004;101:2088-2093. 
18. Franco F, Dubois SK, Peshock RM, Shohet RV. Magnetic resonance imaging accurately estimates LV mass in a transgenic mouse model of cardiac hypertrophy. Am J Physiol 1998;274:H679-683.

19. Bunck AC, Engelen MA, Schnackenburg B, Furkert J, Bremer C, Heindel W, et al. Feasibility of functional cardiac MR imaging in mice using a clinical 3 Tesla whole body scanner. Invest Radiol 2009;44:749-756.

20. Wiesmann F, Frydrychowicz A, Rautenberg J, Illinger R, Rommel E, Haase A, et al. Analysis of right ventricular function in healthy mice and a murine model of heart failure by in vivo MRI. Am J Physiol Heart Circ Physiol 2002;283:H1065-1071.

21. Dulce MC, Mostbeck GH, Friese KK, Caputo GR, Higgins CB. Quantification of the left ventricular volumes and function with cine MR imaging: comparison of geometric models with three-dimensional data. Radiology 1993;188:371-376.

22. Haemmerle G, Lass A, Zimmermann R, Gorkiewicz G, Meyer C, Rozman J, et al. Defective lipolysis and altered energy metabolism in mice lacking adipose triglyceride lipase. Science 2006;312:734-737.

23. Kenis $\mathrm{H}$, Zandbergen HR, Hofstra L, Petrov AD, Dumont EA, Blankenberg FD, et al. Annexin A5 uptake in ischemic myocardium: demonstration of reversible phosphatidylserine externalization and feasibility of radionuclide imaging. J Nucl Med 2010;51:259-267.

24. Palojoki E, Saraste A, Eriksson A, Pulkki K, Kallajoki M, Voipio-Pulkki LM, et al. Cardiomyocyte apoptosis and ventricular remodeling after myocardial infarction in rats. Am J Physiol Heart Circ Physiol 2001;280:H2726-2731.

25. Bock NA, Konyer NB, Henkelman RM. Multiple-mouse MRI. Magn Reson Med 2003;49:158167.

26. Bock NA, Nieman BJ, Bishop JB, Mark Henkelman R. In vivo multiple-mouse MRI at 7 Tesla. Magn Reson Med 2005;54:1311-1316.

27. Bishop J, Feintuch A, Bock NA, Nieman B, Dazai J, Davidson L, et al. Retrospective gating for mouse cardiac MRI. Magn Reson Med 2006;55:472-477.

28. Roemer PB, Edelstein WA, Hayes CE, Souza SP, Mueller OM. The NMR phased array. Magn Reson Med 1990;16:192-225.

29. Jakob PM, Griswold MA, Edelman RR, Manning WJ, Sodickson DK. Accelerated cardiac imaging using the SMASH technique. J Cardiovasc Magn Reson 1999;1:153-157.

30. Pruessmann KP, Weiger M, Scheidegger MB, Boesiger P. SENSE: sensitivity encoding for fast MRI. Magn Reson Med 1999;42:952-962.

31. Sodickson DK. Tailored SMASH image reconstructions for robust in vivo parallel MR imaging. Magn Reson Med 2000;44:243-251. 

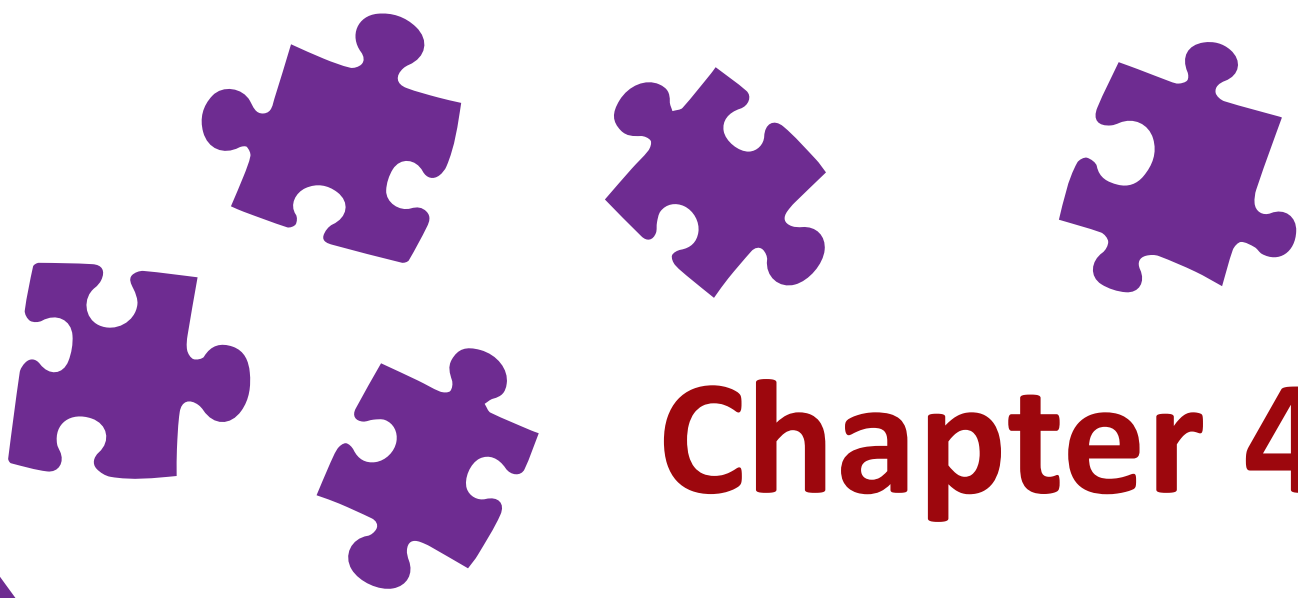

Chapter 4

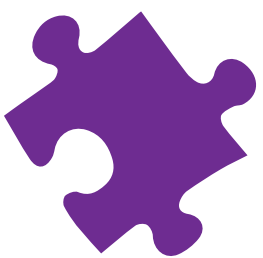

\section{ATGL-mediated fat catabolism regulates cardiac mitochondrial function via PPAR $\alpha$ and PGC- $1 \alpha$ and $\beta$}

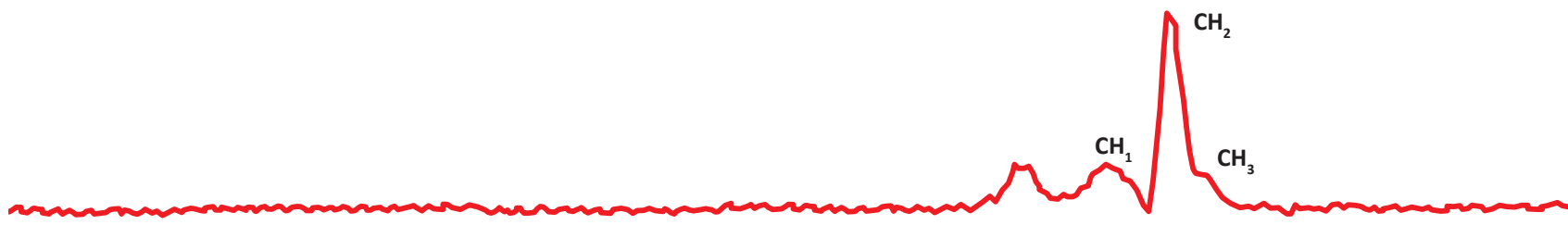

Guenter Haemmerle ${ }^{1}$, Tarek Moustafa ${ }^{2}$, Gerald Woelkart ${ }^{3}$, Sabrina Büttner ${ }^{1}$, Albrecht Schmidt ${ }^{4}$, Tineke van de Weijer ${ }^{5}$, Matthijs Hesselink ${ }^{6}$, Doris Jäger ${ }^{1}$, Petra Kienesberger ${ }^{1}$, Kathrin Zierler ${ }^{1}$, Renate Schreiber ${ }^{1}$, Thomas Eichmann ${ }^{1}$, Dagmar Kolb ${ }^{1}$, Petra Kotzbeck ${ }^{1}$, Martina Schweiger ${ }^{1}$, Manju Kumari ${ }^{1}$, Sandra Eder ${ }^{1}$, Gabriele Schoiswohl ${ }^{1}$, Nuttaporn Wongsiriroj ${ }^{1}$, Nina Pollak ${ }^{1}$, Franz P. W. Radner ${ }^{1}$, Karina Preiss-Landl ${ }^{1}$, Thomas Kolbe ${ }^{6}$, Thomas Rülicke ${ }^{7}$, Burkert Pieske ${ }^{4}$,

Michael Trauner ${ }^{2}$, Achim Lass ${ }^{1}$, Robert Zimmermann ${ }^{1}$, Gerald Hoefler $^{8}$, Saverio Cinti ${ }^{9}$, Erin E. Kershaw ${ }^{10}$, Patrick Schrauwen ${ }^{5}$, Frank Madeo ${ }^{1}$, Bernd Mayer ${ }^{3}$, and Rudolf Zechner ${ }^{1}$

${ }^{1}$ Institute of Molecular Biosciences, University of Graz, Austria, ${ }^{2}$ Laboratory of Experimental and Molecular Hepatology, Department of Internal Medicine, Medical University of Graz, Austria, ${ }^{3}$ Department of Pharmacology

and Toxicology, University of Graz, Austria, ${ }^{4}$ Department of Cardiology, Department of Internal Medicine, Medical University of Graz, Austria, ${ }^{5}$ Department of Human Biology, NUTRIM School for Nutrition, Toxicology and Metabolism, Maastricht, University Medical Centre, Maastricht, The Netherlands, ${ }^{6}$ Department of Human Movement Sciences, NUTRIM School for Nutrition, Toxicology and Metabolism, Maastricht University Medical Centre, Maastricht, The Netherlands, 'Biomodels Austria, Institute of Animal Breeding and Genetics, University of Veterinary Medicine, Vienna, Austria, ${ }^{8}$ Institute of Pathology, Medical University of Graz, Austria, ${ }^{9}$ Department of Molecular Pathology and Innovative Therapies, Faculty of Medicine, University of Ancona (Politecnica delle Marche), Italy, ${ }^{10}$ Division of Endocrinology and Metabolism, Department of Medicine, University of Pittsburgh,

Pittsburgh, PA, USA

Nature Medicine 17, 1076-1085 (2011), Received 20 April 2011, Accepted 08 July 2011, Published online 21

August 2011 


\section{Abstract}

Peroxisome proliferator--activated receptors (PPARs) are nuclear hormone receptors that regulate genes involved in energy metabolism and inflammation. For biological activity, PPARs require cognate lipid ligands, heterodimerization with retinoic $X$ receptors, and coactivation by PPAR- $\gamma$ coactivator- $1 \alpha$ or PPAR- $\gamma$ coactivator-1 1 (PGC- $1 \alpha$ or PGC-1 $\beta$, encoded by Ppargc1a and Ppargc1b, respectively). Here we show that lipolysis of cellular triglycerides by adipose triglyceride lipase (patatin-like phospholipase domain containing protein 2, encoded by Pnpla2; hereafter referred to as Atgl) generates essential mediator(s) involved in the generation of lipid ligands for PPAR activation. Atgl deficiency in mice decreases mRNA levels of PPAR- $\alpha$ and PPAR- $\delta$ target genes. In the heart, this leads to decreased PGC$1 \alpha$ and PGC-1 $\beta$ expression and severely disrupted mitochondrial substrate oxidation and respiration; this is followed by excessive lipid accumulation, cardiac insufficiency and lethal cardiomyopathy. Reconstituting normal PPAR target gene expression by pharmacological treatment of Atgl-deficient mice with PPAR- $\alpha$ agonists completely reverses the mitochondrial defects, restores normal heart function and prevents premature death. These findings reveal a potential treatment for the excessive cardiac lipid accumulation and often-lethal cardiomyopathy in people with neutral lipid storage disease, a disease marked by reduced or absent ATGL activity.

Note: supplemental materials of this paper are not published in this thesis, but can be found online via http://www.nature.com/nm/journal/v17/n9/full/nm.2439.html 


\section{Introduction}

PPARs belong to the superfamily of nuclear hormone receptors. Ligand-induced activation of PPARs controls the expression of innumerable genes involved in energy homeostasis, lipid and lipoprotein metabolism, and inflammation. The PPAR family consists of PPAR $\alpha$, PPAR $\delta$ (also named PPAR $\beta$ ), and three isoforms of PPAR $\gamma^{1}$. PPAR $\alpha$ is mainly found in oxidative tissues like cardiac muscle, skeletal muscle, and liver where it activates the transcription of genes stimulating fatty acid (FA) transport and oxidation, ketogenesis, and gluconeogenesis ${ }^{2}$. PPAR $\delta$ is expressed ubiquitously and activates genes for FA and glucose utilization ${ }^{3}$. PPAR $\gamma$ is more common in cells involved in fat storage and induces the expression of lipogenic genes ${ }^{4}$. Activation of all PPAR isoforms involves the binding of lipid ligands, dimerization with retinoic-X receptor (RXR), and co-activation by PGC-1 $\alpha$ or $\beta^{5-7}$. Since both PPAR $\alpha$ and PPAR $\delta$ are critical for the coordinated regulation of processes involved in the uptake, transport, and oxidation of energy substrates ${ }^{2,3}$, their dysregulation causes major metabolic derangements resulting in ectopic lipid accumulation in cardiac and skeletal muscle as well as in the liver. This lipid overflow in non-adipose tissues leads to pronounced "lipotoxicity" characterized by defective insulin signaling, insulin resistance, and cellular dysfunction eventually resulting in cell death.

Although it is currently not clear how many specific endogenous lipid ligands activate the PPARs and whether ligand preference varies in a tissue-specific manner, it is generally accepted that FA directly (as PPAR ligands) or indirectly (as precursors for other lipid ligands of PPARs) activate target gene expression. Established ligands include mono- and polyunsaturated FA, as well as FA derivatives such as FA-CoAs, eicosanoids, and phospholipids ${ }^{8-13}$. Cellular FA originate from the uptake of unesterified plasma FA, the uptake of FA generated by the lipoprotein lipase (LPL)-mediated hydrolysis of triglyceride-rich lipoproteins, or de novo synthesis. FA from all these sources can activate PPAR-mediated gene expression ${ }^{13,14}$; however, it is currently unknown whether FA require esterification to triglycerides (TG) and subsequent re-hydrolysis (lipolysis) before signal transduction to the nucleus. Lipolysis implies the activity of ATGL and/or hormone-sensitive lipase (HSL, official name LIPE) ${ }^{15}$. The relatively benign phenotype of $\mathrm{Hsl}$ deficiency in mice $(H s / K O)$ argues against a crucial role of this enzyme in the regulation of PPARs and energy homeostasis ${ }^{16,17}$. In contrast, deficiency of Atgl in mice (AtglKO synonymous for Pnpla2-/-) associates with reduced expression of oxidative phosphorylation (OXPHOS) genes ${ }^{18}$, massive lipid accumulation in multiple tissues, severe skeletal- and cardiomyopathy, and premature death ${ }^{19}$. Similar defects in lipid- and energy homeostasis are observed in humans with mutations in the ATGL gene ${ }^{20,21}$. All 
affected individuals suffer from neutral lipid storage disease (NLSD) and many patients develop severe skeletal- and cardiomyopathy that necessitates heart transplantation ${ }^{21}$.

These findings inspired the compelling hypothesis that provision of FA for PPAR activation in oxidative organs such as the heart requires ATGL-mediated lipolysis. To test this hypothesis, we assessed the expression of established PPAR $\alpha$ and $\delta$ target genes in relation to mitochondrial morphology, OXPHOS, and heart function in normal and mutant mice lacking either Hsl or Atgl. We show that Atgl-mediated lipolysis in cardiac muscle is essential for the biological activity of the PPAR $\alpha$-PGC-1 complex and heart function. 


\section{Methods}

\section{Animals}

AtglKO and HslKO mice were generated on a mixed genetic background (50\% C57BL/6 and 50\% 129/Ola) and backcrossed onto the C57BL/6 background strain for more than 10 generations $^{17,19}$. Experimental mice were obtained by breeding heterozygous AtgIKO males to heterozygous AtglKO females and heterozygous $\mathrm{Hs} / \mathrm{KO}$ males to heterozygous $\mathrm{Hs} / \mathrm{KO}$ females, respectively. Since AtgIKO mice develop premature morbidity and mortality, all studies were performed with mice less than 10 weeks of age. Conditional knockout mice lacking Atgl only in muscle were obtained by generating a mouse line with a loxP flanked Atgl gene (AtgIFLOX mice) and breeding these mice with transgenic mice expressing the Cre-recombinase under the control of the muscle-specific creatine kinase promoter ${ }^{54}$. Transgenic mice expressing murine Atg/ cDNA selectively in cardiac muscle were made using the cardiac-specific promoter from the $\alpha$-myosin heavy chain $(\alpha-\mathrm{MHC})$ gene. The pBS II SK+ vector containing the $\alpha-\mathrm{MHC}$ promoter region (Genbank: U71441) was kindly provided by Dr. Jeffrey Robbins (University of Cincinnati, OH, USA) ${ }^{55}$. Full-length Atg/ cDNA was amplified from mouse adipose tissue cDNA using the 5' AGG TCG ACA TGT TCC CGA GGG AGA CCA AGT GGA A 3' forward and 5' AGG TCG ACT CAG CAA GGC GGG AGG CCA GGT GGA T 3' reverse primers. The amplified Atgl cDNA was fused to the $\alpha-M H C$ promoter at the Sall site of the vector. For efficient expression of the transgene, a non-translated region of the human 6-globin gene (Genbank: U01317, position 62613 - 64297) was inserted into the HindIII site downstream to the Atgl cDNA. The final construct was digested with Notl to remove the vector sequence. After purification, the transgene was injected into the pronucleus of B6D2 zygotes and transferred to pseudopregnant females. PCR analysis using genomic DNA isolated from tail tips was performed to identify offspring carrying the Atgl transgene. Two founder lines were established showing elevated expression of the Atgl transgene. One of them was then bred to mice carrying the knockout allele to generate mice lacking Atgl in all tissues but expressing the Atg/ transgene exclusively in cardiac muscle (AtglKO-cmAtg/TG).

Mice were kept on a regular chow diet and allowed ad libitum access to food and water except when stated otherwise. PPAR agonist were administered to mice as follows: 1) the PPAR $\alpha$ agonist Wy14643 (Cayman Chemical Company, Ann Arbor, MI, USA) was provided via ad libitum feeding chow diet containing $0.1 \%$ Wy 14643 for the times indicated; 2 ) the PPAR $\alpha$ agonist fenofibrate (Sigma-Aldrich, St. Louis, MO, USA) was provided via ad libitum feeding chow diet containing $0.2 \%$ fenofibrate for 10 weeks; 3 ) the PPAR $\delta$ agonist GW501516 (Cayman Chemical Company) was administered by either intraperitoneal injection or by oral gavage at a dose of $5 \mu \mathrm{g}$ per g bodyweight. For intraperitoneal injection the agonist was dissolved 
in DMSO and diluted with $0.9 \% \mathrm{NaCl}$. For oral gavage, GW501516 (5 $\mu \mathrm{g} / \mathrm{g}$ bodyweight) was dissolved in DMSO and diluted with $0.75 \%$ cellulose carrier solution and administered semi-daily at 8 a.m. and 4 p.m., respectively; 4) the PPAR $\delta$ agonist GW0742 was provided via ad libitum feeding chow diet containing $0.01 \%$ GW0742. For fasting experiments, food was removed for $12 \mathrm{~h}$. All animal procedures were performed according to the guidelines of the Federation of European Laboratory Animal Science and were approved by Austrian government authorities.

\section{Quantitative RT-qPCR-based gene expression}

Total RNA was extracted with TRIzol reagent (Invitrogen, Carlsbad, CA, USA) and treated with DNasel. For first-strand cDNA synthesis, $1.5 \mu \mathrm{g}$ of total RNA was reverse transcribed at $42^{\circ} \mathrm{C}$ for $1 \mathrm{~h}$ using random hexamer primer (Applied Biosystems, Foster City, CA, USA) and Superscript $I^{\circ}$ reverse transcriptase (Invitrogen). Primers used for RT-qPCR were designed using $A B I$ Primer Express and/or Primer3. Primers were designed to span exon-intron boundaries with an amplicon size of less than $150 \mathrm{bp}$ and BLASTed for specificity. RT-qPCR reactions $(20 \mu \mathrm{l})$ contained $12.5 \mathrm{ng}$ cDNA, $330 \mathrm{nM}$ of each primer and $10.5 \mu \mathrm{l}$ of SYBR Green Master mix (Applied Biosystems) and were carried out using ABI-PRISM 7700HT detection system (Perkin Elmer, Waltham, MA, USA). Relative mRNA levels were quantified using the comparative delta-Ct method, normalized to $18 \mathrm{~S}$ rRNA. Primer sequences are listed in Supplementary Table 4.

\section{Oxygen consumption}

Tissue oxygen uptake was determined in vitro as previously described ${ }^{56}$. Briefly, freshly isolated ventricles were cut into slices of about 0.3-0.5 mm width using a Mcllwain mechanical tissue chopper (Mickle Laboratory Engineering, Gomshall, UK). Approximately 40 mg (wet weight) of heart slices were equilibrated at $37{ }^{\circ} \mathrm{C}$ in air-saturated Krebs solution (118 mM $\mathrm{NaCl}, 4.8 \mathrm{mM} \mathrm{KCl}, 1.2 \mathrm{mM} \mathrm{KH}_{2} \mathrm{PO}_{4}, 1.5 \mathrm{mM} \mathrm{CaCl}_{2}, 25 \mathrm{mM} \mathrm{NaHCO}_{3}, 1.2 \mathrm{mM} \mathrm{MgCl}_{2}$ ) containing $10 \mathrm{mM}$ glucose or $1.2 \mathrm{mM}$ palmitate bound to $10 \%$ BSA buffered with $10 \mathrm{mM} \mathrm{HEPES}(\mathrm{pH} 7.4)$ for $5 \mathrm{~min}$ and then transferred to an air-tight bottle containing $1.8 \mathrm{ml}$ of air-saturated stirred Krebs buffer. Consumption of oxygen was measured with a Clarke-type $\mathrm{O}_{2}$ electrode (ISO 2 World Precision Instruments, Mauer, Germany) and continuously recorded via a PowerLab system (ADInstruments, Hastings, UK) connected to the $\mathrm{O}_{2}$ electrode. After recording basal oxygen uptake over $10 \mathrm{~min}$, succinate (1 mM final concentration), a substrate for complex II, and sodium cyanide (1 mM), an inhibitor of complex IV of the electron transport chain, were injected in succession through a rubber septum to confirm that the changes in oxygen uptake were due to mitochondrial respiration. The typical observation time for each effector 
was 3 min. Oxygen uptake was calculated as the rate of decrease in $\mathrm{O}_{2}$ concentration after the addition of muscle segments. The electrode was calibrated daily.

\section{Preparation of intramyofibrillar and sacrolemmal mitochondria}

Mice were sedated and sacrificed by decapitation. The heart was rapidly ( $<30 \mathrm{~s}$ ) excised and placed into ice-cold isolation-medium containing $300 \mathrm{mM}$ sucrose, $20 \mathrm{mM} \mathrm{K}$-TES and $0.2 \mathrm{mM}$ EGTA ( $\mathrm{pH} 7.2$; with $\mathrm{KOH}$ ). The cardiac tissue was immediately processed for mitochondrial isolation. Briefly, tissues were freed of adipose and extraventricular tissue, minced with pre-cooled scissors and homogenized in a Potter homogenizer. Tissue homogenates were centrifuged at $800 \mathrm{~g}$ for $10 \mathrm{~min}$ at $4^{\circ} \mathrm{C}$. This resulted in a pellet containing the intramyofibrillar mitochondria and a supernatant containing the subsarcolemmal mitochondria. These 2 fractions were handled separately according to the following protocol: (i) For the isolation of subsarcolemmal mitochondria, the supernatant was centrifuged at 9,000 x g for $10 \mathrm{~min}$ at $4^{\circ} \mathrm{C}$. The pellet was resuspended in isolation medium, recentrifuged at 9,000 $\mathrm{g}$ for $10 \mathrm{~min}$ at $4^{\circ} \mathrm{C}$, and again resuspended by hand-homogenization in a small glass homogenizer in 80 $\mu \mathrm{l}$ of isolation medium. (ii) The original pellet from the first centrifugation step with fibers and intramyofibrillar mitochondria was resuspended in $20 \mathrm{ml}$ isolation buffer in a Potter homogenizer. The homogenate was centrifuged at $800 \mathrm{xg}$ during $10 \mathrm{~min}$ at $4^{\circ} \mathrm{C}$. The pellet was resuspended in isolation buffer in a homogenizer in the presence of proteinase Nagarse (Fluka, Zwijndrecht, The Netherlands; 8 units per $\mathrm{g}$ of tissue). The tissue homogenate was centrifuged at $5,000 \times \mathrm{g}$ for $5 \mathrm{~min}$ at $4^{\circ} \mathrm{C}$ and the resulting pellet was again resuspended in isolation buffer and subsequently centrifuged at $800 \times \mathrm{g}$ for $10 \mathrm{~min}$ at $4^{\circ} \mathrm{C}$. Then, the supernatant was centrifuged at $9,000 \times \mathrm{g}$ for $10 \mathrm{~min}$ at $4^{\circ} \mathrm{C}$ and the pellet containing the intramycellular mitochondrial fraction was resuspended by hand-homogenization in a small glass homogenizer in $80 \mu$ of isolation medium. The protein concentrations of mitochondrial pellets were measured using fluorescamine (Fluram; Fluka) with BSA as standard. Freshly isolated mitochondria were used immediately for mitochondrial respirometry.

\section{Mitochondrial respirometry}

Mitochondrial oxygen consumption was measured using a two-chamber oxygraph (OROBOROS Instruments, Innsbruck, Austria). Freshly isolated cardiac mitochondria ( $0.1 \mathrm{mg}$ of mitochondrial protein for pyruvate and for carnitine plus palmitoyl-CoA) were incubated in $100 \mathrm{mM}$ sucrose, $20 \mathrm{mM} \mathrm{K}$-TES ( $\mathrm{pH} 7.2), 50 \mathrm{mM} \mathrm{KCl}, 2 \mathrm{mM} \mathrm{MgCl}, 1 \mathrm{mM}$ EDTA, $4 \mathrm{mM} \mathrm{KH}_{2} \mathrm{PO}_{4}, 3 \mathrm{mM}$ malate, and $0.1 \%$ of BSA. Substrates used to feed mitochondrial respiration were as follows: $5 \mathrm{mM}$ pyruvate and $10 \mathrm{mM}$ glutamate (glycolytic substrates), or $2 \mathrm{mM}$ carnitine and $50 \mu \mathrm{M}$ palmitoyl-CoA (FA substrate). After measuring ADP driven 
state 3 respiration by addition of $450 \mu \mathrm{M}$ ADP, oligomycin (in 96\% ethanol) was added to block mitochondrial F1-F0 ATPase to examine the residual respiration reflecting proton leak (state 4). Titration with $0.5 \mu \mathrm{M}$ additions of the chemical uncoupler carbonyl cyanide p-trifluoromethoxyphenylhydrazone (FCCP) (in 96\% ethanol) was used to measure maximal mitochondrial respiratory capacity (state uncoupled, state U). Pilot experiments showed that ethanol itself did not have any effects on the parameters measured. Traces were randomized.

\section{Determination of mitochondrial membrane potential, mitochondrial particle size, and non-esterified thiol concentrations}

Cardiac muscle mitochondria were isolated by a standard protocol. Protein concentrations of purified mitochondria were determined using the Bradford assay (Biorad, Hercules, CA, USA). For quantification of mitochondrial membrane potential, mitochondria were stained with tetramethylrhodamine-methylester-perchlorate (TMRM, Invitrogen), a fluorescent dye that is readily sequestered by active mitochondria dependent on the mitochondrial transmembrane potential. $12.5 \mu \mathrm{g}$ mitochondria were stained with $0.5 \mu \mathrm{g} /$ $\mathrm{ml}$ TMRM within buffer $\mathrm{A}$ (0.2 M sucrose, $5 \mathrm{mM}$ succinate, $10 \mathrm{mM}$ TRIS-MOPS, $1 \mathrm{mM} \mathrm{H}_{3} \mathrm{PO}_{4^{\prime}}$ $10 \mu \mathrm{M}$ EGTA, $2 \mu \mathrm{M}$ Rotenone; $\mathrm{pH} 7.4)^{57}$ in a final volume of $250 \mu \mathrm{l}$ for $15 \mathrm{~min}$ at $37^{\circ} \mathrm{C}$ in the dark. Relative fluorescence intensity (detected at $585 \mathrm{~nm}$ with $42 \mathrm{~nm}$ bandwidth) was quantified using flow cytometry FACS Aria equipped with a $488 \mathrm{~nm}$ laser (Becton Dickinson, Franklin Lakes, NJ, USA). Additionally, mitochondrial particle size was determined using forward scatter analysis of mitochondrial suspensions in buffer A. For each sample, 30,000 mitochondria were evaluated with BD FACS Diva software. For determination of oxidized proteins, free thiol groups were quantified using the Ellman/Cystamine method ${ }^{58}$. Mitochondria were suspended in RIPA buffer (25 mM TRIS- $\mathrm{HCl}$ pH 7.6, $150 \mathrm{mM} \mathrm{NaCl}, 1 \%$ NP-40, $1 \%$ sodiumdeoxycholate, $0.1 \%$ SDS) to a final concentration of $1 \mu \mathrm{g} / \mu \mathrm{l}$. $25 \mu \mathrm{l}$ of lysed mitochondria were added to $175 \mu \mathrm{RSH}$-buffer $\left(0.1 \mathrm{M} \mathrm{KH}_{2} \mathrm{PO}_{4} / \mathrm{Na}_{2} \mathrm{HPO}_{4}, \mathrm{pH}\right.$ 8.0) in 96-well plates and incubated with $50 \mu \mathrm{l}$ of reagent mix (DTNB 5,5'-dithio-bis-2-nitrobenzoic acid) and cystamine diluted 1:10 in RSH-buffer) for $10 \mathrm{~min}$ in the dark. Samples were measured at OD $412 \mathrm{~nm}$ using a GENiosPro plate reader (Tecan, Männedorf, Germany). For calculation of relative free thiol groups, sample blanks (mitochondrial suspension without reagent) as well as reagent blanks (no mitochondria added) were subtracted.

\section{Western blot analysis of mitochondrial proteins}

Mitochondrial proteins were separated by $12.5 \%$ SDS-PAGE followed by Western Blot analysis using PVDF membranes and CAPS buffer (10 mM 3-cyclohexylamino-1-propanesulfonic acid, 
$10 \%$ methanol) for protein transfer. Blots were probed with mouse monoclonal antibodies (Abcam, Cambridge, UK) against NADH dehydrogenase (ubiquinone) $1 \alpha$ subcomplex subunit 9 (NDUFA9, ab55521; 1:1,000), succinate dehydrogenase complex subunit A (SDHA, ab14715; 1:20,000), and mitochondrial cytochrome c oxidase 1 (MTCO1, ab14705; 1:2,000) and a peroxidase-conjugated affinity-purified secondary antibody obtained from Sigma. For quantification of relative expression levels blots were scanned using a densitometer (Molecular Dynamics, Model P.D. 300) and quantified with ImageQuant Version 5.1 (GE Healthcare, Chalfont St. Gilles, UK). MTCO1 signals detected in WT mitochondria were normalized to 1 and the relative expression in Atgl-deficient mitochondria were calculated accordingly.

\section{Fatty acid oxidation}

Fatty acid $\beta$-oxidation was measured as described by Nemoto et $a l .{ }^{59}$ with some modifications. Fresh tissues were homogenized in four volumes of $0.25 \mathrm{M}$ sucrose, $1 \mathrm{mM}$ EDTA, and $1 \mathrm{mM}$ Tris- $\mathrm{HCl}$ pH 7.4 using a potter homogenizer with teflon pestle. Crude homogenates were incubated with $200 \mu \mathrm{l}$ assay-buffer containing $150 \mathrm{mM} \mathrm{KCl}, 10 \mathrm{mM}$ HEPES, $0.1 \mathrm{mM}$ EDTA, 1 $\mathrm{mM}$ potassium phosphate buffer ( $\mathrm{pH}$ 7.2), $5 \mathrm{mM}$ malonate, $10 \mathrm{mM} \mathrm{MgCl}, 1 \mathrm{mM}$ carnitine, $0.15 \%$ BSA, 5 mM ATP plus $1 \mu \mathrm{Ci}\left[9,10-{ }^{3} \mathrm{H}(\mathrm{N})\right]$ palmitic acid (American Radiolabeled Chemicals, St. Louis, MO, USA) and $50 \mu \mathrm{M}$ unlabeled palmitic acid saponified with $20 \mathrm{mM} \mathrm{NaOH}$ and $1 \%$ BSA. The reaction was incubated for $30 \mathrm{~min}$ at $25^{\circ} \mathrm{C}$ and stopped by the addition of $200 \mu \mathrm{l} 0.6$ $\mathrm{N}$ perchloric acid. After centrifugation $(13,000 \mathrm{rpm}, 10 \mathrm{~min})$ supernatants were transferred to a new vial and non-catabolized FA were removed by extracting the samples twice with 1.6 $\mathrm{ml} \mathrm{n}$-hexane. Between extraction steps, the mixture was centrifuged at 5,000 rpm for $5 \mathrm{~min}$. The radioactivity associated with palmitate oxidation products was determined by liquid scintillation counting. Fatty acid $\beta$-oxidation rates were expressed as $\mathrm{cpm} / \mathrm{min} / \mathrm{mg}$ protein.

\section{Mouse echocardiography}

Echocardiographic analyses were performed under light isoflurane anaesthesia. Mice were allowed to breathe spontaneously. 2D guided M-mode echoes were obtained from short- and long-axis views at the level of the largest LV-diameter using a VS-VEVO 770 high resolution imaging system (Visualsonics, Toronto, Canada) equipped with a real-time microvisualization scanhead (30 MHz). LV end-diastolic (LVEDD) and end-systolic (LVESD) dimensions were measured from original tracings by using the leading edge convention of the American Society of Echocardiography. All measurements and calculations were done in 
triplicates. Percent fractional shortening (FS), ejection fraction (EF), and LV mass (LVM) were calculated as previously described ${ }^{60}$.

\section{Electron microscopy}

Mice were sacrificed in the non-fasted state at the age of 8-10 weeks with an overdose of anesthetic (xylazine-ketamine) and immediately perfused with $4 \%$ paraformaldehyde in $0.1 \mathrm{M}$ phosphate buffer, $\mathrm{pH}$ 7.4, for $2 \mathrm{~min}$. Cardiac muscle was dissected using a Zeiss OPI1 surgical microscope (Carl Zeiss, Oberkochen, Germany). Small tissue fragments were fixed in $2 \%$ glutaraldehyde and $2 \%$ paraformaldehyde in $0.1 \mathrm{M}$ phosphate buffer, $\mathrm{pH} 7.4$, for $4 \mathrm{~h}$, postfixed in 1\% osmium tetroxide, and embedded in an Epon-Resin (Hexion, Columbus, $\mathrm{OH}$, USA) mixture. Semi-thin sections $(2 \mu \mathrm{m})$ were stained with toluidine blue, and thin sections were obtained with an MT-X ultratome (RMC,Tucson, AZ, USA), stained with lead citrate, and examined with a Zeiss EM 902 transmission electron microscope (Carl Zeiss).

\section{Measurements of PPAR $\alpha$ and PPAR $\delta$ protein concentrations in cell nuclei.}

The Qproteome Nuclear Protein tissue kit (Qiagen, Hilden, Germany) was used for the preparation of nuclear proteins from heart and liver tissue according to the manufacturer's instructions. Nuclear protein concentrations of PPAR $\alpha$ and PPAR $\delta$ were analyzed by using a non-radioactive transcription factor assay kit (Cayman Chemical Company). The enzymelinked immunosorbent assay was performed as recommended by the manufacturer.

\section{PPAR $\alpha$ trans-activation assay and luciferase assay}

Human hepatoma HepG2 cells were seeded into 24-well plates and cultured in DMEM containing $10 \%$ FBS or in medium supplemented with $30 \mu \mathrm{M}$ linoleic acid complexed to BSA 5 days prior to transfection. Cells were transfected with the peroxisome proliferator response element (PPRE) -tk-luciferase reporter plasmid (containing 6 copies of the PPRE ligated to the herpes simplex thymidine kinase promoter upstream of the luciferase gene), the PSG5 expression vector containing the CDNA of human PPAR $\alpha$ and the HisMaxC expression vector encoding mouse Atgl cDNA using the TurboFect reagent (Fermentas, St. Leon-Rot, Germany). "Empty" pSG5 plasmid DNA was added when necessary to assure equal amounts of DNA in each transfection. A CMV-ß-galactosidase expression vector was co-transfected to normalize transfection efficiency. The expression plasmids for (PPRE) $)_{6}$-tkluciferase and human PPAR $\alpha$ were kindly provided by B. Staels, University of Lille, France. $4 \mathrm{~h}$ after transfection, the media was changed to DMEM containing $10 \%$ charcoal-stripped FBS (Sigma-Aldrich). Luciferase activities were measured $48 \mathrm{~h}$ post-transfection using a 
LumiStar luminometer (BMG LABTECH, Offenburg, Germany). ß-galactosidase activity in cell lysates was determined using 2-nitrophenyl-ß-D-galactopyranoside as substrate. Luciferase activities were normalized to ß-galactosidase activity and calculated relative to control transfections (cells transfected with the luciferase reporter, the CMV-ß-galactosidase and the pSG5 vector), which were set as $100 \%$.

\section{Tissue TG analysis}

Mice were anaesthetized and blood was removed from the left ventricle. Mice were then perfused with $0.9 \% \mathrm{NaCl}$. Tissues were excised, washed in phosphate-buffered saline, weighed, and frozen. Total lipids were extracted from various organs by standard techniques. Tissue lipids were dried in a stream of nitrogen and re-dissolved by brief sonication in $1 \%$ Triton-X100. TG content was measured with the TG (GPO) reagent (Thermo Electron, Noble Park, Australia).

\section{Measurement of cardiac muscle glycogen content}

Cardiac muscle of WT and AtgIKO mice were homogenized with an Ultra Turrax (IKA, Staufen, Germany) in $0.03 \mathrm{M} \mathrm{HCl}(20 \mu \mathrm{l} / \mathrm{mg}$ tissue) on ice. For the determination of total glucose concentration, aliquots of tissue lysates were mixed with equal volumes of $2 \mathrm{M} \mathrm{HCl}$ and incubated for $2 \mathrm{~h}$ at $90^{\circ} \mathrm{C}$. Lysates were then neutralized with $2 \mathrm{M} \mathrm{NaOH}$. To determine free glucose concentration, aliquots of cardiac muscle lysates were mixed with equal amounts of $2 \mathrm{M} \mathrm{HCl}$ and immediately neutralized with $2 \mathrm{M} \mathrm{NaOH}$. Glucose concentrations were measured using the D-Glucose-HK-Kit (Megazyme International Ireland, Wicklow, Ireland). The amount of cardiac glycogen was calculated from the difference of tissue total glucose and free glucose concentrations.

\section{Analysis of plasma parameters}

Blood samples were collected by retro-orbital puncture from isoflurane anesthetized mice. Plasma was obtained after centrifugation (15 $\mathrm{min}, 3,000 \mathrm{rpm}, 4^{\circ} \mathrm{C}$ ) and concentrations of TG and FA were determined using commercial kits (Thermo Electron; Wako Chemicals, Neuss, Germany).

\section{Tissue TG hydrolase assay}

Assays were performed as previously described19. In brief, tissues were surgically removed and washed in ice-cold PBS containing $2 \mathrm{lU} / \mathrm{ml}$ heparin and $1 \mathrm{mM}$ EDTA. Tissues were homogenized on ice in lysis buffer A (0.25 M sucrose, 1 mM EDTA, 1 mM dithiothreitol, 20 
$\mu \mathrm{g} / \mathrm{ml}$ leupeptin, $2 \mu \mathrm{g} / \mathrm{ml}$ antipain, $1 \mu \mathrm{g} / \mathrm{ml}$ pepstatin, $\mathrm{pH} 7.0$ ) using an UltraTurrax (IKA). The infranatants were obtained after centrifugation at $20,000 \times \mathrm{g}$, at $4^{\circ} \mathrm{C}$ for $90 \mathrm{~min}$. Triolein was used as substrate containing [9,10-3H]triolein (NEN Life Science Products, Boston, MA, USA) as radioactive tracer. The substrate was prepared by sonication (Virsonic 475, Virtis, Gardiner, NJ, USA) as described previously19. The incubation was performed at $37^{\circ} \mathrm{C}$ for $60 \mathrm{~min}$ with $0.1 \mathrm{ml}$ of substrate and $0.1 \mathrm{ml}$ infranatant. The reaction was terminated by adding $3.25 \mathrm{ml}$ of methanol/chloroform/heptane (10:9:7) and $1 \mathrm{ml}$ of $0.1 \mathrm{ml} 0.1 \mathrm{M}$ potassium carbonate, $0.1 \mathrm{M}$ boric acid, $\mathrm{pH}$ 10.5. After centrifugation ( $800 \times \mathrm{g}, 20 \mathrm{~min}, \mathrm{RT}$ ) the radioactivity in $1 \mathrm{ml}$ upper phase was determined by liquid scintillation.

\section{Assay of tissue Lipoprotein Lipase activity}

Hearts were surgically removed from the animals and put into ice-cold tubes containing 1 $\mathrm{ml}$ of Dulbecco's modified Eagle's medium with $2 \%$ BSA and $2 \mathrm{U} / \mathrm{ml}$ heparin. After the tissue was minced with scissors, it was incubated in medium for $1 \mathrm{~h}$ at $37^{\circ} \mathrm{C}$. Enzyme activities of LPL were assayed by standard methods.

\section{Relative quantification of mitochondrial DNA copy numbers}

Total DNA was isolated from hearts of 8 to10-week old female WT and AtglKO mice and treated with RNase A. The mitochondrial (mt) DNA content relative to nuclear DNA was performed by RT-qPCR using the ABI-StepOne Plus (Applied Biosystems). Relative mtDNA content was determined using the delta-Ct method. Primers of the mtDNA encoded CO1 gene were (fwd) 5-TGC TAG CCG CAG GCA TTA C-3; (rev) 5-GGG TGC CCA AAG AAT CAG AAC3 and primers of the single-copy nuclear gene Ndufv1 were (fwd) 5-CTT CCC CAC TGG CCT CAA G-3; (rev) 5-CCA AAA CCC AGT GAT CCA GC-3.

\section{Measuring animal oxygen consumption and calculating respiratory quotient (RQ)}

Mice were housed in a laboratory animal monitoring system (LabMaster, TSE Systems, Bad Homburg, Germany) that allows the continuous measurement of oxygen consumption and carbon dioxide elimination of the body for calculating RQs (the ratio of carbon dioxide elimination versus oxygen consumption). For fasting experiments, food was withdrawn in the morning and oxygen consumption and carbon dioxide production were monitored after $3 \mathrm{~h}$ for a period of $5 \mathrm{~h}$. 


\section{Statistical analysis}

Statistical significance was determined by the Student's unpaired two-tailed $t$-test. Group differences considered significant for $p<0.05(*), p<0.01(* *)$, and $p<0.001(* * *)$. 


\section{Results}

\section{ATGL deficiency impairs PPAR target gene expression}

Analysis of mRNA expression in cardiac muscle of AtgIKO mice revealed markedly reduced expression of PPAR $\alpha$ and $\delta$ target genes including acyl-CoA oxidase (AOX,-72\%), medium-chain acyl-CoA dehydrogenase (MCAD, -64\%), long-chain acyl-CoA dehydrogenase (LCAD, -67\%), very long-chain acyl-CoA dehydrogenase (VLCAD, -77\%), carnitine palmitoyltransferase-1b (CPT-1b, -75\%), and pyruvate dehydrogenase kinase-4 (PDHK-4, -79\%) compared to the levels found in wild-type (WT) mice (Fig. 1a). Reduction in the expression levels of these genes in HsIKO mice was less pronounced (MCAD, -42\%; VLCAD, -26\%; CPT-1b, -44\%) or nonexistent (AOX, LCAD, PDHK-4). Analysis of liver samples showed a similar mRNA expression profile characterized by marked reduction of all PPAR $\alpha$ and $\delta$ target genes examined (-50\% to $-90 \%$ ) in AtglKO mice (Fig. 1b), whereas only liver-specific fatty acid binding protein (L-FABP) expression was reduced in $\mathrm{Hs} / \mathrm{KO}$ mice (-58\%). Measurements of PPAR proteins in

Figure 1: Expression of PPAR $\alpha$ and $\delta$ target genes and PGC-1 $\alpha$ and $\beta$ in AtglKO, HsIKO, and WT tissues.
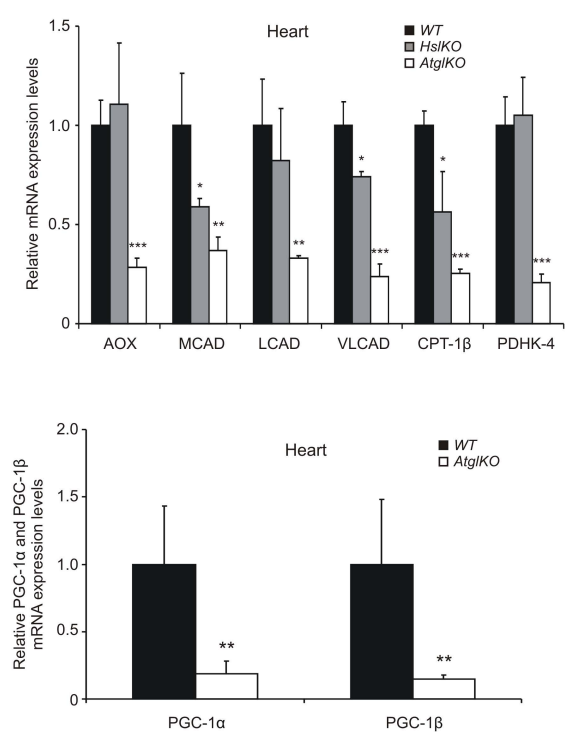

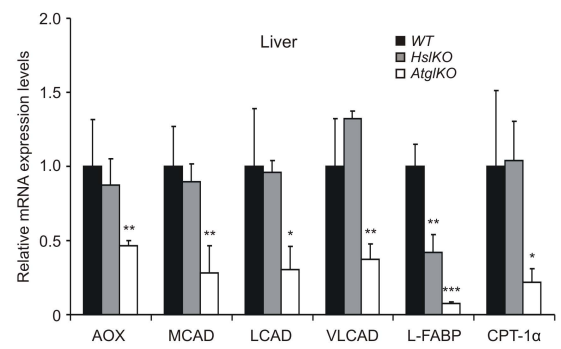

d

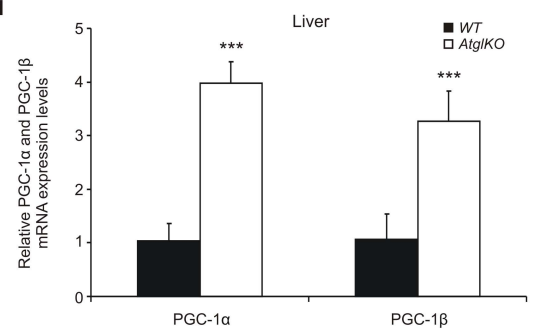

mRNA expression levels for selected PPAR $\alpha$ and $\delta$ target genes and PGC- $1 \alpha$ and $\beta$ were determined by RT-qPCR analysis. Cardiac (a) and hepatic (b) mRNA expression of PPAR $\alpha$ and $\delta$ target genes were markedly decreased in fasted 8 to 10 -week old female AtgIKO mice compared to age-matched Hs/KO and WT mice. PGC-1 $\alpha$ and PGC-1 $\beta$ mRNA levels were also reduced in cardiac muscle (c) but increased in the liver (d) of fasted AtglKO mice compared to $W T$ mice. $n=4$. All values are displayed as means + s.d. ${ }^{*} P<0.05, * * P<0.01$ and ${ }^{* * *} P<0.001$. 
AtgIKO mice revealed reduced concentrations of PPAR $\alpha$ in the heart (-37\%) and liver (-33\%) (Supplementary Fig. 1a), and PPAR $\delta$ in the heart (-19\%) (Supplementary Fig. 1b). Since the main regulatory step for efficient trans-activation of gene transcription involves the heterodimerization of PPAR proteins with RXR $\alpha$ in a ligand-dependent manner, the moderate reduction in PPAR protein concentrations is unlikely to mediate the drastic decrease of PPAR target gene expression in Atgl-deficient cardiac muscle or liver. Importantly, mRNA levels for PGC-1 $\alpha(-80 \%)$ and PGC-1 $\beta$ (-85\%) were also drastically decreased in cardiac muscle of AtglKO mice (Fig.1c). This decrease was specific for cardiac muscle, since mRNA levels for

Figure 2: Morphology, glycogen content, mitochondria size, and mitochondrial DNA content in cardiac muscle of WT and AtglKO mice.

a

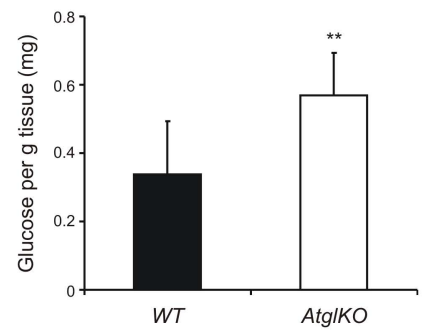

C

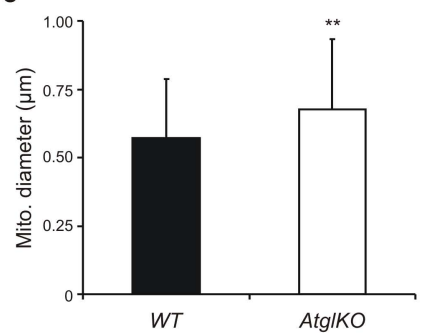

b

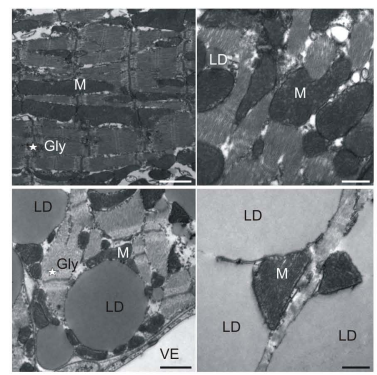

d

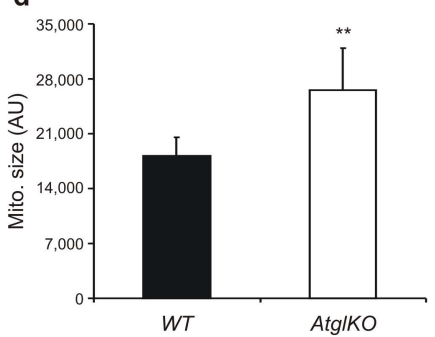

e

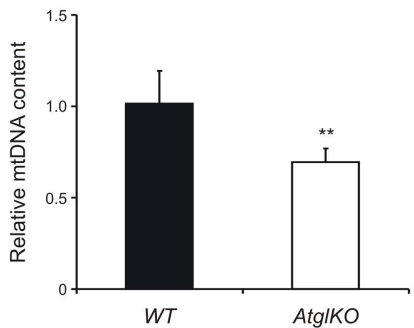

(a) Cardiac muscle glycogen content of 10-week old female WT and AtgIKO mice $(n=9)$ demonstrating increased muscle glycogen in AtgIKO mice. (b) Transmission electron microscopy of cardiac muscle sections from 10-week old female mice. WT cardiac muscle sections (upper panels) show a typical intermyofibrillar network containing mitochondria (M), glycogen (* Gly) and lipid droplets (LD). In AtgIKO cardiac muscle (lower panels) lipid droplet size and the number of glycogen granules (* Gly) embedded within the intermyofibrillar network are increased. Scale bar: $1 \mu \mathrm{m}$ for upper and lower left panel and $0.5 \mu \mathrm{m}$ upper and lower right panel. Morphometric (c) and cytofluorimetric (d) analyses of mitochondria from cardiac muscle of WT and AtgIKO mice demonstrating increased mitochondrial size in AtgIKO mice. Size was either determined from sections of 100 randomly selected mitochondria per genotype or from isolated mitochondria (FACS analysis, $n=4)$. (e) Relative mitochondrial (mt) DNA content (normalized to the single copy nuclear gene Ndufv1) in cardiac muscle of 10-week old female WT and AtgIKO mice showing reduced mitochondrial DNA content in AtgIKO mice $(n=5)$. All values are displayed as means $+\mathrm{s}$.d. $* * P<0.01$. 
PGC-1 $\alpha$ and $\beta$ were higher in the liver of AtglKO mice than in WT mice (Fig. 1d) arguing for a tissue-specific difference in the regulation of PGC-1 expression. In cardiac muscle, Atgldeficient mice exhibited normal PGC- $1 \alpha$ and PGC-1 $\beta$ mRNA levels at birth (Supplementary Fig. 1c). At weaning (14 $\pm 1 \mathrm{~d}$ after birth), however, the concentration of PGC-1 $\alpha$ mRNA decreased by $64 \%$ and PGC-1 $\beta$ tended to decrease $(-18 \%)$. This decline precedes the pronounced cardiac lipid accumulation and defective heart function.

\section{Impaired cardiac mitochondrial respiration in AtgIKO mice}

Biochemical analysis confirmed the previously observed increased cardiac TG content $\left(20-\right.$ fold $^{19}$ and revealed increased glycogen concentrations (+70\%) in cardiac muscle of AtglKO mice (Fig. 2a). Histomorphological analysis showed that these increases were due to increased size and number of lipid droplets and glycogen granules within cardiomyocytes (Fig. 2b). Furthermore, although the overall morphology of mitochondria and the structure of mitochondrial cristae appeared normal in electron microscopical analyses of AtglKO cardiac muscle, mitochondrial size was increased when higher resolution morphometric $(+18 \%)$ and cytofluorimetric (+45\%) analyses were applied (Fig. 2c, d). Concomitantly, mitochondrial DNA content in cardiac muscle was 32\% lower in AtgIKO than in WT mice indicating a decreased number of mitochondria in hearts of AtglKO mice (Fig. 2e).

Atgl deficiency prompted a severe impairment of cardiac mitochondrial function. Heart homogenates prepared from 4-week old (Fig. 3a) and 8 week-old (Fig. 3b) AtgIKO mice exhibited drastically reduced oxygen consumption under both basal and succinatestimulated conditions. Reduced oxygen consumption was irrespective of the presence of glucose (Fig. 3a, b) or palmitate (Supplementary Fig. 2a) suggesting that the substrate energy source did not affect the mitochondrial defect. $1 \mathrm{mM}$ cyanide stopped both basal and succinate-stimulated cardiac respiration excluding non-mitochondrial mechanisms of $\mathrm{O}_{2}$ consumption (data not shown). The reduction of mitochondrial oxygen consumption was similar in 4-week and 8-week old Atg/KO animals (between -63\% and -74\%) and did not correlate with the cardiac TG content (Fig. 3c), which was 4.6-fold higher in older than in younger AtgIKO mice.

Analysis of respiration in isolated cardiac mitochondria revealed a markedly decreased oxygen flux specifically to the metabolically more flexible subsarcolemmal (SS) mitochondria 22,23 (Fig. 3d, e). Both ADP driven (State 3) and uncoupled (State U) oxygen flow was decreased in AtgIKO SS mitochondria in the presence of pyruvate (-49\% and $-43 \%$, respectively) and palmitoyl-CoA (-79\% and $-78 \%$, respectively). The decrease in oxygen 
flux in the presence of both glycolytic and lipolytic substrates associated with marked derangements in the mitochondrial respiratory chain. Protein quantity for NDUFA9 (complex I; NADH dehydrogenase [ubiquinone] $1 \alpha$ subcomplex subunit 9) and SDHA (complex II; succinate dehydrogenase complex subunit A) were $75 \%$ and $29 \%$, respectively, lower in AtgIKO mitochondria than in WT mitochondria (Fig. 3f). In contrast, MTCO1 (complex IV; mitochondrial cytochrome c oxidase 1) protein quantity remained unchanged. Significantly reduced mitochondrial membrane potential (-41\%) (Fig. 3g), reduction of non-esterified free thiol groups (-51\%) (Fig. 3h), and decreased FA oxidation rates (Supplementary Fig. $2 \mathrm{~b})$ added to the mitochondrial dysfunction in AtgIKO cardiomyocytes. Thus, reduced PPAR target gene and PGC- $1 \alpha$ and $\beta$ expression in response to Atgl deficiency leads to defective OXPHOS in the hearts of AtgIKO mice. This reduced oxidative capacity may contribute to decreased substrate utilization and increased cardiac deposition of neutral lipids and glycogen in AtgIKO cardiomyocytes.

\section{PPAR activation and OXPHOS depend on cardiac-specific ATGL}

To examine whether the observed defects in PPAR signaling and substrate oxidation are specific to the absence of cardiac Atgl, we pursued two strategies: First, we analyzed conditional knockout mice lacking Atgl only in muscle by generating a mouse line with a lox $P$ flanked Atgl gene (AtgIFLOX mice) and breeding with transgenic mice expressing the Crerecombinase under the control of the muscle-specific creatine kinase promoter. Musclespecific recombination generated muscleAtgIKO mice with extremely low Atgl mRNA levels in cardiac (-95\% compared to WT mice) (Supplementary Fig. 3a) and skeletal muscle (not shown). Atgl mRNA levels in other tissues (e.g. liver) were comparable to those in WT mice. Muscle-specific Atgl deletion increased heart weight 1.5-fold (127 $\pm 5 \mathrm{mg}$ for muscleAtglKO mice versus $84 \pm 12 \mathrm{mg}$ for controls, $P<0.001, n=7$ ) and cardiac TG content 10.7-fold (Fig. 4a). mRNA expression for PPAR $\alpha$ and $\delta$ target genes in cardiac muscle of muscleAtgIKO mice decreased by 50 to $80 \%$ and PGC- $1 \alpha$ mRNA by $85 \%$ (Fig. $4 \mathrm{~b}$ ). This cardiac phenotype is indistinguishable from the one observed in conventional AtglKO mice and argues for a specific role of cardiac ATGL in PPAR regulated energy metabolism. Expectedly, and in contrast to AtgIKO mice, muscleAtgIKO mice exhibited normal hepatic mRNA levels for PPAR $\alpha$ and $\delta$ target genes (Supplementary Fig. 3b).

Second, to test whether Atgl in the heart alone is sufficient to rescue the cardiac phenotype of AtgIKO mice, we generated transgenic mice with cardiomyocyte-specific overexpression of Atgl (cmAtg/TG) (Supplementary Fig. 4a). cmAtg/TG mice were subsequently backcrossed onto an AtgIKO background yielding mice with Atgl present exclusively in cardiac muscle 
Figure 3: Oxygen consumption of cardiac muscle homogenates and isolated mitochondria, abundance of respiratory chain proteins, and mitochondrial oxidative stress parameters in cardiac muscle of WT and AtglKO mice.

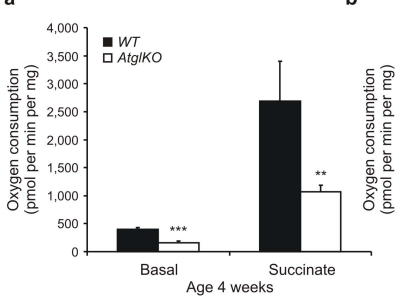

d

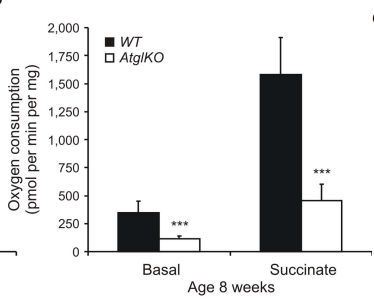

e

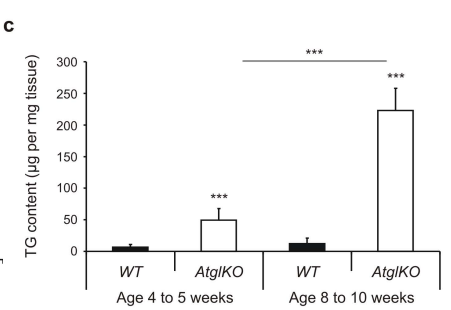

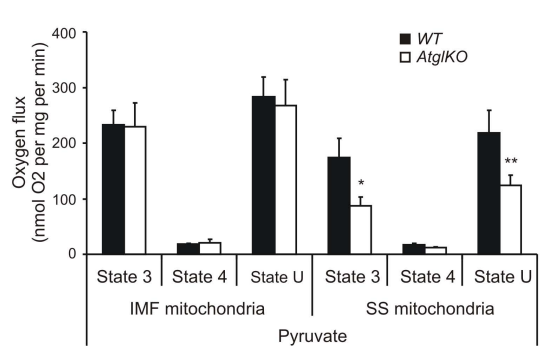
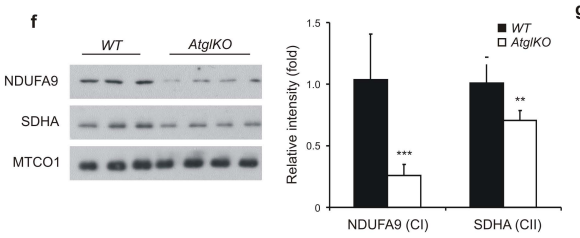

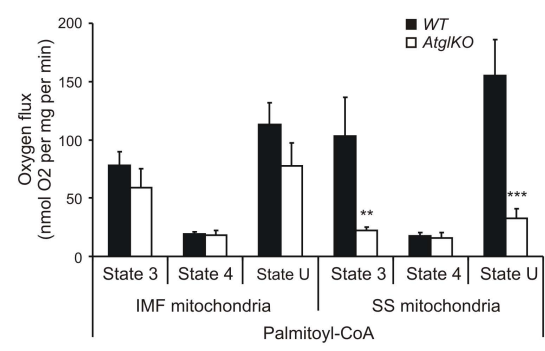

h

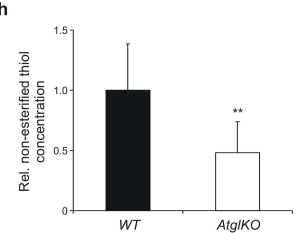

Substantially decreased oxygen consumption, an indicator for mitochondrial respiration, was observed in Atg/KO cardiac homogenates of 4-week (a) and 8-week old (b) male mice in the presence of glucose indicating impaired mitochondrial function in the absence of Atgl $(n=6)$. (c) TG content in cardiac muscle of WT and AtgIKO mice demonstrating increased cardiac steatosis with age in AtgIKO mice. (d, e) Oxygen flux of mitochondria isolated from cardiac tissue of 8 to 9-week old male WT and AtgIKO mice. ADP driven (State 3) and uncoupled (State U) oxygen flow was decreased in the presence of pyruvate (d) and palmitoyl-CoA (e) in subsarcolemmal but not in intramyfibrillar mitochondria $(n=6)$. (f) Western blotting analysis of mitochondrial respiratory chain proteins for complex I [NADH dehydrogenase (ubiquinone) $1 \alpha$ subcomplex subunit 9 (NDUFA9)], complex II [succinate dehydrogenase complex subunit A (SDHA)], and complex 4 [mitochondrial cytochrome c oxidase 1 (MTCO1)]. Cardiac mitochondria were isolated from 8 to 9-week old, female WT and AtgIKO mice and equal protein amounts were loaded onto the gel. Analysis of band intensities after their detection with specific antibodies revealed that NDUFA9 and SDHA were decreased in mitochondrial preparations of AtgIKO mice compared to WT mice. (g) Determination of mitochondrial membrane potential by tetramethyl-rhodaminemethylester perchlorate (TMRM) staining and FACS analysis of isolated mitochondria from cardiac muscle of 8 to 9-week old, female $W T$ and AtgIKO mice demonstrating reduced membrane potential in mitochondria of AtgIKO mice $(n=4)$. (h) Relative concentrations of non-oxidized ("free") thiol groups in isolated mitochondria of 8 to 9-week old female WT and Atg/KO mice. Increased oxidation of thiol groups in Atg/KO mitochondria indicated increased oxidative stress $(n=4)$. All values are displayed as means + s.d. ${ }^{*} P<0.05,{ }^{* *} P<0.01$, and ${ }^{* * *} P<0.001$. 
(designated AtgIKO-cmAtg/TG). Atgl enzyme activity in cardiac muscle of Atg/KO-cmAtg/TG mice was 5 to 7-fold higher than in control mice (Supplementary Fig. 4b). Cardiac-exclusive Atgl expression entirely rescued the lethal heart phenotype of conventional AtglKO mice and prolonged life span to periods observed in WT animals ( $>2$ years) (Supplementary Fig. 4c). Cardiac hypertrophy and massive TG accumulation, a characteristic of the Atgl-deficient heart, reverted to normal in AtgIKO-cmAtgITG animals (Fig. 4c, d) whereas adipose tissue mass remained high (Fig. 4e). Restoration of Atgl enzyme activities in cardiac muscle caused a substantial increase in PPAR $\alpha$ and $\delta$ target gene and PGC-1 $\alpha$ and $\beta$ expression (Fig. 4f), and increased oxidative capacity to values found in WT mice (Fig. 4g).

Together, these results assign a crucial and specific role to cardiac Atgl in the activation of PPAR target gene expression and lipid oxidation. To determine whether these parameters could be attributed to differences in availability of extracellular lipid substrates, we measured plasma concentrations of lipid substrates (FA and TG) and cardiac LPL enzyme activities in all mouse models and correlated these with PPAR target gene expression and cardiac phenotype. Fasted AtgIFLOX and conditional muscleAtgIKO mice exhibited normal plasma FA, TG and total cholesterol concentrations similar to those observed in fasted WT mice (Supplementary Table 1). In contrast, plasma FA and TG concentrations were much lower in fasted AtgIKO (-67\% and -66\%, respectively) and AtgIKO-cmAtgITG mice (-74\% and $-69 \%$, respectively) than in WT mice. Cardiac-specific LPL enzyme activities varied among different genotypes with highest values observed in AtgIKO and AtgIKO-cmAtg/TG mice (Supplementary Fig. 5a). In AtgIKO hearts, this led to a 53\% increase in the total intracellular concentration of unesterified FA (Supplementary Fig. 5b). Importantly, both plasma lipid concentrations and cardiac LPL activities did not correlate with PPAR target gene expression or the severity of the cardiac phenotype.

To test whether overexpression of ATGL in an in vitro cell culture system enhances PPAR $\alpha$ target gene expression, we performed reporter gene transactivation assays. We cotransfected HepG2 cells with a plasmid expressing a luciferase reporter gene under the control of multiple PPAR-responsive elements (PPREs) and an expression vector for PPAR $\alpha$. This co-transfection strongly induced luciferase reporter expression compared to PPRE/ luciferase transfected cells alone (Fig. 4h). Upon addition of exogenous linoleic acid (18:2), reporter activity increased further by $38 \%$ confirming previous reports that FA induce PPAR $\alpha$ target gene expression ${ }^{24}$. The additional overexpression of Atgl via an Atgl expression plasmid further augmented luciferase activity in the presence or absence of exogenous FA (44\% and $61 \%$, respectively). Thus, the overexpression of ATGL stimulates PPAR $\alpha$-mediated reporter gene transcription in vitro. 
Figure 4: Changes in PPAR $\alpha$ and $\delta$ activated gene expression and OXPHOS in mice lacking or overexpressing Atgl in cardiac muscle.

a

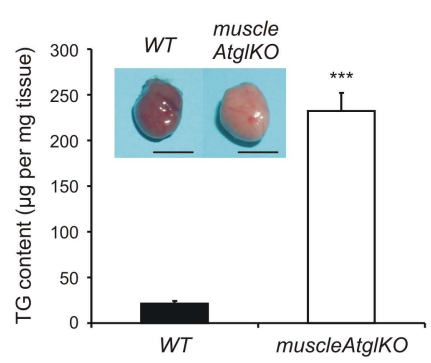

b

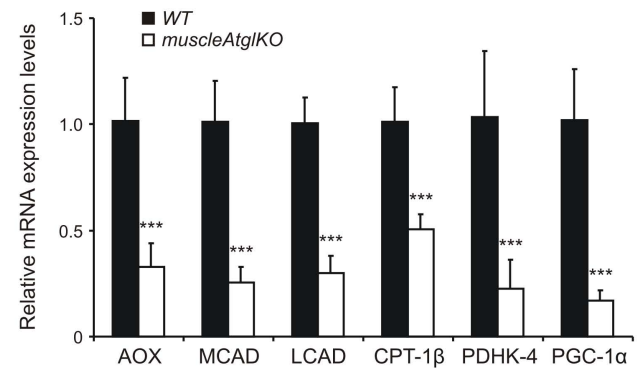

d
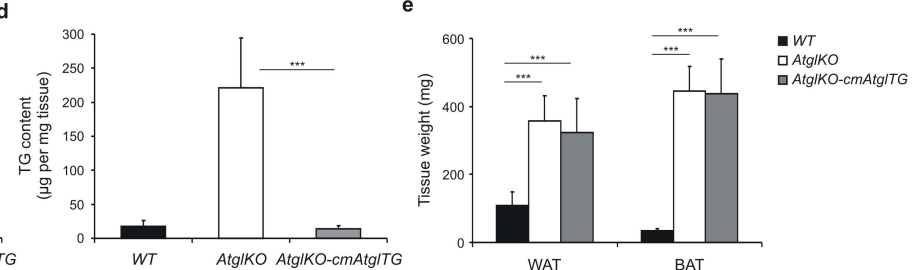

h

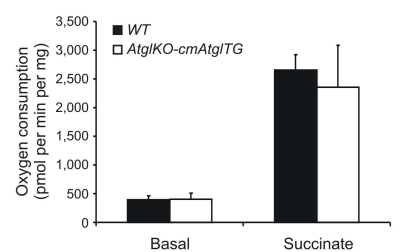

(a) Cardiac TG content in WT and conditional knockout mice lacking Atgl in cardiac and skeletal muscle (mouseAtg/KO) demonstrating a drastic cardiac steatosis in muscleAtgIKO mice $(n=5)$. Scale bar: $5 \mathrm{~mm}$. (b) mRNA expression levels of PPAR $\alpha$ and $\delta$ target genes and PGC- $1 \alpha$ are reduced in cardiac muscle of muscleAtglKO mice compared to WT mice $(n=5)$. (c) Heart weight, (d) cardiac muscle TG content, and (e) white and brown adipose tissue (WAT and BAT) weight of WT, AtgIKO, and AtgIKO-cmAtgITG mice expressing an Atg/ transgene on an AtgIKO background. Cardiacexclusive transgene expression normalizes cardiac weight and TG content to WT levels, whereas WAT and BAT weight remain as high as in AtgIKO mice $(n=6)$. (f) mRNA expression levels of PPAR $\alpha$ and $\delta$ target genes and PGC$1 \alpha$ and $\beta$ in cardiac muscle of WT, AtgIKO, and AtgIKO-cmAtgITG mice indicate reduced target gene expression in AtgIKO mice that partially recovers upon cardiac-specific $A t g /$ transgene expression $(n=4)$. (g) Oxygen consumption in cardiac homogenates prepared from 8 to 9-week old female WT and AtgIKO-cmAtgITG mice $(n=6)$. (h) Relative luciferase activities in lysates of HepG2 cells transfected with a PPRE/luciferase reporter plasmid and a PPAR $\alpha$ expression vector. The additional expression of Atgl increases luciferase activity in the absence or presence of exogenously added linoleic acid (LA). All values are displayed as means + s.d. ${ }^{*} P<0.05, * * P<0.01$ and ${ }^{* * *} P<0.001$. 


\section{PPAR $\alpha$ agonist restores cardiac respiration and function}

Next, we assessed whether the severe lipid and respiratory defects in Atgl-deficient mice can be bypassed by lipolysis-independent PPAR activation via PPAR agonists. Feeding 6 -week old female AtgIKO mice a chow diet containing $0.1 \%$ of the PPAR $\alpha$ agonist Wy14643 for three weeks decreased circulating TG and FA (Supplementary Table 2) and substantially reduced TG content in heart (-52\%) and liver (-50\%) compared to AtglKO mice fed a normal chow diet (Fig. 5a). Similarly, feeding AtglKO mice a chow diet containing $0.2 \%$ of the alternative PPAR $\alpha$ agonist fenofibrate for 10 weeks reduced the cardiac and hepatic TG contents by $62 \%$ and 49\%, respectively (Fig. 5b). Wy14643 treatment caused a substantial increase in PPAR $\alpha$ target gene expression. Notably, PGC-1 $\alpha$ and $\beta$ mRNA levels also reverted to $W T$ values (Fig. 5c). Agonist treatment improved basal and succinate-stimulated respiratory rates of AtglKO hearts to $W T$ values (Fig. 5d), normalized free thiol concentrations (Supplementary Fig. 6a), and partially restored mitochondrial size (Supplementary Fig. 6b) and membrane potential (Supplementary Fig. 6c). The beneficial effects of Wy14643 were not caused by an induction of cardiac TG hydrolase activity (Supplementary Fig. 6d).

PPAR $\alpha$ agonist treatment substantially improved heart function in AtglKO mice. In echocardiography (Fig. 5e, Supplementary Table 3), AtglKO mice exhibited a massive increase in left ventricular (LV) mass index. This was largely due to a thickening of the septal and posterior wall (without significant change in the LV end-diastolic dimension) resulting in a concentric hypertrophic phenotype. LV systolic function was significantly impaired in AtgIKO mice as indicated by an increase in LV end-systolic dimensions and a robust decrease in LV fractional shortening. A 3-week treatment with the PPAR $\alpha$ agonist Wy14643 completely reversed this phenotype. Treated AtgIKO mice had normal ventricular systolic function and a LV mass index comparable to WT animals (Fig. 5f, g). In fact, LV end-diastolic dimensions of treated AtgIKO mice were even lower than in WT animals. Thus, exogenous PPAR $\alpha$ stimulation is sufficient to markedly improve mitochondrial function and to fully restore the cardiac performance of AtglKO mice.

\section{Wy14643 prevents cardiac death and improves FA utilization}

To investigate whether PPAR $\alpha$ agonist-mediated improvement of respiratory function and cardiac performance prevents the early death of AtglKO mice, we kept 8-week old AtgIKO males on a chow diet with and without Wy14643 for 12 weeks. While all vehicle-treated knockout mice died within the first 4 weeks of the treatment period, 4 out of 5 Wy14643treated knockout animals survived the complete duration of the experiment (Fig. 6a). One animal of the Wy14643 group was bitten to death during the second week of treatment. 
Figure 5: TG content, oxygen consumption, and cardiac function in AtgIKO mice treated with PPARa agonists.
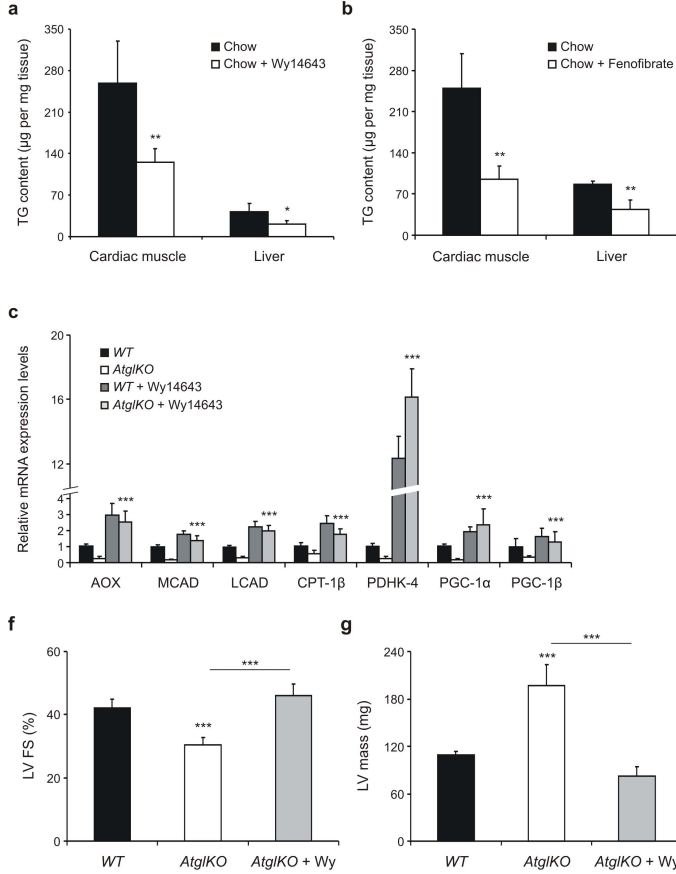

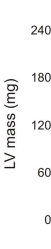

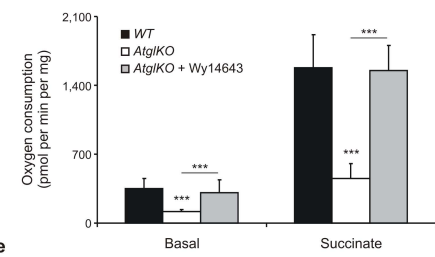

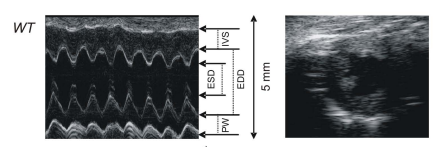

AtgIKO
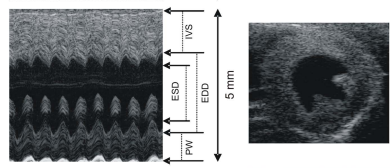

AtglKO $+\mathrm{Wy} 14643$

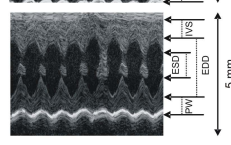

M-Mode

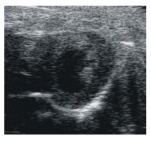

B-Mode

(a) Cardiac and hepatic TG content in 6-week old female AtgIKO mice on chow diet with or without $0.1 \%$ Wy14643 for 3 weeks. Treated AtgIKO mice showed a substantial decrease in heart and liver TG content compared to AtgIKO mice on chow alone $(n=5)$. (b) Cardiac and hepatic TG content in 6-week old female AtgIKO mice on chow diet with or without $0.2 \%$ fenofibrate for 10 weeks. Fenofibrate treatment reduced heart and liver TG in AtgIKO mice compared to Atg/KO mice on regular chow diet $(n=4-5)$. (c) mRNA expression levels of PPAR $\alpha$ and $\delta$ target genes and PGC- $1 \alpha$ and $\beta$ in cardiac muscle of female $W T$ and AtgIKO mice fed a chow diet with or without $0.1 \%$ Wy14643 for 3 weeks. Agonist treatment increased the expression of all analyzed genes in both WT and AtgIKO mice $(n=5)$. (d) Wy14643 treatment of male AtgIKO mice for 3 weeks completely restored oxygen consumption to values observed in untreated WT mice in cardiac muscle preparations under both basal conditions and succinatestimulated conditions $(n=5)$. (e) Wy14643 treatment rescued left ventricular (LV) dysfunction in female AtgIKO mice analyzed by $\mathrm{M}$ - and B-mode echocardiographic imaging. The increased septal wall (IVS) and posterior wall (PW) thickness present in AtgIKO mice returned to WT values. Similarly, increased LV end systolic dimensions (ESD) in AtgIKO mice improved to ranges seen in WT mice (M-mode imaging, left panel). Massive concentric cardiac hypertrophy present in AtgIKO mice was markedly decreased in AtgIKO mice on the agonist diet (B-mode short axis imaging, right panel). (f, g) Impaired LV fractional shortening (LV FS) and drastically increased LV mass in AtgIKO mice could be reversed to $W T$ measures. $n=5$. All values are displayed as means + s.d. ${ }^{*} P<0.05,{ }^{*} * P<0.01$ and $* * * P<0.001$. 
Remarkably, cardiac muscle and liver TG content in AtgIKO mice reverted to those seen in WT mice on this long-term treatment with the PPAR $\alpha$ agonist. TG contents also decreased in other Atgl-deficient organs including kidney, testis, and ileum (Fig. 6b).

At the same time, the physical performance of AtgIKO mice and their "metabolic flexibility" in energy substrate utilization improved after a 7-day treatment with Wy14643. During the dark cycle when the animals are more active, oxygen consumption in metabolic chambers was $12 \%$ lower in AtgIKO mice than in WT mice (Fig. 6c). This difference decreased to $9 \%$ when the animals received Wy14643. To test for "metabolic flexibility", we assessed mice fed a chow diet \pm Wy14643 by indirect calorimetry (Fig. 6d). During the dark period when animals eat, values for the respiratory quotient (RQ) were around 1.0 suggesting that glucose was the only energy substrate utilized in both genotypes. During the light period when mice typically decrease their food intake or during food deprivation $3 \mathrm{~h}$ prior to the experiment, the RQ values of WT animals dropped to 0.89 and 0.80 , respectively, indicating a switch from glucose to FA utilization. These decreases were significantly less pronounced in AtgIKO mice ( 0.92 in animals during the light cycle and 0.83 after fasting) suggesting that the animals were less efficient in switching the energy substrate from glucose to FA. Wy14643 administration significantly decreased the RQ values in both WT and AtgIKO mice. However, RQs remained higher in AtgIKO mice than in WT mice. Thus, PPAR agonist treatment increases FA utilization in both genotypes but is unable to fully restore FA oxidation in AtglKO mice to WT values. Presumably, this results from the restricted FA availability in fasted AtgIKO mice due to the lipolytic defect in white adipose tissue.

\section{PPARס agonists fail to improve the cardiometabolic defects}

In addition to PPAR $\alpha$, PPAR $\delta$ plays a prominent role in the transcriptional regulation of genes involved in FA uptake and oxidation in many tissues including cardiac and skeletal muscle ${ }^{25-27}$. To differentiate between PPAR $\alpha$ and PPAR $\delta$-mediated effects, we investigated whether the administration of the well-established PPAR $\delta$ agonists GW501516 ${ }^{27}$ and GW0742 ${ }^{28}$ can also improve the cardiometabolic defects in Atg/KO mice. Consistent with previous observations ${ }^{27}$, daily intraperitoneal administration of GW501516 at $5 \mu \mathrm{g}$ per gram body weight to 7-week old mice for 2 weeks decreased hepatic TG content in WT and AtglKO mice $(-37 \% \%$ and $-29 \%, p<0.08$, respectively) (Supplementary Fig. 7a) and increased the expression of PPAR $\alpha$ and $\delta$ target genes to $W T$ values (Supplementary Fig. $7 \mathrm{~b}$ ). Unexpectedly, however, PPAR $\delta$ agonist treatment did not improve the cardiac phenotype of AtglKO mice. GW501516 increased rather than decreased the cardiac TG content (Supplementary Fig. 7c) in both WT (2.2-fold) and AtgIKO mice (1.3-fold). PPAR target gene expression (Supplementary Fig. 7d) 
and cardiac oxygen consumption (Supplementary Fig. 7e) remained unaffected. Application of GW501516 by oral gavage ( $5 \mu \mathrm{g} / \mathrm{g}$ body weight) twice a day for 4 days yielded similar results. Whereas hepatic mRNA levels for PPAR $\delta$ targets in AtglKO mice increased to normal (Supplementary Fig. 7f), cardiac PPARס target gene expression (Supplementary Fig. 7g) and TG content (Supplementary Fig. 7h) remained abnormal. Cardiac PGC-1 $\alpha$ and PGC-1 $\beta$ mRNA concentrations slightly increased in WT and AtgIKO mice in response to GW501516, however, in treated AtgIKO mice they remained 60\% below those observed in untreated WT mice (Supplementary Fig. 7i). In contrast, hepatic PGC-1 $\alpha$ and $\beta$ mRNA levels were

Figure 6: Life span, tissue TG content, and energy substrate utilization in WT and AtgIKO mice treated with the PPARa agonist Wy14643.

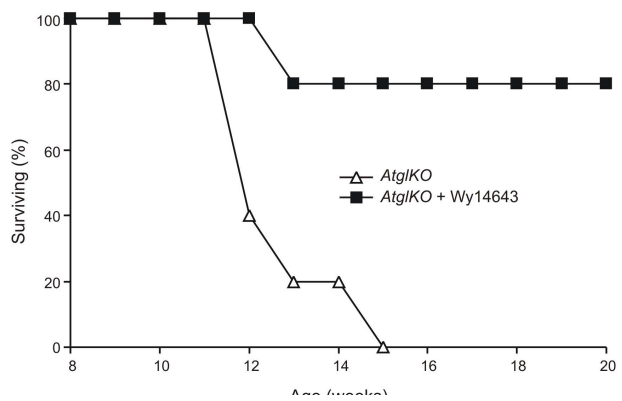

b

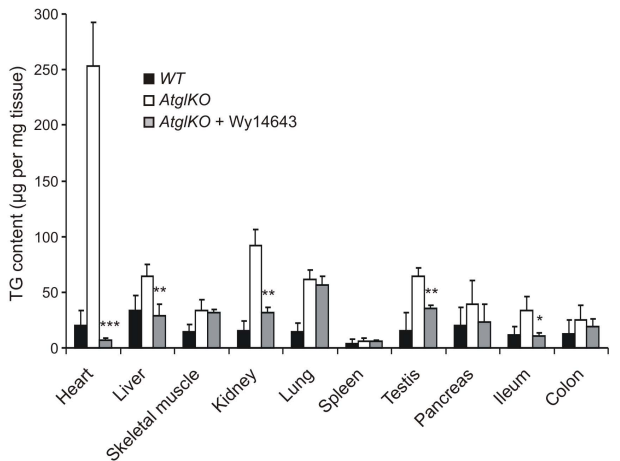

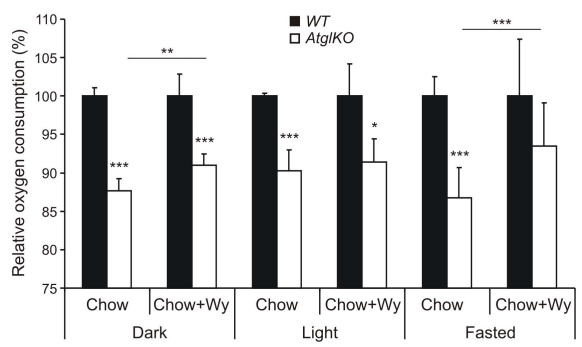

d

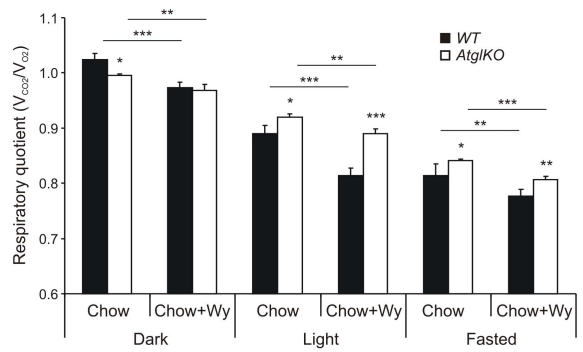

(a) Treatment of 8-week old AtgIKO mice on chow diet containing 0.1\% WY14643 for 12 weeks prevented cardiac death and (b) lowered tissue TG content including those in cardiac muscle and liver to those observed in WT animals $(n=4)$. (c) Relative whole body oxygen consumption of 8 to 9-week old, female WT and AtglKO mice housed in metabolic cages $(n=5)$. AtgIKO mice consumed less oxygen under all conditions. Wy14643 administration for 1 week increased oxygen consumption in AtglKO mice during the dark cycle and during fasting. (d) Respiratory quotients ( $R Q$, calculated from the ratio of carbon dioxide elimination versus oxygen consumption) were increased in AtgIKO mice compared to WT during the light period and in the fasted state indicating preferential glucose utilization as oxidative fuel. Upon Wy14643 treatment, RQ values significantly decreased in both WT and AtgIKO mice, although values were still increased in AtglKO mice compared to controls $(n=5)$. All values are displayed as means + s.d. $* P<0.05, * * P<0.01$ and $* * * P<0.001$. 
consistently higher in AtgIKO than in WT mice independently of PPAR $\delta$ agonist treatment (Fig. 1d and Supplementary Fig. 7j). Oral administration of the alternative PPAR $\delta$ agonist GW0742 also had no effect on cardiac PPAR target gene expression, tissue TG content, plasma lipid parameters, or body weight (not shown). Thus, treatment with the PPAR $\delta$ agonists GW501516 and GW0742 failed to rescue the metabolic and oxidative defect in cardiac muscle of AtgIKO mice.

Figure 7: Scheme of the integration of ATGL-mediated lipolysis in PPAR signaling.

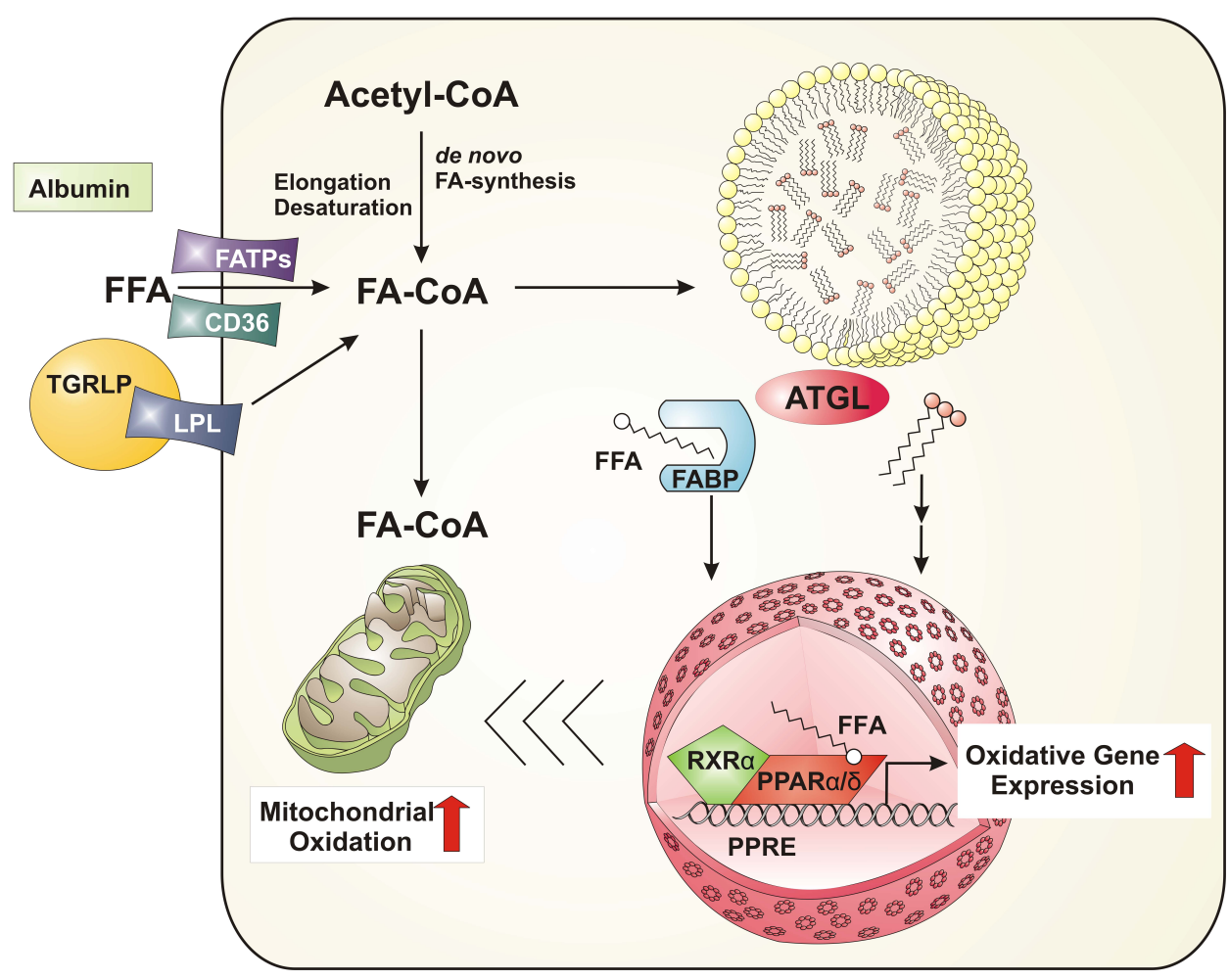

FA from exogenous or endogenous sources are not available as ligands for nuclear receptor signaling but instead are activated to acyl-CoAs and subsequently oxidized or esterified to TG. ATGL-mediated lipolysis of TG stores preferentially generates ligands or precursors of ligands for nuclear receptors controlling mitochondrial function and OXPHOS. Abbreviations: ATGL, adipose triglyceride lipase; CD36, cluster of differentiation-36; CoA, coenzyme A; FABP, FA binding protein; FATP, fatty acid transport protein; FFA, free fatty acid; LPL, lipoprotein lipase; PPAR, peroxisome proliferator-activated receptor; PPRE, PPAR response element; RXR, retinoic $X$ receptor; TGRLP, triglyceride-rich lipoproteins. 


\section{Discussion}

This study shows that the activation of cardiac and hepatic gene transcription by the PPAR $\alpha$ PGC-1 complex depends on the lipolytic catabolism of cellular TG depots. This process requires the hydrolytic activity of Atgl. The absence of this enzyme in cardiac muscle of conventional AtgIKO mice causes a lipolytic defect resulting in massive lipid accumulation, drastic reduction of PPAR $\alpha$-regulated gene expression, defective mitochondrial substrate oxidation, severe cardiac dysfunction, and premature death. A similar phenotype is observed in conditional knockout mice lacking Atgl only in cardiac and skeletal muscle (muscleAtglKO) indicating that the defects are specific to the absence of Atgl in cardiomyocytes. Concurrent analysis of the liver of AtgIKO mice confirmed previous observations demonstrating that AtgI regulates TG turnover and the expression of PPAR $\alpha$ target genes ${ }^{29-31}$. In humans, ATGLdeficiency provokes a similar clinical phenotype: affected individuals develop NLSD with severe cardiomyopathy that requires cardiac transplantation in a subgroup of patients. The pronounced phenotype in energy homeostasis and cardiac function is specific for Atgl deficiency because (i) $\mathrm{Hsl}$ or other potential lipid hydrolases cannot compensate for the absence of this enzyme and (ii) all metabolic and functional derangements in the heart can be corrected by the cardiac-specific expression of an Atg/ transgene in mice that lack Atgl in all other tissues. Thus, the Atgl-mediated generation of lipolytic products is an indispensable requirement for the regulation of PPAR $\alpha$ target genes and represents a novel mechanism for gene regulation. It supports the concept that lipid droplets are not only a cellular compartment for energy storage but also serve as a reservoir for specific lipid mediators, which require lipolysis for their spatiotemporally coordinated release and subsequent action.

FA as potential ligands for PPAR $\alpha$ activity originate from two major sources. Endogenously synthesized FA ("new fat") ${ }^{14}$ predominantly regulate hepatic PPAR $\alpha$ activity. In contrast, exogenous FA derived from adipose tissue (FA-albumin complexes) or FA derived from the hydrolysis of hepatic VLDL particles via LPL ("old fat") ${ }^{1}$ regulate cardiac PPAR $\alpha$ activity. Changes in liver-specific FA synthase or cardiac-specific LPL activities in mutant mouse models strongly affect energy substrate usage, PPAR signaling, and cellular lipid homeostasis ${ }^{14,32-34}$ proving the crucial role of these enzymes in the delivery of PPAR ligands. Our data add an additional aspect to these findings: Functional PPAR signaling requires Atglcatalyzed hydrolysis of intracellular TG stores generated from endogenous or exogenous FA. Apparently, FA must first be esterified to TG and re-hydrolyzed by ATGL before they become active signaling lipids. Ligand delivery via LPL and FA uptake is indispensable but by itself not sufficient for normal PPAR signaling and cardio-metabolic function. Consistent with this 
conclusion, conventional AtgIKO mice (with decreased plasma FA and increased cardiac LPL activity) and conditional muscleAtgIKO mice (with normal plasma FA and decreased cardiac LPL activity) exhibited impaired PPAR target gene expression and lethal cardiomyopathy, whereas AtgIKO-cmAtg/TG mice (with decreased plasma FA and increased cardiac LPL activity) showed improved target gene expression and no apparent cardiomyopathy. Whether enhanced ligand delivery to the heart by the tissue-specific overexpression of $L P L^{33}$ or the fatty acid transporter $\mathrm{CD}^{36} 6^{36}$ can overcome the cardio-metabolic phenotype of Atgl deficiency remains to be assessed.

Figure 7 summarizes our current concept: Exogenous FA from FA-albumin complexes or TG-rich lipoproteins enter the cell and are coenzyme-A activated by cell membrane-bound acyl-CoA synthetases and/or FA transport proteins. Depending on energy demand, these FACoAs either enter mitochondria for oxidation or are reesterified and stored in lipid droplet TG. The generation of lipolytic products such as FA or diacylglycerols (DG) by ATGL provides ligands or ligand precursors for functional signaling by the PPAR $\alpha$-PGC-1 complex, which, in turn, activates mitochondrial biogenesis and OXPHOS and directs FA-CoAs to oxidation. Conversely, downregulation or absence of ATGL leads to decreased mitochondrial biogenesis and function and directs FA-CoAs towards TG synthesis and accumulation.

The current study did not address the molecular nature of mediators that regulate the PPAR $\alpha$-PGC-1 complex after lipolysis. Accordingly, any considerations of why lipolysis is required for the production of these mediators remain speculative. The molecular identity of PPAR ligands and their transduction from the cytoplasma to the nucleus is still a matter of intense debate. A widely accepted model involves the binding of mono- and polyunsaturated FA to cytoplasmic FA-binding protein (FABPs), translocation into the nucleus, and binding to PPAR-RXR heterodimers thereby accomplishing transcriptional activation ${ }^{24,35}$. Alternatively, it has been proposed that FA- or DG-derivatives such as prostaglandins $\mathrm{J}^{9,11}$ or 1-palmitoyl2-oleyl-sn-glycerol-3-phosphocholine ${ }^{12}$, respectively, act as PPAR ligands. ATGL-mediated hydrolysis of TG is compatible with both mechanisms because it generates unesterified FA and DG as potential lipid ligands or ligand precursors. Finally, it is even conceivable that components of lipid droplets such as lipid droplet-associated proteins dissociate during lipolysis and regulate PPAR signaling.

Atgl deficiency results in decreased expression of PGC- $1 \alpha$ and PGC-1 $\beta$ in cardiac muscle. Consistent with the established role of PGC-1 $\alpha / \beta$ in cardiac mitochondrial biogenesis, substrate oxidation, and prevention of oxidative stress ${ }^{36,37}$, low cardiac PGC-1 concentrations 
may contribute to decreased numbers of mitochondria, decreased amounts of respiratory complex I and II proteins, accumulation of reactive oxygen species, and impaired OXPHOS in cardiac muscle of AtglKO mice. Decreased mitochondrial oxygen consumption was specific to SS mitochondria, which are highly sensitive to cellular PGC-1 $\alpha$ concentrations $22,23,38$. The molecular mechanism whereby defective lipolysis suppresses cardiac PGC-1 $\alpha$ and $\beta$ expression is currently unknown. Multiple physiological stimuli including FA regulate PGC-1 $\alpha$ expression and activity via numerous signaling pathways and post-translational modifications ${ }^{7,39-41}$. A plausible mechanism suggests a direct regulation of PGC- $1 \alpha$ by lipolysisdependent PPAR $\alpha$ activation. Hondares et al. ${ }^{42}$ characterized a functional PPRE within the PGC-1 $\alpha$ promoter that is regulated by PPAR $\alpha$ agonists and Duncan et al. ${ }^{43}$ demonstrated that the upregulation of mitochondrial biogenesis and function in a mouse model with insulin resistance depends on the PPAR $\alpha$-mediated upregulation of PGC-1 $\alpha$. Our data are consistent with these observations and suggest that lipolysis regulates PGC- $1 \alpha$ via PPAR $\alpha$ signaling. Not unexpectedly, therefore, the cardiac phenotye in AtglKO was more severe than in mouse lines with individual deficiencies for PGC-1 $\alpha$ or PGC-1 $\beta$, but less severe than in a mouse models with combined PGC-1 $\alpha \beta$-deficiency ${ }^{7,44}$. The latter animals die within $24 \mathrm{~h}$ after birth from cardiac mitochondrial dysfunction and heart failure ${ }^{44}$. Whether alternative mechanisms involving additional nuclear receptors (e.g. the estrogen-related receptor, ERR or the retinoid-related orphan receptors, ROR) or non-nuclear receptors such as the nuclear respiratory factors (NRFs) also contribute to decreased cardiac PGC-1 expression requires clarification. Interestingly, PGC- $1 \alpha$ and $\beta$ expression in the liver of AtglKO mice was higher than in WT mice despite decreased PPAR $\alpha$ and $\delta$ target gene expression. This unexpected finding suggests basic tissue-specific differences in the regulation of PGC-1 expression in the heart and the liver that remain to be elucidated.

Importantly, treatment of AtgIKO mice with PPAR $\alpha$ agonists bypassed the lipolytic defect and prevented the adverse metabolic alterations and oxidative dysfunction in the heart. Wy14643 treatment of mice was previously shown to stimulate PPAR $\alpha$-activated gene expression and mitochondrial oxidation thereby reducing cellular TG content in cardiac muscle and liver ${ }^{45-47}$. In AtgIKO mice, Wy14643 treatment restored the cardiac expression of PPAR $\alpha$ target genes and PGC- $1 \alpha$ and $\beta$, reversed excessive systemic lipid accumulation, normalized oxidative function and oxidative stress in mitochondria, improved cardiac performance, and prevented cardiac death. The normalization of cardiac steatosis in response to the PPAR $\alpha$ agonist occurred without increasing cellular TG hydrolysis arguing against an induction of alternative lipase(s). The reduction in TG content may be mostly secondary to increased oxidation of exogenous FA and altered kinetics of the intramyocellular 
TG pool. In contrast, PPAR $\delta$ agonist treatment of AtgIKO mice via intraperitoneal injection or oral gavage did neither reduce cardiac lipid content or normalize PGC- $1 \alpha / \beta$ and PPAR target gene expression. This was unexpected since PPAR $\delta$ activation is associated with increased FA oxidation in muscle and liver ${ }^{27,48}$. PPAR $\delta$ agonist effects on cardiac TG content have not been reported, but mice lacking PPAR $\delta$ suffer from mild cardiac steatosis (normal cardiac TG in 2-month old animals and a 2-fold increase when they are 9 months old) and decreased OXPHOS in cardiac muscle ${ }^{25}$. Our results indicate that defective PPAR $\alpha$ activation and decreased PGC- $1 \alpha$ and $\beta$ expression account for most of the cardiometabolic derangements observed in Atgl-deficient mice and that PPAR $\delta$ activation alone is not sufficient to overcome these defects in AtgIKO mice. In the liver, administration of PPAR $\delta$ agonists normalized PPAR target gene expression. The agonist-mediated induction of target mRNAs was independent of PGC- $1 \alpha$ and $\beta$ expression, which was consistently higher in the liver of AtglKO than of WT mice. These findings argue for remarkable tissue-specific differences in cardiac muscle and liver concerning the regulation of the PPAR-PGC-1 complex by lipolysis.

The possibility that the pharmacological activation of PPAR $\alpha$ can bypass the ATGL pathway offers a potential treatment for patients with NLSD. Two distinct genetic defects are known to cause NLSD ${ }^{49}$ : Mutations in ATGL cause NLSD with myopathy (NLSDM), whereas mutations in the ATGL activator CGI-58 cause NLSD with ichthyosis (NLSDI). Both conditions cause ectopic systemic lipid storage although not to the same extent ${ }^{50}$. NLSDM patients accumulate large amounts of TG particularly in cardiac and skeletal muscle and develop only mild hepatosteatosis. Patients with NLSDI suffer from more severe hepatosteatosis and accrue less TG in muscle. If our results in AtgIKO mice also apply to human patients, treatment with PPAR $\alpha$ agonists will reduce ectopic lipid accumulation and improve organ dysfunction in these individuals. Fenofibrate, an approved and well-established activator of PPAR $\alpha^{51}$ may be particularly beneficial for the treatment of liver steatosis in NLSDI. Currently, there is no clinical evidence that PPAR $\alpha$ agonists also affect cardiac lipid metabolism and heart function in humans (unlike in the mouse ${ }^{52}$ ). Instead, human skeletal muscle and cardiac energy metabolism may rely more on the activity of PPAR $\delta^{3,53}$. Accordingly, administration of recently available PPAR $\delta$ or non-selective PPAR agonists may be an effective measure to combat excessive skeletal and cardiac lipid deposition and heart failure in patients with NLSDM. 


\section{Acknowledgements}

This research was supported by the following grants: "GOLD - Genomics of Lipid-Associated Disorders" as part of the Austrian Genome Project "GEN-AU" funded by FFG and BMWF; "SFB LIPOTOX" F30, "Doktoratskolleg: Molecular Enzymology" W901, the "Wittgenstein Award 2007" Z136, and research grant P20602 funded by the Austrian Science Fund (FWF); "TOBI" contract nr. 201608 and "LipidomicNet" contract nr. 202272 funded by the European Commission; additional funding for the SFB LIPOTOX was granted by the County of Styria and the City of Graz. P.S. is supported by a OVICl${ }^{1}$ Research Grant for innovative research from the Netherlands Organization for Scientific Research (Grant 918.96.618). T.v.d. W. was supported by the Center for Translational Molecular Medicine (CTMM) project PREDICCt (Grant 01C104) and the Netherlands Heart Foundation, Dutch Diabetes Research Foundation, and Dutch Kidney Foundation. We thank Dr. E. Zechner and C. Schober-Trummler for proofreading the manuscript and Dr. S. Lang for the preparation of the cartoon. 


\section{References}

1. Madrazo, J.A. \& Kelly, D.P. The PPAR trio: regulators of myocardial energy metabolism in health and disease. J Mol Cell Cardiol 44, 968-975 (2008).

2. Lefebvre, P., Chinetti, G., Fruchart, J.C. \& Staels, B. Sorting out the roles of PPAR alpha in energy metabolism and vascular homeostasis. J Clin Invest 116, 571-580 (2006).

3. Barish, G.D., Narkar, V.A. \& Evans, R.M. PPAR delta: a dagger in the heart of the metabolic syndrome. J Clin Invest 116, 590-597 (2006).

4. Tontonoz, P. \& Spiegelman, B.M. Fat and beyond: the diverse biology of PPARgamma. Annu Rev Biochem 77, 289-312 (2008).

5. Lehman, J.J., et al. Peroxisome proliferator-activated receptor gamma coactivator-1 promotes cardiac mitochondrial biogenesis. J Clin Invest 106, 847-856 (2000).

6. Puigserver, P., et al. A cold-inducible coactivator of nuclear receptors linked to adaptive thermogenesis. Cell 92, 829-839 (1998).

7. Rowe, G.C., Jiang, A. \& Arany, Z. PGC-1 coactivators in cardiac development and disease. Circ Res 107, 825-838.

8. Forman, B.M., Chen, J. \& Evans, R.M. Hypolipidemic drugs, polyunsaturated fatty acids, and eicosanoids are ligands for peroxisome proliferator-activated receptors alpha and delta. Proc Natl Acad Sci U S A 94, 4312-4317 (1997).

9. Kliewer, S.A., et al. Fatty acids and eicosanoids regulate gene expression through direct interactions with peroxisome proliferator-activated receptors alpha and gamma. Proc Natl Acad Sci U S A 94, 4318-4323 (1997).

10. Krey, G., et al. Fatty acids, eicosanoids, and hypolipidemic agents identified as ligands of peroxisome proliferator-activated receptors by coactivator-dependent receptor ligand assay. Mol Endocrinol 11, 779-791 (1997).

11. Yu, K., et al. Differential activation of peroxisome proliferator-activated receptors by eicosanoids. J Biol Chem 270, 23975-23983 (1995).

12. Chakravarthy, M.V., et al. Identification of a physiologically relevant endogenous ligand for PPARalpha in liver. Cell 138, 476-488 (2009).

13. Ziouzenkova, O., et al. Lipolysis of triglyceride-rich lipoproteins generates PPAR ligands: evidence for an antiinflammatory role for lipoprotein lipase. Proc Natl Acad Sci U S A 100, 2730-2735 (2003).

14. Chakravarthy, M.V., et al. "New" hepatic fat activates PPARalpha to maintain glucose, lipid, and cholesterol homeostasis. Cell Metab 1, 309-322 (2005).

15. Zechner, R., Kienesberger, P.C., Haemmerle, G., Zimmermann, R. \& Lass, A. Adipose triglyceride lipase and the lipolytic catabolism of cellular fat stores. J Lipid Res 50, 3-21 (2009).

16. Osuga, J., et al. Targeted disruption of hormone-sensitive lipase results in male sterility and adipocyte hypertrophy, but not in obesity. Proc Natl Acad Sci U S A 97, 787-792 (2000).

17. Haemmerle, G., et al. Hormone-sensitive lipase deficiency in mice causes diglyceride accumulation in adipose tissue, muscle, and testis. J Biol Chem 277, 4806-4815 (2002).

18. Pinent, M., et al. Differential transcriptional modulation of biological processes in adipocyte triglyceride lipase and hormone-sensitive lipase-deficient mice. Genomics 92, 26-32 (2008).

19. Haemmerle, G., et al. Defective lipolysis and altered energy metabolism in mice lacking adipose triglyceride lipase. Science 312, 734-737 (2006). 
20. Fischer, J., et al. The gene encoding adipose triglyceride lipase (PNPLA2) is mutated in neutral lipid storage disease with myopathy. Nat Genet 39, 28-30 (2007).

21. Hirano, K., Ikeda, Y., Zaima, N., Sakata, Y. \& Matsumiya, G. Triglyceride deposit cardiomyovasculopathy. N Engl J Med 359, 2396-2398 (2008).

22. Benton, C.R., et al. Modest PGC-1alpha overexpression in muscle in vivo is sufficient to increase insulin sensitivity and palmitate oxidation in subsarcolemmal, not intermyofibrillar, mitochondria. J Biol Chem 283, 4228-4240 (2008).

23. Holloway, G.P., Gurd, B.J., Snook, L.A., Lally, J. \& Bonen, A. Compensatory increases in nuclear PGC1alpha protein are primarily associated with subsarcolemmal mitochondrial adaptations in ZDF rats. Diabetes 59, 819-828.

24. Wolfrum, C., Borrmann, C.M., Borchers, T. \& Spener, F. Fatty acids and hypolipidemic drugs regulate peroxisome proliferator-activated receptors alpha - and gamma-mediated gene expression via liver fatty acid binding protein: a signaling path to the nucleus. Proc Natl Acad Sci U S A 98, 2323-2328 (2001).

25. Cheng, L., et al. Cardiomyocyte-restricted peroxisome proliferator-activated receptor-delta deletion perturbs myocardial fatty acid oxidation and leads to cardiomyopathy. Nat Med 10, 1245-1250 (2004).

26. Lee, C.H., et al. PPARdelta regulates glucose metabolism and insulin sensitivity. Proc Natl Acad Sci U S A 103, 3444-3449 (2006).

27. Tanaka, T., et al. Activation of peroxisome proliferator-activated receptor delta induces fatty acid beta-oxidation in skeletal muscle and attenuates metabolic syndrome. Proc Natl Acad Sci U S A 100, 15924-15929 (2003).

28. Sznaidman, M.L., et al. Novel selective small molecule agonists for peroxisome proliferatoractivated receptor delta (PPARdelta)--synthesis and biological activity. Bioorg Med Chem Lett 13, 1517-1521 (2003).

29. Reid, B.N., et al. Hepatic overexpression of hormone-sensitive lipase and adipose triglyceride lipase promotes fatty acid oxidation, stimulates direct release of free fatty acids, and ameliorates steatosis. J Biol Chem 283, 13087-13099 (2008).

30. Ong, K.T., Mashek, M.T., Bu, S.Y., Greenberg, A.S. \& Mashek, D.G. Adipose triglyceride lipase is a major hepatic lipase that regulates triacylglycerol turnover and fatty acid signaling and partitioning. Hepatology.

31. Sapiro, J.M., Mashek, M.T., Greenberg, A.S. \& Mashek, D.G. Hepatic triacylglycerol hydrolysis regulates peroxisome proliferator-activated receptor alpha activity. J Lipid Res 50, 1621-1629 (2009).

32. Augustus, A., et al. Cardiac-specific knock-out of lipoprotein lipase alters plasma lipoprotein triglyceride metabolism and cardiac gene expression. J Biol Chem 279, 25050-25057 (2004).

33. Yagyu, H., et al. Lipoprotein lipase (LpL) on the surface of cardiomyocytes increases lipid uptake and produces a cardiomyopathy. J Clin Invest 111, 419-426 (2003).

34. Duncan, J.G., et al. Rescue of cardiomyopathy in peroxisome proliferator-activated receptoralpha transgenic mice by deletion of lipoprotein lipase identifies sources of cardiac lipids and peroxisome proliferator-activated receptor-alpha activators. Circulation 121, 426-435.

35. Tan, N.S., et al. Selective cooperation between fatty acid binding proteins and peroxisome proliferator-activated receptors in regulating transcription. Mol Cell Biol 22, 5114-5127 (2002).

36. St-Pierre, J., et al. Suppression of reactive oxygen species and neurodegeneration by the PGC-1 transcriptional coactivators. Cell 127, 397-408 (2006). 
37. Valle, I., Alvarez-Barrientos, A., Arza, E., Lamas, S. \& Monsalve, M. PGC-1alpha regulates the mitochondrial antioxidant defense system in vascular endothelial cells. Cardiovasc Res 66, 562-573 (2005).

38. Hood, D.A. Invited Review: contractile activity-induced mitochondrial biogenesis in skeletal muscle. J Appl Physiol 90, 1137-1157 (2001).

39. Sugden, M.C., Caton, P.W. \& Holness, M.J. PPAR control: it's SIRTainly as easy as PGC. J Endocrinol 204, 93-104.

40. Lin, J., Handschin, C. \& Spiegelman, B.M. Metabolic control through the PGC-1 family of transcription coactivators. Cell Metab 1, 361-370 (2005).

41. Hock, M.B. \& Kralli, A. Transcriptional control of mitochondrial biogenesis and function. Annu Rev Physiol 71, 177-203 (2009).

42. Hondares, E., et al. Thiazolidinediones and rexinoids induce peroxisome proliferator-activated receptor-coactivator (PGC)-1alpha gene transcription: an autoregulatory loop controls PGC1alpha expression in adipocytes via peroxisome proliferator-activated receptor-gamma coactivation. Endocrinology 147, 2829-2838 (2006).

43. Duncan, J.G., Fong, J.L., Medeiros, D.M., Finck, B.N. \& Kelly, D.P. Insulin-resistant heart exhibits a mitochondrial biogenic response driven by the peroxisome proliferator-activated receptor-alpha/PGC-1alpha gene regulatory pathway. Circulation 115, 909-917 (2007).

44. Lai, L., et al. Transcriptional coactivators PGC-1alpha and PGC-lbeta control overlapping programs required for perinatal maturation of the heart. Genes Dev 22, 1948-1961 (2008).

45. Aoyama, T., et al. Altered constitutive expression of fatty acid-metabolizing enzymes in mice lacking the peroxisome proliferator-activated receptor alpha (PPARalpha). J Biol Chem 273, 5678-5684 (1998).

46. Chou, C.J., et al. WY14,643, a peroxisome proliferator-activated receptor alpha (PPARalpha) agonist, improves hepatic and muscle steatosis and reverses insulin resistance in lipoatrophic A-ZIP/F-1 mice. J Biol Chem 277, 24484-24489 (2002).

47. Ye, J.M., et al. Peroxisome proliferator-activated receptor (PPAR)-alpha activation lowers muscle lipids and improves insulin sensitivity in high fat-fed rats: comparison with PPARgamma activation. Diabetes 50, 411-417 (2001).

48. Cheng, L., et al. Peroxisome proliferator-activated receptor delta activates fatty acid oxidation in cultured neonatal and adult cardiomyocytes. Biochem Biophys Res Commun 313, 277-286 (2004).

49. Schweiger, M., Lass, A., Zimmermann, R., Eichmann, T.O. \& Zechner, R. Neutral lipid storage disease: genetic disorders caused by mutations in adipose triglyceride lipase/PNPLA2 or CGI58/ABHD5. Am J Physiol Endocrinol Metab 297, E289-296 (2009).

50. Igal, R.A., Rhoads, J.M. \& Coleman, R.A. Neutral lipid storage disease with fatty liver and cholestasis. J Pediatr Gastroenterol Nutr 25, 541-547 (1997).

51. Rosenson, R.S. Fenofibrate: treatment of hyperlipidemia and beyond. Expert Rev Cardiovasc Ther 6, 1319-1330 (2008).

52. Vikramadithyan, R.K., et al. Peroxisome proliferator-activated receptor agonists modulate heart function in transgenic mice with lipotoxic cardiomyopathy. J Pharmacol Exp Ther 313, 586-593 (2005).

53. Wang, Y.X., et al. Peroxisome-proliferator-activated receptor delta activates fat metabolism to prevent obesity. Cell 113, 159-170 (2003). 
54. Bruning, J.C., et al. A muscle-specific insulin receptor knockout exhibits features of the metabolic syndrome of NIDDM without altering glucose tolerance. Mol Cell 2, 559-569 (1998).

55. Gulick, J., Subramaniam, A., Neumann, J. \& Robbins, J. Isolation and characterization of the mouse cardiac myosin heavy chain genes. J Biol Chem 266, 9180-9185 (1991).

56. Zhao, G., Zhang, X., Xu, X., Wolin, M.S. \& Hintze, T.H. Depressed modulation of oxygen consumption by endogenous nitric oxide in cardiac muscle from diabetic dogs. Am J Physiol Heart Circ Physiol 279, H520-527 (2000).

57. Blattner, J.R., He, L. \& Lemasters, J.J. Screening assays for the mitochondrial permeability transition using a fluorescence multiwell plate reader. Anal Biochem 295, 220-226 (2001).

58. Eisenberg, T., et al. Induction of autophagy by spermidine promotes longevity. Nat Cell Biol 11, 1305-1314 (2009).

59. Nemoto, Y., et al. Altered expression of fatty acid-metabolizing enzymes in aromatasedeficient mice. J Clin Invest 105, 1819-1825 (2000).

60. Song, Q., et al. Rescue of cardiomyocyte dysfunction by phospholamban ablation does not prevent ventricular failure in genetic hypertrophy. J Clin Invest 111, 859-867 (2003). 



\section{Abstract}

Adipose triglyceride lipase (ATGL) is a lipolytic enzyme, highly specific for triglyceride hydrolysis. The ATGL knockout mouse (ATGL ${ }^{-1}$ ) accumulates lipid droplets in various tissues, including skeletal muscle and has poor maximal running velocity and endurance capacity. In this study, we tested whether abnormal lipid accumulation in skeletal muscle impairs mitochondrial oxidative phosphorylation, and hence explains the poor muscle performance of $\mathrm{ATGL}^{-1}$ mice. In vivo ${ }^{1} \mathrm{H}$ magnetic resonance spectroscopy of the tibialis anterior of $\mathrm{ATGL}^{-}$ 1- mice revealed that its intramyocellular lipid pool is $\sim 6$ fold higher than in WT controls $(p=0.0007)$. In skeletal muscle of $\mathrm{ATGL}^{-\%}$ mice glycogen content was decreased by $30 \%$ $(\mathrm{p}<0.05)$. In vivo ${ }^{31} \mathrm{P}$ magnetic resonance spectra of resting muscles showed that WT and $\mathrm{ATGL}^{-}$ I- mice have a similar energy status: [PCr], [Pi], PCr/ATP ratio, $\mathrm{PCr} / \mathrm{Pi}$ ratio, and intracellular $\mathrm{pH}$. Electro-stimulated muscles from WT and $\mathrm{ATGL}^{-1-}$ mice showed the same $\mathrm{PCr}$ depletion and $\mathrm{pH}$ reduction. Moreover, the mono-exponential fitting of the $\mathrm{PCr}$ recovery curve yielded similar PCr recovery times, $\mathrm{TPCr}\left(54.1 \pm 6.1 \mathrm{~s}\right.$ for the $\mathrm{ATGL}^{-/-}$and $58.1 \pm 5.8 \mathrm{~s}$ for the WT), which means that overall muscular mitochondrial oxidative capacity was comparable between the genotypes. Despite similar in vivo mitochondrial oxidative capacities, the electro-stimulated muscles from $\mathrm{ATGL}^{-1}$ mice displayed significantly lower force production and increased muscle relaxation time than the WT. These findings suggest that other mechanisms than mitochondrial dysfunction cause the impaired muscle performance of $\mathrm{ATGL}^{-1}$ mice. 


\section{Introduction:}

Adipose triglyceride lipase (ATGL), also known as desnutrin, is a ubiquitous lipolytic enzyme, mainly expressed in hepatocytes, testes, adipocytes and myocytes ${ }^{29,33,38,51,54,58}$. This lipase is highly specific for triglycerides (TG) and catalyzes the first step of its hydrolysis ${ }^{58}$. Wholebody ATGL knockout mice ( $\mathrm{ATGL}^{-1}$ ) are mildly obese and accumulate lipids in various tissues such as skeletal muscle and heart, and ultimately develop lethal cardiomyopathy ${ }^{16}$. The ATGL 1- mice have significantly reduced plasma free fatty acids (FFA), improved insulin sensitivity and glucose tolerance evidencing a reliance on oxidation of glucose instead of fatty acid (FA) to produce energy $16,19,30,41$.

Fatty acids are the major energy substrates for muscle under submaximal exercise. Thus, diminished FA availability is one of the explanations for the exercise intolerance and poor endurance capacity of the $\mathrm{ATGL}^{-/}$mice ${ }^{19,47}$. Reduced exercise performance could also be explained by the cardiac dysfunction that the $\mathrm{ATGL}^{-1-}$ mice present at older age ${ }^{19}$. However, young $\mathrm{ATGL}^{-1-}$ mice without cardiac lipid accumulation, and adult $\mathrm{ATGL}^{-1-}$ mice with a normal cardiac ATGL expression, still present abnormal exercise performance ${ }^{19,47}$. These studies show that even with a normal cardiac function, $\mathrm{ATGL}^{-} \%$ mice remain exercise intolerant.

While whole-body ATGL ablation causes tissue-specific adaptations ${ }^{30}$, the most pronounced modulation of biological processes by ATGL occurs in cardiac muscle. In this tissue, genetic ablation of ATGL results in a down-regulation of the transcripts associated with oxidative pathways, namely genes involved in fatty acid uptake and mitochondrial $\beta$-oxidation, as well as in glycolysis and tricarboxylic acid cycle (TCA ${ }^{42}$. Furthermore, a recent study demonstrated that mitochondrial substrate oxidation and respiration is severely disrupted in cardiac muscle of $\mathrm{ATGL}^{-1-}$ mice, due to PPAR signalling ${ }^{17}$.

Although modulation of skeletal muscle metabolism by ATGL is not as prominent as in cardiac muscle, down-regulation of genes involved in ATP biosynthesis has been reported for $\mathrm{ATGL}^{-1}$ skeletal muscle ${ }^{42}$. Moreover, the accumulation of intramyocellular lipids (IMCL) in skeletal muscle might be associated with mitochondrial dysfunction as in obese or type 2 diabetic patients ${ }^{25,39,40}$ through a mechanism not fully understood but probably involving the accumulation of active lipid intermediates along with IMCL deposition, deficits in mitochondrial $\beta$-oxidation and increased formation of reactive oxygen species ${ }^{44,46,48}$. Whether the IMCL accumulation affects skeletal muscle mitochondrial function in $\mathrm{ATGL}^{-1}$ mice is unknown.

Taken together, these data suggest that the compromised exercise performance of ATGL mice is not solely explained by the inability to generate FFA for muscle oxidation, but could be associated with reduced mitochondrial oxidative capacity. We, therefore, hypothesized 
that $\mathrm{ATGL}^{-1-}$ mice have impaired muscular mitochondrial function, which contributes to their poor exercise tolerance.

To test this hypothesis, we evaluated in vivo and non-invasively the extent of IMCL accumulation in the skeletal muscle of $\mathrm{ATGL}^{-/}$-mice using ${ }^{1} \mathrm{H}$ magnetic resonance spectroscopy (MRS). To assess mitochondrial oxidative capacity of $\mathrm{ATGL}^{-1-}$ mice in vivo, gated dynamic ${ }^{31} \mathrm{P}$ MRS of electro-stimulated muscles was recorded. These parameters were correlated with force production and post-contraction relaxation time of $\mathrm{ATGL}^{-/-}$muscle. 


\section{Materials and Methods:}

\section{Animals:}

All the experimental procedures were approved by the Radboud University Nijmegen Medical Centre Animal Ethics Committee, Nijmegen, the Netherlands.

$\mathrm{ATGL}^{-/-}$mice, obtained by targeted homologous recombination as described before ${ }^{16}$, were a kind gift from R. Zechner, Institute of Molecular Biosciences, University of Graz, Austria. $\mathrm{ATGL}^{-}$and wild type (WT) littermate mice were generated by breeding heterozygous mice for the deleted allele. Male ATGL $\%(8 \pm 1 \mathrm{wk})$ and WT control mice $(10 \pm 2 \mathrm{wk})$ used in this study were housed under a light-dark cycle of 12:12 h with controlled temperature (22-24 $\left.{ }^{\circ} \mathrm{C}\right)$. Animals had permanent ad libitum access to mouse standard chow and water.

\section{MRS studies}

All in vivo MR studies were performed on a 7 Tesla horizontal magnet interfaced with a clinical console (Clinscan, Bruker Biospin, Ettlingen Germany). During surgical procedures and MR measurements mice were anaesthetized with 1-2 \% isoflurane (Pharmachemie BV, Haarlem, Netherlands) in a gas mixture of 50:50 \% $\mathrm{O}_{2} / \mathrm{N}_{2} \mathrm{O}$ delivered through a face mask. Body temperature was maintained at $37{ }^{\circ} \mathrm{C}$ using heating pads and monitored rectally with a fluoroptic thermometer (Luxtron 712, Santa Clara, California, USA). Mouse breathing was monitored in the scanner using a pressure sensor recording thorax movement (SA Instruments Inc, New York, USA).

Intramyocellular lipid levels were measured in vivo in the tibialis anterior (TA) of $7 \mathrm{ATGL}^{\%}$ and 9 WT mice by single voxel ${ }^{1} \mathrm{H}$ MRS, as previously described ${ }^{15}$ with minor adaptations. Briefly, mice were anesthetized and their left-leg positioned under a four-element array ${ }^{1} \mathrm{H}$ surface coil, in order to align the TA muscle fibres with the magnetic field, which provides optimal spectral separation between the resonances of extra and intramyocellular lipids. Anatomical reference images were taken using a spin-echo pulse sequence (repetition time (TR) of 2500 ms and echo-time (TE) of $30 \mathrm{~ms}$ ) for careful positioning of a $3.1 \times 1 \times 1 \mathrm{~mm}^{3}$ voxel within the TA. Automatic first- and second-order shimming was performed using the FASTMAP method in the same voxel. The ${ }^{1} \mathrm{H}$ MR spectra were acquired using a PRESS sequence with additional six outer volume suppression bands and the following parameters: TR/TE= 1500/16 ms and 1024 averages. Two ${ }^{1} \mathrm{H}$ spectra were acquired, one without and the other with water suppression which was achieved using the VAPOR method.

Skeletal muscle mitochondrial oxidative capacity was assessed in vivo in $9 \mathrm{ATGL}^{-1-}$ and $10 \mathrm{WT}$ mice using ${ }^{31} \mathrm{P}$ MRS. Contraction of the calf muscle complex, i.e. gastrocnemius (GAST) and soleus (SOL) muscles, was achieved by electro-stimulation of the sciatic nerve as described 
previously ${ }^{24}$. After anaesthesia, a minor incision was made in the mouse hip and the sciatic nerve exposed. To avoid contraction of the dorsal flexors, the peroneal nerve was severed ${ }^{43}$. An electrode (tensile flex UT3607TF) was tunnelled through the upper leg and connected to the sciatic nerve by stitching the electrode to the surrounding tissue leaving the nerve in its natural position. A second electrode was fed through the skin near the Achilles tendon, functioning as ground reference for the stimulation pulses. The mouse was positioned in the setup in its prone position and the hind-leg was placed on top of the MR compatible force transducer by fixing the foot in a close fitting cradle and the knee into a cavity in a Perspex plate at a 90ㅇ angle. Optimal stimulation current was determined by varying the current (1.25-2.5mA) to elicit isometric tetanic contractions, with a pulse train of 250 ms duration and stimulus frequency of $150 \mathrm{~Hz}$, yielding the maximal force. Stimulation of the calf muscle complex occurred every $3 \mathrm{~s}$ over one minute, (muscle duty cycle of $8 \%$ ). The stimulation frequency of $150 \mathrm{~Hz}$ was chosen to generate fully fused isometric tetani at maximum force as determined by frequency-force relationship in SOL and GAST of WT mice ${ }^{22,23}$.

A dual coil setup consisting of a ${ }^{31} \mathrm{P}$ solenoid coil $(121.5 \mathrm{MHz})$ was used for unlocalized ${ }^{31} \mathrm{P}$ MRS and a ${ }^{1} \mathrm{H}$ surface coil $(300.2 \mathrm{MHz})$ used for reference MR imaging and localized shimming of the hind-leg. A user interface was designed to control the stimulation, measure force transduction and synchronize the measurements to the timing of the pulse sequences using LabView.

Initially, one ${ }^{31} \mathrm{P} M R$ spectrum was acquired (TR $=10 \mathrm{~s}$, number of points $=2048$, spectral width of $5 \mathrm{kHz}, 64$ averages) with the mouse leg at rest. Subsequently, eight-time series of ${ }^{31} \mathrm{P}$ MR spectra were acquired before ( $T R=10 \mathrm{~s}, 10 \mathrm{scans}$ ), during (TR=9 s, 6 scans) and after ( $T R=10 \mathrm{~s}, 30$ scans) the muscle contractions. Isometric tetanic contractions of the muscle $(250 \mathrm{~ms}, 150 \mathrm{~Hz}$ ) occurred every $3 \mathrm{~s}$ over a period of one minute, summing 20 contractions per cycle.

After the electro-stimulation protocol, samples of contracted and resting contralateral hindlimb were freeze-clamped and immediately stored in liquid nitrogen. Additionally, blood was withdrawn from the hepatic vein, centrifuged and plasma recovered from centrifugation 10 min, $1000 \mathrm{~g}$. Tissue and plasma samples were stored at $-80^{\circ} \mathrm{C}$ until further analysis.

\section{Data processing of in vivo studies}

All ${ }^{1} \mathrm{H}$ and ${ }^{31} \mathrm{P}$ MR spectra were fitted in the time domain by using AMARES ${ }^{53}$ in the jMRUI software package ${ }^{36}$. The IMCL level in the TA muscle was calculated from the lipid methylene peak area $(1.33 \mathrm{ppm})$ and was expressed relatively to the total creatine (tCr) methyl peak area (3.02 ppm). 
The eight-time series of forty-six ${ }^{31} \mathrm{P} M R$ spectra were added retrospectively, and peak areas of inorganic phosphate (Pi), phosphocreatine (PCr), $\gamma^{-}, \alpha$ - and $\beta$-ATP signals were fitted by Gaussian lineshapes. Absolute concentrations of the phosphorus-metabolites were calculated assuming that at rest the mouse muscle have $7.8 \mathrm{mM}$ of ATP ${ }^{20}$. Intracellular $\mathrm{pH}$ was calculated from the chemical shift difference between $\mathrm{Pi}$ and $\mathrm{PCr}$ resonances ${ }^{50}$. The recovery of $\mathrm{PCr}$ after the contractions was fitted with a mono-exponential function (Graphpad 5.03, La Jolla, CA, USA). The $\mathrm{TPCr}$ value is the in vivo measure of skeletal muscle mitochondrial oxidative capacity.

Signals derived from the force transducer were digitized to a sampling frequency of $50 \mathrm{kHz}$ and analyzed with Matlab (Mathworks Inc, Natick, USA), where each contraction was filtered and peak force and half relaxation time (HRT, the time in which the force falls from half to a quarter of the maximal value of each contraction ${ }^{11,12}$ ) were determined. Force production was normalized to the cross-sectional area (CSA) of the muscle, determined from the hindlimb axial images obtained prior to the electro-stimulation protocol. CSA were determined for the entire hind-limb muscle region by delineating it from the slices where all animals had similar tibial bone area $\left(1.4 \pm 0.1 \mathrm{~mm}^{2}\right)$ using Image J software (NIH Image version 1.44, http://rsbweb.nih.gov/ij/).

\section{Skeletal muscle electron-microscopy}

Ultrastructural morphology was examined using transmission electron-microscopy. Muscle tissue sections from extensor digitorum longus (EDL) and the SOL were fixed in $2.5 \%$ glutaraldehyde in $0.1 \mathrm{M}$ phosphate buffer ( $\mathrm{pH}$ 7.4). Post-fixation was performed in $1 \%$ osmium tetroxide in $0.1 \mathrm{M}$ cacodylate buffer $(\mathrm{pH} 7.4)$ supplemented with $1.5 \%$ potassium ferrocyanide. The samples were then dehydrated and embedded in epon. Ultrathin sections were examined using a Philips CM100 electron-microscope. Lipid droplet (LD) diameter and content was determined in EDL and SOL using image J software.

\section{Fibre typing of tibialis anterior}

The fibre type composition of TA was determined by immunohistochemical analyses in serial cross-cryosections of the muscle, carefully mounted in uncoated glass slides. The relative proportion of type I and type II fibres was determined with specific antibody against myosin heavy chain type I (A4.840, Developmental Studies Hybridoma Bank, diluted 1:25 in PBS) combined with Alexa555- conjugated secondary antibody.

Slides were analysed using a fluorescence microscope (Model E800; Nikon Instruments Europe, Badhoevedorp, the Netherlands) coupled with a progressive scan colour CCD camera with a (Basler A113 C) colour filter (Bayer). Epifluorescence signal was recorded 
using a fluorescein isothiocyanate excitation filter $(465-495 \mathrm{~nm})$ for muscle fibre-type and a 4',6-diamino-2-phenylindole UV excitation filter (340-380 nm) for laminin.

Digital images were captured with a magnification of 120 from at least 6 different fields of view per muscle section and processed with Lucia v4.81 software.

\section{Intraperitoneal glucose tolerance test (ipGTT)}

A week before the MRS measurements, mice were fasted for $6 \mathrm{~h}$ and injected intraperitoneally with a bolus of glucose $\left(1.5 \mathrm{~g} \cdot \mathrm{kg}^{-1}\right)$. Blood glucose levels were monitored before, and 15, 30, 60, 90 and 120 min after injection with an Accu-Chek performa glucometer (Roche, Manheim, Germany).

\section{Resting and contraction induced-metabolite depletion}

Absolute tissue concentrations of high-energy phosphate compounds were determined in resting muscle from ATGL ${ }^{-1-}$ and WT mice as described before ${ }^{57}$. Briefly, muscles were rapidly excised and freeze clamped, followed by extraction with $1.3 \mathrm{M}$ perchloric acid and neutralization with $1 \mathrm{M}$ potassium bicarbonate. Thereafter, tissue contents of $\mathrm{PCr}$, free $\mathrm{Cr}$ and ATP were determined by high-performance liquid chromatography.

To determine the contraction cost in muscle glycogen content, freeze-clamped samples of contracted and contralateral resting hind-limbs were first powdered under a liquid-nitrogen atmosphere. Muscle tissue was then digested with two volumes of potassium hydroxide 30 $\%(\mathrm{w} / \mathrm{v})$ at $70^{\circ} \mathrm{C}$ for $30 \mathrm{~min}$. The precipitation of glycogen was achieved by adding $1 \mathrm{~mL}$ of $6 \%$ $(\mathrm{w} / \mathrm{v})$ sodium sulphate and $6 \mathrm{~mL}$ of $99.9 \%$ ethanol to the digested tissue. Samples were kept overnight at $4 \mathrm{C}$. The samples were then centrifuged, the supernatant was discarded and the pellet resuspended in $50 \mathrm{mM}$ sodium acetate $\mathrm{pH}$ 4.5. Sixteen units of amyloglucosidase from Aspergillus niger (Sigma-Aldrich, Steinheim, Germany) were added to hydrolyse the glycogen into glucose monomers. Samples were incubated overnight at $55^{\circ} \mathrm{C}$ and $\mathrm{pH}$ was then readjusted to 7. Glucose monomers were quantified using an assay kit (Invitrogen, Breda, Netherlands). Glycogen levels were expressed in glucosyl units.g of wet weight ${ }^{-1}$. Plasma levels of glucose, lactate and FFA were determined from mice that underwent the electro-stimulation protocol, using enzymatic colorimetric assays (Invitrogen, Breda, Netherlands, Eton Bioscience, Durham, NC USA, Wako HR (2), Wako Diagnostics, Neuss, Germany). Triglyceride levels were determined using a clinical Cardiocheck analyzer (Polymer Technology Systems, Indianapolis, USA). 


\section{Statistical analysis}

Statistical analyses were performed using GraphPad prism 5.03 (GraphPad Software, La Jolla, CA, USA). Differences between genotypes were evaluated applying unpaired Student's t-test or two-way ANOVA with a Bonferroni post hoc test as appropriate. Statistical significance was set at $p<0.05$. 


\section{Results:}

Relevant characteristics of ATGL ${ }^{-1}$ and WT mice are presented in table 1 . The average body weight of $\mathrm{ATGL}^{-/}$mice was higher than their WT littermates $(p<0.001)$. Plasma glucose levels were similar between the genotypes at fed state and after $6 \mathrm{~h}$ of fasting. The glucose tolerant phenotype of the ATGL $\%$ mice was confirmed by a glucose challenge showing lower plasma glucose levels at $15 \mathrm{~min}\left(11.7 \pm 1.0 \mathrm{mM}\right.$ for $\mathrm{ATGL}^{-/}$vs. $14.4 \pm 0.9 \mathrm{mM}$ for WT mice) and $60 \mathrm{~min}$

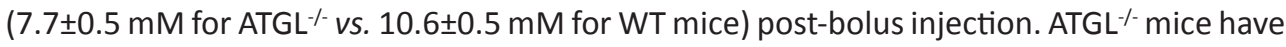
also lower ipGTT area under the curve (AUC), $p<0.01$.

\section{IMCL content and fibre type composition}

In order to evaluate lipid accumulation in $\mathrm{ATGL}^{-1-}$ muscles, the EDL and the SOL muscles were analysed for IMCL content by electron-microscopy (Fig. 1A). Lipid droplets were larger and more numerous in the SOL muscle (mainly comprised of red oxidative fibres) of $\mathrm{ATGL}^{-1}$ mice compared to WT mice. This observation, albeit less pronounced, was also valid for the EDL muscle which have a more glycolytic phenotype (Fig.1B). Interestingly, it was observed that in $\mathrm{ATGL}^{-1}$ muscles neighboring fibres have profound differences in LD content and size: fibres with higher mitochondrial content and thicker Z-lines (most likely representing the more oxidative fibres in the EDL) had abundant and fairly large LD, in contrast to fibres with a low mitochondrial content and thinner Z-lines (characteristic of glycolytic fibres). In both muscles from the $\mathrm{ATGL}^{-1-}$ mice, signs of autophagy were detectable, as well as crystal-like

Table 1: Characteristics of ATGL $\%(n=14)$ and WT mice $(n=16)$.

\begin{tabular}{lcc}
\hline & ATGL $\%$ & WT \\
\hline Age (weeks) & $8 \pm 1$ & $10 \pm 2$ \\
Body weight (g) & $25.8 \pm 0.3^{* * *}$ & $23.0 \pm 0.4$ \\
Fed glycaemia (mM) & $8.2 \pm 0.6$ & $8.9 \pm 0.5$ \\
6h Fasted glycaemia (mM) & $7.4 \pm 0.5$ & $7.7 \pm 0.5$ \\
IpGTT AUC (mM·min) & $1067 \pm 54^{* *}$ & $1277 \pm 56$ \\
\hline
\end{tabular}

Values are presented as mean $\pm S D$, statistical differences indicated with Student $t$-test for $\mathrm{p}<0.05 ;{ }^{* *}$ and $* * *$ significantly different from WT $p<0.01$ and $p<0.001$. IpGTT, Intraperitoneal glucose tolerance test; AUC, represents the total area under the curve. 
Figure 1: Assessment of lipid accumulation in EDL and SOL muscles of ATGL-/- and WT mice.

A

EDL

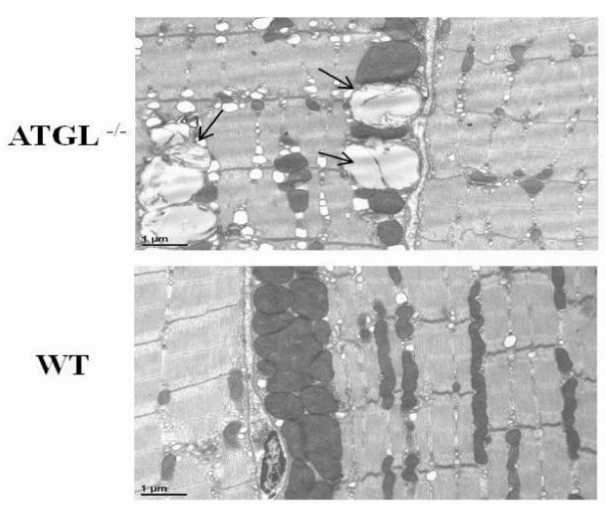

SOL
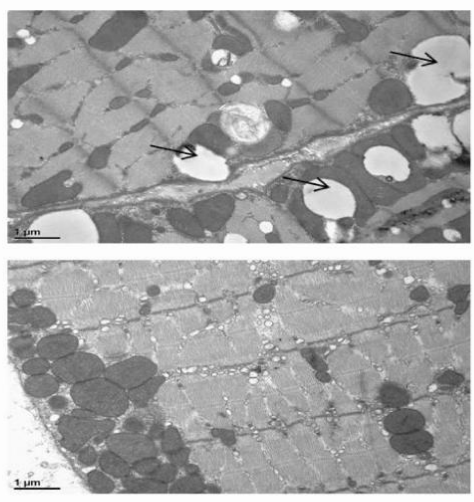

1 im
B
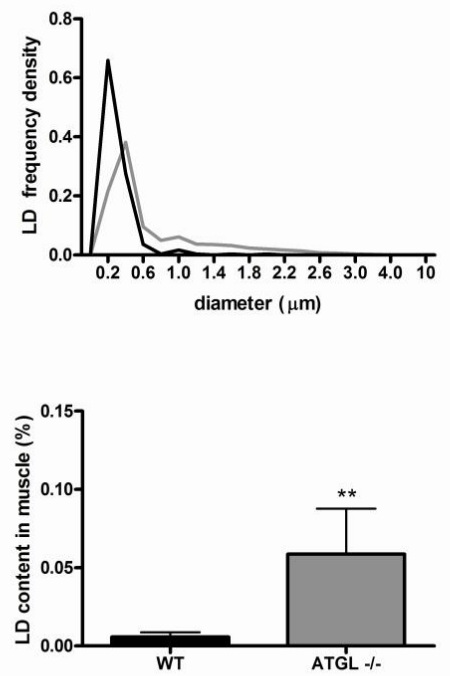

SOL
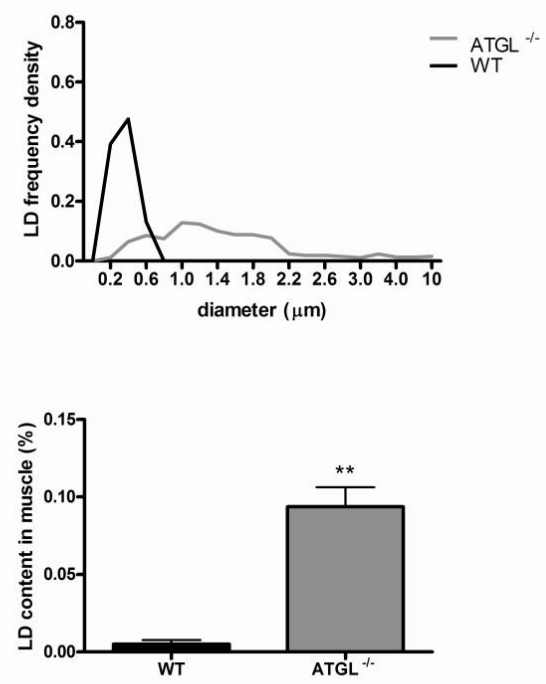

A. Representative electron-microscopy images of extensor digitorum longus (EDL) and soleus muscle (SOL) of ATGL 1- and WT mice. Note the higher amount of lipid droplets (LD) (arrows) in muscles of $\mathrm{ATGL}^{-\%}$ mice and its different distribution among neighbouring fibres. B. LD frequency density and LD content in EDL and SOL muscles of ATGL 1- and WT mice ( $\mathrm{n}=2$ for both groups). Values presented are means $\pm S E M$, statistical differences indicated with Student $t$-test for $\mathrm{p}<0.05$; ** significantly different from WT $\mathrm{p}<0.01$. 
Figure 2: IMCL content in TA of ATGL-/- and WT mice.

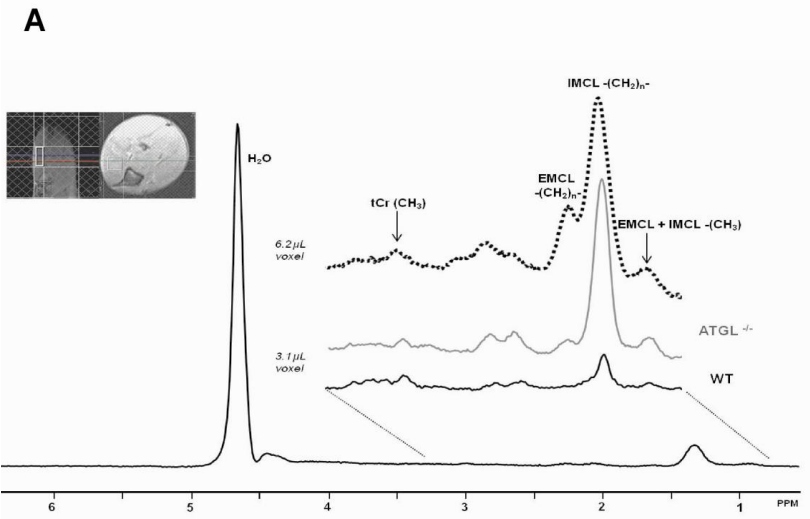

B

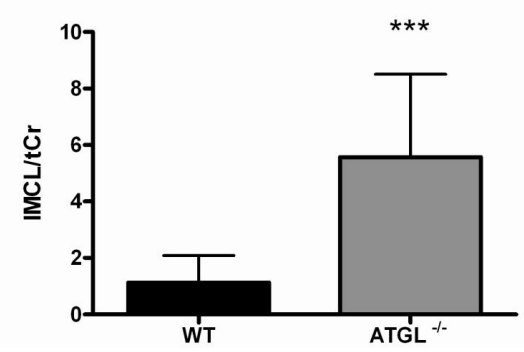

A. representative single-voxel $(3.1 \mu \mathrm{L}){ }^{1} \mathrm{H}$ MR spectrum measured in TA of $\mathrm{ATGL}^{-1}$ and WT mice. Note in ${ }^{1} \mathrm{H} M R$ spectrum acquired with a $6.2 \mu \mathrm{L}$ voxel, the presence of both $\mathrm{EMCL}$ and IMCL methylene peaks; B. Spectral quantification of IMCL in TA of $n=9$ WT (black bar) and $n=7 A_{T G L} \%$ (grey bar) mice. Values are presented as mean $\pm S D$, statistical differences indicated with Student $t$-test for $p<0.05$. $* * *$ Significant difference $p=0.0007$ vs. WT.

structures in the LD. Despite the aberrant lipid deposition in skeletal muscle of $\mathrm{ATGL}^{-1}$ mice, no abnormal features were observed in the mitochondrial morphology or in the structure of the contractile filaments.

The IMCL pool was also assessed in vivo in TA muscle of $\mathrm{ATGL}^{-1-}$ and $\mathrm{WT}$ mice by ${ }^{1} \mathrm{H}$ MRS. This muscle was chosen due to its natural alignment with the magnetic field, a pre-requisite to resolve the resonances of extra- and intramyocellular lipids ${ }^{3,4}$. To quantify these two lipid pools with localized ${ }^{1} \mathrm{H}$ MRS, a small voxel $(3.1 \mu \mathrm{L})$ was positioned inside of the TA muscle. In the spectra of all mice, $\mathrm{ATGL}^{-/}$and $\mathrm{WT},{ }^{1} \mathrm{H}$ resonances of only $\mathrm{IMCL}$ were visible in the methylene and methyl spectral region. Signals of extramyocellular lipids were detected when larger voxels were applied (6.2 $\mu \mathrm{L}$ ) (see Fig. $2 \mathrm{~A}$ ). In ${ }^{1} \mathrm{H}$ MR spectra of ATGL ${ }^{-1-}$ mice, the height of the IMCL peak representing the methylene protons (1.33 ppm) was substantially 
increased compared to that of the WT mice (Fig. 2A). IMCL content in TA was normalized for $\mathrm{tCr}$ (Fig. 2B) and this ratio was $\sim 6$ times higher in TA of $\mathrm{ATGL}^{-1-}$ mice than the TA of WT mice (5.6 \pm 2.9 vs. $1.1 \pm 0.9, \mathrm{p}=0.0007)$.

Changes in muscle fibre type composition can occur in response to skeletal muscle lipid surplus $^{49}$. However, this was not observed in the TA muscle of $\mathrm{ATGL}^{-1-}$ mice. Irrespective of genotypes, the TA maintained its major composition of type II (fast-twitch) fibres (Fig. 3A). TA muscle from ATGL $\mathrm{T}^{-1}$ mice was composed of $81.1 \pm 4.8 \%$ vs. $18.9 \pm 4.8 \%$ type II and type I fibres compared to $79.6 \pm 5.8 \%$ vs. $20.4 \pm 5.8 \%$ type II and type I fibres in WT (Fig. 3B).

Figure 3: Fibre typing of tibialis anterior muscle in ATGL-/- and WT mice.

A
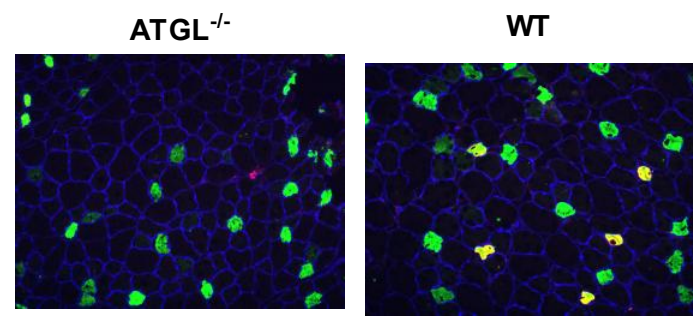

B

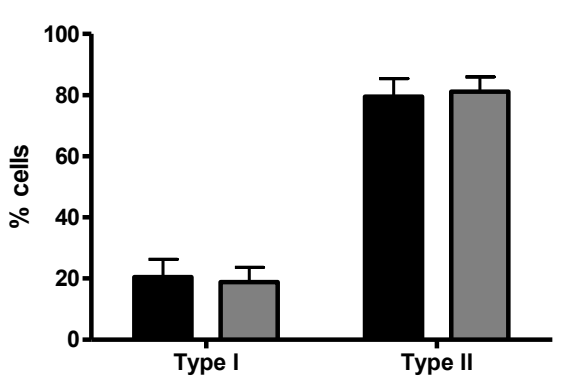

A. Tibialis anterior cross-sectional area of ATGL $\%$ and WT stained for myosin heavy chain I. Type I fibres (green) and type II fibres (not coloured), laminin (blue). B. Quantification of type I and type II fibres in TA given in \% of cells ( $n=7$ for both groups). Values presented are means \pm SD.

\section{Energy state of the resting skeletal muscle}

The ${ }^{31} \mathrm{P}$ MR spectra acquired in the resting muscle of WT mice showed resonances assigned to $\mathrm{Pi}, \mathrm{PCr}$ and ATP (Fig. 4). Resting muscles of $\mathrm{ATGL}^{-1}$ and $\mathrm{WT}$ mice had similar tissue concentrations of $\mathrm{PCr}$ and $\mathrm{Pi}$, comparable ratios of $\mathrm{PCr} / \mathrm{Pi}$ and $\mathrm{PCr} / \mathrm{ATP}$ and cellular $\mathrm{pH}$ (see table 2).

In line with the in vivo ${ }^{31} \mathrm{P}$ MRS data, the biochemical determinations of muscle highenergy metabolites showed no significant differences between the groups. The average ATP concentration in freeze-clamped muscles was $28.7 \pm 5.0 \mu \mathrm{mol} \cdot \mathrm{g}$ dry weight ${ }^{-1}$ for $\mathrm{ATGL}^{-1-}$ mice and 33.7 $\pm 2.4 \mu \mathrm{mol} \cdot g$ dry weight ${ }^{-1}$ for WT mice $(p=0.17)$. PCr concentration was $66.2 \pm 10.7$ $\mu \mathrm{mol} \cdot \mathrm{g}$ dry weight ${ }^{-1}$ for $\mathrm{ATGL}^{-/}$mice and $75.8 \pm 17.6 \mu \mathrm{mol} \cdot \mathrm{g}$ dry weight ${ }^{-1}$ for WT mice $(p=0.40)$. 
Figure 4: ${ }^{31}$ P MR spectroscopy of resting skeletal muscle.

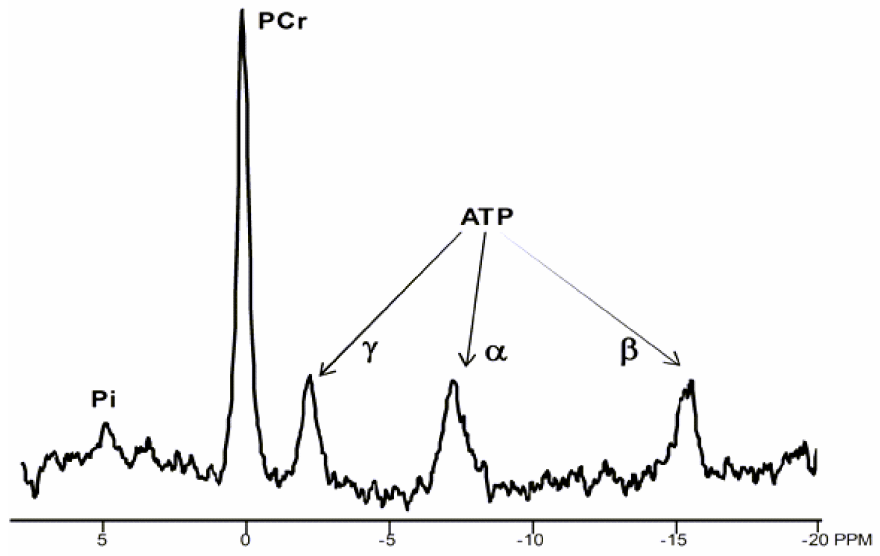

${ }^{31} \mathrm{P}$ MR spectrum measured in the resting hind-limb of a WT mouse, with 64 averages, TR of 10s, and exponential filter applied. Pi, inorganic phosphate, PCr, phosphocreatine, and ATP, adenosine triphosphate $(\gamma, \alpha, \beta)$.

Table 2: Metabolite concentrations and $\mathrm{pH}$ calculated from ${ }^{31} \mathrm{P} M \mathrm{R}$ spectra acquired at rest and after muscle stimulation of ATGL $\%(n=9)$ and WT $(n=10)$ mice.

\begin{tabular}{|c|c|c|c|c|}
\hline & \multicolumn{2}{|c|}{$\mathrm{ATGL}^{-1-}$} & \multicolumn{2}{|c|}{ WT } \\
\hline & Rest & End stimulation & Rest & End stimulation \\
\hline [PCr], mM & $25.6 \pm 7.6$ & $13.2 \pm 6.6$ & $20.1 \pm 6.1$ & $11.8 \pm 4.2$ \\
\hline$\left[\mathrm{P}_{\mathrm{i}}\right], \mathrm{mM}$ & $3.5 \pm 1.7$ & $15.2 \pm 4.6$ & $2.6 \pm 1.9$ & $15.1 \pm 8.2$ \\
\hline$[\mathrm{PCr}] /\left[\mathrm{P}_{\mathrm{i}}\right]$ & $11.4 \pm 10.5$ & $0.9 \pm 0.5$ & $11.1 \pm 9.9$ & $1.1 \pm 0.8$ \\
\hline$[\mathrm{PCr}] /[\gamma \mathrm{ATP}]$ & $3.2 \pm 1.0$ & $2.0 \pm 1.1$ & $2.6 \pm 0.8$ & $1.5 \pm 0.9$ \\
\hline $\mathrm{pH}$ & $7.14 \pm 0.15$ & $6.94 \pm 0.06$ & $7.11 \pm 0.10$ & $7.00 \pm 0.09$ \\
\hline
\end{tabular}

Values are presented as mean $\pm \mathrm{SD}$. $\mathrm{PCr}$, phosphocreatine; $\mathrm{Pi}$, inorganic phosphate; ATP, adenosine triphosphate. Values are presented as mean $\pm \mathrm{SD}$,

\section{Muscle performance of $\mathrm{ATGL}^{-/-}$and WT mice upon electro-stimulation}

Mitochondrial oxidative capacity and muscular performance of $\mathrm{ATGL}^{-1-}$ mice were evaluated in a combined electro-stimulation protocol based on in vivo muscle electro-stimulation with gated dynamic ${ }^{31} \mathrm{P} \mathrm{MRS}^{24}$.

Electro-stimulation through the sciatic nerve at a frequency of $150 \mathrm{~Hz}$ for $250 \mathrm{~ms}$ resulted in fully fused isometric tetanic contractions of the calf-muscle complex in both genotypes, $\mathrm{ATGL}^{-}$and WT mice (see fig. 5A). The stimulation current applied to achieve maximal force in $\mathrm{ATGL}^{-1-}$ muscles was $1.72 \pm 0.03 \mathrm{~mA}$, significantly lower than the $2.00 \pm 0.06 \mathrm{~mA}$ applied to the WT muscles, $(p<0.001)$. This optimal current was then used to stimulate the calf-muscle 
complex to contract every 3 seconds over one minute $(0.33 \mathrm{~Hz})$. The force produced in the first twitch of the electro-stimulation train was substantially lower in $\mathrm{ATGL}^{-1-}$ mice than in the WT mice $\left(0.016 \pm 0.001 \mathrm{~N} \cdot \mathrm{mm}^{-2}\right.$ vs. $0.030 \pm 0.005 \mathrm{~N} \cdot \mathrm{mm}^{-2}, \mathrm{p}<0.0001$, Fig. $\left.5 B\right)$. Over the 20 isometric contractions, the force produced by the $\mathrm{ATGL}^{-}$mice was persistently lower than that of the WT mice (Fig. 5C). However, the $\mathrm{ATGL}^{-1-}$ mice had a force decay of $29 \pm 1 \%$ between the first and the last contraction of the stimulation-train, similar to the decay of $27 \pm 2 \%$ for the WT mice. To test whether differences in force production were due to the different electro-stimulation currents applied, ATGL ${ }^{-/-}$and WT mice $(n=5)$ were subjected to the same optimal stimulation current of $1.92 \mathrm{~mA}$. Also under these conditions ATGL $\%$ mice produced lower force than the WT mice (Fig. 5D).

Figure 5: Muscle contraction profile of ATGL $\%$ and WT mice.

A

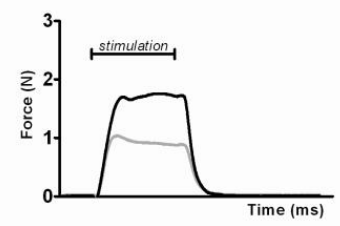

D

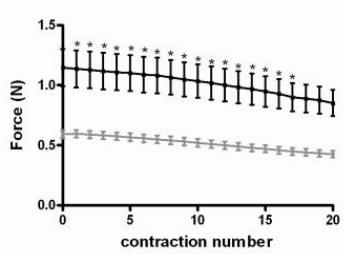

B

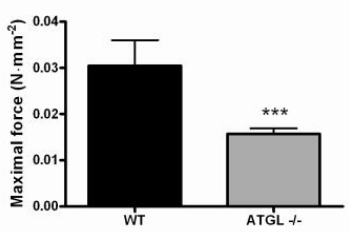

E

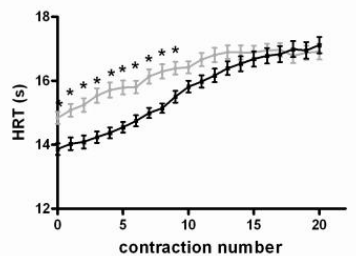

C

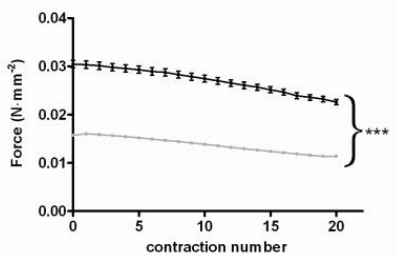

A. example of a fully fused isometric tetanic contraction of calf-muscles in ATGL $\%$ (grey) and WT (black) achieved by electro-stimulation during $250 \mathrm{~ms}$, at $150 \mathrm{~Hz}$ of stimulation frequency. B. Maximal force produced upon the first muscle twitch of ATGL ${ }^{--}$mice ( $n=9$; grey bar) and WT mice ( $n=10$; black bar). Muscle contraction was achieved by optimal electro-stimulation of calf-muscles in $\mathrm{ATGL}^{-\%}$ mice at $1.72 \pm 0.03 \mathrm{~mA}$ and WT mice at $2.00 \pm 0.06 \mathrm{~mA}$. C. Force $\left(\mathrm{N} \cdot \mathrm{mm}^{-2}\right)$ produced in the electro-stimulation protocol with 20 isometric tetanic contractions. D. Absolute force produced by $\mathrm{ATGL}^{\%}$ and WT mice with a stimulation current of $1.92 \mathrm{~mA}$ ( $\mathrm{n}=5$ in both groups). E. Muscular halfrelaxation time (HRT) determined in each contraction of $\mathrm{ATGL}^{-\%}$ mice (grey dots) and WT mice (black dots). Values are presented as mean \pm SEM, statistical differences were assessed by a Student $t$-test (B) and two-way ANOVA with Bonferroni post-test (C, D and E). * and ${ }^{* * *}$ significant different from $\mathrm{WT}, \mathrm{p}<0.01$ and $\mathrm{p}<0.001$, respectively. 
The muscular relaxation time was determined by the HRT in each tetanic contraction, as the time that the force falls from half to a quarter of the maximal value. The HRT of the WT muscles describes a sigmoidal curve, and achieved a steady-state of relaxation only at the final stage of the stimulation train, around the $17^{\text {th }}$ contraction (see fig. 5E). The HRT of ATGL 1- muscles describes an exponential curve and during the first ten contractions these animals required more time to relax than the WT muscles $(p<0.01)$. Muscles from the knockout model achieved a stationary phase of relaxation after the $12^{\text {th }}$ contraction. In general, the percentage change of HRT between the first and the last contraction of the cycle was higher in WT mice (102 $\pm 1 \%$ to $125 \pm 3 \%)$ than in $\mathrm{ATGL}^{-/}$mice $(102 \pm 1 \%$ to $113 \pm 2 \%), \mathrm{p}<0.01$, which suggests that $\mathrm{ATGL}^{-/}$mice present muscular fatigue even at the onset of the electrostimulation protocol.

Although differences in muscular force production and HRT between $\mathrm{ATGL}^{-1}$ and WT mice could theoretically be attributed to muscle mass composition, the levels of $\mathrm{tCr}$ and muscle CSA discarded this possibility. The resting $\mathrm{ATGL}^{-}$and WT muscles had similar $\mathrm{tCr}$ contents as determined biochemically, with $49.2 \pm 3.5 \mu \mathrm{mol} \cdot \mathrm{g}$ dry weight ${ }^{-1} v s .52 .4 \pm 17.0 \mu \mathrm{mol} \cdot \mathrm{g}$ dry weight $^{-1}$, respectively. Similarly, the muscle CSA determined for the slices with the same tibial bone area, was $34.8 \pm 1.9 \mathrm{~mm}^{2}$ for $\mathrm{ATGL}^{-}$and $37.7 \pm 5.3 \mathrm{~mm}^{2}$ for WT mice $(p=0.16)$.

\section{Contraction-induced metabolic cost}

To meet the energy requirements of a working muscle, creatine kinase operates in the first phase of exercise hydrolysing $\mathrm{PCr}$ to $\mathrm{Cr}$ and rephosphorylating ADP to ATP. The electrostimulation of the calf-muscle complex depleted the $\mathrm{PCr}$ pool by $46 \pm 14 \%$ and $41 \pm 18 \%$ for $\mathrm{ATGL}^{-/}$and WT mice, respectively. For both genotypes, the experienced PCr depletion during each time-point of contraction was correlated with decreased force production. In the ATGL I- and in the WT mice these two parameters correlated positively, suggesting that lower PCr matched a lower force production (Pearson correlation of 0.76 and $p=0.04$ vs. 0.81 and $\mathrm{p}=0.02$, respectively for $\mathrm{ATGL}^{-1}$ and WT mice).

Upon $\mathrm{PCr}$ depletion, muscle glycogen becomes an important source of energy. In this study, we could not measure contraction-induced glycogen depletion directly. Instead, calf muscles from the contracted and contralateral resting hind-limb were analysed afterwards to compare glycogen content. In the contralateral resting hind-limb, glycogen levels were different between the genotypes (Fig. 6). The $\mathrm{ATGL}^{-1-}$ mice had $\sim 30 \%$ less glycogen content than their counterparts $(15.8 \pm 5.3$ vs. $22.2 \pm 6.9 \mu \mathrm{mol}$ of glucosyl units.g wet weight $\left.{ }^{-1}, \mathrm{p}<0.05\right)$. Independently of the genotype, the glycogen level in the electrostimulated muscles decreased $\sim 50 \%$ compared to contralateral non-stimulated muscles. Similar to the correlation observed between $\mathrm{PCr}$ levels and force production during the 
Figure 6: Contraction-mediated glycogen depletion.

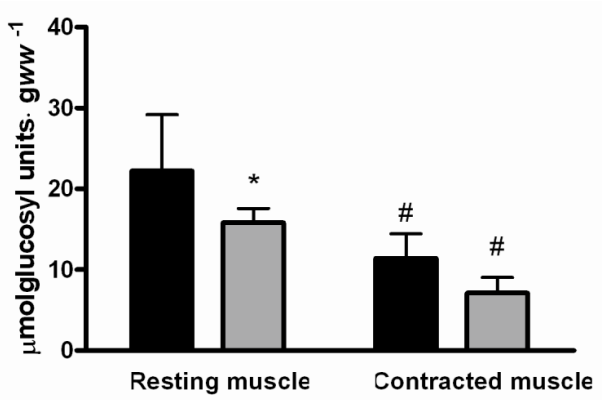

Glycogen content on the contracted and contralateral resting calf-muscles of $n=9 \mathrm{ATGL}^{-\%}$ (grey bars) and $n=8$ WT (black bars) mice. Values presented are mean \pm SD; statistical differences indicated with two-way ANOVA and Bonferroni post-test, * different from WT, $\mathrm{p}<0.05$, and \# different from the respective contralateral resting muscle, $p<0.001$.

electro-stimulation, the glycogen consumption, inferred from the glycogen variation from non-stimulated to stimulated muscles, was positively correlated with the change in force (Pearson correlation of 0.78 and $p=0.04$ vs. 0.83 and $p=0.01$, respectively for $\mathrm{ATGL}^{-/}$and WT mice).

After the muscle electro-stimulation protocol, WT $(n=5-8)$ and $\operatorname{ATGL}^{-/-}(n=7)$ mice had similar plasma lactate levels ( $3.7 \pm 1.0$ vs. $4.2 \pm 1.2 \mathrm{mM}$ respectively), and plasma glucose was mildly reduced in $\mathrm{ATGL}^{-1-}$ mice $5.8 \pm 2.7 \mathrm{mM}$ compared to WT mice $8.6 \pm 2.3 \mathrm{mM}$ ( $\left.p=0.09\right)$. The lipid profile of $\mathrm{ATGL}^{-1}$ mice showed a reduction in FFA $(0.42 \pm 0.13 \mathrm{mM})$ compared to WT mice $(0.73 \pm 0.30 \mathrm{mM}, \mathrm{p}=0.03)$, whereas TG levels were similar between the genotypes $(0.98 \pm 0.12$ $\mathrm{mM}$ vs. $1.24 \pm 0.52 \mathrm{mM}, \mathrm{p}=0.12$ ).

\section{In vivo muscular mitochondrial oxidative capacity}

Gated ${ }^{31} \mathrm{P}$ MRS was used to determine in vivo muscular mitochondrial oxidative capacity of $\mathrm{ATGL}^{-1-}$ mice. Figure 7A shows a sequence of ${ }^{31} \mathrm{P}$ MRS spectra acquired before, during and after the electro-stimulation of the calf-muscle complex in one $\mathrm{ATGL}^{-1}$ mouse. Upon stimulation, $\mathrm{PCr}$ is dephosphorylated to $\mathrm{Pi}$ and free $\mathrm{Cr}$ (not visible in ${ }^{31} \mathrm{P}$ spectra), and when stimulation ceases $\mathrm{PCr}$ is resynthesized to basal levels reflecting mitochondrial oxidative phosphorylation (Fig. 7B). Thus the resynthesis of $\mathrm{PCr}$ is an in vivo measure for mitochondrial oxidative capacity. Electro-stimulation of calf-muscle complex induced a $\mathrm{PCr}$ depletion of $\sim 43 \%$, and a $\sim 5$ fold increase in Pi levels, in both $\mathrm{ATGL}^{-\%}$ and WT mice (Table 2). Without significant changes in ATP amplitude during the muscle stimulation, the PCr/ATP ratio decreased similarly to $\mathrm{PCr}$ concentration, i.e. $\sim 42 \%$ for both groups. In addition, the 
intracellular $\mathrm{pH}$ of the electro-stimulated muscles from $\mathrm{ATGL}^{-}$and $\mathrm{WT}$ mice experienced similar reductions.

After the contraction block, muscle $\mathrm{PCr}$ pool was resynthesized to its initial levels. The fitting of the $\mathrm{PCr}$ recovery curves was made with a mono-exponential function (Fig. 7B) and retrieved the recovery time values graphically expressed in Fig. 7C. The mean value of $\mathrm{PCr}$ recovery time, $\tau \mathrm{TPC}$, was $54.1 \pm 6.1 \mathrm{~s}$ for the $\mathrm{ATGL}^{-/-}$mice and $58.1 \pm 5.8 \mathrm{~s}$ for the WT mice. From these $\tau \mathrm{PCr}$ values, we conclude that under the experimental conditions applied, $\mathrm{ATGL}^{-1-}$ and WT mice have comparable mitochondrial oxidative capacities.

Figure 7: In vivo skeletal muscle mitochondrial oxidative capacity of ATGL $\%$ and WT mice.

A

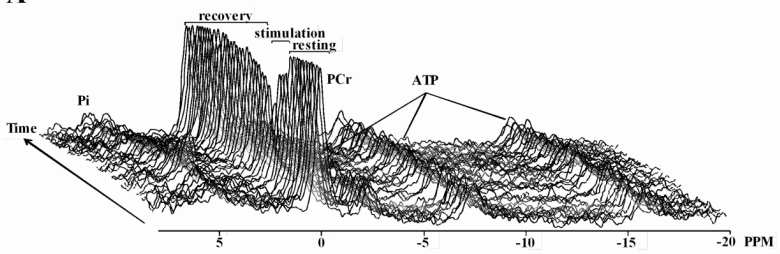

C

B
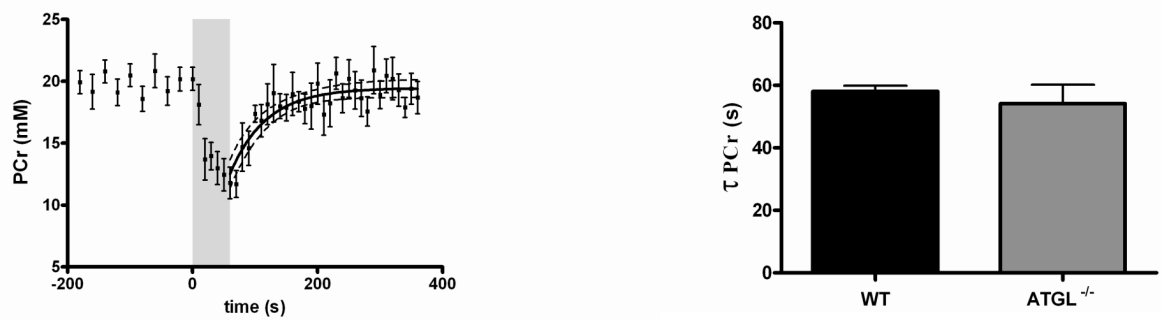

A: Example of one ${ }^{31} \mathrm{P}$ MRS time-series measured in hind-limb of $\mathrm{ATGL}^{-}$mouse during the resting-stimulationrecovery protocol. Muscle contraction was achieved by electro-stimulation of calf-muscles at a frequency of $0.33 \mathrm{~Hz}$. Resonances for inorganic phosphate ( $\mathrm{Pi})$, phosphocreatine ( $\mathrm{PCr}$ ), and adenosine triphosphate (ATP) are indicated. $\mathrm{B}$ : $\mathrm{PCr}$ concentration before, during and after the $60 \mathrm{~s}$ of electro-stimulation (grey bar). Example of the monoexponential fitting of the WT mice PCr recovery curves, with dashed lines representing $95 \%$ confidence interval. C: Average PCr recovery times for $\mathrm{ATGL}^{\%-}(n=9$; grey bar) and WT ( $n=10$; black bar) mice; Values are presented as mean \pm SEM. 


\section{Discussion:}

In this study, we demonstrated that skeletal muscle of $\mathrm{ATGL}^{-1}$ mice accumulates large amounts of IMCL and shows decreased force production and muscle relaxation, however no apparent decrease of mitochondrial oxidative capacity was detected.

\section{Physiology of the model}

Whole-body deletion of ATGL hampers TG lipolysis, resulting in decreased FFA levels and in neutral lipid accumulation in various tissues. This is reflected in $\mathrm{ATGL}^{-/}$mice body weight and is exclusively due to an increase of fat mass ${ }^{18,30}$. Glucose disposal in $\mathrm{ATGL}^{-1-}$ mice is improved, as well as insulin sensitivity, and after prolonged fasting, plasma glucose levels decrease substantially ${ }^{16,19,30}$. At rest, ATGL $^{-/}$mice have reduced glycogen stores $(\sim 30 \%)$ in skeletal muscle, which altogether point towards a reliance on carbohydrates as energy fuel when FFA are not available.

\section{Increased IMCL deposition in skeletal muscle of ATGL ${ }^{\%}$ mice}

IMCL quantification in TA of ATGL ${ }^{-/}$and WT mice by in vivo ${ }^{1} \mathrm{H}$ MRS showed that $\mathrm{ATGL}^{-1-}$ mice have a 6 fold increase in IMCL content. The TA muscle was chosen to determine the level of intra and extra-myocellular lipids as its fibres can be oriented parallel to the magnetic field, essential for a proper discrimination of the ${ }^{1} \mathrm{H}$ resonances of intra and extra-myocellular lipids ${ }^{3,4}$. Our results show that the use of small voxels $(3.1 \mu \mathrm{L})$ in localized ${ }^{1} \mathrm{H}$ MRS enable a selective determination of the IMCL pool in TA of mice, similar to what has been described for rats ${ }^{32}$.

Electron-microscopy data showed that in $\mathrm{ATGL}^{-1} \mathrm{SOL}$ muscle, the number and size of the LD is significantly higher than in fast-twitch muscle EDL. Since the TA muscle is, as the EDL, mainly composed by fast-twitch type II fibres ( $80 \%$ ), a 6 fold increase in IMCL pool is potentially an underestimation for muscles with more oxidative type of fibres. Interestingly, the electron-microscopy images also revealed that in $\mathrm{ATGL}^{-1-}$ muscles, neighbouring fibres are differently affected by lipid accumulation. This might be related to fibre phenotype, as greater lipid accumulation in $\mathrm{ATGL}^{-1-}$ mice occurs in oxidative rather than in glycolytic fibres ${ }^{47}$. Whether this fact is related to ATGL expression in specific muscle fibres, as it happens in human skeletal muscle ${ }^{21}$ remains unknown.

Regarding the possible "lipotoxic" interaction between IMCL and mitochondrial function in skeletal muscle, the nature of this lipid accumulation is somewhat of importance. It was shown that $\mathrm{ATGL}^{-1}$ skeletal muscle accumulate mainly IMCL as neutral $\mathrm{TG}^{16,19,30}$, whereas 
ceramide and overall long-chain FA contents were found unaltered or decreased in $\mathrm{ATGL}^{-/}$ SOL muscle compared to WT littermates ${ }^{30}$.

\section{In vivo mitochondrial oxidative capacity of ATGL $\%$ mice is similar to WT mice}

In order to evaluate whether skeletal muscle of $\mathrm{ATGL}^{-1-}$ mice had impaired mitochondrial function, we assessed, in vivo, mitochondrial oxidative capacity by ${ }^{31} \mathrm{P}$ MRS after maximal muscle contractions. Upon cessation of an energy demanding exercise, skeletal muscle mitochondrial oxidative phosphorylation produces ATP which is almost exclusively used to rephosphorylate $\mathrm{Cr}^{1,35}$. Hence, the $\mathrm{PCr}$ resynthesis is an in vivo measure of mitochondrial oxidative capacity and has been widely applied in human $9,26,55$ and animal studies $8,28,52$ (reviewed by ${ }^{27}$ ).

Skeletal muscle of $\mathrm{ATGL}^{-1}$ mice presented similar $\mathrm{PCr}$ recovery time ( $\mathrm{TPCr}$ ) to the WT mice, showing that $\mathrm{ATGL}^{-1}$ muscle had comparable mitochondrial oxidative capacity to their counterparts. Electron-microscopy images from SOL and EDL muscles did not show differences between $\mathrm{ATGL}^{-/}$and WT mice regarding mitochondrial localization or morphology. Others also have shown that $\mathrm{ATGL}^{-1-}$ mice on a 4-week high-fat diet, present normal skeletal muscle mitochondrial content. Maximal activities of citrate synthase and $\beta$-hydroxyacyl-CoA dehydrogenase, protein content of respiratory chain complexes and relative expression of mitochondrial biogenesis genes in the skeletal muscle were also found similar between 4-week high-fat fed $\mathrm{ATGL}^{-1}$ mice and WT mice ${ }^{18}$. When FFA are available they are promptly oxidized either ex vivo, in isolated muscle preparations ${ }^{19}$ or in vivo, when supplied by means of diet ${ }^{18}$. These studies indicate that mitochondrial $\beta$-oxidation in skeletal muscle of $\mathrm{ATGL}^{-/}$mice, is unaffected. In our study, resting or contracting muscles of $\mathrm{ATGL}^{\%}$ mice had normal ATP concentration, PCr/ATP ratio and $\mathrm{TPCr}$ compared to WT mice. Thus, remaining circulating FFA or other substrates (e.g. glucose) seemed to be sufficient to fuel mitochondrial oxidative phosphorylation and sustain the energy requirements of the muscle, at rest and during the contractions. It is possible that cardiomyopathy developed by $\mathrm{ATGL}^{-}$mice impairs blood flow and $\mathrm{O}_{2}$ supply to the working muscle and consequently the activity of mitochondrial oxidative phosphorylation. A deficiency in $\mathrm{O}_{2}$ supply would result in a decrease of intracellular $\mathrm{pH}$ and increase in $\mathrm{PCr}$ recovery time, however no differences were observed in these parameters as measured in vivo by ${ }^{31} \mathrm{P}$ MRS. Thus, within the ${ }^{31} \mathrm{P} M R S$ measurement error, the skeletal muscle mitochondrial oxidative capacity of $\mathrm{ATGL}^{-/}$mice is unaffected.

Our results indicate that factors such as the inability to generate FFA for muscle oxidation, the possible down regulation of genes involved in ATP synthesis, or the cardiac abnormalities of $\mathrm{ATGL}^{-1-}$ mice do not operate, at least in appreciable extent to cause damage in mitochondrial 
oxidative capacity. Thus, mitochondrial dysfunction in $\mathrm{ATGL}^{-1-}$ mice seems to be mainly located at the level of cardiac tissue with relative sparing of skeletal muscle.

\section{ATGL $\%$ mice have reduced force production and increased muscle relaxation time}

Despite that overall mitochondrial oxidative capacity was not compromised in $\mathrm{ATGL}^{-1}$ mice, muscle force production and relaxation, simultaneously determined, were significantly impaired.

The calf-muscle complex was electro-stimulated through the sciatic nerve with optimal current, i.e. the electric current at which the force produced was maximal. In general, in the $\mathrm{ATGL}^{-}$mice this current was significantly lower than in WT mice and yet the force produced was less than half of the force of the WT mice. This difference in force was not due to the lower optimal stimulation current, because when the same near optimal current was applied, the force generated by the $\mathrm{ATGL}^{-/}$mice remained reduced compared to WT mice. The contraction-induced $\mathrm{PCr}$ depletion in $\mathrm{WT}$ and $\mathrm{ATGL}^{-1}$ mice was comparable, which suggests that $\mathrm{ATGL}^{-1}$ mice had higher energy cost per contraction (lower contractile efficiency). In addition, the skeletal muscle of $\mathrm{ATGL}^{-/-}$mice required more time to relax than WT mice.

Our results differ from those reported previously for isolated $\mathrm{ATGL}^{-1}$ muscles, where no differences were observed in force production nor in fatigue rate of EDL and SOL under submaximal contractions ${ }^{19}$. The distinct experimental conditions, e.g. stimulation protocol/ frequency, as well as the gender of the animals used could account for the converse findings. Huijsman and co-workers used a stimulation frequency of $60 \mathrm{~Hz}$ in a muscle fatigue protocol for EDL and $\mathrm{SOL}^{19}$, whereas we used $150 \mathrm{~Hz}$ for calf muscle complex (i.e GAST and SOL) contraction. This stimulation frequency produces the highest force in GAST and $\mathrm{SOL}^{10,22,23}$. When the electro-stimulation current was lower than the optimal current, e.g. $1.25 \mathrm{~mA}$, no differences in force between $\mathrm{WT}$ and $\mathrm{ATGL}^{-/}$mice could be detected. This indicates that only when $\mathrm{ATGL}^{-/}$muscles contract at high force the difference to WT becomes apparent. The presence of defective muscle performance in $\mathrm{ATGL}^{-1}$ is supported by studies that showed that these mice are exercise intolerant with reduced maximal running velocity and endurance capacity ${ }^{19,47}$.

As contrary to our hypothesis, mitochondrial dysfunction does not explain the poor muscle performance of $\mathrm{ATGL}^{-/}$mice, other potential mechanisms must be responsible for this defect. For instance, a lower optimal stimulation current suggests differences in nerve resistance in $\mathrm{ATGL}^{-1}$ mice. This could involve the myelin sheaths that insulate the nerve and conduct electric pulses. Since myelin integrity is highly affected in numerous lipid metabolic disorders (reviewed by ${ }^{7}$ ), reduction of FFA as in $\mathrm{ATGL}^{-1-}$ mice could compromise its function. 
Another possible explanation is a mechanical contraction defect in the muscle filaments due to excessive IMCL accumulation and extremely large LD in $\mathrm{ATGL}^{-/-}$muscles. Furthermore, $\mathrm{Ca}^{2+}$ handling at the sarcoplasmic reticulum (SR) may be involved, for instance caused by lower levels of glycogen. Reductions in force production, $\mathrm{Ca}^{2+}$ release and inhibition of contractile protein are closely associated with reduced muscle glycogen ${ }^{6,37}$, due to tight association of glycogen and glycolytic enzymes to the $\mathrm{SR}^{13,31}$. A reduction in glycogen could lead to a local deficiency in ATP regeneration at the SR compromising the release of $\mathrm{Ca}^{2+}$ (reviewed by ${ }^{2}$; $14 ; 34 ; 56)$. And finally, a dysfunction in subsarcolemmal mitochondria, as observed in cardiac muscle of $\mathrm{ATGL}^{-1-}$ mice ${ }^{17}$ would also lead to compromised SR Ca ${ }^{2+}$ reuptake, increased muscle relaxation time and decreased SR $\mathrm{Ca}^{2+}$ load in successive contractions ${ }^{5 ; 45}$. But subsarcolemmal mitochondrial involvement should be limited or compensated by myofibrillar mitochondria, as within the error of the in vivo ${ }^{31} \mathrm{P}$ MRS measurement, the overall mitochondrial oxidative capacity was normal.

In conclusion, our in vivo data confirm that whole-body ATGL deletion leads to a significant $I M C L$ accumulation in muscle. Different muscles are differently affected suggesting that IMCL deposition is fibre type dependent. Despite this lipid accumulation and the inability to release fatty acids from $I M C L$, the in vivo skeletal muscle mitochondrial oxidative capacity of $\mathrm{ATGL}^{-1-}$ mice, measured here upon electro-stimulation using ${ }^{31} \mathrm{P} \mathrm{MRS}$, was comparable to that of WT mice. Electro-stimulated muscles of $\mathrm{ATGL}^{-/-}$mice exhibited reduced force production and higher relaxation times than the muscles of WT mice. As impairment of mitochondrial oxidative phosphorylation does not explain the poor $\mathrm{ATGL}^{-/}$muscle performance other mechanisms must be responsible for this defect.

\section{Acknowledgements:}

The authors would like to acknowledge Mr. W.A. Coumans for expert technical assistance, and Dr. G. Kemp for useful comments.

\section{Grants:}

This research was performed within the framework of CTMM, the Center for Translational Molecular Medicine (www.ctmm.nl), project PREDICCt (grant 01C-104), and supported by the Dutch Heart Foundation, Dutch Diabetes Research Foundation and Dutch Kidney Foundation. Additional support was obtained from EU project FAST: MRTN-CT-2006-035801. 


\section{References}

1. Arnold DL, Matthews PM and Radda GK. Metabolic recovery after exercise and the assessment of mitochondrial function in vivo in human skeletal muscle by means of 31P NMR. Magn Reson Med 1: 307-315, 1984.

2. Bendahan D, Giannesini B and Cozzone PJ. Functional investigations of exercising muscle: a noninvasive magnetic resonance spectroscopy-magnetic resonance imaging approach. Cell Mol Life Sci 61: 1001-1015, 2004.

3. Boesch C, Machann J, Vermathen P and Schick F. Role of proton MR for the study of muscle lipid metabolism. NMR Biomed 19: 968-988, 2006.

4. Boesch $\mathrm{C}$, Slotboom J, Hoppeler $\mathrm{H}$ and Kreis R. In vivo determination of intra-myocellular lipids in human muscle by means of localized 1H-MR-spectroscopy. Magn Reson Med 37: 484-493, 1997.

5. Byrd SK. Alterations in the sarcoplasmic reticulum: a possible link to exercise-induced muscle damage. Med Sci Sports Exerc 24: 531-536, 1992.

6. Chin ER and Allen DG. Effects of reduced muscle glycogen concentration on force, $\mathrm{Ca} 2+$ release and contractile protein function in intact mouse skeletal muscle. J Physiol 498 ( Pt 1): 17-29, 1997.

7. Chrast R, Saher G, Nave KA and Verheijen MH. Lipid metabolism in myelinating glial cells: lessons from human inherited disorders and mouse models. J Lipid Res 52: 419-434, 2011.

8. De Feyter HM, Lenaers E, Houten SM, Schrauwen P, Hesselink MK, Wanders RJ, Nicolay $\mathrm{K}$ and Prompers JJ. Increased intramyocellular lipid content but normal skeletal muscle mitochondrial oxidative capacity throughout the pathogenesis of type 2 diabetes. FASEB J 22: 3947-3955, 2008.

9. De Feyter HM, van den Broek NM, Praet SF, Nicolay K, van Loon LJ and Prompers JJ. Early or advanced stage type 2 diabetes is not accompanied by in vivo skeletal muscle mitochondrial dysfunction. Eur J Endocrinol 158: 643-653, 2008.

10. de Haan A. The influence of stimulation frequency on force-velocity characteristics of in situ rat medial gastrocnemius muscle. Exp Physiol 83: 77-84, 1998.

11. de Haan A, Jones DA and Sargeant AJ. Changes in velocity of shortening, power output and relaxation rate during fatigue of rat medial gastrocnemius muscle. Pflugers Arch 413: 422428, 1989.

12. Edwards RH, Hill DK and Jones DA. Metabolic changes associated with the slowing of relaxation in fatigued mouse muscle. J Physiol 251: 287-301, 1975.

13. Entman ML, Keslensky SS, Chu A and Van Winkle WB. The sarcoplasmic reticulumglycogenolytic complex in mammalian fast twitch skeletal muscle. Proposed in vitro counterpart of the contraction-activated glycogenolytic pool. J Biol Chem 255: 6245-6252, 1980.

14. Favero TG. Sarcoplasmic reticulum $\mathrm{Ca}(2+)$ release and muscle fatigue. J Appl Physiol 87: 471483, 1999.

15. Fissoune $R$, Janier $M$, Briguet $A$ and Hiba $B$. In vivo assessment of mouse hindleg intramyocellular lipids by 1H-MR spectroscopy. Acad Radiol 16: 890-896, 2009.

16. Haemmerle G, Lass A, Zimmermann R, Gorkiewicz G, Meyer C, Rozman J, Heldmaier G, Maier R, TheussI C, Eder S, Kratky D, Wagner EF, Klingenspor M, Hoefler $G$ and Zechner R. Defective 
lipolysis and altered energy metabolism in mice lacking adipose triglyceride lipase. Science 312: 734-737, 2006.

17. Haemmerle G, Moustafa T, Woelkart G, Buttner S, Schmidt A, van de Weijer T, Hesselink M, Jaeger D, Kienesberger PC, Zierler K, Schreiber R, Eichmann T, Kolb D, Kotzbeck P, Schweiger M, Kumari M, Eder S, Schoiswohl G, Wongsiriroj N, Pollak NM, Radner FP, Preiss-Landl K, Kolbe T, Rulicke T, Pieske B, Trauner M, Lass A, Zimmermann R, Hoefler G, Cinti S, Kershaw EE, Schrauwen P, Madeo F, Mayer B and Zechner R. ATGL-mediated fat catabolism regulates cardiac mitochondrial function via PPAR-alpha and PGC-1. Nat Med 17: 1076-1085, 2011.

18. Hoy AJ, Bruce CR, Turpin SM, Morris AJ, Febbraio MA and Watt MJ. Adipose triglyceride lipase-null mice are resistant to high-fat diet-induced insulin resistance despite reduced energy expenditure and ectopic lipid accumulation. Endocrinology 152: 48-58, 2011.

19. Huijsman E, van de PC, Economou C, van der PC, Lynch GS, Schoiswohl G, Haemmerle G, Zechner R and Watt MJ. Adipose triacylglycerol lipase deletion alters whole body energy metabolism and impairs exercise performance in mice. Am J Physiol Endocrinol Metab 297: E505-E513, 2009.

20. in 't Zandt HJ, de Groof AJ, Renema WK, Oerlemans FT, Klomp DW, Wieringa B and Heerschap A. Presence of (phospho)creatine in developing and adult skeletal muscle of mice without mitochondrial and cytosolic muscle creatine kinase isoforms. J Physiol 548: 847-858, 2003.

21. Jocken JW, Smit E, Goossens GH, Essers YP, van Baak MA, Mensink M, Saris WH and Blaak EE. Adipose triglyceride lipase (ATGL) expression in human skeletal muscle is type I (oxidative) fiber specific. Histochem Cell Biol 129: 535-538, 2008.

22. Jones DA and Round JM. Skeletal muscle in health an disease: a textbook of muscle physiology. Manchester: Manchester University Press, 1990.

23. Kan HE, Buse-Pot TE, Peco R, Isbrandt D, Heerschap A and de Haan A. Lower force and impaired performance during high-intensity electrical stimulation in skeletal muscle of GAMT-deficient knockout mice. Am J Physiol Cell Physiol 289: C113-C119, 2005.

24. Kan HE, Veltien A, Arnts H, Nabuurs Cl, Luijten B, de Haan A, Wieringa B and Heerschap A. Gated dynamic 31P MRS shows reduced contractile phosphocreatine breakdown in mice deficient in cytosolic creatine kinase and adenylate kinase. NMR Biomed 22: 523-531, 2009.

25. Kelley DE, Goodpaster B, Wing RR and Simoneau JA. Skeletal muscle fatty acid metabolism in association with insulin resistance, obesity, and weight loss. Am J Physiol 277: E1130-E1141, 1999.

26. Kemp GJ and Radda GK. Quantitative interpretation of bioenergetic data from 31P and $1 \mathrm{H}$ magnetic resonance spectroscopic studies of skeletal muscle: an analytical review. Magn Reson Q 10: 43-63, 1994.

27. Kemp GJ, Roberts N, Bimson WE, Bakran A, Harris PL, Gilling-Smith GL, Brennan J, Rankin $A$ and Frostick SP. Mitochondrial function and oxygen supply in normal and in chronically ischemic muscle: a combined 31P magnetic resonance spectroscopy and near infrared spectroscopy study in vivo. J Vasc Surg 34: 1103-1110, 2001.

28. Kemp GJ, Taylor DJ and Radda GK. Control of phosphocreatine resynthesis during recovery from exercise in human skeletal muscle. NMR Biomed 6: 66-72, 1993.

29. Kershaw EE, Hamm JK, Verhagen LA, Peroni O, Katic M and Flier JS. Adipose triglyceride lipase: function, regulation by insulin, and comparison with adiponutrin. Diabetes 55: 148157, 2006.

30. Kienesberger PC, Lee D, Pulinilkunnil T, Brenner DS, Cai L, Magnes C, Koefeler HC, Streith IE, Rechberger GN, Haemmerle G, Flier JS, Zechner R, Kim YB and Kershaw EE. Adipose 
triglyceride lipase deficiency causes tissue-specific changes in insulin signaling. J Biol Chem 284: 30218-30229, 2009.

31. Korge P and Campbell KB. The importance of ATPase microenvironment in muscle fatigue: a hypothesis. Int J Sports Med 16: 172-179, 1995.

32. Kuhlmann J, Neumann-Haefelin C, Belz U, Kalisch J, Juretschke HP, Stein M, Kleinschmidt E, Kramer W and Herling AW. Intramyocellular lipid and insulin resistance: a longitudinal in vivo 1H-spectroscopic study in Zucker diabetic fatty rats. Diabetes 52: 138-144, 2003.

33. Lake AC, Sun Y, Li JL, Kim JE, Johnson JW, Li D, Revett T, Shih HH, Liu W, Paulsen JE and Gimeno RE. Expression, regulation, and triglyceride hydrolase activity of Adiponutrin family members. J Lipid Res 46: 2477-2487, 2005.

34. Lees SJ and Williams JH. Skeletal muscle sarcoplasmic reticulum glycogen status influences Ca2+ uptake supported by endogenously synthesized ATP. Am J Physiol Cell Physiol 286: C97104, 2004.

35. Meyer RA. A linear model of muscle respiration explains monoexponential phosphocreatine changes. Am J Physiol 254: C548-C553, 1988.

36. Naressi A, Couturier C, Devos JM, Janssen M, Mangeat C, de BR and Graveron-Demilly D. Java-based graphical user interface for the MRUI quantitation package. MAGMA 12: 141152, 2001.

37. Nielsen J, Schroder HD, Rix CG and Ortenblad N. Distinct effects of subcellular glycogen localization on tetanic relaxation time and endurance in mechanically skinned rat skeletal muscle fibres. J Physiol 587: 3679-3690, 2009.

38. Ong KT, Mashek MT, Bu SY, Greenberg AS and Mashek DG. Adipose triglyceride lipase is a major hepatic lipase that regulates triacylglycerol turnover and fatty acid signaling and partitioning. Hepatology 53: 116-126, 2011.

39. Parish R and Petersen KF. Mitochondrial dysfunction and type 2 diabetes. Curr Diab Rep 5: 177-183, 2005.

40. Petersen KF, Dufour S, Befroy D, Garcia R and Shulman GI. Impaired mitochondrial activity in the insulin-resistant offspring of patients with type 2 diabetes. N Engl J Med 350: 664-671, 2004.

41. Peyot ML, Guay C, Latour MG, Lamontagne J, Lussier R, Pineda M, Ruderman NB, Haemmerle G, Zechner R, Joly E, Madiraju SR, Poitout V and Prentki M. Adipose triglyceride lipase is implicated in fuel- and non-fuel-stimulated insulin secretion. J Biol Chem 284: 16848-16859, 2009.

42. Pinent M, Hackl H, Burkard TR, Prokesch A, Papak C, Scheideler M, Hammerle G, Zechner R, Trajanoski $Z$ and Strauss JG. Differential transcriptional modulation of biological processes in adipocyte triglyceride lipase and hormone-sensitive lipase-deficient mice. Genomics 92: 26-32, 2008.

43. Popesco P, Rajtová V and Horák J. A Colour atlas of the anatomy of small laboratory animals. 2003.

44. Roden M. Muscle triglycerides and mitochondrial function: possible mechanisms for the development of type 2 diabetes. Int J Obes (Lond) 29 Suppl 2: S111-S115, 2005.

45. Rossi AE, Boncompagni S, Wei L, Protasi F and Dirksen RT. Differential Impact of Mitochondrial Positioning on Mitochondrial Ca2+ Uptake and Ca2+ Spark Suppression in Skeletal Muscle. Am J Physiol Cell Physiol 2011.

46. Samuel VT, Petersen KF and Shulman GI. Lipid-induced insulin resistance: unravelling the mechanism. Lancet 375: 2267-2277, 2010. 
47. Schoiswohl G, Schweiger M, Schreiber R, Gorkiewicz G, Preiss-Landl K, Taschler U, Zierler KA, Radner FP, Eichmann TO, Kienesberger PC, Eder S, Lass A, Haemmerle G, Alsted TJ, Kiens B, Hoefler G, Zechner R and Zimmermann R. Adipose triglyceride lipase plays a key role in the supply of the working muscle with fatty acids. J Lipid Res 51: 490-499, 2010.

48. Schrauwen-Hinderling VB, Roden M, Kooi ME, Hesselink MK and Schrauwen P. Muscular mitochondrial dysfunction and type 2 diabetes mellitus. Curr Opin Clin Nutr Metab Care 10: 698-703, 2007.

49. Shortreed KE, Krause MP, Huang JH, Dhanani D, Moradi J, Ceddia RB and Hawke TJ. Musclespecific adaptations, impaired oxidative capacity and maintenance of contractile function characterize diet-induced obese mouse skeletal muscle. PLoS One 4: e7293, 2009.

50. Taylor DJ, Bore PJ, Styles P, Gadian DG and Radda GK. Bioenergetics of intact human muscle. A 31P nuclear magnetic resonance study. Mol Biol Med 1: 77-94, 1983.

51. Turpin SM, Hoy AJ, Brown RD, Rudaz CG, Honeyman J, Matzaris M and Watt MJ. Adipose triacylglycerol lipase is a major regulator of hepatic lipid metabolism but not insulin sensitivity in mice. Diabetologia 54: 146-156, 2011.

52. van den Broek NM, Ciapaite J, De Feyter HM, Houten SM, Wanders RJ, Jeneson JA, Nicolay K and Prompers JJ. Increased mitochondrial content rescues in vivo muscle oxidative capacity in long-term high-fat-diet-fed rats. FASEB J 24: 1354-1364, 2010.

53. Vanhamme L, van den BA and Van HS. Improved method for accurate and efficient quantification of MRS data with use of prior knowledge. J Magn Reson 129: 35-43, 1997.

54. Villena JA, Roy S, Sarkadi-Nagy E, Kim KH and Sul HS. Desnutrin, an adipocyte gene encoding a novel patatin domain-containing protein, is induced by fasting and glucocorticoids: ectopic expression of desnutrin increases triglyceride hydrolysis. J Biol Chem 279: 47066-47075, 2004.

55. Walter $\mathrm{G}$, Vandenborne $\mathrm{K}$, Elliott $\mathrm{M}$ and Leigh JS. In vivo ATP synthesis rates in single human muscles during high intensity exercise. J Physiol 519 Pt 3: 901-910, 1999.

56. Westerblad H, Allen DG, Bruton JD, Andrade FH and Lannergren J. Mechanisms underlying the reduction of isometric force in skeletal muscle fatigue. Acta Physiol Scand 162: 253-260, 1998.

57. Wynants J and Van Belle H. Single-run high-performance liquid chromatography of nucleotides, nucleosides, and major purine bases and its application to different tissue extracts. Anal Biochem 144: 258-266, 1985.

58. Zimmermann R, Strauss JG, Haemmerle G, Schoiswohl G, Birner-Gruenberger R, Riederer M, Lass A, Neuberger G, Eisenhaber F, Hermetter A and Zechner R. Fat mobilization in adipose tissue is promoted by adipose triglyceride lipase. Science 306: 1383-1386, 2004. 


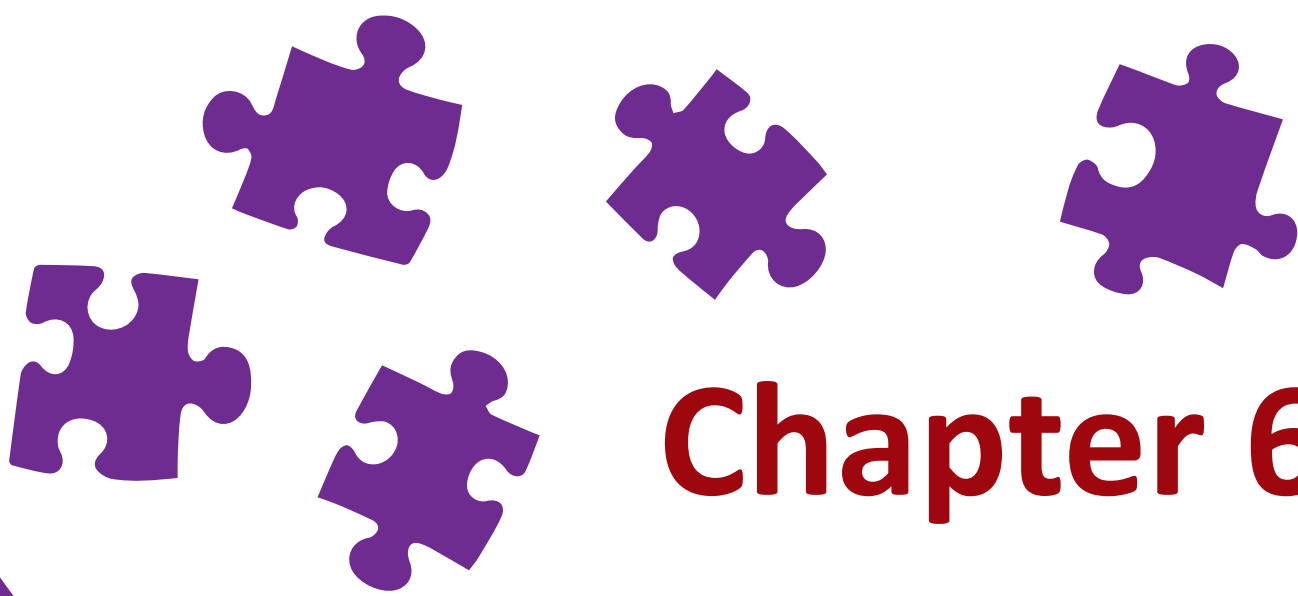

Chapter 6

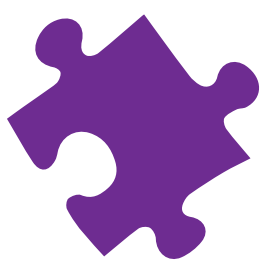

\section{Effects of bezafibrate treatment in a patient} and a carrier with mutations in the PNPLA2 gene, causing Neutral Lipid Storage Disease with Myopathy (NLSDM)

Tineke van de Weijer ${ }^{1}$, MD, Bas Havekes ${ }^{4}, \mathrm{MD}, \mathrm{PhD}$, Lena Bilet ${ }^{1}$, MSc, Joris Hoeks ${ }^{1}$, PhD, Lauren Sparks ${ }^{1}, \mathrm{PhD}$, Madeleen Bosma ${ }^{1}, \mathrm{MSc}$, Sabina Paglialunga ${ }^{1}$, PhD, Johanna Jorgensen ${ }^{1}$, MSc, Mirian C.H. Janssen ${ }^{6}$, MD, Gert Schaart ${ }^{2}$, PhD, Hans Sauerwein ${ }^{4}$, MD PhD, Joep L. Smeets ${ }^{5}$, MD, PhD, Joachim Wildberger ${ }^{3}$, MD, PhD, Rudolf Zechner ${ }^{7}, \mathrm{PhD}$, Vera B. Schrauwen-Hinderling ${ }^{3}$, PhD, Matthijs K.C. Hesselink ${ }^{2}$, PhD, and Patrick Schrauwen ${ }^{1}$, PhD

${ }^{1}$ Departments of Human Biology, ${ }^{2}$ Human Movement Sciences, ${ }^{3}$ Radiology and ${ }^{4}$ Internal Medicine, Division of Endocrinology, NUTRIM School for Nutrition, Toxicology and Metabolism, Maastricht University Medical Center,

Maastricht, The Netherlands, Department of ${ }^{5}$ Cardiology and ${ }^{6}$ General Internal Medicine, Radboud University Nijmegen Medical Centre, Nijmegen, the Netherlands, and 'Institute of Molecular Biosciences, University of Graz,

Graz, Austria. 


\section{Introduction}

Neutral lipid storage disease with myopathy (NLSDM) is a rare but severe, genetic disorder characterized by excessive lipid accumulation in tissues including skin, bone marrow, heart, liver and muscles. Clinically, NLSDM patients present with severe dilated cardiomyopathy, skeletal muscle myopathy and insulin resistance ${ }^{1}$. NLSDM is caused by a defect in the PNPLA2 gene encoding the enzyme adipose triglyceride lipase (ATGL) ${ }^{2}$, which catalyzes the breakdown of triglycerides in multiple tissues and is the rate-limiting step of lipolysis. While heterozygous carriers and homozygous patients both present with similar clinical symptoms, the severity of these symptoms in homozygous patients is more dramatic, leading to premature death due to dilated cardiomyopathy in some patients ${ }^{1,2}$. To date, the only available treatment is strict dietary guidelines and is focused on treating the comorbidities rather than targeting the primary defect.

To investigate cardiac lipotoxicity in NLSDM, ATGL-deficient mice have been investigated. Just like NLSDM patients, ATGL-deficient mice are also characterized by excessive lipid storage in skeletal muscle, liver and heart, and develop cardiomyopathy at a young age, resulting in premature death ${ }^{3}$. Interestingly, we recently reported that lack of ATGL resulted in a diminished cardiac PPAR activity and mitochondrial function, suggesting that lipolysisderived fatty acids or fatty acid byproducts serve as transcriptional activators of PPARs ${ }^{3}$. Subsequently, pharmacological treatment of ATGL-deficient mice with PPAR agonists completely reversed the mitochondrial defects, restored cardiac function and prevented premature death. These promising findings inspired us to investigate if PPAR agonist treatment in patients and carriers of a PNPLA2 gene defect could also have beneficial effects. 


\section{Materials and Methods}

\section{Patients}

Although NSLDM is a very rare disease, we had the opportunity to study two sisters with PNPLA2 gene mutations. Patient 1 is a 37 -year-old woman with a BMI of $21.4 \mathrm{~kg} / \mathrm{m}^{2}$. During childhood, patient 1 developed hearing loss and progressive proximal muscle weakness. Histological analysis revealed increased lipid accumulation in skeletal muscle and Jordan bodies in white blood cells. At the age of 34 , she developed a diffuse hypokinesia of the left and right ventricle of the heart with a decreased ejection fraction (EF). Because of a nonsustained ventricular tachycardia, she received an implantable cardioverter defibrillator (ICD). The diagnosis of NLSDM in this patient was made after a DNA sequence analysis, showing 2 different mutations in both PNPLA2 alleles, causing a compound heterozygous mutation: a deletion causing a frame-shift at amino acid position 270 (FS270) and a missense mutation $584 \mathrm{C} \rightarrow \mathrm{T}$, that changes proline to leucine at amino acid 195 as previously described $^{1}$.

Patient 2 is the 39 -year-old sister of patient 1 , with a BMI of $28.2 \mathrm{~kg} / \mathrm{m}^{2}$. She is a heterozygous carrier of the FS270 frameshift mutation, and presents with complaints, albeit milder than her sister. Clinically, the patient is characterized by mild myopathy, reduced exercise tolerance, a history of chronic otitis media and one episode of meningitis. A muscle biopsy and the presence of Jordan bodies confirmed neutral lipid storage.

\section{Intervention}

Both patients were initially treated for 12 weeks, with a follow-up at 28 weeks, with bezafibrate (Bezalip, Actavis $^{\circledR}, 400 \mathrm{mg}$ daily). Amongst the fibrate-like PPAR-alpha agonists, bezafibrate is the only fibrate that is shown to also have PPAR-gamma and -delta activity. Patients were under strict medical control and measurements of creatinine, creatine kinase (CK) and liver enzymes were performed at 1, 2, 3, 6, 9, 12 and 28 weeks of treatment. Bezafibrate was generally well-tolerated and neither patient experienced changes in musculoskeletal or cardiac symptoms. No significant changes in basal laboratory parameters were found throughout the treatment period (also see Table 1).

To evaluate the effect of bezafibrate treatment, measurements of cardiac function, muscle metabolism and insulin sensitivity were performed before and after 12 weeks of bezafibrate treatment. To evaluate the long-term effects, the clinically most relevant parameters were also determined after 28 weeks of treatment. Although health status improved in both patients, not all measurement could be repeated at week 28 . These measurements were 
also performed in $4 \mathrm{BMI}$ and age matched female control subjects (age: $33 \pm 5$ years; $\mathrm{BMI}$ $27.8 \pm 2.2 \mathrm{~kg} / \mathrm{m}^{2}$ ), who served as a patient specific reference group and did not receive any treatment.

\section{Insulin sensitivity}

In all patients a 3-h hyperinsulinemic-euglycemic clamp was performed according to DeFronzo ${ }^{4}$, and metabolic clearance rate of glucose (MCR) was calculated ${ }^{5}$, before and after 12 weeks of treatment. Briefly, patients came to the laboratory at 8 AM in the fasted state, and bed-rested for 30 minutes. A percutaneous needle muscle biopsy of the vastus lateralis muscle was taken under local lidocaine anaesthesia ${ }^{6}$ (which was also repeated after 28 weeks of treatment). Then, two teflon cannulas were inserted and fasted plasma samples were obtained. After 30 minutes a primed constant infusion of regular insulin (Actrapid, NovoNordisk, Denmark) was started $\left(40 \mathrm{mU} / \mathrm{m}^{2} / \mathrm{min}\right)$ and plasma glucose levels were clamped at fasting plasma glucose levels ( $5-6 \mathrm{mmol} / \mathrm{l})$ by variable co-infusion of $20 \%$ glucose. Glucose infusion rates are used as a measure for insulin sensitivity after correction for weight and fasting plasma glucose, giving metabolic clearance rates of glucose. In a previous study it was shown that for repeated measures GIR showed a high correlation $(r=0.70)$ and a low coefficient of variation $(14.7 \%)^{7}$.

\section{Indirect Calorimetry}

Indirect calorimetry (ventilated hood) was performed in the fasted (before, after 12 and 28 weeks) and insulin-stimulated state (before and after 12 weeks of treatment). Oxygen consumption and carbon dioxide production was measured with an automated respiratory gas analyzer using a ventilated hood system (Omnical; IDEE, Maastricht, The Netherlands). The gas analyzer system was calibrated by an alcohol combustion test before every experiment. During the experiment oxygen and carbon dioxide levels were measured every 5 seconds over a period of 30 minutes. The average values of oxygen and carbon dioxide over this period were used. From the oxygen consumption and carbon dioxide production levels, whole body glucose oxidation and fat oxidation rates could be calculated using stoichiometric equations according to $\mathrm{Frayn}^{8}$ with the assumption that protein oxidation was negligible. Previous studies have shown, that results for these tests are highly reproducible, with no significant differences in fasting resting energy expenditure over a 2 month period (in case the subject is weight stable) ${ }^{9}$. 


\section{Skeletal muscle lipid accumulation}

Skeletal muscle biopsies were analysed for lipid content and mitochondrial function (both before and after 12 and 28 weeks of treatment). Skeletal muscle lipid content was determined by Oil Red $\mathrm{O}$ staining in cryosections of the vastus lateralis muscle ${ }^{10}$. For every time period 15 images were obtained for histological examination. The average area of ORO staining was calculated by a semi-automated program and expressed as a percentage of the whole fiber area. As the intensity of the staining may vary, all images were processed at the same time, to reduce variation in the staining procedure.

\section{Mitochondrial function}

Ex vivo mitochondrial function was quantified by measuring oxygen consumption polarographically in quadruplicates using a two-chamber Oxygraph (OROBOROS Instruments) in permeabilized fibers obtained from the vastus lateralis biopsy as described elsewhere ${ }^{11}$. Reproducibility of this measurement has been proven to be very high, with a coefficient of variation in Bakers yeast at PH 7.1, RT of 6.7\% (O2k-protocol; Mitochondrial Physiology Network 15.09: 1-3 (2012)).

Ex vivo fat and glucose oxidation was measured in homogenized muscle by $\left[1-{ }^{14} \mathrm{C}\right]$-palmitate and $\mathrm{D}\left[\mathrm{U}-{ }^{14} \mathrm{C}\right]$ glucose conversion to ${ }^{14} \mathrm{CO}_{2}$ respectively, and normalized to protein content as previously described ${ }^{12}$. As this method uses tissue homogenates, single measurements are done. Nonetheless, repeated measures have shown that reproducibility of these measurements are quite high (with a coefficient of variation of $4.5 \%$ ).

\section{Muscle strength tests}

Muscle strength testing was performed at baseline, 6, 12 and 28 weeks of treatment. At 28 weeks of treatment, only patient 2 was willing to repeat this measurement. Lower body muscle strength was tested by one-legged weight pulling until exhaustion. Upper body strength was measured with a handgrip dynamometer as previously described ${ }^{13}$. Hand dynamometer measurements were done for both the left and the right arm and measurements were repeated 3 times for both arms.

\section{Cardiac function}

At all time-points, cardiac function was estimated using ultrasound. M-mode, twodimensional and Doppler echocardiography were performed, using a Vivid 7 ultrasound system (GE Healthcare, Milwaukee, WI, USA) with 3.5 MHz cardiac transducer. Results were 
interpreted according to the criteria of the American Societies of Echocardiography, in a blinded fashion by an independent cardiologist.

\section{Cardiac and Hepatic lipid accumulation}

In patient 2, we performed additional measurements by magnetic resonance spectroscopy (MRS), before and after 12 and 28 weeks of treatment. These measurements were not possible in patient 1 due to the presence of an ICD. Cardiac lipid accumulation was determined using ${ }^{1} \mathrm{H}-\mathrm{MRS}$ on a $1.5 \mathrm{~T}$ whole-body scanner (Intera; Philips Medical Systems, Best, the Netherlands) as previously described ${ }^{14}$. A reference measurement was done with 32 averages to calculated cardiac water content. Next a watersuppressed spectrum was aquired with 64 averages in order to assess the $\mathrm{CH}_{2}$ resonance of the fat. This measurement was repeated once. Cardiac fat content is reported as percentage of the $\mathrm{CH}_{2}$ resonance of the lipids relative to the water resonance. Previous measurements for reproducibility showed a coefficient of variation of $10 \%$. At the end of the cardiac MRS examination, fat-selective MRI was performed (by in-phase/opposed-phase imaging with a dual echo sequence with echo times $=2.3 / 4.6 \mathrm{~ms}$, repetition time $=74.8 \mathrm{~ms}$, and echo times $=4.6 / 9.2 \mathrm{~ms}$ for $\mathrm{T} 2 *$ correction) in order to assess hepatic fat content. The fat/water ratio was calculated (corrected for T1 and $\mathrm{T} 2 *$ relaxation) and given as percentage. From this, absolute liver fat percentage (in weight/ weight percentage) can be estimated. Coefficient of variation for this measurement at $3 \mathrm{~T}$ was $11 \%$. 


\section{Results}

As expected, fibrate treatment resulted in a decrease in circulating triglyceride (TG) levels (-20\% in patient $1 ;-65 \%$ in patient 2 , Table 1 ). Meanwhile, no changes in other circulating plasma parameters were observed. In both patients, metabolic clearance rate, a measure of whole-body insulin sensitivity measured using a hyperinsulinemic eugclycemic clamp ${ }^{15}$, was lower compared to controls but slightly improved after 12 weeks of bezafibrate treatment (Table 1). Furthermore, both patients showed a marked increase in metabolic flexibility (= change in Respiratory Quotient (RQ) from fasted- to insulin-stimulated condition) after bezafibrate treatment (delta-RQ from 0.03 to 0.16 in patient 1 , and 0.09 to 0.17 in patient 2 vs controls delta $R Q=0.12$ ).

Whole-body fat oxidation, measured in the fasted state, slightly increased after 12 and 28 weeks of treatment in both patients, whereas fasting glucose oxidation rates declined over this intervention period, normalizing to values found in controls (figure $1 \mathrm{~A}$ ). Ex vivo FA oxidative capacity was determined in muscle biopsies. Unfortunately, biopsy yield in patient 2 was too small to perform this assay at week 0 . In both patients ex vivo fatty acid oxidation markedly improved and normalized towards control values (figure 1B), with no marked changes in ex vivo glucose oxidation (data not shown). Furthermore, ex vivo mitochondrial function (determined in permeabilized muscle fibers) upon addition of octanoyl-carnitine showed similar improvements as the palmitate oxidation assay (data not shown). These

Figure 1: Changes in substrate oxidation and mitochondrial function in NLSDM patients upon Bezafibrate treatment

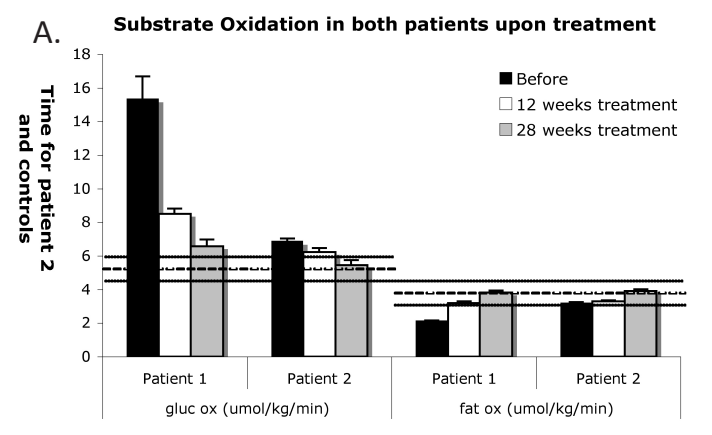

B.

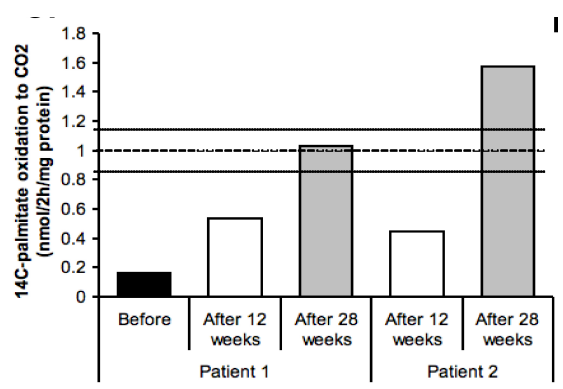

In A. whole body fat and glucose oxidation are measured after an overnight fast using indirect calorimetry, and in B. ex vivo palmitate oxidation to $\mathrm{CO}_{2}$ was measured in muscle homogenates. Black bars represent before, open bars represent after 12 weeks and gray bars represent after 28 weeks of bezafibrate treatment. In all graphs, reference values (as measured in our control subjects) are indicated by the dotted (average) and solid (SD) lines. 
data indicate that - as in ATGL deficient mice - fatty acid oxidation in these patients improved upon fibrate treatment.

Table 1: Patient Characteristics and Outcome Parameters

\begin{tabular}{|c|c|c|c|c|c|c|c|c|c|c|}
\hline & & \multicolumn{3}{|c|}{ Controls } & \multicolumn{3}{|c|}{ Patient 1} & \multicolumn{3}{|c|}{ Patient 2} \\
\hline & & \multicolumn{3}{|c|}{$(n=4)$} & before & $\begin{array}{c}12 \\
\text { weeks }\end{array}$ & $\begin{array}{c}28 \\
\text { weeks }\end{array}$ & before & $\begin{array}{c}12 \\
\text { weeks }\end{array}$ & $\begin{array}{c}28 \\
\text { weeks }\end{array}$ \\
\hline \multirow[t]{14}{*}{ Basal } & age & 33 & \pm & 5 & 37 & - & - & 39 & - & - \\
\hline & body weight (kg) & 80.8 & \pm & 8.3 & 70.4 & 71.2 & 70.4 & 79.4 & 80.2 & 80.4 \\
\hline & $\mathrm{BMI}(\mathrm{kg} / \mathrm{m} 2)$ & 27.8 & \pm & 2.2 & 21.4 & 21.6 & 21.5 & 27.7 & 27.9 & 27.9 \\
\hline & body fat \% & 34.2 & \pm & 1.4 & 32.3 & 31.6 & - & 38.3 & 39.3 & - \\
\hline & Total Cholesterol (mmol/l) & 5.4 & \pm & 0.4 & 4.3 & 3.4 & 3.7 & 4.8 & 5.1 & 4.3 \\
\hline & $\mathrm{HDL}(\mathrm{mmol} / \mathrm{l})$ & 1.8 & \pm & 0.2 & 1.2 & 1.1 & 1.3 & 1.1 & 1.4 & 1.3 \\
\hline & LDL (mmol/l) & 3.1 & \pm & 0.4 & 2.7 & 1.8 & 2.2 & 2.9 & 3.1 & 2.7 \\
\hline & triglycerides (mmol/l) & 1.22 & \pm & 0.37 & 0.96 & 0.59 & 0.66 & 1.91 & 0.92 & 0.65 \\
\hline & Free fatty acids (mmol/L) & 0.47 & \pm & 1.30 & 0.15 & 0.20 & 0.30 & 0.64 & 0.08 & 0.40 \\
\hline & Creatinine (mmol/l) & 73 & \pm & 4.5 & 61 & 56 & 68 & 60 & 72 & 82 \\
\hline & CK (U/I) & 64 & \pm & 8.8 & 307 & 290 & 266 & 61 & 88 & 81 \\
\hline & ALAT (U/I) & 18 & \pm & 3.1 & 26 & 26 & 29 & 15 & 19 & 15 \\
\hline & ASAT (U/I) & 45 & \pm & 8.8 & 32 & 34 & 32 & 12 & 21 & 16 \\
\hline & gamma-GT & 21 & \pm & 1.4 & 26 & 20 & 17 & 19 & 24 & 16 \\
\hline \multirow[t]{6}{*}{ Clamp } & $\begin{array}{l}\text { MCR-value (ml/(kg.min)) } \\
\text { glucose (mmol/l) }\end{array}$ & 8.5 & \pm & 1.0 & 6.2 & 6.6 & - & 3.4 & 3.9 & - \\
\hline & basal & 4.5 & \pm & 0.5 & 4.9 & 5 & - & 5.4 & 5.3 & - \\
\hline & during clamp & 5.5 & \pm & 0.5 & 6.4 & 5.3 & - & 6.6 & 5.2 & - \\
\hline & insulin (mU/l) & & & & & & & & & \\
\hline & basal & 4.8 & \pm & 0.9 & 8.6 & 7.9 & - & 8.5 & 8.9 & - \\
\hline & during clamp & & - & & 99.2 & 91.7 & - & 66.2 & 66.8 & - \\
\hline \multirow[t]{5}{*}{ US } & Heart rate (beats/min) & 60 & \pm & 5.0 & 76 & 72 & 76 & 74 & 77 & 79 \\
\hline & $\% F S(\%)$ & 30 & \pm & 4.13 & 16 & 10 & - & 42 & 40 & 39 \\
\hline & LVEF MOD (A4C) (\%) & 57 & \pm & 6.03 & 50 & 54 & 53 & 72 & 69 & 63 \\
\hline & LV mass (ASE) (g) & 114 & \pm & 8.0 & 226 & 247 & 278 & 108 & 131 & 142 \\
\hline & Rel. WT & 0.84 & \pm & 0.01 & 1.18 & 1.17 & 1.26 & 0.95 & 0.88 & 0.98 \\
\hline
\end{tabular}

Results are presented for controls and both patients before and after 12 and 28 weeks of treatment with Bezafibrate. Plasma values are determined after an overnight fast and during a hyperinsulinemic euglycemic clamp. Ultrasound results are presented for 2-Dimensional (2D) imaging. Abbreviations; MCR: metabolic clearance rate of glucose, FS $=$ fractional shortening, LVEF = left ventricular ejection fraction, LV mass = left ventricular mass calculated according to the ASE convention and Rel WT = relative wall thickness. 


\section{Figure 2: Changes in ectopic fat accumulation}

A.
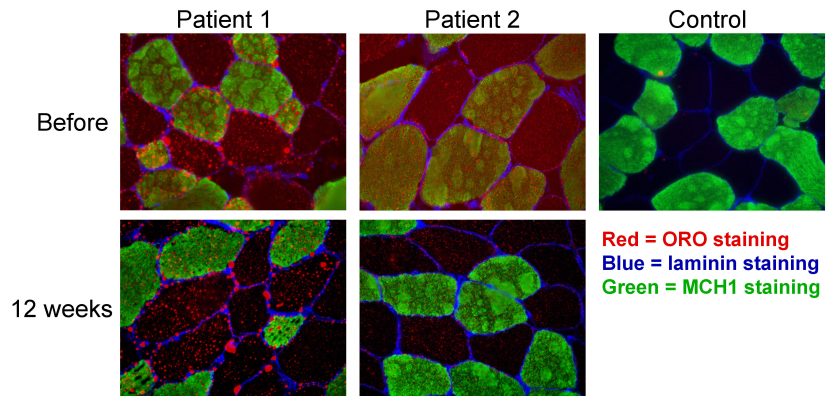

Red $=$ ORO staining

Blue = laminin staining

Green = MCH1 staining

28 weeks
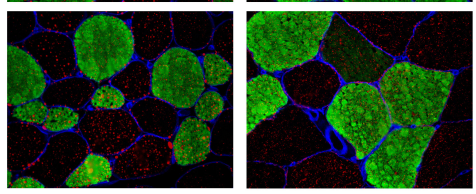

B.

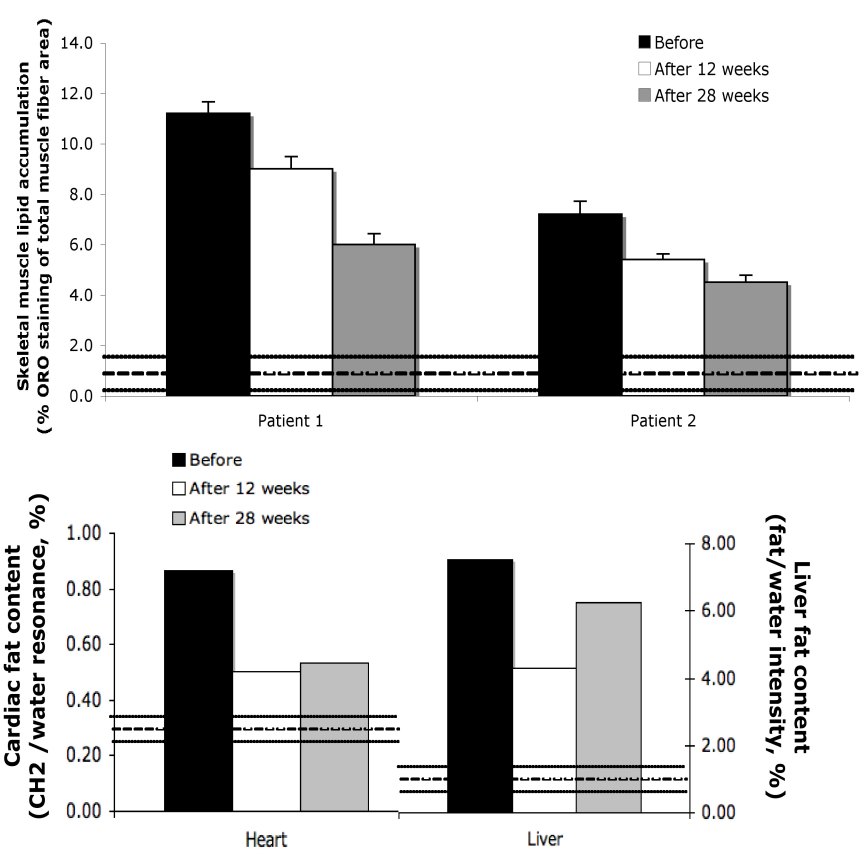

In A. Representative images of skeletal muscle lipid content, measured with ORO staining in muscle biopsies of the vastus lateralis muscle and in B. a quantitative bar graph representing the average lipid accumulation of these images C. MRS analysis in patient 2 of cardiac lipid content, given as the ratio of the $\mathrm{CH}_{2} /$ water resonance (\%) and hepatic lipid content (right axis) given as fat/water ratios (\%, after T1 and T2* correction) as determined by fatselective MRI. Black bars represent before, open bars represent after 12 weeks and gray bars represent after 28 weeks of bezafibrate treatment. In all graphs, reference values (as measured in our control subjects) are indicated by the dotted (average) and solid (SD) lines. 
Most importantly, skeletal muscle lipid content, measured in muscle biopsies using oil red $\mathrm{O}$ staining, strongly decreased in both patients upon treatment, although remained elevated compared to controls (figure $2 \mathrm{~A}+\mathrm{B}$ ). Lipid accumulation in cardiac muscle and liver in patient 2, measured with ${ }^{1} \mathrm{H}-\mathrm{MRS}^{16}$, also seemed to decrease over the 28 week intervention period (figure $2 \mathrm{C}$ ). Due to the presence of an ICD, these MRS measurements could not be performed in patient 1 .

Despite a marked decrease in tissue lipid accumulation and an improvement in oxidative metabolism, no major changes were (yet) found in clinical parameters. Handgrip strength was low in NLSDM patients and did not change upon bezafibrate treatment in either subject (data not presented). However, both patients did show an improvement in a leg extension test, a measure of leg muscle strength (figure 3). Albeit this did seem to consistently improve, the values for both patients remained low compared to age, BMI and gender matched controls (dotted lines figure 3).

Figure 3: Changes in muscle strength upon treatment

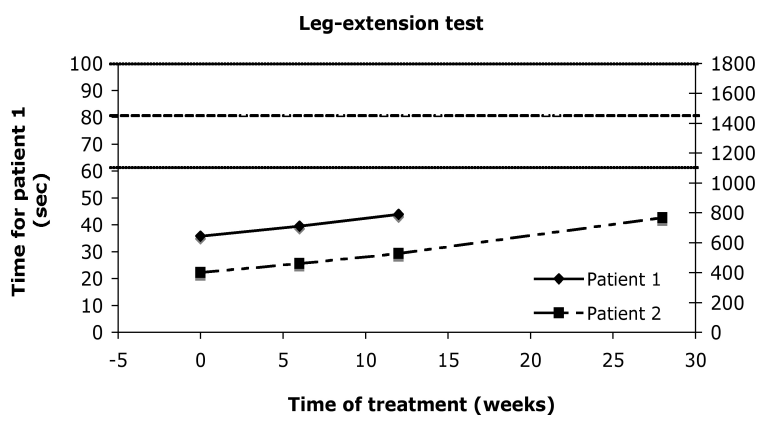

Muscle strength was measured by one-legged weight pulling and is expressed as time until exhaustion. Reference values (as measured in our control subjects) are indicated by the dotted (average) and solid (SD) lines.

Cardiac function at baseline was impaired in patient 1 and normal in patient 2 (table 1). Patient 1, who is known to have frequent arrhythmias prior to treatment, only had one episode with a non-sustained ventricular tachycardia during the intervention period, as registered by the ICD. Still, neither the ultrasound nor the ECG could detect marked changes in cardiac function in both patients after 28 weeks of treatment (table 1). 


\section{Discussion}

Consistent with the findings in mice lacking $A T G L^{17}$, bezafibrate treatment resulted in a marked lowering of cardiac and muscle fat content and an increase in fat oxidative capacity. In healthy subjects, fibrates were shown not to affect ectopic lipid content ${ }^{18,19}$, suggesting that fibrates may reduce the extreme lipid accumulation that characterizes NLSDM by upregulating and normalizing oxidative metabolism. Haemmerle et al. ${ }^{17}$ recently showed that the lack of ATGL prevents liberation of fatty acids (or FA intermediates) for PPAR signaling from the lipid droplet hence resulting in an impaired fat oxidative capacity. As a consequence, oxidation of circulatory fatty acids taken up in muscle, liver and heart is compromised and these FA will be more readily directed to storage. By providing synthetic PPAR-agonists to ATGL deficient mice fat oxidative capacity could be increased, ectopic fat storage was blunted and cardiac dysfunction completely restored. The results presented here indicate that in humans with compromised ATGL activity, PPAR treatment results in similar improvements in fat oxidation and tissue lipid storage as in ATGL deficient mice. However, unlike ATGL deficient mice, the improvements in lipid accumulation and oxidative metabolism observed in our patients did not (yet) result in pronounced clinical changes. Leg muscle strength did improve but was not paralleled by a decrease/normalization of CK levels, nor a change in handgrip strength. Furthermore cardiac function seemed unaltered. Whether the lack of clinical changes are due to the relative short duration of the treatment, or the fact that treatment could only be started in patients that are in a later stage of the disease - note that ATGL deficient mice were treated from early age onwards - can not be deduced from the present study and needs further investigation.

In conclusion, our results suggest that - similar as mice lacking ATGL - NLSDM patients may also benefit from bezafibrate treatment with respect to lipid accumulation and fat oxidative capacity. However, 28 weeks of bezafibrate treatment did not result in clinical changes in these patients. Longer studies are needed to investigate if these improvements can also translate into clinical improvements in these patients. 


\section{Acknowledgements}

The authors thank Jasper Most and Ellen Blaak for their help with including and measuring the controls of this study.

\section{Sources of funding}

P.S. is supported by a $\mathrm{VICl}$ Research Grant for innovative research from the Netherlands Organization for Scientific Research (Grant 918.96.618). T.v.d.W. was supported by the Center for Translational Molecular Medicine (CTMM) project PREDICCt (Grant 01C-104) and the Netherlands Heart Foundation, Dutch Diabetes Research Foundation, and Dutch Kidney Foundation. V.S. is supported by a VENI research grant for innovative research from the Netherlands Organization for Scientific Research (Grant 916.11.136). R.Z. was supported by "SFB LIPOTOX" F30, and the "Wittgenstein Award 2007" Z136. 


\section{References}

1. Fischer J, Lefevre C, Morava E, Mussini JM, Laforet P, Negre-Salvayre A, Lathrop M, Salvayre $R$. The gene encoding adipose triglyceride lipase (pnpla2) is mutated in neutral lipid storage disease with myopathy. Nat. Genet. 2007;39:28-30

2. Schweiger M, Lass A, Zimmermann R, Eichmann TO, Zechner R. Neutral lipid storage disease: Genetic disorders caused by mutations in adipose triglyceride lipase/pnpla2 or cgi-58/ abhd5. Am. J. Physiol. Endocrinol. Metab. 2009;297:E289-296

3. Haemmerle G, Lass A, Zimmermann R, Gorkiewicz G, Meyer C, Rozman J, Heldmaier G, Maier $R$, TheussI C, Eder S, Kratky D, Wagner EF, Klingenspor M, Hoefler G, Zechner R. Defective lipolysis and altered energy metabolism in mice lacking adipose triglyceride lipase. Science. 2006;312:734-737

4. Defronzo RA, Tobin JD, Andres R. Glucose clamp technique: A method for quantifying insulin secretion and resistance. The American journal of physiology. 1979;237:E214-223

5. Doberne L, Greenfield MS, Rosenthal M, Widstrom A, Reaven G. Effect of variations in basal plasma glucose concentration on glucose utilization $(\mathrm{m})$ and metabolic clearance (mcr) rates during insulin clamp studies in patients with non-insulin-dependent diabetes mellitus. Diabetes. 1982;31:396-400

6. Bergstrom J, Hermansen L, Hultman E, Saltin B. Diet, muscle glycogen and physical performance. Acta Physiol. Scand. 1967;71:140-150

7. Bokemark L, Froden A, Attvall S, Wikstrand J, Fagerberg B. The euglycemic hyperinsulinemic clamp examination: Variability and reproducibility. Scand J Clin Lab Invest. 2000;60:27-36

8. Frayn KN. Calculation of substrate oxidation rates in vivo from gaseous exchange. J Appl Physiol. 1983;55:628-634

9. Schols AM, Schoffelen PF, Ceulemans H, Wouters EF, Saris WH. Measurement of resting energy expenditure in patients with chronic obstructive pulmonary disease in a clinical setting. JPEN J Parenter Enteral Nutr. 1992;16:364-368

10. Koopman R, Schaart G, Hesselink MK. Optimisation of oil red o staining permits combination with immunofluorescence and automated quantification of lipids. Histochem Cell Biol. 2001;116:63-68

11. Hoeks J, van Herpen NA, Mensink M, Moonen-Kornips E, van Beurden D, Hesselink MK, Schrauwen P. Prolonged fasting identifies skeletal muscle mitochondrial dysfunction as consequence rather than cause of human insulin resistance. Diabetes. 2010;59:2117-2125

12. Kim JY, Hickner RC, Cortright RL, Dohm GL, Houmard JA. Lipid oxidation is reduced in obese human skeletal muscle. Am. J. Physiol. Endocrinol. Metab. 2000;279:E1039-1044

13. Lemmink KAP, Kemper H, de Greef MHG, Rispens P, Stevens M. Reliability of the groningen fitness test for the elderly. Journal of Aging and Physical Activity. 2001;9

14. Schrauwen-Hinderling VB, Hesselink MK, Meex R, van der Made S, Schar M, Lamb H, Wildberger JE, Glatz J, Snoep G, Kooi ME, Schrauwen P. Improved ejection fraction after exercise training in obesity is accompanied by reduced cardiac lipid content. J Clin Endocrinol Metab. 2010;95:1932-1938

15. Defronzo RA, Tobin JD, Andres R. Glucose clamp technique: A method for quantifying insulin secretion and resistance. Am J Physiol. 1979;237:E214-223

16. Schrauwen-Hinderling VB, Hesselink MK, Meex R, van der Made S, Schar M, Lamb H, Wildberger JE, Glatz J, Snoep G, Kooi ME, Schrauwen P. Improved ejection fraction after 
exercise training in obesity is accompanied by reduced cardiac lipid content. $J$ Clin Endocrinol Metab. 2010;95:1932-1938

17. Haemmerle G, Moustafa T, Woelkart G, Buttner S, Schmidt A, van de Weijer T, Hesselink M, Jaeger D, Kienesberger PC, Zierler K, Schreiber R, Eichmann T, Kolb D, Kotzbeck P, Schweiger M, Kumari M, Eder S, Schoiswohl G, Wongsiriroj N, Pollak NM, Radner FP, Preiss-Landl K, Kolbe T, Rulicke T, Pieske B, Trauner M, Lass A, Zimmermann R, Hoefler G, Cinti S, Kershaw EE, Schrauwen P, Madeo F, Mayer B, Zechner R. Atgl-mediated fat catabolism regulates cardiac mitochondrial function via ppar-alpha and pgc-1. Nat Med. 2011;17:1076-1085

18. Balasubramanian R, Gerrard J, Dalla Man C, Firbank MJ, Lane A, English PT, Cobelli C, Taylor R. Combination peroxisome proliferator-activated receptor gamma and alpha agonist treatment in type 2 diabetes prevents the beneficial pioglitazone effect on liver fat content. Diabet. Med. 2010;27:150-156

19. Cree MG, Newcomer BR, Read LK, Sheffield-Moore M, Paddon-Jones D, Chinkes D, Aarsland A, Wolfe RR. Plasma triglycerides are not related to tissue lipids and insulin sensitivity in elderly following ppar-alpha agonist treatment. Mech. Ageing Dev. 2007;128:558-565 



\section{Abstract}

Introduction: Mitochondrial dysfunction, lipid accumulation, insulin resistance and metabolic inflexibility have been implicated in the etiology of type 2 diabetes (T2D), yet their interrelationship remains speculative. We investigated these interrelationships in a group of T2D and obese normoglycemic control subjects.

Methods: 49 non-insulin dependent male T2D patients and 54 male control subjects were enrolled, and a hyperinsulinemic-euglycemic clamp and indirect calorimetry were performed. A muscle biopsy was taken and intramyocellular lipid (IMCL) was measured. In vivo mitochondrial function was measured by $\mathrm{PCr}$ recovery in $30 \mathrm{~T} 2 \mathrm{D}$ patients and 31 control subjects.

Results: Fasting NEFA levels were significantly elevated in T2D patients compared with controls, but IMCL was not different. Mitochondrial function in T2D patients was compromised by $12.5 \%$ ( $p<0.01)$. Whole body glucose disposal (WGD) was higher at baseline and lower after insulin stimulation. Metabolic flexibility ( $\triangle R E R)$ was lower in the type 2 diabetic patients $(0.050 \pm 0.033$ vs. $0.093 \pm 0.050, p<0.01)$. Mitochondrial function was the sole predictor of basal respiratory exchange ratio (RER) $\left(R^{2}=0.18, p<0.05\right)$; whereas WGD predicted both insulin-stimulated $\operatorname{RER}\left(\mathrm{R}^{2}=0.29, \mathrm{p}<0.001\right)$ and metabolic flexibility $\left(R^{2}=0.40, p<0.001\right)$.

Conclusions: These results indicate that defects in skeletal muscle in vivo mitochondrial function in type 2 diabetic patients are only reflected in basal substrate oxidation and highlight the importance of glucose disposal rate as a determinant of substrate utilization in response to insulin. 


\section{Introduction}

Multiple mechanisms have recently emerged as potential contributors to insulin resistance and type 2 diabetes progression, among them metabolic inflexibility, impaired mitochondrial function and intramyocellular lipid accumulation. Type 2 diabetes is characterized by both diminished rates of fatty acid oxidation in the fasting state and the inability to efficiently switch to glucose oxidation in the post-prandial state ${ }^{1}$. This phenomenon has been termed metabolic inflexibility ${ }^{2,3}$. Although the importance of metabolic inflexibility in insulin resistance and type 2 diabetes has been recognized, the underlying mechanisms are poorly understood. A recent study by Galgani et al. ${ }^{4}$ suggested that metabolic inflexibility is merely a reflection of insulin resistance in obesity and type 2 diabetes. The study concluded that diminished glucose disposal rate was the main determinant of blunted metabolic flexibility in individuals with type 2 diabetes. Glucose disposal rate, however, comprises two distinct parts: oxidative and non-oxidative glucose metabolism. Both of these processes are aberrantly affected in type 2 diabetes ${ }^{5,6}$.

Given the prominent role of skeletal muscle in substrate selection, muscle mitochondrial function is likely to have a prominent role in metabolic flexibility. However, the connection between in vivo mitochondrial function, metabolic flexibility and insulin resistance remains unclear.

The relationship between metabolic flexibility and insulin resistance may also be confounded by elevated plasma fatty acids and muscle lipid accumulation. These factors have been suggested to interfere with muscle insulin sensitivity but may also affect mitochondrial function or vice versa. ${ }^{7-12}$

Therefore, the interrelationships among mitochondrial function, metabolic flexibility and lipid metabolism in the setting of insulin resistance and type 2 diabetes warrants more investigation. In the current study, we assembled data from clamp studies performed at our research center between 2004 and 2011 to assess these interrelationships. 


\section{Methods}

\section{Subjects}

The data in the present study is gathered from clamp studies previously performed in our research center ${ }^{13-17}$. The sample is compromised of volunteers (both diabetic and obese) who have participated in training, pharmacological or nutritional intervention studies. Here we have only used baseline data (eg. data collected prior to the intervention). Forty-nine obese non-insulin dependent sedentary male T2D patients and 54 obese sedentary male control subjects were selected. On average, controls and T2D patients had similar BMI and age. Subjects underwent a complete medical history and physical examination including history of cardiovascular, renal and pulmonary disease, cancer and duration of diabetes, routine medical laboratory tests including hematology, a maximal aerobic capacity test with concurrent ECG as previously described ${ }^{18}$ and anthropometry. Body composition was determined using hydrostatic weighing according to Siri et al. ${ }^{19}$. Control subjects had no family history of T2D and underwent a 2-h oral glucose tolerance test according to World Health Organization criteria. T2D patients had well-controlled diabetes ( $\mathrm{HbA} 1 \mathrm{C} 7.1 \pm 0.8 \%$ ) and were on monotherapy with metformin or metformin combined with sulfonylurea. All patients were diagnosed with type 2 diabetes for at least a year and the average duration of type 2 diabetes was $4.1 \pm 2.8$ years. None of the subjects followed a prescribed dietary program. The Maastricht University Medical Ethical Committee approved these studies, and written informed consent was obtained on all subjects prior to screening. The clinical investigations have been conducted according to the principles expressed in the Declaration of Helsinki.

\section{Hyperinsulinemic-euglycemic clamp}

In all subjects a 3-h hyperinsulinemic-euglycemic ( $40 \mathrm{mU} / \mathrm{m}^{2} / \mathrm{min}$ ) clamp was performed according to DeFronzo ${ }^{20}$. One week prior to the clamp, subjects withdrew from their anti-diabetic medication. After an overnight fast, subjects were primed with an infusion of [6,6-2 $\mathrm{H} 2]$ glucose $(0.04 \mathrm{mg} / \mathrm{kg} / \mathrm{min})$ to determine rates of glucose appearance ( $R \mathrm{a}$, or endogenous glucose production, EGP) and disposal ( $R \mathrm{~d}$, or whole body glucose disposal, WGD) as previously described ${ }^{21}$. Basal and insulin-stimulated measurements were performed, including blood sampling and indirect calorimetry. Type 2 diabetic patients were clamped at slightly higher glucose values than the control subjects $(5.7 \pm 0.5 \mathrm{mmol} / \mathrm{L}$ for controls vs. $6.3 \pm 1.2 \mathrm{mmol} / \mathrm{L}$ for the diabetic patients $p<0.01$ ) due to their hyperglycemia at baseline. Muscle biopsies were performed prior to the clamp under local anesthesia (2\% lidocaine) according to the Bergstrom technique ${ }^{22}$. In these biopsies, muscle lipid 
accumulation was assessed histochemically in muscle cross-sections using a modified oil red $\mathrm{O}$ staining for fluorescence microscopy ${ }^{23}$.

\section{Indirect calorimetry}

During the clamp, both during the basal and the insulin stimulated state, oxygen consumption and carbon dioxide production was measured with an automated respiratory gas analyzer using a ventilated hood system (Omnical; IDEE, Maastricht, The Netherlands). The gas analyzer system was calibrated by an alcohol combustion test before every experiment. From oxygen consumption and carbondioxide production levels, whole body glucose oxidation and fat oxidation rates could be calculated using stoichiometric equations according to Frayn ${ }^{24}$ with the assumption that protein oxidation was negligible.

\section{PCr-recovery by ${ }^{31} \mathrm{P}-\mathrm{MRS}$}

In a subgroup of $30 \mathrm{~T} 2 \mathrm{D}$ patients and 31 control subjects $\mathrm{PCr}$-recovery was measured by ${ }^{31} P$-MRS for in vivo mitochondrial function as previously described ${ }^{13}$. The test was performed at least 1 week prior to the clamp. A knee-extension protocol was performed on a custombuilt magnetic resonance compatible ergometer with a pulley system in a 1.5-T whole-body MRI scanner (Intera; Philips Medical Systems, Best, the Netherlands). The knee extension was performed for $5 \mathrm{~min}$ with weight corresponding to $50-60 \%$ of the subject's predetermined maximal knee-extension capacity. Post-exercise PCr kinetics were computed as previously described ${ }^{13}$.

\section{Plasma assays}

Blood collected in tubes containing EDTA was immediately centrifuged and plasma stored at $-80^{\circ} \mathrm{C}$ until assayed. Plasma non-esterified fatty acids (NEFAs) and glucose were measured with enzymatic assays on a Cobas Fara/Mira (NEFA: Wako Nefa C test kit; Wako Chemicals, Neuss, Germany; Glucose: hexokinase method; LaRoche, Basel, Switzerland). Insulin concentration was determined using a radioimmunoassay (Linco Reseach, St. Charles, $\mathrm{MO})$. Isotopic enrichment of plasma glucose was determined by electron ionization gas chromatography-mass spectrometry and expressed as tracer-to-tracee ratio. 


\section{Calculations}

Steele's single-pool non-steady-state equations were used to calculate glucose endogenous glucose production (EGP) and whole body glucose disposal (WGD) ${ }^{25}$. Volume of distribution was assumed to be $0.160 \mathrm{l} / \mathrm{kg}$ for glucose. Non-oxidative glucose disposal (NOGD) was calculated as WGD minus carbohydrate oxidation. Metabolic flexibility was expressed as the change in respiratory exchange ratio $(\triangle R E R)$ from the fasted to the insulin-stimulated state.

\section{Statistics}

Data are reported as means \pm SE. Statistical analyses were performed using SPSS version 16.0.2 for Mac OS X (SPSS Inc., NC, USA) and JMP version 8.0 (SAS, Cary, NC, USA). Differences between groups were analyzed by one-way ANOVA. Since all variables were normally distributed, correlations were performed in a pair wise fashion using the Pearson product moment statistic. To investigate the relationships among metabolic flexibility and mitochondrial function with other metabolic characteristics, we performed stepwise linear regression analyses. Dependent variables were selected based on simple regression analyses and biological importance in the linear regression analysis. Statistical significance was set a priori at $\mathrm{p}<0.05$.

\section{Results}

\section{Basic characteristics:}

The subject characteristics are presented in Table 1. T2D patients and control subjects were comparable for body weight, BMI and age. Body composition was also similar between groups. By definition, fasting plasma glucose levels were significantly higher in T2D patients compared with control subjects $(9.4 \pm 2.0$ vs. $5.9 \pm 0.8 \mathrm{mmol} / \mathrm{L}, \mathrm{p}<0.01)$; however, fasting plasma insulin levels were not different between groups. Fasting plasma NEFA levels were significantly higher in T2D patients (515.3 \pm 173.7 vs. $425.4 \pm 150.5 \mu \mathrm{mol} / \mathrm{L} ; \mathrm{p}<0.01$ ) compared to control subjects. 
Table 1: Subject characteristics

\begin{tabular}{lccc}
\hline & Control subjects (n) & Diabetic patients (n) & P-value \\
\hline Age (years) & $59.8 \pm 4.8(54)$ & $61.2 \pm 3.7(49)$ & NS \\
FM (\%) & $30.6 \pm 6.3(51)$ & $30.7 \pm 5.3(45)$ & NS \\
FFM (kg) & $63.6 \pm 8.5(51)$ & $64.2 \pm 8.1(45)$ & NS \\
BMI (kg/m2) & $29.4 \pm 3.3(54)$ & $29.8 \pm 3.1(49)$ & NS \\
VO $_{2}$ max (ml/min/kg) & $31.3 \pm 6.2(52)$ & $27.3 \pm 5.8(44)$ & $<0.01$ \\
Fasted NEFA (mol/L) & $425.4 \pm 150.5(53)$ & $515.3 \pm 173.7(46)$ & $<0.01$ \\
Fasted Glucose (mmol/L) & $5.9 \pm 0.8(54)$ & $9.4 \pm 2.0(49)$ & $<0.01$ \\
Fasted insulin (mU/ml) & $14.8 \pm 7.4(49)$ & $16.8 \pm 7.5(45)$ & NS \\
HbA1c (\%) & $5.8 \pm 0.3(18)$ & $7.1 \pm 0.8(41)$ & $<0.01$ \\
PCr-recovery t1/2 (s) & $19.8 \pm 4.5(31)$ & $22.3 \pm 6.9(30)$ & $<0.01$ \\
\hline
\end{tabular}

Basal characteristics of type 2 diabetic patients (male only) and BMI-matched controls performed in the fasting state. FM, fat mass; FFM, fat-free mass; BMI, body mass index; $\mathrm{VO}_{2}$ max, maximal aerobic capacity; NEFA, nonesterified free fatty acids; $\mathrm{HbA} 1 \mathrm{c}$, glycosylated hemoglobin. In vivo mitochondrial function is described by $\mathrm{PCr}-$ recovery half-time rates.

\section{Glucose handling is impaired in type 2 diabetic patients}

Oxidative and non-oxidative glucose uptake data are presented in Table 2.

Basal glucose handling: Basal whole body glucose disposal (WGD) was significantly higher in T2D patients compared to control subjects (12.0 \pm 3.9 vs. $9.4 \pm 2.3 \mu \mathrm{mol} / \mathrm{kg} / \mathrm{min}$, $p<0.01)$. This was reflected in a higher fasting glucose oxidation in T2D patients $(8.3 \pm 2.6$ vs. $6.7 \pm 2.2 \mu \mathrm{mol} / \mathrm{kg} / \mathrm{min}, \mathrm{p}<0.05$ ), whereas non-oxidative glucose disposal (NOGD) was not significantly different between groups ( $3.6 \pm 4.6$ vs. $3.2 \pm 3.2 \mu \mathrm{mol} / \mathrm{kg} / \mathrm{min}, \mathrm{p}>0.05)$. Basal endogenous glucose production (EGP), reflecting hepatic glucose output, was higher in T2D patients compared with control subjects $(11.1 \pm 3.2 \mathrm{vs.} 9.4 \pm 2.3 \mu \mathrm{mol} / \mathrm{kg} / \mathrm{min} ; \mathrm{p}<0.01)$.

Glucose handling during insulin stimulation: Insulin-stimulated WGD was significantly lower in T2D patients compared with control subjects $(20.3 \pm 7.5$ vs. $28.8 \pm 9.5 \mu \mathrm{mol} / \mathrm{kg} / \mathrm{min}$, $\mathrm{p}<0.01)$. This was mainly reflected in a reduced non-oxidative glucose uptake during insulin infusion in T2D patients ( $8.4 \pm 6.9$ vs. $15.4 \pm 8.2 \mu \mathrm{mol} / \mathrm{kg} / \mathrm{min}, p<0.01$ ), although glucose oxidation during insulin infusion was also significantly lower in T2D patients $(11.9 \pm 3.3$ vs. $13.7 \pm 3.74 \mu \mathrm{mol} / \mathrm{kg} / \mathrm{min}, \mathrm{p}<0.05)$. EGP was almost completely suppressed during insulin 
infusion in both groups, although T2D patients still had significantly higher EGP during insulin stimulation $(3.3 \pm 2.5$ vs. $1.9 \pm 2.9 \mu \mathrm{mol} / \mathrm{kg} / \mathrm{min}, \mathrm{p}<0.05)$.

Table 2: Hyperinsulinemic-euglycemic clamp and indirect calorimetry

\begin{tabular}{|c|c|c|c|c|}
\hline & & Control subjects (n) & Diabetic patients (n) & P-value \\
\hline \multirow[t]{2}{*}{ Glucose (mmol/L) } & Basal & $5.7 \pm 0.5(54)$ & $9.0 \pm 2.1(49)$ & $<0.01$ \\
\hline & During Ins. Stim. & $5.3 \pm 0.4(54)$ & $6.3 \pm 1.2(49)$ & $<0.01$ \\
\hline \multirow[t]{3}{*}{ NEFA ( $\mu \mathrm{mol} / \mathrm{L})$} & Basal & $425.4 \pm 150.5(53)$ & $515.3 \pm 173.7(46)$ & $<0.01$ \\
\hline & During Ins. Stim. & $97.4 \pm 38.4(54)$ & $148.8 \pm 72.6(49)$ & $<0.01$ \\
\hline & Delta & $328.0 \pm 156.3(54)$ & $366.5 \pm 125.8$ (49) & $<0.01$ \\
\hline \multirow[t]{2}{*}{ Insulin (mU/L) } & Basal & $15.2 \pm 9.2(52)$ & $17.8 \pm 8.4(48)$ & NS \\
\hline & During Ins. Stim. & $108.8 \pm 24.5(52)$ & $107.8 \pm 20.8(48)$ & NS \\
\hline \multirow[t]{2}{*}{$\mathrm{EE}(\mathrm{KJ} / \mathrm{min})$} & Basal & $5.17 \pm 0.58(49)$ & $5.29 \pm 0.70(47)$ & NS \\
\hline & During Ins. Stim. & $4.66 \pm 0.86(49)$ & $4.77 \pm 0.95(48)$ & NS \\
\hline \multirow[t]{3}{*}{ RER } & Basal & $0.79 \pm 0.03(49)$ & $0.82 \pm 0.03(47)$ & $<0.01$ \\
\hline & During Ins. Stim. & $0.89 \pm 0.04(49)$ & $0.87 \pm 0.04(48)$ & $<0.05$ \\
\hline & Delta & $0.093 \pm 0.05(50)$ & $0.050 \pm 0.033(47)$ & $<0.01$ \\
\hline Glucose oxidation & Basal & $6.96 \pm 2.32(49)$ & $8.34 \pm 2.55(46)$ & $<0.05$ \\
\hline \multirow[t]{2}{*}{$(\mu \mathrm{mol} / \mathrm{kg} / \mathrm{min})$} & During Ins. Stim. & $13.69 \pm 3.74(49)$ & $11.97 \pm 3.25(48)$ & $<0.05$ \\
\hline & Delta & $6.74 \pm 3.60(50)$ & $3.63 \pm 2.53(46)$ & $<0.01$ \\
\hline Lipid oxidation & Basal & $1.09 \pm 0.24(49)$ & $1.01 \pm 0.22(47)$ & NS \\
\hline \multirow[t]{2}{*}{$(\mu \mathrm{mol} / \mathrm{kg} / \mathrm{min})$} & During Ins. Stim. & $0.55 \pm 0.25$ (49) & $0.72 \pm 0.22(48)$ & $<0.01$ \\
\hline & Delta & $0.52 \pm 0.26(49)$ & $0.28 \pm 0.20(47)$ & $<0.01$ \\
\hline \multirow[t]{3}{*}{$\mathrm{EGP}(\mu \mathrm{mol} / \mathrm{kg} / \mathrm{min})$} & Basal & $9.42 \pm 2.34(54)$ & $11.06 \pm 3.22(48)$ & $<0.01$ \\
\hline & During Ins. Stim. & $1.96 \pm 2.91(54)$ & $3.27 \pm 2.46(49)$ & $<0.05$ \\
\hline & Delta & $-7.45 \pm 3.96(54)$ & $-7.94 \pm 3.04(48)$ & NS \\
\hline \multirow[t]{3}{*}{ WGD ( $\mu \mathrm{mol} / \mathrm{kg} / \mathrm{min})$} & Basal & $9.65 \pm 2.59(52)$ & $11.95 \pm 3.88(46)$ & $<0.01$ \\
\hline & During Ins. Stim. & $28.75 \pm 9.45(52)$ & $20.33 \pm 7.50(46)$ & $<0.01$ \\
\hline & Delta & $19.86 \pm 8.58(52)$ & $8.37 \pm 7.42(46)$ & $<0.01$ \\
\hline \multirow[t]{3}{*}{ NOGD $(\mu \mathrm{mol} / \mathrm{kg} / \mathrm{min})$} & Basal & $3.19 \pm 3.16(49)$ & $3.62 \pm 4.56(46)$ & NS \\
\hline & During Ins. Stim. & $15.4 \pm 8.15(49)$ & $8.36 \pm 6.88(46)$ & $<0.01$ \\
\hline & Delta & $12.29 \pm 9.12(49)$ & $4.74 \pm 6.80(46)$ & $<0.01$ \\
\hline
\end{tabular}

In the table above the results of the hyperinsulemic-euglycemic clamp and the ventilated hood measurements that were performed at baseline and during insulin stimulation are presented. Results for plasma metabolite levels are shown at baseline (before insulin stimulation) and after 3 hours of insulin infusion (during Ins. Stim.). NEFA, non-esterified free fatty acids; $E E$, energy expenditure; RER, respiratory exchange ratio; EGP, endogenous glucose production; WGD, whole-body glucose disposal; NOGD, non-oxidative glucose disposal. 


\section{Type 2 diabetic patients have a blunted insulin suppression of lipid oxidation compared to controls despite similar IMCL content}

Data on fatty acid handling fasting and during the clamp are presented in Table 2.

Basal lipid handling: As mentioned above, plasma NEFA levels in the fasted state were significantly higher in T2D patients (Table 1, $\mathrm{p}<0.01$ ). Interestingly, intramyocellular lipid content (IMCL) was not significantly different between the two groups ( $1.10 \pm 0.64$ vs. $0.96 \pm$ $0.66 \%$ for T2D patients and control subjects, respectively, $\mathrm{p}=0.52$ ). In the fasted state, basal lipid oxidation was the same in both groups $(1.0 \pm 0.2$ and $1.1 \pm 0.2$ for T2D patients and controls, respectively; $\mathrm{p}>0.05)$.

Lipid handling during insulin stimulation: Although plasma NEFA levels decreased upon insulin stimulation in both groups, they remained significantly elevated in the T2D patients compared to the controls ( $148.8 \pm 72.6$ vs. $97.4 \pm 38.4 \mu \mathrm{mol} / \mathrm{L}, \mathrm{p}<0.01)$. Insulin stimulation significantly decreased lipid oxidation in both groups, although to a lesser extent in the T2D patients compared to controls ( $-0.3 \pm 0.2$ vs. $-0.5 \pm 0.3 \mu \mathrm{mol} / \mathrm{kg} / \mathrm{min}, \mathrm{p}<0.05)$.

EE did not significantly change upon insulin stimulation in the type 2 diabetic patients nor in the obese controls. Basal EE also did not differ between the groups (see table 2).

\section{Type 2 diabetic patients are metabolically inflexible compared with controls}

Respiratory exchange ratios in basal and insulin-stimulated states are presented in Table 2. Fasting respiratory exchange ratio (RER) was significantly higher in the T2D patients $(0.82$ \pm 0.03 vs. $0.79 \pm 0.03, p<0.01)$ and significantly lower upon insulin stimulation $(0.87 \pm 0.04$ vs. $0.89 \pm 0.04, p<0.05$ ) compared with age-and BMI-matched controls. This resulted in a significantly decreased metabolic flexibility $(\triangle R E R)$, i.e. a lower increase in RER from fasting to insulin-stimulated state $(0.050 \pm 0.033$ vs. $0.093 \pm 0.050, p<0.01)$.

\section{Impaired in vivo mitochondrial function in type $\mathbf{2}$ diabetic patients versus age- and BMI- matched controls}

Mean $\mathrm{PCr}$ recovery half-time ( $\mathrm{PCr}-\mathrm{t} 1 / 2)$ was prolonged by $12.5 \%$ in $\mathrm{T} 2 \mathrm{D}$ patients compared with control subjects ( $22.3 \pm 6.9$ vs. $19.8 \pm 4.5$ seconds, respectively; $p<0.01$, Table 1$)$, thus indicating impaired in vivo mitochondrial function in T2D patients. None of the subjects showed significant acidification at the end of the exercise protocol, and the $\mathrm{pH}$ decreased similarly from rest to end-exercise in both groups (end-exercise pH: $7.05 \pm 0.02$ and 7.02 
$\pm 0.03, \mathrm{p}>0.05$; delta-pH $0.07 \pm 0.02$ and $0.08 \pm 0.03, \mathrm{p}>0.05$, in T2D patients and control subjects, respectively; data not shown).

\section{Simple correlations reveal that mitochondrial function and glucose disposal rate are determinants of metabolic flexibility}

To assess the relationship between the distinct parameters, we performed a simple bivariate correlation analysis. This analysis revealed that metabolic flexibility was positively associated with WGD during insulin stimulation $\left(R^{2}=0.17, p<0.01\right)$ and inversely related to in vivo $\mathrm{PCr}$-recovery half-time $\left(\mathrm{PCr}-\mathrm{t}_{1 / 2}, \mathrm{R}^{2}=0.07, \mathrm{p}<0.05\right)$. $\mathrm{PCr}$-recovery rates were not significantly associated with IMCL (data not shown), but they were inversely related to WGD during insulin stimulation in all subjects $\left(R^{2}=0.10, p<0.05\right)$. This effect seemed to be mainly influenced by non-oxidative glucose disposal, as this, in contrast to glucose oxidation, negatively correlated with $\mathrm{PCr}-\mathrm{t}_{1 / 2}\left(\mathrm{R}^{2}=0.07, \mathrm{p}<0.05\right)$.

\section{Rate of glucose disappearance is a good predictor of metabolic flexibility in both type 2 diabetic patients and BMI-matched controls}

To further investigate, whether these simple correlations were not confounded by other parameters, we performed a stepwise linear regression analyses to identify the main factors that predict metabolic flexibility (Table 3). Similar to the findings of Galgani et al. ${ }^{4}$ , insulin-stimulated plasma NEFA levels and whole body glucose disposal rate significantly contributed to the model $\left(R^{2}=0.40, p<0.001\right.$; Model 1, Table 3 \& Figure $\left.1 A\right)$. Whilst PCrrecovery half-time (i.e. in vivo mitochondrial function) was related to metabolic flexibility in simple bivariate correlation analysis, it was not a significant predictor of metabolic flexibility (Model 1, Table 3 \& Figure 1A). 
Table 3: Stepwise Linear regression analyses

\begin{tabular}{|c|c|c|c|c|c|c|}
\hline Model & Dependent variable & Parameters & Significant Predictors & Beta & $\mathbf{R}^{2}$ & P-value \\
\hline 1 & $\begin{array}{l}\text { Metabolic flexibility } \\
\text { (delta RER) }\end{array}$ & $\begin{array}{l}\text { NEFA clamp } \\
\text { WGD clamp } \\
\text { EE clamp } \\
\text { T1/2 PCr } \\
\text { HbA1c } \\
\text { BMI } \\
\text { Age } \\
\text { VO2max }\end{array}$ & $\begin{array}{l}\text { NEFA clamp } \\
\text { WGD clamp }\end{array}$ & $\begin{array}{l}-0.252 \\
0.304\end{array}$ & 0.40 & $<0.001$ \\
\hline 2 & RER basal & $\begin{array}{l}\text { NEFA basal } \\
\text { WGD basal } \\
\text { EE basal } \\
\text { T1/2 PCr } \\
\text { HbA1c } \\
\text { BMI } \\
\text { Age } \\
\text { VO2max }\end{array}$ & $\begin{array}{l}\mathrm{T} 1 / 2 \mathrm{PCr} \\
\text { Age } \\
\mathrm{BMI}\end{array}$ & $\begin{array}{l}-0.284 \\
-0.186 \\
-0.221\end{array}$ & 0.18 & $<0.04$ \\
\hline 3 & RER insulin stimulated & $\begin{array}{l}\text { NEFA clamp } \\
\text { WGD clamp } \\
\text { EE clamp } \\
\text { T1/2 PCr } \\
\text { HbA1c } \\
\text { BMI } \\
\text { Age } \\
\text { VO2max }\end{array}$ & WGD & 0.457 & 0.29 & $<0.001$ \\
\hline 4 & $\mathrm{~T} 1 / 2 \mathrm{PCr}$ & $\begin{array}{l}\text { VO2max } \\
\text { T2D } \\
\text { Age } \\
\text { BMI }\end{array}$ & $\begin{array}{l}\text { VO2max } \\
\text { T2D }\end{array}$ & $\begin{array}{l}-0.516 \\
0.224\end{array}$ & 0.401 & $<0.01$ \\
\hline
\end{tabular}

In the above table the results of the stepwise regression analyses are presented. Indicated in the "Parameters" column are all variables included in the model, and in the "Significant Predictors" column are all variables that were significant after stepwise regression. In the Beta column, the beta value for each (significant) predictor in the model

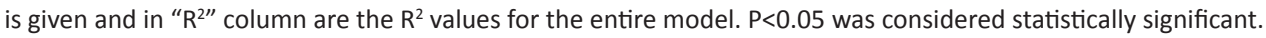




\section{The individual components of metabolic flexibility (basal and insulin-stimulated respiratory exchange ratio) are defined by different metabolic parameters}

Next, we sought to determine the predictors of the individual components of metabolic flexibility, fasting RER under basal conditions and insulin-stimulated RER. Interestingly, in vivo mitochondrial function, determined by PCr-recovery half-time, was the only significant predictor of basal RER ( $R^{2}=0.18, p<0.04$; Model 2, Table 3 \& Figure 1B). As expected, only whole body glucose disposal rate during insulin stimulation (WGD) was a significant predictor of insulin-stimulated RER ( $R^{2}=0.29, p<0.001$; Model 3, Table 3 \& Figure $1 C$ ). Plasma NEFA levels did not significantly contribute to the prediction of insulin-stimulated RER.

Figure 1: Predicted relationships from stepwise linear regression analyses

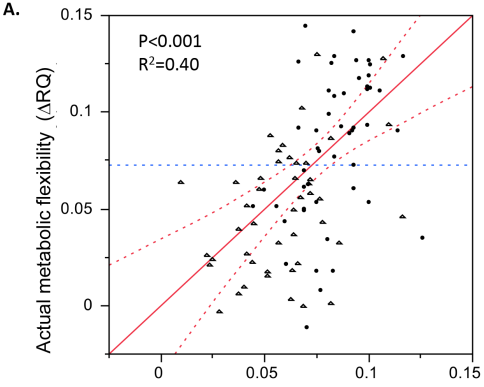

Predicted metabolic flexibility by FFA and WGD $(\triangle \mathrm{RQ}$ model 1)

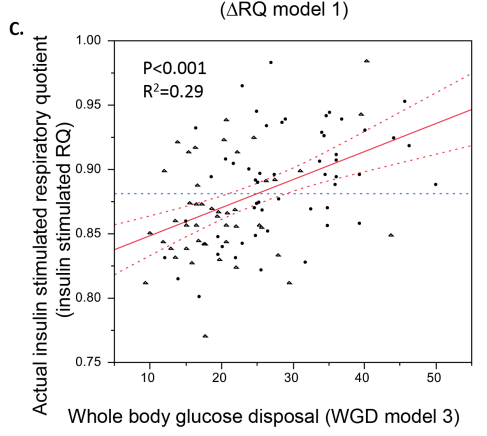

B.
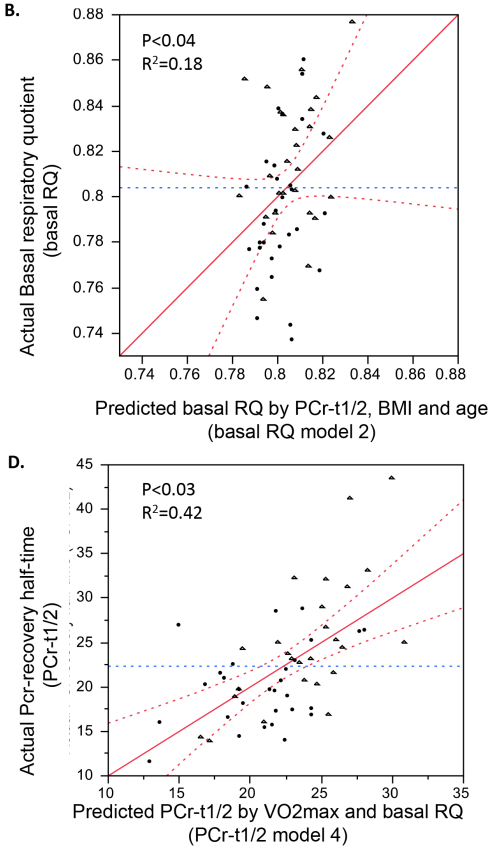

On the $x$-axis you can find the values predicted by the models by the different parameters in the model, on the $y$-axis you can find the actual value as measured in the study. The results were plotted for the following models: (A) Model 1, relationship between actual metabolic flexibility ( $\triangle R E R)$ and the metabolic flexibility predicted by Model 1 (by plasma NEFA and WGD during insulin stimulation), (B) Model 2; relationship between actual basal respiratory exchange ratio (basal RER) and the basal RER predicted by Model 2 (by plasma PCr-recovery half-time, $\mathrm{BMI}$ and age), (C) Model 3; relationship between insulin-stimulated respiratory exchange ratio (ins. stim. RER) and the insulin-stimulated RER predicted by Model 3 (by WGD during insulin stimulation), (D) Model 4; relationship between actual PCr-recovery half-time and PCr-recovery half-time predicted by Model 4 (by basal RER and VO2max). 


\section{Discussion}

In our present study, we confirm that type 2 diabetic patients are metabolically inflexible and have reduced in vivo mitochondrial function. Controversy exists regarding the relationship between mitochondrial function and type 2 diabetes.

In this study in vivo mitochondrial function was measured by assessing the half-time of $\mathrm{PCr}$ resynthesis after exercise with ${ }^{31} \mathrm{P}-\mathrm{MRS}$. The PCr-recovery kinetics reflect the capacity to oxidatively generate ATP, and depends among others on intrinsic mitochondrial function, mitochondrial density and perfusion, thereby reflecting muscle oxidative capacity. Ex vivo assays, like high resolution respirometry, mitochondrial density assays and measurements of ROS production more accurately assess the sources of the decreased capacity. To evaluate to what extent mitochondrial function is determining metabolic flexibility, a measurement of the in vivo situation is warranted.

It has been speculated that mitochondrial abnormalities drive an impaired lipid oxidation with augmented lipid storage in skeletal muscle as a consequence. This lipid storage and defects in beta-oxidation have been shown to parallel a decrease in insulin sensitivity and metabolic flexibility via impairment of insulin signaling pathways and a subsequent decline in glucose uptake by lipotoxic intermediates ${ }^{26-31}$. Indeed we did find an impaired mitochondrial capacity in our type 2 diabetic patients. However, the reduction in insulin sensitivity and mitochondrial function as observed in our type 2 diabetic patients was independent of intramyocellular lipid content, which was similar between the two groups. Although this could suggest that intramyocellular lipid storage may not be a main determinant of insulin sensitivity when BMI and age matched obese and T2D patients are compared, we cannot rule out the possibility that lipid intermediates were different between groups. Also we did not do any ex vivo assays to more accurately assess the source of the decreased capacity. Hence several questions remain which cannot be answered by the correlative analysis performed in this study.

However, we did find a relationship between in vivo mitochondrial capacity and metabolic flexibility. The relationship of mitochondrial capacity with metabolic flexibility disappeared when we added whole body glucose disposal to the model. Upon a more thorough analysis of the different components of metabolic flexibility, we found that in vivo mitochondrial function was the single predictor of basal RER. In contrast, insulin-stimulated RER was mainly determined by glucose disposal rate and not by in vivo mitochondrial function. This suggests that reduced mitochondrial function does not negatively impact the ability of the skeletal muscle to switch substrates; rather it is primarily responsible for basal substrate utilization. Moreover, substrate oxidation during insulin stimulation seems to be mainly 
determined by substrate availability, such as insulin-stimulated glucose uptake, which is in agreement with the work by Galgani and colleagues ${ }^{4}$.

Consistent with the finding that insulin-stimulated RER is mainly dependent on glucose disposal rates, Kelley et al. ${ }^{2}$ demonstrated that in obese patients after weight loss, both insulin sensitivity and insulin-stimulated RER normalized to the level of their lean controls. While it is well known that weight loss does not improve mitochondrial function ${ }^{32,33}$, in the study of Kelly et al, Baseline RER could not be restored after weight loss. This might fit with our theory that basal RER is mainly dependent on mitochondrial function.

Besides glucose disposal rates, also plasma NEFA levels, seemed an important determinant of metabolic flexibility. Both whole body glucose disposal and plasma NEFA levels during insulin stimulation significantly contributed to the prediction of metabolic flexibility, which also supports the findings of Galgani et al ${ }^{4}$. Plasma free fatty acid levels were positively associated with fatty acid oxidation rates (both in the fasted $\left(R^{2}=0.047, p<0.05\right)$ and in the insulin-stimulated state $\left.\left(R^{2}=0.047, p<0.05\right)\right)$; therefore it might be plausible that the influence on metabolic flexibility is primarily substrate driven, as proposed by the Randle cycle theory ${ }^{28}$.

It might be speculated that reduced mitochondrial function in diabetic patients is influenced by differences in physical activity levels between the groups ${ }^{34,35}$. Indeed, VO2max (reflecting physical fitness) is reduced in the diabetic patients. We addressed this issue by correcting for maximal aerobic capacity (VO2max) and found that the difference in in vivo mitochondrial function remained significantly reduced in the type 2 diabetic patients compared to their controls (data not shown). This suggests that this defect is more than just a difference in training status in these patients. Even so, one can question why in vivo mitochondrial function measured by PCr recovery - reflecting ATP synthetic capacity - would affect basal substrate oxidation. Thus, a reduced capacity to synthesize ATP could be due to increased mitochondrial uncoupling, which might be compensated by increased oxidation in the basal state. However, it should be noted that uncoupling only accounts for a small part of the total oxidation rates in skeletal muscle, and as energy expenditure was not significantly different, the effect of muscle mitochondrial uncoupling on the whole body oxidation rates in this study probably is small. Actually, if anything, earlier results indicated that mitochondrial uncoupling would be decreased in diabetes rather than increased ${ }^{31,36-39}$. However, more studies are needed to understand how mitochondrial function can affect basal substrate oxidation. 
In conclusion, the current study advances the concept that mitochondrial function is a main determinant of basal substrate handling, whereas glucose disposal rate is the primary determinant of insulin-stimulated substrate handling. Furthermore, plasma free fatty acid levels are significantly elevated in type 2 diabetes when compared to obese subjects with similar age, BMI and fat mass, and may contribute to the defects consistently observed in metabolic flexibility in individuals with type 2 diabetes.

\section{Acknowledgements}

P.S. is supported by a VICl Research Grant for innovative research from the Netherlands Organization for Scientific Research (Grant 918.96.618). T.v.d.W. was supported by the Center for Translational Molecular Medicine (CTMM) project PREDICCt (Grant 01C-104) and the Netherlands Heart Foundation, Dutch Diabetes Research Foundation, and Dutch Kidney Foundation. V.S. is supported by a VENI research grant for innovative research from the Netherlands Organization for Scientific Research (Grant 916.11.136). The funders had no role in study design, data collection and analysis, decision to publish, or preparation of the manuscript. 


\section{References}

1. Kelley DE, Mandarino LJ. Fuel selection in human skeletal muscle in insulin resistance: A reexamination. Diabetes. 2000;49:677-683

2. Kelley DE, Goodpaster B, Wing RR, Simoneau JA. Skeletal muscle fatty acid metabolism in association with insulin resistance, obesity, and weight loss. Am J Physiol. 1999;277:E11301141

3. Corpeleijn E, Saris WH, Blaak EE. Metabolic flexibility in the development of insulin resistance and type 2 diabetes: Effects of lifestyle. Obes Rev. 2009;10:178-193

4. Galgani JE, Heilbronn LK, Azuma K, Kelley DE, Albu JB, Pi-Sunyer X, Smith SR, Ravussin E. Metabolic flexibility in response to glucose is not impaired in people with type 2 diabetes after controlling for glucose disposal rate. Diabetes. 2008;57:841-845

5. Golay A, DeFronzo RA, Ferrannini E, Simonson DC, Thorin D, Acheson K, Thiebaud D, Curchod $B$, Jequier E, Felber JP. Oxidative and non-oxidative glucose metabolism in non-obese type 2 (non-insulin-dependent) diabetic patients. Diabetologia. 1988;31:585-591

6. Golay A, Defronzo RA, Thorin D, Jequier E, Felber JP. Glucose disposal in obese non-diabetic and diabetic type ii patients. A study by indirect calorimetry and euglycemic insulin clamp. Diabete Metab. 1988;14:443-451

7. Kelley DE, Mokan M, Simoneau JA, Mandarino LJ. Interaction between glucose and free fatty acid metabolism in human skeletal muscle. J Clin Invest. 1993;92:91-98

8. Perseghin G, Scifo P, De Cobelli F, Pagliato E, Battezzati A, Arcelloni C, Vanzulli A, Testolin G, Pozza G, Del Maschio A, Luzi L. Intramyocellular triglyceride content is a determinant of in vivo insulin resistance in humans: A $1 \mathrm{~h}-13 \mathrm{c}$ nuclear magnetic resonance spectroscopy assessment in offspring of type 2 diabetic parents. Diabetes. 1999;48:1600-1606

9. Jacob S, Machann J, Rett K, Brechtel K, Volk A, Renn W, Maerker E, Matthaei S, Schick F, Claussen CD, Haring HU. Association of increased intramyocellular lipid content with insulin resistance in lean nondiabetic offspring of type 2 diabetic subjects. Diabetes. 1999;48:11131119

10. Eckel RH, Grundy SM, Zimmet PZ. The metabolic syndrome. Lancet. 2005;365:1415-1428

11. Griffin ME, Marcucci MJ, Cline GW, Bell K, Barucci N, Lee D, Goodyear LJ, Kraegen EW, White MF, Shulman GI. Free fatty acid-induced insulin resistance is associated with activation of protein kinase $\mathrm{c}$ theta and alterations in the insulin signaling cascade. Diabetes. 1999;48:1270-1274

12. Dresner A, Laurent D, Marcucci M, Griffin ME, Dufour S, Cline GW, Slezak LA, Andersen DK, Hundal RS, Rothman DL, Petersen KF, Shulman GI. Effects of free fatty acids on glucose transport and irs-1-associated phosphatidylinositol 3-kinase activity. J Clin Invest. 1999;103:253-259

13. Schrauwen-Hinderling VB, Kooi ME, Hesselink MK, Jeneson JA, Backes WH, van Echteld CJ, van Engelshoven JM, Mensink M, Schrauwen P. Impaired in vivo mitochondrial function but similar intramyocellular lipid content in patients with type 2 diabetes mellitus and bmimatched control subjects. Diabetologia. 2007;50:113-120

14. Schrauwen-Hinderling VB, Mensink M, Hesselink MK, Sels JP, Kooi ME, Schrauwen P. The insulin-sensitizing effect of rosiglitazone in type 2 diabetes mellitus patients does not require improved in vivo muscle mitochondrial function. J Clin Endocrinol Metab. 2008;93:29172921 
15. van Herpen NA, Schrauwen-Hinderling VB, Schaart G, Mensink RP, Schrauwen P. Three weeks on a high-fat diet increases intrahepatic lipid accumulation and decreases metabolic flexibility in healthy overweight men. J Clin Endocrinol Metab. 2011

16. Meex RC, Schrauwen-Hinderling VB, Moonen-Kornips E, Schaart G, Mensink M, Phielix E, van de Weijer T, Sels JP, Schrauwen P, Hesselink MK. Restoration of muscle mitochondrial function and metabolic flexibility in type 2 diabetes by exercise training is paralleled by increased myocellular fat storage and improved insulin sensitivity. Diabetes. 2010;59:572579

17. Phielix E, Schrauwen-Hinderling VB, Mensink M, Lenaers E, Meex R, Hoeks J, Kooi ME, Moonen-Kornips E, Sels JP, Hesselink MK, Schrauwen P. Lower intrinsic adp-stimulated mitochondrial respiration underlies in vivo mitochondrial dysfunction in muscle of male type 2 diabetic patients. Diabetes. 2008;57:2943-2949

18. Kuipers H, Verstappen FT, Keizer HA, Geurten P, van Kranenburg G. Variability of aerobic performance in the laboratory and its physiologic correlates. Int J Sports Med. 1985;6:197201

19. Siri WE. The gross composition of the body. Adv Biol Med Phys. 1956;4:239-280

20. DeFronzo RA, Tobin JD, Andres R. Glucose clamp technique: A method for quantifying insulin secretion and resistance. Am J Physiol. 1979;237:E214-223

21. Mensink M, Blaak EE, van Baak MA, Wagenmakers AJ, Saris WH. Plasma free fatty acid uptake and oxidation are already diminished in subjects at high risk for developing type 2 diabetes. Diabetes. 2001;50:2548-2554

22. Bergstrom J, Hermansen L, Hultman E, Saltin B. Diet, muscle glycogen and physical performance. Acta Physiol Scand. 1967;71:140-150

23. Koopman R, Schaart G, Hesselink MK. Optimisation of oil red o staining permits combination with immunofluorescence and automated quantification of lipids. Histochem Cell Biol. 2001;116:63-68

24. Frayn KN. Calculation of substrate oxidation rates in vivo from gaseous exchange. J App/ Physiol. 1983;55:628-634

25. Steele R. Influences of glucose loading and of injected insulin on hepatic glucose output. Ann $N$ Y Acad Sci. 1959;82:420-430

26. Boden G, Chen X, Ruiz J, White JV, Rossetti L. Mechanisms of fatty acid-induced inhibition of glucose uptake. J Clin Invest. 1994;93:2438-2446

27. McGarry JD. What if minkowski had been ageusic? An alternative angle on diabetes. Science (New York, N.Y. 1992;258:766-770

28. Randle PJ, Garland PB, Hales CN, Newsholme EA. The glucose fatty-acid cycle. Its role in insulin sensitivity and the metabolic disturbances of diabetes mellitus. Lancet. 1963;1:785789

29. DeFronzo RA. Dysfunctional fat cells, lipotoxicity and type 2 diabetes. Int J Clin Pract Suppl. 2004:9-21

30. Kashyap S, Belfort R, Gastaldelli A, Pratipanawatr T, Berria R, Pratipanawatr W, Bajaj M, Mandarino L, DeFronzo R, Cusi K. A sustained increase in plasma free fatty acids impairs insulin secretion in nondiabetic subjects genetically predisposed to develop type 2 diabetes. Diabetes. 2003;52:2461-2474

31. Schrauwen P, Schrauwen-Hinderling V, Hoeks J, Hesselink MK. Mitochondrial dysfunction and lipotoxicity. Biochim Biophys Acta. 2010;1801:266-271 
32. Rabol R, Svendsen PF, Skovbro M, Boushel R, Haugaard SB, Schjerling P, Schrauwen P, Hesselink MK, Nilas L, Madsbad S, Dela F. Reduced skeletal muscle mitochondrial respiration and improved glucose metabolism in nondiabetic obese women during a very low calorie dietary intervention leading to rapid weight loss. Metabolism. 2009;58:1145-1152

33. Snel M, Gastaldelli A, Ouwens DM, Hesselink MK, Schaart G, Buzzigoli E, Frolich M, Romijn JA, Pijl $\mathrm{H}$, Meinders AE, Jazet IM. Effects of adding exercise to a 16-week very low-calorie diet in obese, insulin-dependent type 2 diabetes mellitus patients. J Clin Endocrinol Metab. 2012;97:2512-2520

34. Galgani JE, Johannsen NM, Bajpeyi S, Costford SR, Zhang Z, Gupta AK, Ravussin E. Role of skeletal muscle mitochondrial density on exercise-stimulated lipid oxidation. Obesity (Silver Spring). 2012;20:1387-1393

35. Rabol R, Boushel R, Dela F. Mitochondrial oxidative function and type 2 diabetes. Appl Physiol Nutr Metab. 2006;31:675-683

36. Boushel R, Gnaiger E, Schjerling P, Skovbro M, Kraunsoe R, Dela F. Patients with type 2 diabetes have normal mitochondrial function in skeletal muscle. Diabetologia. 2007;50:790796

37. Han DH, Nolte LA, Ju JS, Coleman T, Holloszy JO, Semenkovich CF. Ucp-mediated energy depletion in skeletal muscle increases glucose transport despite lipid accumulation and mitochondrial dysfunction. Am J Physiol Endocrinol Metab. 2004;286:E347-353

38. Hoeks J, Hesselink MK, Russell AP, Mensink M, Saris WH, Mensink RP, Schrauwen P. Peroxisome proliferator-activated receptor-gamma coactivator-1 and insulin resistance: Acute effect of fatty acids. Diabetologia. 2006;49:2419-2426

39. Mogensen M, Sahlin K, Fernstrom M, Glintborg D, Vind BF, Beck-Nielsen H, Hojlund K. Mitochondrial respiration is decreased in skeletal muscle of patients with type 2 diabetes.

Diabetes. 2007;56:1592-1599 


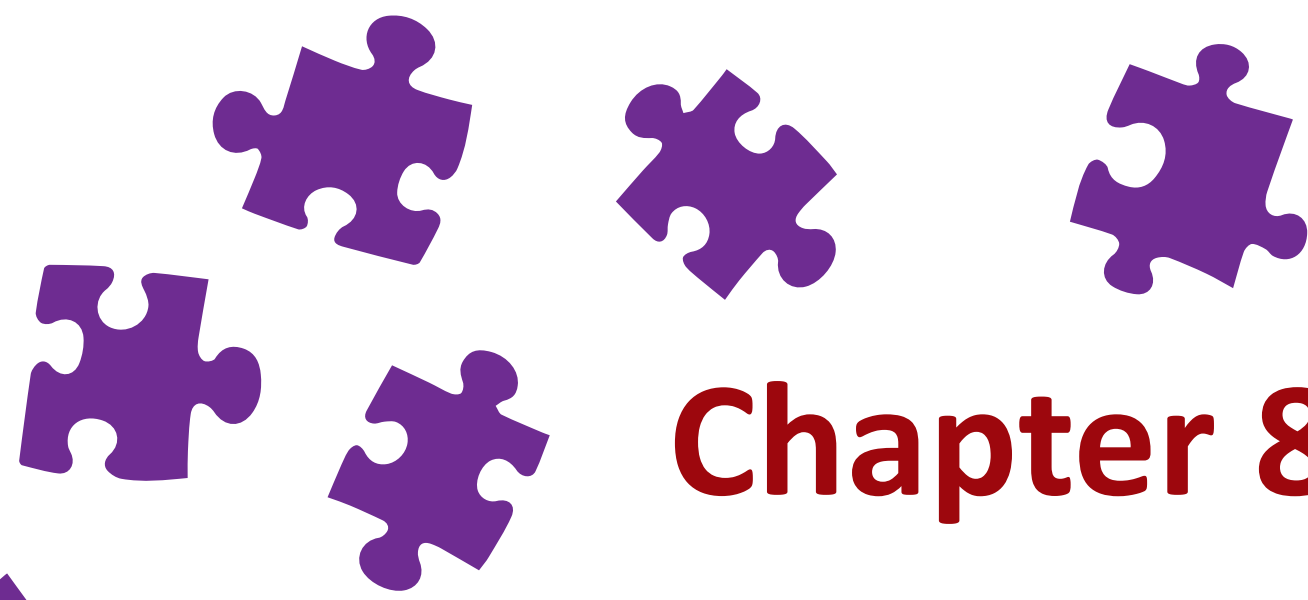

Chapter 8

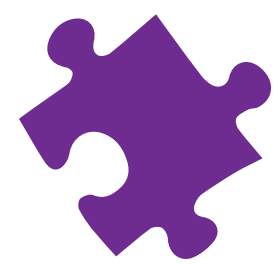

\section{Exercise-induced modulation of cardiac lipid content in healthy lean young men}

Lena Bilet ${ }^{1,6}$, Tineke van de Weijer ${ }^{1,6}$, Matthijs K.C. Hesselink ${ }^{2,6}$, Jan F. C. Glatz ${ }^{3,7}$, Hildo J. Lamb ${ }^{4}$, Joachim Wildberger ${ }^{5}$, Marianne E. Kooi ${ }^{5,7}$, Patrick Schrauwen ${ }^{1,5}$, Vera B. Schrauwen-Hinderling 5,6

${ }^{1}$ Department of Human Biology, Maastricht University Medical Center, The Netherlands, ${ }^{2}$ Department of Human Movement Sciences, Maastricht University Medical Center, The Netherlands, ${ }^{3}$ Department of Molecular Genetics, Maastricht University Medical Center, The Netherlands, ${ }^{4}$ Department of Radiology, Leiden University Medical Center, Leiden, The Netherlands, ${ }^{5}$ Department of Radiology, Maastricht University Medical Center, The Netherlands, ${ }^{6}$ School for Nutrition, Toxicology and Metabolism, Maastricht University Medical Center,

The Netherlands, ${ }^{7}$ Cardiovascular Research Institute Maastricht, Maastricht University Medical Center, The

Netherlands. 


\section{Abstract}

Background and aims: Cardiac lipid accumulation is associated with decreased cardiac function and energy status (PCr/ATP). It has been suggested that elevated plasma fatty acid (FA) concentrations are responsible for cardiac lipid accumulation. Therefore, the aim of the present study was to investiagte if elevating plasma FA concentrations by exercise results in an increased cardiac lipid content, and if this influences cardiac function and energy status. Materials and methods: Eleven male subjects (age: $25.4 \pm 1.1 \mathrm{y}, \mathrm{BMI}: 23.6 \pm 0.8 \mathrm{~kg} / \mathrm{m}^{2}$ ) performed a $2 \mathrm{~h}$ cycling protocol, once while staying fasted, and once while ingesing glucose, to create a state of high verus low plasma FA concentrations, respectively. Cardiac lipid conetent was measured by Proton Magnetic Resonance Spectroscopy ( $\left.{ }^{1} \mathrm{H}-\mathrm{MRS}\right)$ at baseline, directly after exercise and again $4 \mathrm{~h}$ post exercise, together with systolic function (by multislice cine-MRI) and cardiac energy status (by ${ }^{31} \mathrm{P}-\mathrm{MRS}$ ).

Results: Plasma FA concentrations were increased three-fold during exercise and ninefold during recovery in the fasted state compared with the glucose-fed state $(p<0.01)$. Cardiac lipid content was elevated at the end of the fasted test day (from $0.26 \pm 0.04$ to $0.44 \pm 0.04 \%, p=0.003$ ), while it did not change with glucose supplementation (from $0.32 \pm 0.03 \%$ to $0.26 \pm 0.05 \%, p=0.272$ ). Furthermore, PCr/ATP was decreased by $32 \%$ in the high plasma FA state compared with the low FA state $(n=6, P=0.014)$. However, in the high FA state, the ejection fraction $4 \mathrm{~h}$ post exercise was higher compared with the low FA state ( $63 \pm 2 \%$ vs $59 \pm 2 \%, p=0.018$ ).

Conclusion: Elevated plasma FA concentrations, induced by exercise in the fasted state, lead to increased cardiac lipid content but do not acutely hamper systolic function. Although the lower cardiac energy status is in line with a lipotoxic action of cardiac lipid content, a causal relationship cannot be proven. 


\section{Introduction}

Hospitalization for congestive heart failure is increasing world wide ${ }^{1}$. This increase is associated with the increasing prevalence of obesity ${ }^{2,3}$, which is an independent risk factor for the development of heart failure ${ }^{4}$. Obesity is associated with excessive storage of lipids (triglycerides) not only in adipose tissue but also in skeletal muscle, the liver and the heart. It has been suggested that lipid accumulation in cardiac tissue may have lipotoxic effects, thereby hampering cardiac function and predisposing to cardiomyopathy and heart failure. Cardiac lipotoxicity is well documented in both obese ${ }^{5,6}$ and lean animal models with exaggerated storage of fat in cardiac muscle induced by targeted over expression of genes involved in lipid delivery and synthesis in the myocardium ${ }^{7-11}$, but direct evidence for cardiac lipotoxicity in humans is scarce. However, proton magnetic resonance spectroscopy ( $\left.{ }^{1} \mathrm{H}-\mathrm{MRS}\right)$ has recently been proven reliable for non-invasive in vivo investigation of cardiac tissue and enables human interventional studies ${ }^{12,13}$. Such studies have indicated that cardiac lipid accumulation is elevated in conditions of increased circulating fatty acid (FA) concentrations, such as obesity ${ }^{14}$, a very low calorie $\operatorname{diet}^{15}$ and fasting ${ }^{16}$, and circulating FA concentrations were found to correlate with cardiac lipid content ${ }^{14}$. These data suggest that the cardiac uptake of FA is not tightly matched to oxidational needs but rather follows circulating FA concentrations. In addition, it has been shown that high plasma FA concentrations correlate negatively with the energy status of the heart ${ }^{17}$, and the latter has a strong prognostic value in heart failure patients and might be an early marker of disturbed cardiac metabolism ${ }^{18}$. In that respect, it is interesting to examine the effect of acute exercise on cardiac lipid content and function. Acute exercise - when performed in the fasting state - is associated with a 3 -fold elevation of plasma FA concentrations ${ }^{19}$. However, when acute exercise is performed while providing carbohydrate beverages, the increase in FA can be completely blunted. It could be hypothesized that cardiac lipid content will increase during exercise performed in the fasting state, due to elevated plasma FA concentrations. On the other hand, lipid content in skeletal muscle can be lowered by prolonged acute exercise ${ }^{20}$ even despite high circulatory FA concentrations, due to increased fat oxidation to fuel physical activity. We have recently shown that prolonged endurance training decreased cardiac lipid content in parallel with improvements in cardiac function ${ }^{21}$. Thus, it can also be hypothesized that cardiac fat content will decrease during exercise due to an increased oxidation of FA needed to meet the increased cardiac work. Therefore, comparing the effect of acute exercise with high versus low circulating FA concentrations will allow us to test the hypothesis that physiologically-induced elevation of plasma FA concentrations lead to an unrestricted uptake of FA in cardiac tissue, subsequent accumulation of intracardiac fat, and an ultimate reduction in cardiac function and cardiac energy status. To this end, we employed ${ }^{1} \mathrm{H}-\mathrm{MRS}$ to 
determine cardiac lipid content before and after exercise as well as four hours post exercise. $\mathrm{MRI}$ and ${ }^{31} \mathrm{P}-\mathrm{MRS}$ was employed four hours post exercise to determine cardiac function and cardiac energy status, respectively. 


\section{Materials and Methods}

\section{Subjects}

Eleven healthy lean, untrained young men participated in this study. Subjects' characteristics are shown in table 1 . None of the subjects participated in competitive sports and subjects with unstable weight ( $>3 \mathrm{~kg}$ change in preceding six months) were excluded from the study. The institutional medical ethical committee approved the study and written informed consent was obtained from all participants.

\section{Table 1: Subject Characteristics}

\begin{tabular}{ll}
\hline Subject & Mean \pm SE \\
\hline Age, yr & $25.4 \pm 1.1$ \\
Height, $\mathrm{m}$ & $1.79 \pm 0.02$ \\
Weight, kg & $75.8 \pm 3.5$ \\
$\mathrm{BMI}, \mathrm{kg} / \mathrm{m}^{2}$ & $23.6 \pm 0.8$ \\
Fat \% & $18.3 \pm 2.0$ \\
$\mathrm{VO}_{2 \mathrm{max}} / \mathrm{kg}, \mathrm{ml} \times \mathrm{min}^{-1} \times \mathrm{kg}^{-1}$ & $45.9 \pm 2.2$ \\
\hline
\end{tabular}

\section{Study protocol}

Before the start of the study, body composition and maximal aerobic capacity was determined in all subjects. The experimental trial comprised two separate test days. Subjects refrained from physical activity two days prior to the test days. Furthermore, subjects were instructed to consume a standardized meal the evening prior to the test days and stay fasted from 10 pm onwards. On the test days, subjects came to the laboratory at 7:00 am after an overnight fast. Cardiac lipid content was investigated by ${ }^{1} \mathrm{H}-\mathrm{MRS}$. After this, a teflon canula was inserted into an antecubital vein for sampling of blood. Immediately after taking the first blood sample, subjects ingested $1.4 \mathrm{~g} / \mathrm{kg}$ body weight of glucose (dissolved in water to a $20 \%$ solution and flavoured with $1 \mathrm{ml}$ lemon juice) or the same amount of water on the test day in fasted state. Next, subjects were guided to another lab where they started exercising at $50 \%$ of their maximal power output ( $t=0$ minute). The exercise period lasted for two hours and during exercise blood samples were taken and substrate oxidation was measured by indirect calorimetry for 15 minutes (Omnical, Maastricht, The Netherlands) every 30 minutes (at $t=30,60,90$ and 120 minutes). Immediately after cessation of the two hours of exercise, a second ${ }^{1} \mathrm{H}$-MRS measurement was performed. Subsequently, subjects bed-rested 
for three hours and a third ${ }^{1} \mathrm{H}-\mathrm{MRS}$ scan together with additional measurements of ejection fraction (by CINE-MRI) and cardiac energy status (by ${ }^{31} \mathrm{P}$ MRS) were started four hours after exercise. During this post exercise period, blood samples were taken and substrate oxidation measured for 15 minutes every hour (at $\mathrm{t}=180,240,300$ and 360 minutes). The experimental design is depicted in figure 1.

The entire protocol was performed twice: once with the ingestion of glucose $(0.35 \mathrm{~g} / \mathrm{kg}$ body weight at $\mathrm{t}=-15,30,60,90,180,240,300$ and 360 ) after the initial bolus of $1.4 \mathrm{~g} / \mathrm{kg}$ of body weight and once in the fasted state with ingestion of equal amounts of water. Both test days were separated by at least one week and were performed in random order. On a separate day a reference measurement of cardiac function without prior exercise was performed in the morning after an overnight fast.

\section{Figure 1: Experimental design of the study}

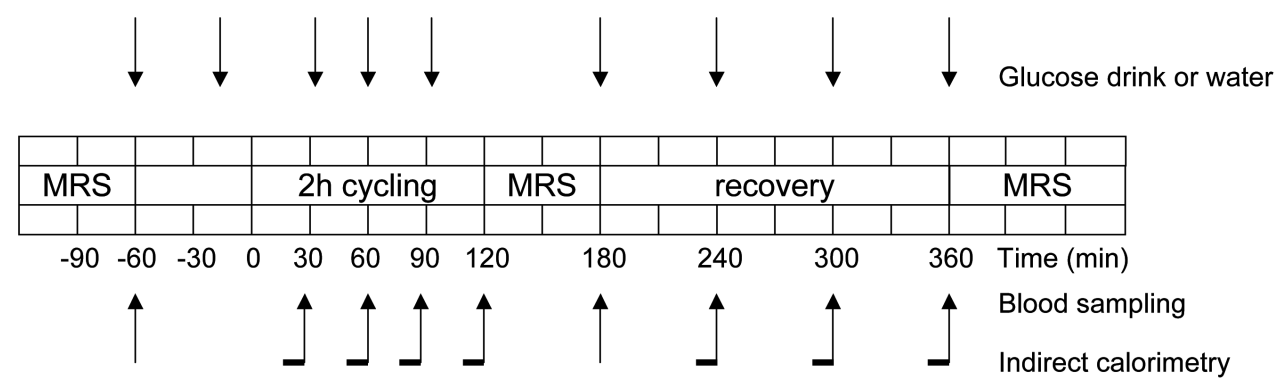

All subjects performed the protocol two times, one time in the fasted state consuming water and one time with glucose supplementation. MRS: cardiac magnetic resonance spectroscopy.

\section{Maximal oxygen uptake (whole body oxidative capacity)}

A routine incremental cycling test was used to determine maximal aerobic capacity as described previously ${ }^{22}$. Briefly, after a warming-up period of $5 \mathrm{~min}$, the intensity was increased every 2.5 min until exhaustion. Oxygen consumption was measured continuously throughout the test using indirect calorimetry (Omnical, Maastricht, The Netherlands) to determine $\mathrm{VO}_{2}$ max. 


\section{Hydrostatic weighing}

Hydrostatic weighing with simultaneous measurement of lung volume was used to determine body composition in the morning in the fasted state. The equation of $\mathrm{Siri}^{23}$ was used to calculate fat percentage, fat mass and fat-free mass.

\section{Blood sample analysis}

Blood samples were collected in EDTA-containing tubes and immediately centrifuged at high speed and frozen in liquid nitrogen and thereafter stored at $-80^{\circ} \mathrm{C}$ until assayed. Plasma fatty acids, triglycerides and glucose were measured with enzymatic assays automated on a Cobas Fara/Mira (FA: Wako Nefa C test kit; Wako Chemicals, Neuss, Germany) (glucose: hexokinase method; Roche, Basel, Switzerland) (triglycerides: ABX Pentra CP reagens, Horiba ABX Diagnostics, Montpellier, France) (glycerol: Enzytec ${ }^{\mathrm{TM}}$ glycerol kit, R-Biopharm, Germany).

\section{MRI}

Cardiac CINE-MRI examinations were performed on a whole body MRI-scanner (Intera, 1.5 T, Philips Healthcare, Best, The Netherlands) as reported previously ${ }^{13}$. At the end of the test day, slices covering the whole left ventricle were acquired in 24 heart phases, to determine parameters of systolic function. During the other measurements, only four slices were acquired, which was sufficient to guide spectroscopy.

\section{Image processing}

All images were analyzed quantitatively and ten images were examined for inter-observer reliability using dedicated software (CAAS, Pie Medical Imaging, Maastricht, The Netherlands) to determine end diastolic and end systolic volumes, left ventricular stroke volume, ejection fraction, cardiac output and cardiac index.

\section{${ }^{1} \mathrm{H}$ MRS}

Cardiac lipid content was determined in vivo by ${ }^{1} \mathrm{H}$ MRS as reported previous ${ }^{21}$. Respiratory gating and tracking was performed with a pencil beam navigator placed on the diaphragm ${ }^{13}$. Chemical shift selective (CHESS) water suppression was performed to acquire spectra of the lipid metabolites using a series of 32 spectra ( $n=2$ each), resulting in a total of 64 acquisitions. To acquire a reference spectrum of the unsuppressed water peak in the same volume of interest, the acquisition was repeated, with the CHESS pulse off resonance, using a series of 
12 spectra ( $n=2$ each), resulting in a total of 24 acquisitions. From eleven subjects two had to be excluded from the cardiac lipid content analysis due to poor quality of spectra.

\section{${ }^{1} \mathrm{H}-\mathrm{MRS}$ spectral post processing}

Post processing of the spectra was performed with the jMRUI software ${ }^{24}$. All ${ }^{1} \mathrm{H} \mathrm{MR}$ spectroscopic data were fitted in the time domain. Manual phase correction was performed and subsequently all spectra with water suppression and without were averaged, resulting in one water-suppressed lipid spectrum originating from 128 averages and a non-suppressed spectrum from 24 averages for quantification of the water resonance. Myocardial lipid peaks were fitted by using the Advanced Magnetic Resonance (AMARES) fitting algorithm ${ }^{25}$ within the jMRUI software ${ }^{26}$ as reported earlier ${ }^{13}$. Cardiac lipid content is given as the percentage of the $\mathrm{CH}_{2}$ peak compared to the water resonance, uncorrected for $\mathrm{T} 1$ and $\mathrm{T} 2$ relaxation.

\section{${ }^{31} \mathrm{P}$ MRS}

Single voxel cardiac ${ }^{31} \mathrm{P}-\mathrm{MRS}$ spectra were obtained from the left ventricle with subjects in the supine position as reported previously ${ }^{27}$. A $10 \mathrm{~cm}$ coil was fixed on the chest, at the level of the myocardium. Manual tuning and matching of the ${ }^{31} \mathrm{P}$-surface coil was performed to adjust for different coil loadings. ECG-triggered ISIS was used for localization to the left ventricle ( $T R=3.6 \mathrm{~s}, \mathrm{n}=192)$. Spectroscopy and shimming volumes were planned on the transverse and sagittal scout images to include the entire left ventricle, while avoiding chest wall muscle and diaphragm muscle. A rest slab was placed over the chest muscle to minimize contamination from skeletal muscle.

\section{${ }^{31} \mathrm{P}$ MRS spectral post processing}

${ }^{31} \mathrm{P}-\mathrm{MRS}$ was quantified with the dedicated software jMRUI. Signals were quantified in the time domain. Six Gaussian peaks were fitted using prior knowledge defining chemical shift. The ATP level in the ${ }^{31}$ P-MRS was corrected for the ATP contribution from blood and $T_{1}$ corrected $^{28}$.

\section{Statistics}

Data are presented as mean \pm SE. A two-way ANOVA for repeated measures was performed to analyse data over time for cardiac lipid content, substrate oxidation and plasma values of FA, glucose, triglycerides and glycerol. Statistical differences in ejection fraction and $\mathrm{PCr} /$ 
ATP between the two conditions were determined by a paired student t-test. All statistics were performed using SPSS 16.0 for Mac and $\mathrm{P}<0.05$ was considered statistically significant. 


\section{Results}

\section{Energy expenditure and substrate oxidation}

No significant differences in energy expenditure between the glucose-fed and the fasted state were found neither during exercise nor post exercise (see table 2). During exercise, there was a significant time $(P<0.01)$ and treatment $(p<0.01)$ effect for respiratory quotient (RQ), without a time*treatment interaction ( $p=0.39$ ) effect. RQ was significantly higher at all time points in the glucose-fed state compared with the fasted state $(p<0.01)$ (see figure $2 a)$, reflecting higher carbohydrate oxidation in glucose-fed state, and RQ decreased between $t=30$ and $t=90$ min $(p<0.05)$ in both conditions. In the post exercise period, a significant treatment effect $(p<0.01)$ was revealed, but no time $(p=0.88)$ or time*treatment $(p=0.10)$ effects were present. Also under resting conditions post exercise, RQ was significantly higher in the glucose-fed state compared with the fasted state at all time points $(<0.01)$.

Table 2. Energy expenditure and fat and carbohydrate oxidation during and after exercise in glucose-supplemented and fasted state.

\begin{tabular}{|c|c|c|c|c|c|c|c|}
\hline \multirow[b]{2}{*}{ Treatment } & \multirow[b]{2}{*}{$\begin{array}{l}\text { Time, } \\
\text { min }\end{array}$} & \multicolumn{3}{|c|}{ Glucose } & \multicolumn{3}{|c|}{ Fasted } \\
\hline & & $\mathrm{EE}, \mathrm{kJ} / \mathrm{min}$ & $\begin{array}{c}\text { Fat oxidation, } \\
\mathrm{mg} / \mathrm{min}\end{array}$ & $\begin{array}{c}\mathrm{CHO} \text { oxidation, } \\
\mathrm{mg} / \mathrm{min}\end{array}$ & $\mathrm{EE}, \mathrm{kJ} / \mathrm{min}$ & $\begin{array}{c}\text { Fat oxidation, } \\
\mathrm{mg} / \mathrm{min}\end{array}$ & $\begin{array}{c}\mathrm{CHO} \text { oxidation, } \\
\mathrm{mg} / \mathrm{min}\end{array}$ \\
\hline \multirow[t]{4}{*}{ Exercise } & 30 & $41.9 \pm 1.5$ & $313 \pm 31^{*}$ & $1871 \pm 80^{*}$ & $41.7 \pm 1.6$ & $476 \pm 83$ & $1451 \pm 151$ \\
\hline & 60 & $41.9 \pm 1.2$ & $398 \pm 27 \#$ & $1659 \pm 73 \#$ & $42.0 \pm 1.3$ & $530 \pm 31$ & $1336 \pm 88$ \\
\hline & 90 & $42.3 \pm 1.4$ & $469 \pm 27 \#$ & $1503 \pm 67 \#$ & $42.3 \pm 1.5$ & $635 \pm 16$ & $1092 \pm 85$ \\
\hline & 120 & $43.8 \pm 1.9$ & $467 \pm 3 \#$ & $1606 \pm 137 \#$ & $42.5 \pm 1.8$ & $665 \pm 22$ & $1026 \pm 124$ \\
\hline \multirow{3}{*}{$\begin{array}{c}\text { Post } \\
\text { exercise }\end{array}$} & 240 & $5.3 \pm 0.2$ & $45 \pm 8 \#$ & $226 \pm 19 \#$ & $5.5 \pm 0.2$ & $95 \pm 7$ & $109 \pm 11$ \\
\hline & 300 & $5.4 \pm 0.2$ & $30 \pm 5 \#$ & $267 \pm 17 \#$ & $5.5 \pm 0.2$ & $103 \pm 5$ & $93 \pm 11$ \\
\hline & 360 & $5.4 \pm 0.2$ & $30 \pm 6 \#$ & $269 \pm 18 \#$ & $5.6 \pm 0.2$ & $104 \pm 6$ & $92 \pm 11$ \\
\hline
\end{tabular}

Data are means $\pm \mathrm{SEM}$. EE, energy expenditure; $\mathrm{CHO}$, carbohydrate. $* \mathrm{P}<0.05$ and $\# \mathrm{P}<0.01$ compared with fasted treatment.

During exercise, there was a significant time $(p<0.01)$ and treatment $(p<0.01)$ effect for both carbohydrate and fat oxidation, without a time*treatment interaction $(p=0.38$ and 0.27 , respectively) effect. As depicted in table 2 , carbohydrate oxidation was significantly higher at all time points in the glucose-fed state compared with the fasted state $(p<0.01)$, whereas fat oxidation was significantly higher at all time points in the fasted state compared with the glucose-fed state $(p<0.05)$. Both carbohydrate and fat oxidation increased between $t=30$ and $t=90(p<0.01)$. In the post exercise period the carbohydrate oxidation was significantly higher in the glucose-fed state compared with the fasted state at all time points $(p<0.01)$, whereas 
the fat oxidation was significantly higher in the fasted state compared with the glucose-fed state at all time points $(p<0.01)$. There was no time or time*treatment interaction effect for neither carbohydrate nor fat oxidation in the post exercise period $(P>0.05)$.

\section{Blood plasma concentrations}

During exercise, there was a significant time $(P<0.01)$, treatment $(p<0.01)$ and time*treatment interaction $(p<0.01)$ effect for plasma FA concentrations. Except for baseline $(t=0)$ plasma FA concentrations were higher at all time points during exercise in the fasted state $(p<0.01)$ compared with the glucose-fed state (Fig. 2b). Plasma FA concentrations increased with time during exercise $(p<0.01)$, with this increase being more pronounced in the fasted state compared to the glucose-fed state. In the post exercise period, plasma FA concentrations were higher at all time points in the fasted state compared with the glucose-fed state $(p<0.001)$, but there was no time or time*treatment interaction effect.

During exercise, there was a significant time $(p<0.05)$, treatment $(p<0.01)$ and time*treatment interaction effect $(p<0.01)$ for plasma glucose concentrations. In the fasted state, plasma glucose concentrations decreased during exercise (Fig. 2c), whereas an increase was observed in the glucose-fed state. As a result, plasma glucose levels were higher in the glucose-fed state compared with the fasted state from time point $t=60$ min onwards $(p<0.05)$. In the post exercise period, plasma glucose concentrations were higher at all time points in the glucose-fed state compared with the fasted state $(p<0.01)$, and there was a time*treatment interaction $(p=0.046)$ effect, but there was no time $(p=0.085)$ effect.

During exercise, there was no significant time $(p=0.16)$, treatment $(p=0.22)$ or time*treatment interaction $(p=0.87)$ effect for plasma triglyceride concentrations, whereas there was a tendency to higher plasma triglyceride concentrations post exercise in the glucose-fed state compared with the fasted state $(p=0.069$, fig. $2 d)$. There were no time $(p=0.95)$ or time*treatment interaction $(p=0.99)$ effect for plasma triglyceride concentrations post exercise. Considering all time points of the test day, there is an overall time effect with triglyceride concentrations decreasing over time, being significantly different from baseline levels in the fasted condition from $t=120$ minutes onwards ( $p>0.05)$.

There was a significant time $(p<0.01)$, treatment $(p<0.01)$ and time*treatment interaction $(p<0.01)$ effect for glycerol during exercise. Glycerol concentrations were higher in the fasted state, and increased during exercise, with a more pronounced increased in the fasted state (from $99 \pm 14 \mu \mathrm{mol} / \mathrm{l}$ to $620 \pm 31 \mu \mathrm{mol} / \mathrm{l}$ in the fasted state vs $91 \pm 9 \mu \mathrm{mol} / \mathrm{l}$ to 219 
$\pm 27 \mu \mathrm{mol} / \mathrm{l}$ in the glucose-fed state). Post exercise glycerol was significantly higher in the fasted state compared with the glucose-fed state $(135 \pm 9 \mu \mathrm{mol} / \mathrm{l}$ vs $46 \pm 3 \mu \mathrm{mol} / \mathrm{l}$ at $\mathrm{t}=360$ ) $(p<0.01)$, but there was no time $(0.11)$ or time*treatment interaction $(p=0.22)$ effect.

Figure 2: Changes in Respiratory Quotient and plasma metabolites during the exercise protocol
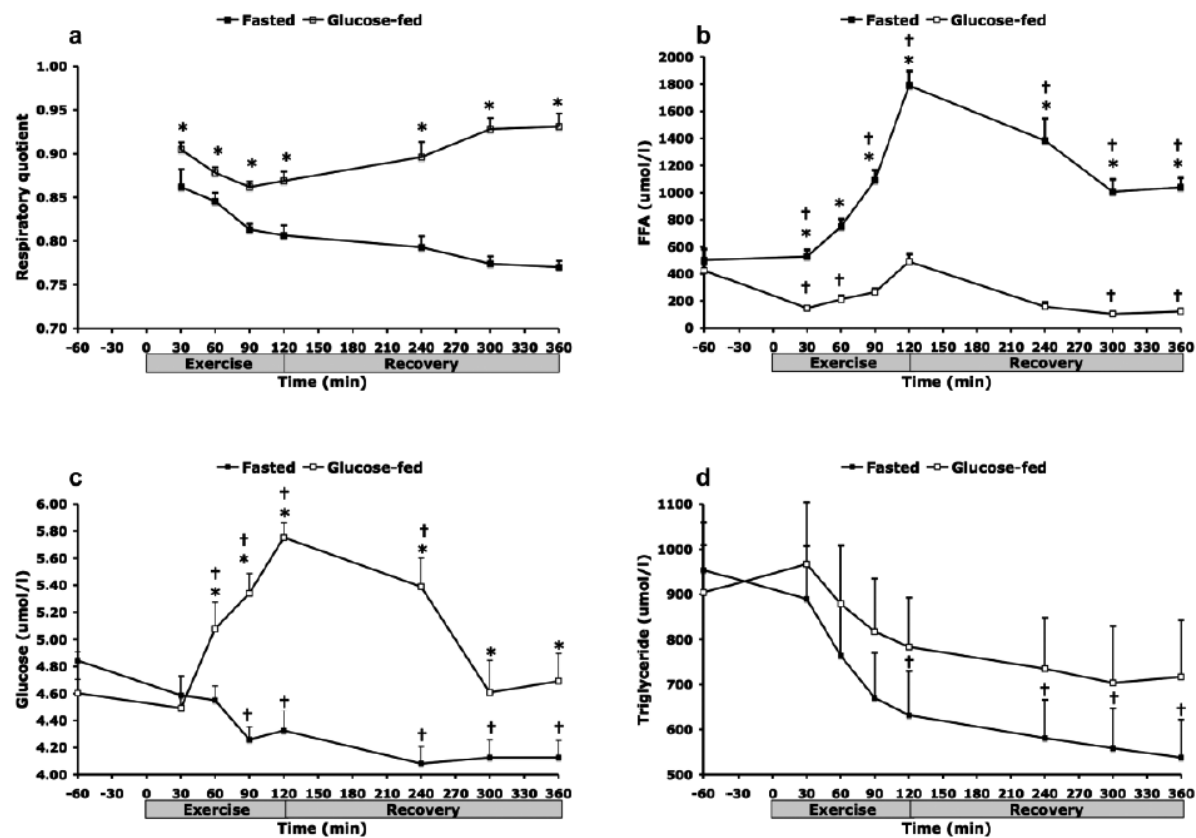

A. Respiratory quotient during and after $2 \mathrm{~h}$ of cycling at $50 \%$ of maximal power output (Wmax) and plasma concentrations of B. (free) fatty acids (FA), C. glucose and D. triglyceride (TG), with (open square) and without (filled square) glucose supplementation. For figure $2 \mathrm{a}, \mathrm{b}, \mathrm{c}$ and $\mathrm{d}$ : $* \mathrm{P}<0.01$ compared with glucose-supplemented condition. For figure $2 b, c$ and $d:+P<0.05$ changes over time compared with baseline $(t=-60)$. Data are mean $\pm S E$.

\section{Cardiac lipid content}

There was a significant treatment $(p=0.029)$ and time*treatment interaction $(p=0.009)$ effect for cardiac lipid content, but no time $(p=0.242)$ effect. As depicted in figure 3 , overall cardiac lipid content was higher at the end of the test day in the fasted state compared with the glucose-fed state, and cardiac lipid content increased after recovery from exercise in the fasted state $(p=0.003)$, whereas it did not change when glucose supplementation was given $(p=0.272)$. At baseline, there were no differences in cardiac lipid content between 
the two conditions $(p=0.239)$. Cardiac lipid content was also not different between the two conditions directly after exercise $(p=0.119)$. However, 4 hours post exercise cardiac lipid content was elevated by $41 \%$ in the fasted state compared with the glucose-fed state $(p=0.008)$.

Figure 3: Cardiac lipid content at baseline, directly post exercise and 4 h post exercise.

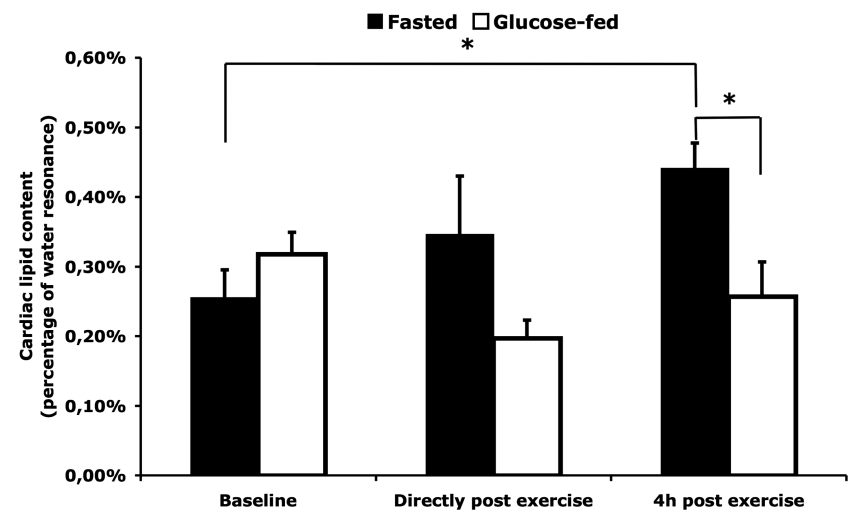

Cardiac lipid content is expressed as the relative intensity of the $\mathrm{CH}_{2}$ peak, compared to the unsuppressed water resonance. $n=9, * P<0.01$. Data are mean $\pm S E$.

\section{Cardiac energy status and function}

In a subgroup of six subjects PCr/ATP was determined four hours post exercise. PCr/ATP was $32 \%$ lower in the fasted state compared with the glucose-fed state ( $p=0.014$, fig. 4$)$. Ejection fraction ( $p=0.038$, fig. 5 ), stroke volume $(115 \pm 6 \mathrm{ml}$ vs $107 \pm 4 \mathrm{ml}, p=0.044)$, cardiac output $(7.2 \pm 0.5 \mathrm{l} / \mathrm{min}$ vs $6.0 \pm 0.2 \mathrm{l} / \mathrm{min}, \mathrm{p}=0.016)$ and cardiac index $\left(3.9 \pm 0.3 \mathrm{l} / \mathrm{min} / \mathrm{m}^{2}\right.$ vs $3.3 \pm 0.2 \mathrm{l} / \mathrm{min} / \mathrm{m}^{2}, \mathrm{p}=0.016$ ) were all significantly higher at $4 \mathrm{~h}$ of recovery in the fasted state compared with the glucose-fed state. At $4 \mathrm{~h}$ of recovery, end diastolic (182 $\pm 8 \mathrm{ml}$ in the fasted state vs $180 \pm 8 \mathrm{ml}$ in the glucose-fed state, $p=0.626$ ) and end systolic ( $68 \pm 4 \mathrm{ml}$ in the fasted state vs $73 \pm 5 \mathrm{ml}$ in the glucose-fed state, $p=0.179$ ) volumes were not significantly different between the fasted and glucose-fed state. A tendency to a higher heart rate during these measurements in the fasted state was found, compared with the glucose-fed state, however it did not reach statistical significance ( $63 \pm 3 \mathrm{bpm}$ vs $58 \pm 3 \mathrm{bpm}, \mathrm{p}=0.097$ ). Interobserver variability of ejection fraction analysis was good with a coefficient of variation of $3.6 \pm 0.9 \%$. 
To compare the values for cardiac function at $4 \mathrm{~h}$ of recovery to baseline reference values without prior exercise, we measured in the same subjects cardiac function after an overnight fast, on a separate day. 4h-recovery values for ejection fraction ( $p=0.026$, fig. 5), cardiac output $(7.2 \pm 0.5 \mathrm{ml}$ vs $5.8 \pm 0.3 \mathrm{ml}, \mathrm{n}=9, \mathrm{p}=0.015)$ and cardiac index $\left(3.7 \pm 0.3 \mathrm{l} / \mathrm{min} / \mathrm{m}^{2}\right.$ vs $3.0 \pm 0.2 \mathrm{l} / \mathrm{min} / \mathrm{m}^{2}, \mathrm{n}=9, \mathrm{p}=0.007$ ) were all significantly higher in the fasted state compared with the reference measurement, whereas the glucose-fed state did not differ from the reference measurement ( $p>0.05)$. There was no difference in stroke volume between the reference measurement and the fasted $(p=0.933)$ or the glucose-fed $(p=0.241)$ states. Heart rate was significantly lower during the reference measurement compared with both the measurement in the fasted ( $52 \pm 4 \mathrm{bpm}$ vs $62 \pm 4 \mathrm{bpm}, \mathrm{n}=9, \mathrm{p}=0.001$ ) and glucose-fed state ( $52 \pm 4 \mathrm{bpm} 58 \pm 3 \mathrm{bpm}, \mathrm{n}=9, \mathrm{p}=0.013$ ) at 4 hours of recovery.

Figure 4: PCr/ATP ratio four hours post exercise

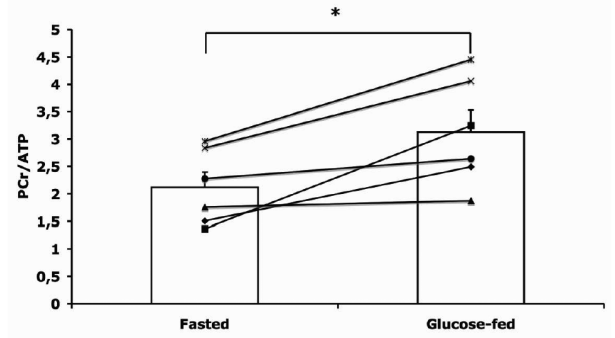

PCr/ATP ratios; $\mathrm{n}=6, * \mathrm{P}=0.014$. Data are depicted both as individual and mean $\pm \mathrm{SE}$.
Figure 5: Left ventricular ejection fraction four hours post exercise

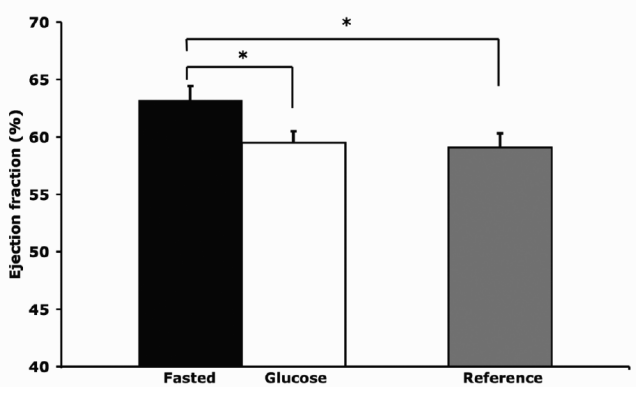

Left Ventricular Ejection fraction, with (white bar) and without (black bar) glucose ingestion, compared with a reference (grey bar) measurement. ${ }^{*} \mathrm{P}<0.05$. Data are mean $\pm \mathrm{SE}$. 


\section{Discussion}

The primary finding of the present study is that cardiac lipid content increases upon exerciseinduced -i.e. physiological - elevation of plasma FA concentrations in healthy lean young men. Furthermore, we revealed that this increase in cardiac lipid content is paralleled by a lower cardiac energy status (lower PCr/ATP), but not by a lowered cardiac function. These results indicate that cardiac muscle is rather unrestricted in its uptake of circulating FA and that the validity of PCr/ATP ratio as a relevant marker of cardiac function can be debated under some circumstances.

There is an increasing prevalence of obesity worldwide and obesity is well known to be an independent risk factor for the development of heart failure. Obesity is associated with excessive storage of lipids (triglycerides) not only in adipose tissue, but also in nonadipose tissues, such as the heart. We show here for the first time that cardiac lipid content is increased upon exercise-induced elevation of plasma FA concentrations in healthy lean young men, and that blunting of the exercise-induced increase in plasma FA concentrations completely prevents the increase in cardiac lipid content. This suggests that in healthy lean young men plasma FA concentration is an important determinant of cardiac lipid content, which in turn suggests that in this population uptake of FA in the heart largely depends on FA availability. This is in agreement with previous studies pointing at the importance of high FA concentrations as determinants of cardiac lipid content ${ }^{14}$. Increased cardiac lipid content is shown to be associated with obesity and diabetes ${ }^{13,14,29,30}$, conditions in which it is well known that plasma FA concentrations are high. Furthermore, fasting for more than 48 hours ${ }^{16}$ or a very low calorie diet for more than three days ${ }^{15}$, which both are characterised by elevated plasma FA concentrations, also resulted in increased cardiac lipid content. Although our results may suggest that elevated plasma FA concentrations may also underlie cardiac lipid accumulation and cardiac dysfunction in obesity and diabetes, it is important to note that major differences exist between obesity,insulin resistance and fasting. Thus, although both conditions are characterized by elevated plasma FA concentrations, plasma leptin and insulin levels are elevated in obesity, but markedly reduced upon fasting. It has recently been shown that under conditions of systemic insulin resistance the heart remains insulin sensitive $^{31}$, therefore chronic stimulation of multiple signaling processes in the myocardium by leptin and insulin may result in cardiac pathology.

Interestingly, our finding that elevated plasma FA lead to cardiac lipid accumulation are to some extent in accordance with observations in skeletal muscle. Thus, we have previously shown that during exercise, when peripheral lipolysis is stimulated to increase plasma FA availability for subsequent oxidation, a net accumulation of intramyocellular lipids occurred 
in non-exercising muscles of the upper arm, indicating that high circulatory FA concentrations also lead to unrestricted uptake of FA in skeletal muscle ${ }^{20}$. However, intramyocellular lipid content was reduced in the exercising muscles of the leg, suggesting that in this active muscle FA delivery was customized to FA oxidation. In that respect, it is surprising that in cardiac muscle, which heavily relies on FA for oxidation during exercise, the elevation of plasma FA lead to an increase rather than a decrease in cardiac lipid content. This suggests that the uptake of FA in cardiac muscle is less well tuned to the oxidative needs compared to skeletal muscle and that even under conditions of increased cardiac work, FA entering the heart are shuttled towards storage rather than to oxidation.

In parallel to the increased cardiac lipid content we also observed a lower cardiac energy status (lower PCr/ATP). Cardiac energy status has a strong prognostic value in heart failure patients and it has been suggested to be an early marker of disturbed cardiac metabolism ${ }^{18}$. In line with this, Scheuermann-Freestone et al. ${ }^{17}$ found that the PCr/ATP ratios correlate negatively with plasma FA concentrations. Furthermore, a decreased PCr/ATP ratio has been found to be associated with diastolic dysfunction and diabetes ${ }^{32}$. As an explanation for this finding, it has been suggested that plasma FA increase the expression of mitochondrial uncoupling proteins in the heart, and these proteins can reduce the efficiency with which mitochondria can produce ATP, thereby resulting in altered PCr/ATP ratios ${ }^{33}$. Some care should be taken when interpreting PCr/ATP ratios as energy status, as the assumption that a decreased $\mathrm{PCr} / \mathrm{ATP}$ ratio is reflecting a decreased $\mathrm{PCr} / \mathrm{Cr}$ ratio can theoretically be biased by a decrease in total creatine concentration. In that context, other studies have shown that indeed creatine concentration in the failing heart is decreased ${ }^{34-36}$. In the current study, we cannot exclude that creatine depletion contributes to the lower PCr/ATP ratio found in the fasted condition.

However, even though an increased cardiac lipid content was associated with a lower cardiac energy status in the present study, the systolic cardiac function was not negatively affected. The systolic cardiac function was determined by multi slice CINE-MRI, which is the gold standard for measuring systolic cardiac function. Together with myocardial spectroscopy this permits examinations of associations between myocardial triglyceride concentrations and cardiac function. Surprisingly, cardiac function was higher in the condition with high plasma FA concentrations and elevated cardiac lipid content compared with the glucose-fed state. Moreover, cardiac function in the fasted state was in fact elevated when compared with the reference condition. Increased concentrations of adrenaline and noradrenaline may be underlying the stronger contraction in that case. Alternatively, changes in peripheral resistance may occur with the current protocol, which may affect ejection fraction. Although the exact mechanism explaining the increased cardiac function in the high plasma FFA 
condition remains speculative, we can conclude that, if anything, the elevated plasma FA concentrations and concomitant cardiac lipid content did not negatively impact cardiac function. This shows that at least in this population PCr/ATP ratio is not a good marker of cardiac function under acute conditions of elevated FA.

In summary, in this study we revealed that elevated plasma FA concentrations, induced by prolonged exercise in the fasted state, increased storage of triglycerides in cardiac tissue but did not acutely hamper systolic function. Although the lower cardiac energy status is in line with a lipotoxic action of cardiac lipids, a causal relationship cannot be proven, and it cannot be excluded that acute prolonged exercise influences cardiac energy status and lipid content independently.

\section{Acknowledgements}

Dr. M. Schär kindly provided the navigator software used for the ${ }^{1} \mathrm{H}-\mathrm{MRS}$ measurements.

Dr. P.Schrauwen is supported by a ' $\mathrm{VICl}$ ' Research Grant for innovative research from the Netherlands Organization for Scientific Research (Grant 918.96.618). This research was supported by the Center for Translational Molecular Medicine and the Netherlands Heart Foundation, Dutch Diabetes Research Foundation and Dutch Kidney Foundation (PREDICCt). 


\section{References}

1. Roger VL, Weston SA, Redfield MM, Hellermann-Homan JP, Killian J, Yawn BP, Jacobsen SJ. Trends in heart failure incidence and survival in a community-based population. Jama. 2004;292:344-350

2. Mokdad AH, Ford ES, Bowman BA, Dietz WH, Vinicor F, Bales VS, Marks JS. Prevalence of obesity, diabetes, and obesity-related health risk factors, 2001. Jama. 2003;289:76-79

3. Kenchaiah S, Evans JC, Levy D, Wilson PW, Benjamin EJ, Larson MG, Kannel WB, Vasan RS. Obesity and the risk of heart failure. N Engl J Med. 2002;347:305-313

4. Murphy NF, MacIntyre K, Stewart S, Hart CL, Hole D, McMurray JJ. Long-term cardiovascular consequences of obesity: 20-year follow-up of more than 15000 middle-aged men and women (the renfrew-paisley study). Eur Heart J. 2006;27:96-106

5. Unger RH, Orci L. Diseases of liporegulation: New perspective on obesity and related disorders. Faseb J. 2001;15:312-321

6. Zhou YT, Grayburn P, Karim A, Shimabukuro M, Higa M, Baetens D, Orci L, Unger RH. Lipotoxic heart disease in obese rats: Implications for human obesity. Proceedings of the National Academy of Sciences of the United States of America. 2000;97:1784-1789

7. Yagyu H, Chen G, Yokoyama M, Hirata K, Augustus A, Kako Y, Seo T, Hu Y, Lutz EP, Merkel M, Bensadoun A, Homma S, Goldberg IJ. Lipoprotein lipase (Ipl) on the surface of cardiomyocytes increases lipid uptake and produces a cardiomyopathy. J Clin Invest. 2003;111:419-426

8. Chiu HC, Kovacs A, Blanton RM, Han X, Courtois M, Weinheimer CJ, Yamada KA, Brunet S, Xu H, Nerbonne JM, Welch MJ, Fettig NM, Sharp TL, Sambandam N, Olson KM, Ory DS, Schaffer JE. Transgenic expression of fatty acid transport protein 1 in the heart causes lipotoxic cardiomyopathy. Circ Res. 2005;96:225-233

9. Chiu HC, Kovacs A, Ford DA, Hsu FF, Garcia R, Herrero P, Saffitz JE, Schaffer JE. A novel mouse model of lipotoxic cardiomyopathy. J Clin Invest. 2001;107:813-822

10. Cheng L, Ding G, Qin Q, Huang Y, Lewis W, He N, Evans RM, Schneider MD, Brako FA, Xiao Y, Chen YE, Yang Q. Cardiomyocyte-restricted peroxisome proliferator-activated receptor-delta deletion perturbs myocardial fatty acid oxidation and leads to cardiomyopathy. Nat Med. 2004;10:1245-1250

11. Nielsen LB, Bartels ED, Bollano E. Overexpression of apolipoprotein $b$ in the heart impedes cardiac triglyceride accumulation and development of cardiac dysfunction in diabetic mice. J Biol Chem. 2002;277:27014-27020

12. Szczepaniak LS, Babcock EE, Schick F, Dobbins RL, Garg A, Burns DK, McGarry JD, Stein DT. Measurement of intracellular triglyceride stores by $\mathrm{h}$ spectroscopy: Validation in vivo. Am J Physiol. 1999;276:E977-989

13. van der Meer RW, Doornbos J, Kozerke S, Schar M, Bax JJ, Hammer S, Smit JW, Romijn JA, Diamant M, Rijzewijk LJ, de Roos A, Lamb HJ. Metabolic imaging of myocardial triglyceride content: Reproducibility of $1 \mathrm{~h} \mathrm{mr}$ spectroscopy with respiratory navigator gating in volunteers. Radiology. 2007;245:251-257

14. Kankaanpaa M, Lehto HR, Parkka JP, Komu M, Viljanen A, Ferrannini E, Knuuti J, Nuutila P, Parkkola R, lozzo P. Myocardial triglyceride content and epicardial fat mass in human obesity: Relationship to left ventricular function and serum free fatty acid levels. J Clin Endocrinol Metab. 2006;91:4689-4695 
15. van der Meer RW, Hammer S, Smit JW, Frolich M, Bax JJ, Diamant M, Rijzewijk LJ, de Roos A, Romijn JA, Lamb HJ. Short-term caloric restriction induces accumulation of myocardial triglycerides and decreases left ventricular diastolic function in healthy subjects. Diabetes. 2007;56:2849-2853

16. Reingold JS, McGavock JM, Kaka S, Tillery T, Victor RG, Szczepaniak LS. Determination of triglyceride in the human myocardium by magnetic resonance spectroscopy: Reproducibility and sensitivity of the method. Am J Physiol Endocrinol Metab. 2005;289:E935-939

17. Scheuermann-Freestone M, Madsen PL, Manners D, Blamire AM, Buckingham RE, Styles P, Radda GK, Neubauer S, Clarke K. Abnormal cardiac and skeletal muscle energy metabolism in patients with type 2 diabetes. Circulation. 2003;107:3040-3046

18. Neubauer S. The failing heart--an engine out of fuel. N EnglJ Med. 2007;356:1140-1151

19. Schrauwen P, Hesselink MK, Vaartjes I, Kornips E, Saris WH, Giacobino JP, Russell A. Effect of acute exercise on uncoupling protein 3 is a fat metabolism-mediated effect. Am J Physiol Endocrinol Metab. 2002;282:E11-17

20. Schrauwen-Hinderling VB, van Loon LJ, Koopman R, Nicolay K, Saris WH, Kooi ME. Intramyocellular lipid content is increased after exercise in nonexercising human skeletal muscle. J Appl Physiol. 2003;95:2328-2332

21. Schrauwen-Hinderling VB, Hesselink MK, Meex R, van der Made S, Schar M, Lamb H, Wildberger JE, Glatz J, Snoep G, Kooi ME, Schrauwen P. Improved ejection fraction after exercise training in obesity is accompanied by reduced cardiac lipid content. J Clin Endocrinol Metab. 2010;95:1932-1938

22. Kuipers H, Verstappen FT, Keizer HA, Geurten P, van Kranenburg G. Variability of aerobic performance in the laboratory and its physiologic correlates. Int J Sports Med. 1985;6:197201

23. Siri WE. The gross composition of the body. Adv Biol Med Phys. 1956;4:239-280

24. Kozerke S, Schar M, Lamb HJ, Boesiger P. Volume tracking cardiac 31p spectroscopy. Magn Reson Med. 2002;48:380-384

25. Vanhamme L, van den Boogaart A, Van Huffel S. Improved method for accurate and efficient quantification of mrs data with use of prior knowledge. J Magn Reson. 1997;129:35-43

26. Naressi A, Couturier C, Devos JM, Janssen M, Mangeat C, de Beer R, Graveron-Demilly D. Java-based graphical user interface for the mrui quantitation package. Magma. 2001;12:141152

27. Lamb HJ, Doornbos J, den Hollander JA, Luyten PR, Beyerbacht HP, van der Wall EE, de Roos A. Reproducibility of human cardiac 31p-nmr spectroscopy. NMR Biomed. 1996;9:217-227

28. Hansch A, Rzanny R, Heyne JP, Leder U, Reichenbach JR, Kaiser WA. Noninvasive measurements of cardiac high-energy phosphate metabolites in dilated cardiomyopathy by using 31p spectroscopic chemical shift imaging. European radiology. 2005;15:319-323

29. Sharma S, Adrogue JV, Golfman L, Uray I, Lemm J, Youker K, Noon GP, Frazier OH, Taegtmeyer $\mathrm{H}$. Intramyocardial lipid accumulation in the failing human heart resembles the lipotoxic rat heart. Faseb J. 2004;18:1692-1700

30. Szczepaniak LS, Dobbins RL, Metzger GJ, Sartoni-D’Ambrosia G, Arbique D, Vongpatanasin W, Unger R, Victor RG. Myocardial triglycerides and systolic function in humans: In vivo evaluation by localized proton spectroscopy and cardiac imaging. Magn Reson Med. 2003;49:417-423 
31. Schwarzer M, Britton SL, Koch LG, Wisloff U, Doenst T. Low intrinsic aerobic exercise capacity and systemic insulin resistance are not associated with changes in myocardial substrate oxidation or insulin sensitivity. Basic Res Cardiol. 2010;105:357-364

32. Rijzewijk LJ, Jonker JT, van der Meer RW, Lubberink M, de Jong HW, Romijn JA, Bax JJ, de Roos A, Heine RJ, Twisk JW, Windhorst AD, Lammertsma AA, Smit JW, Diamant M, Lamb HJ. Effects of hepatic triglyceride content on myocardial metabolism in type 2 diabetes. J Am Coll Cardiol. 2010;56:225-233

33. Murray AJ, Anderson RE, Watson GC, Radda GK, Clarke K. Uncoupling proteins in human heart. Lancet. 2004;364:1786-1788

34. Shen W, Asai K, Uechi M, Mathier MA, Shannon RP, Vatner SF, Ingwall JS. Progressive loss of myocardial atp due to a loss of total purines during the development of heart failure in dogs: A compensatory role for the parallel loss of creatine. Circulation. 1999;100:2113-2118

35. Nakae I, Mitsunami K, Omura T, Yabe T, Tsutamoto T, Matsuo S, Takahashi M, Morikawa S, Inubushi T, Nakamura Y, Kinoshita M, Horie M. Proton magnetic resonance spectroscopy can detect creatine depletion associated with the progression of heart failure in cardiomyopathy. J Am Coll Cardiol. 2003;42:1587-1593

36. Nakae I, Mitsunami K, Yoshino T, Omura T, Tsutamoto T, Matsumoto T, Morikawa S, Inubushi T, Horie M. Clinical features of myocardial triglyceride in different types of cardiomyopathy assessed by proton magnetic resonance spectroscopy: Comparison with myocardial creatine. J Card Fail. 2010;16:812-822 



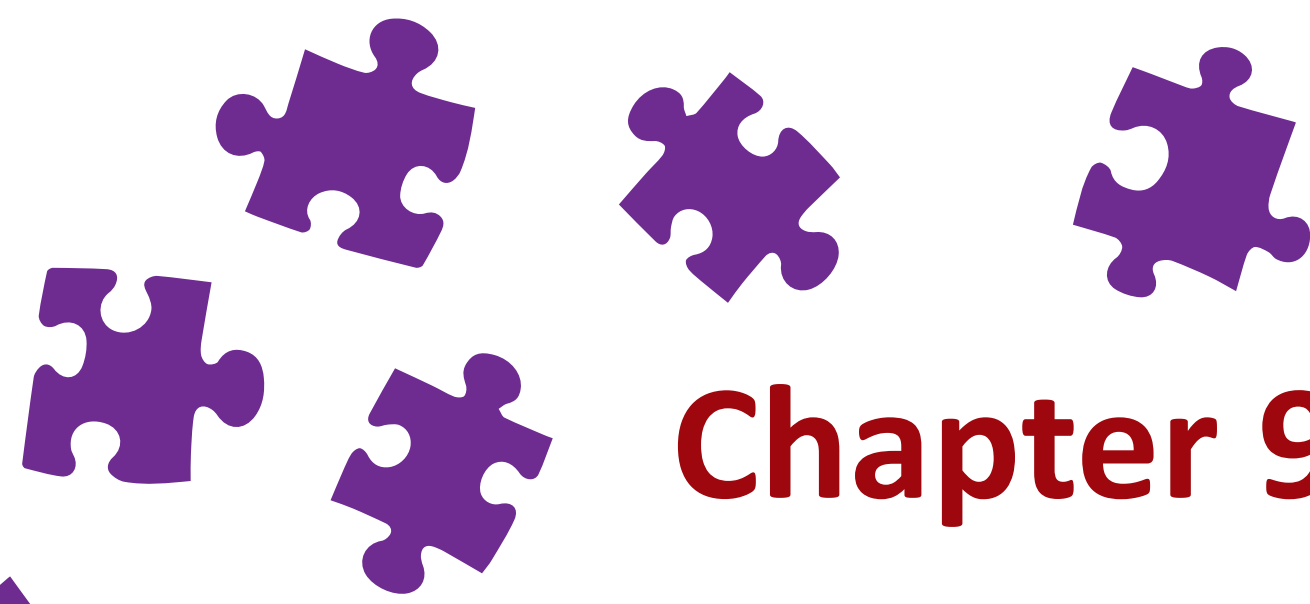

Chapter 9

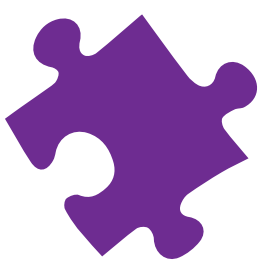

\section{Treatment with Acipimox fails to improve insulin sensitivity and may impair cardiac} function in non-insulin dependent type 2 diabetes mellitus

Tineke van de Weijer ${ }^{1,5 *}$, Esther Phielix ${ }^{2 *}$, Lena Bilet ${ }^{1,5 *}$, Alessandra Laufs ${ }^{2}$, Roshan Livingstone ${ }^{2}$, Peter Nowotny ${ }^{2}$, Lauren M. Sparks ${ }^{1,5}$, Sabina Paglialunga ${ }^{1,5}$, Julia Szendroedi2, ${ }^{2,6}$, Jong-Hee Hwang ${ }^{2}$, Vera B. Schrauwen-Hinderling ${ }^{3,5}$, Matthijs K.C. Hesselink ${ }^{4,5}$, Michael Roden ${ }^{2,6 \#}$, Patrick Schrauwen ${ }^{1,5 \#}$

*,\# these authors contributed equally to this study

${ }^{1}$ Department of Human Biology, Maastricht University Medical Center, The Netherlands, ${ }^{2}$ Institute for Clinical Diabetology, German Diabetes Center, Düsseldorf, Germany, ${ }^{3}$ Department of Radiology, Maastricht University Medical Center, The Netherlands, ${ }^{4}$ Department of Human Movement Sciences, Maastricht University Medical Center, The Netherlands, ${ }^{5}$ School for Nutrition, Toxicology and Metabolism, Maastricht University Medical Center,

The Netherlands, ${ }^{6}$ Departments of Endocrinology and Diabetology and Metabolic Diseases, University Clinics

Düsseldorf, Germany.

(Submitted) 


\section{Abstract}

Background/aim: We tested the hypothesis that treatment with the nicotinic acid derivative Acipimox results in a persistent reduction in free fatty acid (FFA) concentrations, which would improve cardiac function and insulin sensitivity via a reduction in myocardial and skeletal muscle fat accumulation.

Methods: in a multi-center randomized cross-over trial 21 patients with type 2 diabetes (age $57.7 \pm 1.1$ years, body mass index, BMI, $33.4 \pm 0.8 \mathrm{~kg} / \mathrm{m}^{2}$ ) were included. Patients received daily 3 times either $250 \mathrm{mg}$ placebo or Acipimox, each for 2 weeks. We also included 10 age- and BMI-matched non-diabetic subjects $\left(56.0 \pm 2.7\right.$ years, $\left.31.4 \pm 0.8 \mathrm{~kg} / \mathrm{m}^{2}\right)$ who did not receive treatment, as a reference group. Insulin sensitivity was measured with a hyperinsulinemic-euglycemic clamp and a muscle biopsy was taken to assess ex vivo muscle mitochondrial function and lipid accumulation. In a subgroup of participants magnetic resonance spectroscopy was used to measure cardiac fat content $(n=10)$ and cardiac energy status ( $n=10)$, whereas liver fat content and in vivo mitochondrial function was measured in all subjects. Cardiac function was measured with echocardiography $(n=10)$.

Results: Unexpectedly, after 2-weeks of Acipimox treatment, plasma FFA almost doubled (759 \pm 44 vs. $1135 \pm 97 \mu \mathrm{mol} / \mathrm{L}, \mathrm{p}<0.01$ for placebo vs. Acipimox). This was paralleled by an increase in skeletal muscle lipid content and a decrease in in vivo mitochondrial function and insulin sensitivity. Hepatic lipid content remained unchanged, whereas ex vivo mitochondrial function tended to increase. Surprisingly, despite unaltered cardiac fat content and energy status, cardiac function tended to decrease (Ejection Fraction (EF) $59.9 \pm 2.7$ vs. $56.6 \pm 2.0 \%$, $\mathrm{p}=0.08$ for placebo vs. Acipimox).

Conclusions: Treating type 2 diabetic patients with Acipimox leads to a rebound rise in plasma FFA, which further deteriorates insulin sensitivity and cardiac function. The decrease in insulin sensitivity is likely to be mediated through enhanced lipid accumulation and impaired in vivo mitochondrial function in skeletal muscle. However, Acipimox did seem to upregulate mitochondrial function ex vivo, indicating that in the absence of elevated plasma FFA concentrations, Acipimox might have a direct positive effect on the mitochondria. Still, treating hyperlipidemia in insulin resistant patients with nicotinic acid derivatives should be done with care, and further research is needed to assess the safety and efficacy of this drug class. 


\section{Introduction}

The risk of cardiovascular diseases is markedly elevated in patients with type 2 diabetes (T2D), leading to an increased morbidity and mortality rate in T2D patients compared to the general population ${ }^{1}$. Prevention and treatment of the complications of diabetes mellitus have the potential to improve quality of life and life expectancy ${ }^{2,3}$. Hence, early diagnosis and treatment of hyperlipidemia in T2D is important to reduce morbidity and mortality rates in T2D patients. First choice for treatment of hyperlipidemia in T2D is treatment with statins, as these are known for their potent effect on lowering of cholesterol concentrations. However, statins only have a minor effect on triglycerides. Here, nicotinic acid analogues are far more potent, as these drugs form a class of lipid lowering drugs, which inhibit adenylylcyclase, resulting in lowering of hormone sensitive lipase (HSL) in adipose tissue ${ }^{4,5}$. Lowering of HSL results in a diminished release of free fatty acids (FFA) from adipose tissue and increases lipoprotein lipase (LPL) activity in adipose tissue, thereby increasing the uptake and breakdown of very-low density lipoproteins (VLDL).

Acipimox is a nicotinic acid analogue frequently used to treat hyperlipidemia ${ }^{6-8}$. Besides its effects on cholesterol and triglycerides, Acipimox has been described to reduce plasma FFA concentrations by 46-57\% ${ }^{9-11}$, which may lower ectopic fat accumulation in T2 $D^{9-11}$. Excessive fat accumulation in skeletal muscle, liver and heart has been linked to the development of T2D and its co-morbidities ${ }^{12}$. A surplus of circulating FFA, as well as increased storage in skeletal muscle, result in the formation of toxic lipids intermediates known to impede with insulin signaling $2,13,14,15$. Moreover, lipid accumulation in skeletal muscle as well as in liver and heart has been associated with the development of non-alcoholic fatty liver disease ${ }^{16}$ and the development of diabetic cardiomyopathy ${ }^{17,18}$. Hence, a better understanding of the underlying mechanisms leading to diseases associated with ectopic fat accumulation in T2D is needed. In that context, a role for mitochondrial oxidative capacity has been suggested. Some studies show that mitochondrial oxidative capacity is impaired in both patients with type 2 diabetes ${ }^{19-22}$ as well as in pre-type 2 diabetic first-degree relatives ${ }^{19,23}$. Therefore, it is tempting to suggest that a reduction in mitochondrial oxidative capacity will contribute to the ectopic accumulation of insulin signalling-desensitizing lipid intermediates promoting insulin resistance. Indeed, increased skeletal muscle lipid accumulation previously has been shown to be associated with a decreased ATP production ${ }^{23,24}$.

However, alternatively it has been suggested that elevated plasma FFA concentrations may also result in a deterioration of mitochondrial function (for review see Schrauwen et al. BBA, 201025). This mechanism has not been confirmed yet, as studies assessing the acute effects of elevated plasma FFA concentrations did not show any effects on ATP 
production ${ }^{26}$. Therefore, a prolonged sustained lowering of circulating FFA concentrations might be beneficial to unravel these mechanisms. We hypothesized that Acipimox, as a lipidlowering agent, would exert a sustained suppression of adipose tissue lipolysis, resulting in a lower accumulation of ectopic fat, improved mitochondrial function and increased insulin sensitivity. As a consequence, we predict that this might improve cardiac function, skeletal muscle mitochondrial function and hepatic steatosis and reduce co-morbidities of type 2 diabetes. The effects of Acipimox on skeletal muscle lipid accumulation and insulin sensitivity have been investigated before, though, these studies investigated only short acute effects (within 1-2 hours) ${ }^{27,28}$. Here, we investigate prolonged sustained effects of Acipimox administration. Noteworthy, studies investigating the prolonged effects of Acipimox ${ }^{9,27,29-31}$, administered the last dose shortly before the metabolic measurement of interest, and results obtained may therefore reflect the acute effect of the last dose. Therefore, in the present study patients with T2D were administered Acipimox 2-3 times upon each meal daily, according to international clinical standards. We were interested in the sustained and not the acute effects of the last dose of Acipimox administration, and therefore administration of Acipimox directly before the test days was omitted. Hence, in the present study we aimed to investigate whether the effects of 2 weeks of chronic reduction of circulating FFA via administration of Acipimox, would lower ectopic fat accumulation and improve the metabolic profile of T2D. 


\section{Materials and Methods}

\section{Study design}

In the present multi-centre, randomised, double-blind, placebo-controlled cross-over trial, 21 patients with T2D and 10 control participants were included (10 T2D patients and 10 control subjects in the Maastricht University Medical Center and 11 T2D patients in the German Diabetes Center in Düsseldorf). T2D patients were randomly assigned to either placebo or Acipimox ( $250 \mathrm{mg} 3 \mathrm{dd}$ ) treatment for 2 weeks. During these 2 weeks of intervention, diabetic patients were asked to stop their oral glucose lowering medication. Patients were provided with a standardised meal the day prior to the clamp and were advised to refrain from physical exercise 3 days before and during the entire study. At the end of the 2-weeks Acipimox or placebo treatment, measurements were performed to assess cardiac and mitochondrial function, ectopic lipid accumulation and insulin sensitivity. Between interventions, a 4-week washout period was maintained. A control group with similar BMI and age served as a reference group, but did not undergo the intervention.

\section{Subjects}

Both male and post-menopausal female humans were included (see table 1). Before inclusion, participants underwent physical examination and anthropometric measurements and completed a medical history questionnaire, including history of cardiovascular, renal and pulmonary disease, cancer and duration of diabetes. Also routine medical laboratory tests including haematology, and a maximal aerobic capacity test with concurrent ECG were performed as previously described ${ }^{32}$. Body composition was determined using hydrostatic weighing in Maastricht according to Siri et al. ${ }^{33}$. In Dusseldorf body composition was measured with a DEXA scan. Control participants had no family history of T2D and had normal fasting plasma glucose concentrations. T2D patients had well-controlled diabetes (HbA1C $7.08 \pm 0.16 \%$ ) and were either on monotherapy with metformin, or on metformin combined with sulfonylurea. Patients were included when diagnosed with T2D for at least one year. None of the participants included followed a weight-loss dietary program and had a stable body weight for the last 6 months. The Maastricht University Medical Ethical Committee (The Netherlands) and the Medical Association North Rhine in Düsseldorf (Germany), approved the study, and written informed consent was obtained from all participants prior to screening. The study was performed according to the principles expressed in the Declaration of Helsinki. 


\section{Hyperinsulinemic-euglycemic clamp}

All participants underwent a 2-step 6-h hyperinsulinemic-euglycemic clamp (10 and $40 \mathrm{mU}$ $\left./ \mathrm{m}^{2} / \mathrm{min}\right)^{34}$. After an overnight fast, participants received a primed-continuous infusion of $\left\{6,6-{ }^{2} \mathrm{H}_{2}\right\}$ glucose $(0.04 \mathrm{mg} / \mathrm{kg} / \mathrm{min})$ to determine rates of endogenous glucose production (EGP) and whole body glucose disposal rates (WGD) as previously described ${ }^{35}$. After 180 minutes, low insulin infusion was started $\left(10 \mathrm{mU} / \mathrm{m}^{2} / \mathrm{min}\right.$ ) with co-infusion of $0.1 \mu \mathrm{g} / \mathrm{kg} / \mathrm{min}$ of somatostatin ${ }^{24}$ for 3.5 hours until a steady state was reached, after which blood sampling and indirect calorimetry was performed during 30 minutes. Thereafter, high insulin infusion was started (40 mU/m²/min) with co-infusion of $0.1 \mu \mathrm{g} / \mathrm{kg} / \mathrm{min}$ somatostatin for 1.5 hours, after which steady state was reached and blood sampling and indirect calorimetry were repeated.

\section{Indirect calometry}

During the clamp, oxygen consumption and carbon dioxide production were measured with a daily-calibrated automated respiratory gas analyzer using a ventilated hood system (Omnical, IDEE, Maastricht, The Netherlands; Vmax Encore 29n, SensorMedix, Cardinal Health Germany, Hoechberg, Germany). Whole body glucose and fat oxidation rates were calculated using stoichiometric equations based on measured oxygen consumption and carbon dioxide concentrations ${ }^{36}$ with the assumption that protein oxidation was negligible.

\section{Muscle biopsy}

Muscle biopsies were taken from the $m$. vastus lateralis, prior to the clamp under local anesthesia ( $2 \%$ lidocaine), according to the Bergstrom technique ${ }^{37}$. In the muscle tissue obtained, lipid accumulation was assessed histochemically in cross-sections using a modified oil red $\mathrm{O}$ staining for fluorescence microscopy ${ }^{38}$. In addition, $\sim 30 \mathrm{mg}$ of the muscle tissue was used for high-resolution respirometry to determine ex vivo mitochondrial function.

\section{Permeabilization of muscle fibers}

A small portion of the muscle biopsy sample ( $30 \mathrm{mg})$ was immediately placed in icecold biopsy containing preservation medium (BIOPS; OROBOROS Instruments, Innsbruck, Austria). Muscle fibres were permeabilized with saponin according to the technique of Veksler et al. ${ }^{39}$. After completion of the permeabilization protocol, muscle fibres were transferred into ice-cold mitochondrial respiration buffer (MiRO5; OROBOROS Instruments, Innsbruck, Austria). Subsequently, the muscle fibres were transferred to the oxygraph to 
perform high-resolution respirometry as previously described ${ }^{40}$, and were corrected for wet weight.

\section{High-resolution respirometry}

Mitochondrial function ex vivo was determined by measuring oxygen consumption polarographically using a two-chamber Oxygraph (OROBOROS Instruments). Oxygen consumption, or oxygen flux, reflects the first derivative of the oxygen concentration (nmol/ $\mathrm{ml}$ ) in the respiration chambers, expressed as (pmol/(s*mg)), corrected for wet weight muscle tissue (2-5 mg). To evaluate mitochondrial oxidative capacity, different substrate protocols were applied. In every protocol, $4.0 \mathrm{mmol} / \mathrm{l}$ malate was added to obtain state 2 respiration followed by addition of $8.0 \mathrm{mmol} / \mathrm{I}$ glutamate as a substrate for complex l, which was combined with or without $40.0 \mu \mathrm{mol} / \mathrm{I}$ palmitoyl-carnitine. In addition, an excess of $1.6 \mathrm{mmol} / \mathrm{I}$ ADP was added to evaluate state 3 respiration of complex I (state 3 respiration reflects substrate oxidation coupled to energy production). Then $8.0 \mathrm{mmol} / \mathrm{l}$ succinate was added to obtain state 3 respiration from complex I and II. Finally, titrations (in steps of $0.5 \mu \mathrm{l}$ of $1.0 \mathrm{mmol} / \mathrm{l}$ ) of the chemical uncoupler fluoro-carbonyl cyanide phenylhydrazone (FCCP) were added to evaluate maximal respiratory capacity, state $U$.

\section{Measures of mitochondrial density}

Mitochondrial DNA (mtDNA) copy number was determined as a marker for mitochondrial density using quantitative real-time PCR, based on the TaqMan probe method. mtDNA copy number was calculated from the ratio of $\mathrm{NADH}$ dehydrogenase subunit 1 (ND1) to lipoprotein lipase (LPL) (mtDNA/nuclear DNA), as described previously ${ }^{19}$.

\section{Hepatic lipid content by ${ }^{1} H-M R S$}

${ }^{1} \mathrm{H}$-MRS was used to quantify IHL in all subjects on a 3T whole-body scanner (Achieva, Philips Healthcare, Best, The Netherlands) using a two or five-element coil as previously described ${ }^{41}$ with a repetition time of $4000 \mathrm{~ms}$, echo time of $32.5 \mathrm{~ms}$, and number of averages of 64 . To minimize motion artifacts, participants were asked to breathe in the rhythm of the measurement and to be at end-expiration during the acquisition of the spectra. To determine the intensity of the lipid peak, the water signal was suppressed using frequency-selective pre-pulses. The unsuppressed water resonance was used as internal reference (number of averages $=64)$, and spectra were fitted with AMARES ${ }^{42}$ in the jMRUI software ${ }^{43}$. Values are 
given as T2-corrected ratios (according to Hamilton et al., 201144) of the $\mathrm{CH}_{2}$-peak, relative to the unsuppressed water resonance (as percentage).

\section{Cardiac lipid content by ${ }^{1} \mathrm{H}-\mathrm{MRS}$}

Cardiac lipid content was determined at the Maastricht University Medical center (10 diabetic subjects and 10 controls) only, using in vivo ${ }^{1} \mathrm{H}-\mathrm{MRS}{ }^{45}$. Respiratory gating and tracking was performed with a pencil beam navigator placed on the diaphragm ${ }^{46}$. Chemical shift selective water suppression was performed to acquire spectra of the lipid metabolites using a series of 32 spectra (NSA=2 each), resulting in a total of 64 acquisitions. To acquire a reference spectrum of the unsuppressed water peak in the same volume of interest, the acquisition was repeated, with the water suppression pulse off resonance, using a series of 12 spectra (NSA=2 each), resulting in a total of 24 acquisitions. From 10 diabetic subjects, 1 had to be excluded from the cardiac lipid content analysis due to poor quality of spectra. Post-processing of the spectra was performed with the jMRUI software ${ }^{47}$. Manual phase correction was performed, and subsequently, all spectra with water suppression and without were averaged, resulting in one water-suppressed lipid spectrum originating from 128 averages and a non-suppressed spectrum from 24 averages for quantification of water resonance. Myocardial lipid peaks were fitted in the time domain by using the Advanced magnetic resonance (AMARES) fitting algorithm ${ }^{48}$ within the jMRUI software ${ }^{43}$ as reported ${ }^{46}$. Cardiac lipid content is given as the percentage of the $\mathrm{CH}_{2}$-peak compared to water resonance and without correction for $\mathrm{T} 1$ and $\mathrm{T} 2$ relaxation.

\section{Cardiac energy status by ${ }^{31} \mathrm{P}-\mathrm{MRS}$}

Cardiac energy status was determined in vivo with ${ }^{31} \mathrm{P}-\mathrm{MRS}$, as reported previously ${ }^{49}$, only in the subjects included at the Maastricht University Medical Center. Single voxel cardiac ${ }^{31} \mathrm{P}-\mathrm{MRS}$ spectra were obtained from the left ventricle with subjects in the supine position ${ }^{50}$. A $10-\mathrm{cm}$ coil was fixed on the chest, at the position of the myocardium. Manual tuning and matching of the ${ }^{31} \mathrm{P}$-surface coil was performed to adjust for different coil loadings. ECG-triggered ISIS (Image-selected in vivo spectroscopy) was used for localization to the left ventricle ( $T R=3.6 s, n=192$ ). Spectroscopy and shimming volumes were planned on the transverse and sagittal scout images to include the entire left ventricle, while avoiding chest wall muscle and diaphragm muscle. A rest slab was placed over the chest muscle to minimize contamination from skeletal muscle. From the 10 T2D subjects, 3 subjects had to 
be excluded due to a poor quality of the spectra. Likewise, 2 out of 10 controls subjects had to be excluded as well.

${ }^{31} \mathrm{P}-\mathrm{MRS}$ was quantified in the time domain with the AMARES algorithm ${ }^{48}$ in $\mathrm{jMRUI}$ software. Six Gaussian peaks were fitted using prior knowledge defining chemical shift. The ATP concentration assessed with ${ }^{31} \mathrm{P}-\mathrm{MRS}$ was corrected for the ATP contribution from blood and T1- corrected $^{51}$.

\section{PCr-recovery by ${ }^{31} \mathrm{P}-\mathrm{MRS}$}

PCr-recovery was measured in all participants using 31P-MRS to determine in vivo mitochondrial function as previously described22. The test was performed one day prior to the clamp test. A knee-extension protocol was performed on a custom-built magnetic resonance compatible ergometer with a pulley system in a 1.5 and 3T whole-body MRI scanner (Intera; Philips Medical Systems, Best, the Netherlands). The knee extension exercise was performed for 5 min with weight corresponding to $50-60 \%$ of the subject's predetermined maximal knee-extension capacity. Post-exercise PCr kinetics were computed as previously described 22 .

\section{Plasma assays}

Blood collected in tubes containing EDTA was immediately centrifuged and plasma stored at $-80^{\circ} \mathrm{C}$ until assayed. Plasma free fatty acids (FFAs) and glucose were measured with enzymatic assays on a Cobas Fara/Mira (FFA: Wako Nefa C test kit; Wako Chemicals, Neuss, Germany; Glucose: hexokinase method; Roche, Basel, Switzerland). Insulin concentration was determined using a radioimmunoassay (Linco Reseach, St. Charles, MO). Cholesterol, LDL and triglycerides were measured colorimetrically (Roche, Vienna, Austria).

\section{Gas chromatography-mass spectrometry}

Determination of atom percent enrichement (APE) of ${ }^{2} \mathrm{H}$ was done as previously described (Stingle krssak, krebs, Diabetologia 2001:44:48-54) after deproteinization. Briefly, $100 \mu$ l KFEDTA plasma was diluted with an equal amount of water and deproteinized after adding $300 \mu \mathrm{l}$ of $0.3 \mathrm{~N} \mathrm{ZnSO}_{4}$ solution followed by $300 \mu \mathrm{l}$ of $0.3 \mathrm{~N} \mathrm{Ba}(\mathrm{OH})_{2}$ solution. After vortexing for 20 minutes, samples were centrifugated $(21.000 \mathrm{~g}$, room temperature). Then, $400 \mu \mathrm{l}$ were evaporated under a stream of nitrogen 5.0 at $37^{\circ} \mathrm{C}$ and both endogenous and infused $\left\{6,6-{ }^{2} \mathrm{H}_{2}\right\}$ glucose were dericatized with $\mathrm{HOX}(100 \mu \mathrm{l}$ of $2 \%$ solution in pyridine, 60 min $90^{\circ} \mathrm{C}$, cooling for $5 \mathrm{~min}$ ) and acetic anhydride $\left(200 \mu \mathrm{l}, 60 \mathrm{~min}\right.$ at $90^{\circ} \mathrm{C}$, cooling for $5 \mathrm{~min}$ ) to the aldonitrile-pentaacetate. The analyses were performed on a Hewlet-Packard 6890 
gas chromatograph equipped with a 25-m CPSil5CB capillary column $(0.2 \mathrm{~mm}$ i.d., $0.12 \mu \mathrm{m}$ film thickness; Chrompack/Varian, Middelburg, Netherlands) and interfaced to a Hewlett Packard 5975 mass selective detector. Selected ion monitoring was used to determine enrichments of the fragments C 3 to C 6 with the average mass units 187 for the endogenous glucose and 189 for the $\left\{6,6-{ }^{2} \mathrm{H}_{2}\right\}$ glucose. Intra-and inter assay CVs were $0.6 \%$ and 1.0 .

\section{Calculations}

Steele's single-pool non-steady-state equations were used to calculate WGD and EGP52. Volume of distribution was assumed to be $0.160 \mathrm{l} / \mathrm{kg}$ for glucose. Non-oxidative glucose disposal (NOGD) was calculated as WGD minus carbohydrate oxidation.

\section{Statistics}

Data are reported as means \pm SE. Statistical analyses were performed using SPSS version 16.0.2 for Mac OS X (SPSS Inc., NC, USA). Differences between the interventions were analyzed with a 2-tailed, paired student t-test. Differences between T2D patients and obese control subjects were tested with a 2-tailed, unpaired student t-test. Statistical significance was set a priori at $p<0.05$. 


\section{Results}

\section{Basic characteristics}

An overview of the patient characteristics is given in table 1. Control subjects and T2D patients had similar weight, $\mathrm{BMI}$, fat percentage and $\mathrm{VO}_{2}$ max. By design, plasma glucose was elevated in T2D patients $(5.5 \pm 0.1$ vs. $7.4 \pm 0.2 \mathrm{mmol} / \mathrm{L}, \mathrm{p}<0.01$, for controls and T2D patients). $\gamma$-GT was elevated in the T2D patients compared to controls ( $28.1 \pm 3.3$ vs. $41.6 \pm 3.5$ $U / L, p<0.05$, for controls and T2D patients), though the other liver enzymes were similar and within normal range. Baseline cholesterol and plasma FFA were similar between groups, but plasma triglycerides were elevated in the T2D patients $(1.12 \pm 0.14$ vs. $1.98 \pm 0.21 \mathrm{mmol} / \mathrm{L}$, $p<0.05$, for controls and T2D patients).

Table 1: Basic characteristics

\begin{tabular}{|c|c|c|c|c|c|c|c|}
\hline \multirow{3}{*}{$\frac{\text { male/female }}{\text { Age (years) }}$} & \multicolumn{3}{|c|}{ Controls } & \multicolumn{4}{|c|}{ Type 2 diabetics } \\
\hline & \multicolumn{3}{|c|}{$n=9 / 1$} & \multicolumn{3}{|c|}{$n=18 / 3$} & \\
\hline & 56,0 & \pm & 2,7 & 57,7 & \pm & 1,1 & \\
\hline weight (kg) & 96,0 & \pm & 4,4 & 100,5 & \pm & 2,9 & \\
\hline BMI (kg/m2) & 31,4 & \pm & 0,8 & 33,4 & \pm & 0,8 & \\
\hline Fat mass (\%) & 35,3 & \pm & 2,4 & 35,1 & \pm & 1,2 & \\
\hline $\operatorname{VO} 2 \mathrm{max}(\mathrm{ml} / \mathrm{min} / \mathrm{kg})$ & 27,2 & \pm & 1,5 & 24,0 & \pm & 1,2 & \\
\hline Systolic Bloodpressure (mmHg) & 144,6 & \pm & 3,9 & 148,9 & \pm & 2,6 & \\
\hline Diastolic Bloodpressure (mmHg) & 92,8 & \pm & 1,9 & 92,8 & \pm & 1,9 & \\
\hline Fasting plasma glucose (mmol/L) & 5,5 & \pm & 0,1 & 7,4 & \pm & 0,2 & ** \\
\hline HbA1c (\%) & 5,6 & \pm & 0,1 & 7,1 & \pm & 0,2 & $* *$ \\
\hline ASAT (U/L) & 20,1 & \pm & 1,4 & 23,7 & \pm & 1,7 & \\
\hline ALAT (U/L) & 31,3 & \pm & 3,5 & 35,9 & \pm & 3,2 & \\
\hline y-GT (U/L) & 28,1 & \pm & 3,3 & 41,6 & \pm & 3,5 & * \\
\hline Total Cholesterol (mmol/L) & 5,3 & \pm & 0,4 & 8,4 & \pm & 3,5 & \\
\hline $\mathrm{HDL}(\mathrm{mmol} / \mathrm{L})$ & 1,6 & \pm & 0,5 & 1,2 & \pm & 0,1 & \\
\hline LDL (mmol/L) & 3,3 & \pm & 0,4 & 2,6 & \pm & 0,2 & \\
\hline Triglycerides (mmol/L) & 1,1 & \pm & 0,1 & 2,0 & \pm & 0,2 & * \\
\hline FFA ( $\mu \mathrm{mol} / \mathrm{L})$ & 773 & \pm & 184 & 672 & \pm & 70 & \\
\hline
\end{tabular}




\section{Effect of Acipimox on plasma metabolite concentrations}

In contrast to our expectations, we observed that plasma FFA increased rather than decreased upon Acipimox treatment. Fasting plasma FFA concentrations increased from $0.77 \pm 0.05 \mathrm{mmol} / \mathrm{L}$ in placebo condition, to $1.13 \pm 0.10 \mathrm{mmol} / \mathrm{L}$ in Acipimox treated condition $(p<0.01$, see figure $1 A)$. This observation of elevated FFA concentrations under basic conditions was also observed during the clamp; FFA concentrations remained significantly elevated throughout the insulin-stimulated period of the clamp (figure 1). While plasma FFA were elevated, cholesterol and triglyceride concentrations tended to decrease upon Acipimox treatment, indicating that administration of Acipimox was effective in our patients (Total cholesterol; $5.63 \pm 0.28$ vs. $5.05 \pm 0.28 \mathrm{mmol} / \mathrm{L}, \mathrm{p}=0.08$, and TG; $2.60 \pm 0.41$ vs. $1.68 \pm 0.27$ $\mathrm{mmol} / \mathrm{L}, \mathrm{p}=0.08$, for placebo vs. Acipimox).

Acipimox did not affect plasma glucose concentrations $(9.0 \pm 0.8$ vs. $9.2 \pm 0.5 \mathrm{mmol} / \mathrm{L}, \mathrm{p}=0.90$, for placebo vs. Acipimox). Fasting plasma insulin concentrations increased upon Acipimox treatment ( $21.4 \pm 2.5$ vs. $26.9 \pm 3.3, p<0.05$, for placebo vs. Acipimox).

Figure 1: Plasma FFA concentrations during the clamp

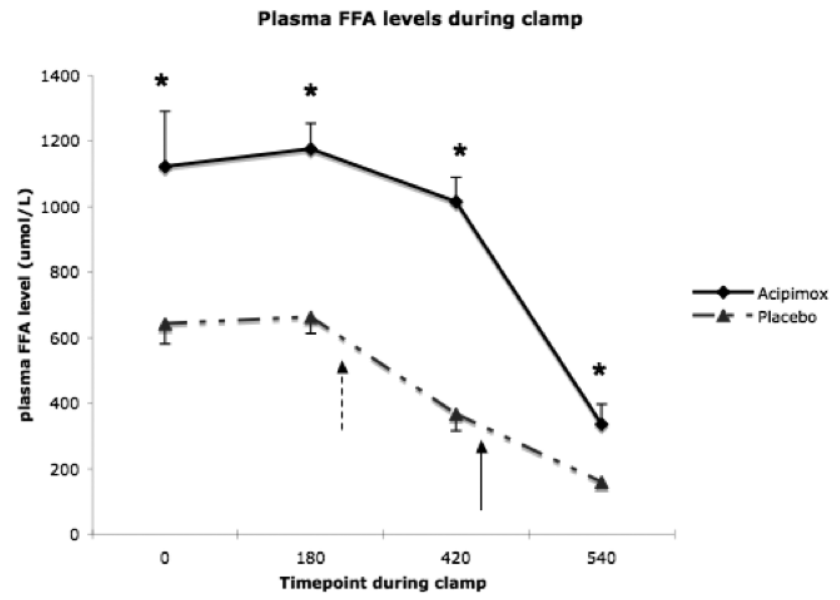

Plasma FFA concentrations in T2D subjects during the clamp. Acipimox treatment resulted in elevated plasma FFA concentrations, and remained significantly elevated throughout the clamp. Indicated with the first dotted arrow is the start of $10 \mathrm{mU} / \mathrm{m} 2 / \mathrm{min}$ infusion of insulin, and indicated with the full arrow is the start of $40 \mathrm{mU} / \mathrm{m} 2 / \mathrm{min}$ infusion of insulin. Significant differences $p<0.05$ are indicated by *. 


\section{Insulin sensitivity}

By design, T2D patients were insulin resistant. Both whole body glucose disposal (WGD) and non-oxidative glucose disposal (NOGD) were higher in T2D and suppression of endogenous glucose production (EGP) was significantly lower in T2D patients compared to controls (see table 2). Furthermore, T2D patients were metabolically less flexible, as the change in RQ upon insulin stimulation was significantly lower (see table 2).

Upon Acipimox treatment, non-insulin stimulated and at low insulin concentrations, there were no significant differences in insulin sensitivity and metabolic flexibility between the Acipimox and placebo treated condition in the T2D patients. However, at high insulin concentrations $\left(40 \mathrm{mU} / \mathrm{m}^{2} / \mathrm{min}\right) \mathrm{WGD}$ and NOGD were lower in the Acipimox treated condition compared to placebo (WGD 20.0 \pm 2.6 vs. $13.5 \pm 2.1, p=0.03$, NOGD $9.9 \pm 2.6$ vs. $2.5 \pm 3.1 \mu \mathrm{mol} / \mathrm{kg} / \mathrm{min}, \mathrm{p}=0.02$, for placebo vs. Acipimox, see figure $2 \mathrm{~A})$. Also EGP was less suppressed by insulin upon Acipimox treatment (EGP $1.7 \pm 0.8$ vs. $3.4 \pm 0.5 \mu \mathrm{mol} / \mathrm{kg} / \mathrm{min}$, $p=0.05$, for placebo vs. Acipimox, see figure $2 B$ ). Neither $R Q$, nor glucose and fat oxidation rates were different upon Acipimox treatment.

\section{Ectopic lipid content}

T2D patients had higher hepatic lipid content compared to control subjects $(3.12 \pm 0.01 \% \mathrm{vs}$. $8.40 \pm 0.01 \%, p=0.03$, in controls vs. T2D patients). Two out of 10 control subjects and 13 out of 21 T2D subjects had a fatty liver. There was no difference in cardiac lipid content between T2D patients and controls (data not shown).

Upon Acipimox treatment, skeletal muscle lipid content significantly increased from $2.8 \pm 0.5 \%$ to $3.8 \pm 0.8 \%$ of total fibre area, $\mathrm{p}<0.05$ (figure $3 \mathrm{~A}$ ). However, Acipimox treatment did not alter hepatic lipid accumulation $(8.40 \pm 0.01 \%$, vs. $8.77 \pm 0.01 \%, p=0.42$, in placebo vs. Acipimox, figure $3 B$ ). In a subgroup of the T2DM patients $(n=10)$, also cardiac lipid content was measured. Here, Acipimox did not have an effect on cardiac lipid accumulation $(0.62 \pm 0.07$ vs. $0.73 \pm 0.12 \% \mathrm{CH}_{2} /$ water resonance, $p=0.38$, for placebo vs. Acipimox, figure $3 \mathrm{C}$ ).

\section{Cardiac function and energy status}

Ejection fraction (EF), Early filling over Atrial filling phase (E/A) ratio and cardiac energy status were not different between T2D subjects and controls. These data indicate that T2D patients in this study had normal cardiac function. Surprisingly, upon Acipimox treatment both systolic and diastolic function tended to decrease in our T2D subjects (EF 59.9 \pm 2.7 vs. $56.5 \pm 2.0 \%, p=0.08$ and $E / A 1.08 \pm 0.12$ vs. $0.94 \pm 0.07, p=0.08$ for placebo vs. Acipimox, figure $4 A$ and $B$ ). However, Acipimox treatment did not affect cardiac energy status (2.20 \pm 0.18 vs. $2.50 \pm 0.27, p=0.52$, in placebo vs. Acipimox, figure 4 C). 
Table 2: Results of the hyperinsulinemic-euglycemic clamp

\begin{tabular}{|c|c|c|c|c|c|c|c|}
\hline \multirow[b]{3}{*}{ Glucose (mmol/L) } & \multirow[b]{3}{*}{ Basal } & \multirow{2}{*}{\multicolumn{3}{|c|}{$\begin{array}{c}\text { Control subjects } \\
(n=10,9 / 1)\end{array}$}} & \multirow{2}{*}{\multicolumn{3}{|c|}{$\begin{array}{c}\text { Type } 2 \text { diabetic patients } \\
(n=21,18 / 3)\end{array}$}} \\
\hline & & & & & & & \\
\hline & & 6,3 & \pm & 0,4 & 9,0 & \pm & 0,8 \\
\hline & $10 \mathrm{mU}$ Insulin & 5,6 & \pm & 0,1 & 6,4 & \pm & 0,3 \\
\hline & 40mU insulin & 5,5 & \pm & 0,1 & 5,3 & \pm & 0,1 \\
\hline \multirow[t]{3}{*}{ NEFA ( $\mu \mathrm{mol} / \mathrm{L})$} & Basal & 692 & \pm & 51 & 759 & \pm & 44 \\
\hline & 10mU Insulin & 278 & \pm & 35 & 401 & \pm & 44 \\
\hline & $40 \mathrm{mU}$ insulin & 133 & \pm & 19 & 179 & \pm & 20 \\
\hline \multirow[t]{3}{*}{ Insulin (mU/L) } & Basal & 22,1 & \pm & 4,5 & 21,4 & \pm & 2,5 \\
\hline & $10 \mathrm{mU}$ Insulin & 28,0 & \pm & 1,2 & 27,1 & \pm & 1,7 \\
\hline & $40 \mathrm{mU}$ insulin & 111,0 & \pm & 5,3 & 120,6 & \pm & 16,7 \\
\hline \multirow[t]{3}{*}{$\mathrm{EE}(\mathrm{KJ} / \mathrm{min})$} & Basal & 1,73 & \pm & 0,11 & 2,05 & \pm & 0,06 \\
\hline & $10 \mathrm{mU}$ Insulin & 1,77 & \pm & 0,13 & 1,97 & \pm & 0,06 \\
\hline & $40 \mathrm{mU}$ insulin & 1,84 & \pm & 0,09 & 1,99 & \pm & 0,07 \\
\hline \multirow[t]{5}{*}{$\mathrm{RQ}$} & Basal & 0,81 & \pm & 0,02 & 0,78 & \pm & 0,01 \\
\hline & $10 \mathrm{mU}$ Insulin & 0,85 & \pm & 0,02 & 0,82 & \pm & 0,01 \\
\hline & Delta B-10mU & 0,03 & \pm & 0,00 & 0,04 & \pm & 0,00 \\
\hline & $40 \mathrm{mU}$ insulin & 0,91 & \pm & 0,04 & 0,85 & \pm & 0,02 \\
\hline & Delta B-40mU & 0,10 & \pm & 0,02 & 0,07 & \pm & 0,01 \\
\hline \multirow{3}{*}{$\begin{array}{l}\text { glucose oxidation } \\
\text { ( } \mu \mathrm{mol} / \mathrm{kg} / \mathrm{min})\end{array}$} & Basal & 6,7 & \pm & 1,2 & 5,0 & \pm & 0,5 \\
\hline & $10 \mathrm{mU}$ Insulin & 8,7 & \pm & 2,0 & 7,3 & \pm & 0,7 \\
\hline & $40 \mathrm{mU}$ insulin & 9,9 & \pm & 2,2 & 9,7 & \pm & 1,4 \\
\hline \multirow{3}{*}{$\begin{array}{l}\text { lipid oxidation ( } \mu \mathrm{mol} / \\
\mathrm{kg} / \mathrm{min} \text { ) }\end{array}$} & Basal & 2,7 & \pm & 0,4 & 3,5 & \pm & 0,2 \\
\hline & $10 \mathrm{mU}$ Insulin & 2,0 & \pm & 0,2 & 2,6 & \pm & 0,2 \\
\hline & $40 \mathrm{mU}$ insulin & 1,2 & \pm & 0,4 & 2,1 & \pm & 0,2 \\
\hline \multirow[t]{5}{*}{$\mathrm{EGP}(\mu \mathrm{mol} / \mathrm{kg} / \mathrm{min})$} & Basal & 6,1 & \pm & 0,8 & 9,8 & \pm & 0,9 \\
\hline & $10 \mathrm{mU}$ Insulin & 2,7 & \pm & 0,9 & 4,9 & \pm & 0,7 \\
\hline & $\%$ suppression & $-59,8$ & \pm & 12,4 & $-44,9$ & \pm & 9,9 \\
\hline & $40 \mathrm{mU}$ insulin & 1,1 & \pm & 1,6 & 1,7 & \pm & 0,8 \\
\hline & $\%$ suppression & $-103,0$ & \pm & 21,9 & $-80,4$ & \pm & 13,1 \\
\hline \multirow[t]{5}{*}{ WGD ( $\mu \mathrm{mol} / \mathrm{kg} / \mathrm{min})$} & Basal & 6,5 & \pm & 0,8 & 10,2 & \pm & 0,9 \\
\hline & $10 \mathrm{mU}$ Insulin & 11,1 & \pm & 1,4 & 7,6 & \pm & 0,7 \\
\hline & $\%$ stimulation & 4,6 & \pm & 1,7 & $-2,6$ & \pm & 1,1 \\
\hline & $40 \mathrm{mU}$ insulin & 31,9 & \pm & 5,0 & 20,0 & \pm & 2,6 \\
\hline & $\%$ stimulation & 25,4 & \pm & 5,1 & 9,7 & \pm & 2,7 \\
\hline \multirow[t]{5}{*}{ NOGD ( $\mu \mathrm{mol} / \mathrm{kg} / \mathrm{min})$} & Basal & 0,1 & \pm & 0,8 & 4,8 & \pm & 1,0 \\
\hline & $10 \mathrm{mU}$ Insulin & 2,6 & \pm & 1,5 & 0,1 & \pm & 0,7 \\
\hline & $\%$ stimulation & 2,5 & \pm & 0,6 & $-4,7$ & \pm & 1,2 \\
\hline & $40 \mathrm{mU}$ insulin & 14,3 & \pm & 3,5 & 9,9 & \pm & 2,6 \\
\hline & $\%$ stimulation & 14,2 & \pm & 2,6 & 5,1 & \pm & 3,0 \\
\hline
\end{tabular}

$\#=p<0.10$ between T2DM and controls

$*=p<0.05$ between T2DM and controls 
Figure 2: Changes in insulin sensitivity upon Acipimox treatment
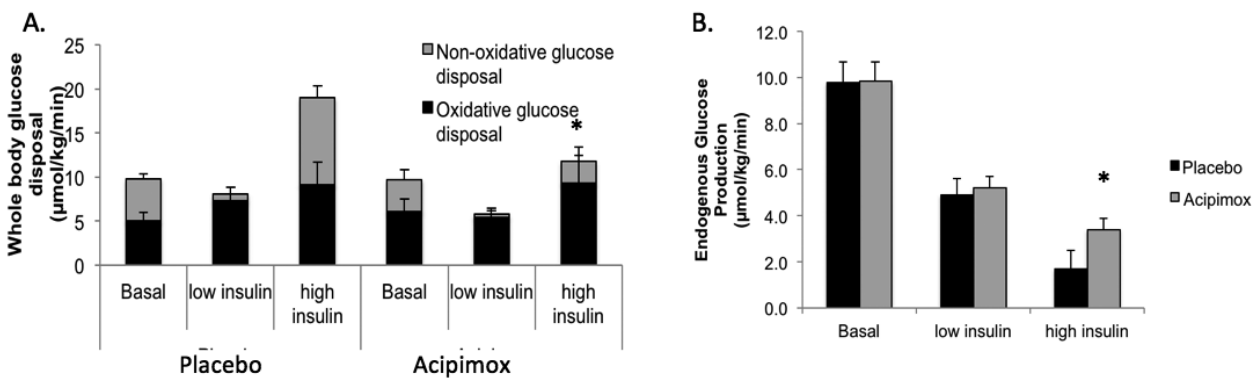

A. whole body glucose disposal rates (WGD) for placebo versus Acipimox treatment in T2D patients. In black oxidative and in grey non-oxidative glucose disposal (NOGD) rates. B. Endogenous glucose production for both placebo and acipimox treated T2D patients. Significant differences $p<0.05$ are indicated by *.

\section{Figure 3: Effects of Acipimox treatment on ectopic fat accumulation}

A.

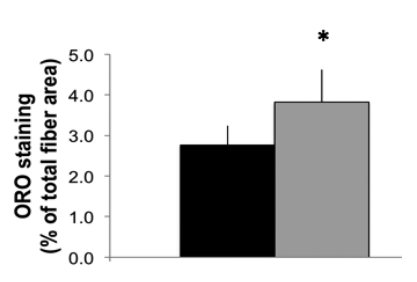

B.

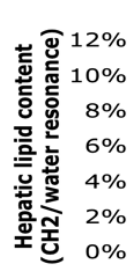

C.

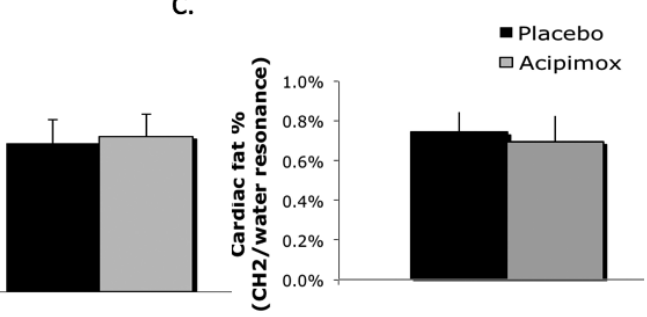

Lipid content measured in T2D patients after either placebo or acipimox treatment. A. Lipid content of skeletal muscle, as measured by ORO staining in $\mathrm{m}$. vastus lateralis. B. lipid content of liver, as measured by ${ }^{1} \mathrm{H}-\mathrm{MRS}$. C. lipid content of heart as measured in 10 subjects with ${ }^{1} \mathrm{H}-\mathrm{MRS}$. Significant differences $\mathrm{p}<0.05$ are indicated by ${ }^{*}$.

\section{Figure 4: Cardiac function and energy status}

A.

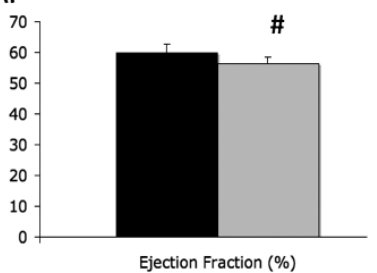

B.

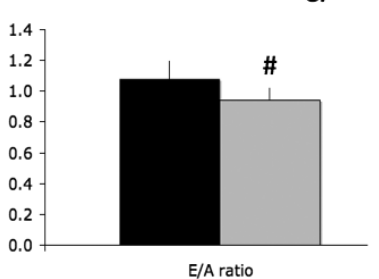

C.

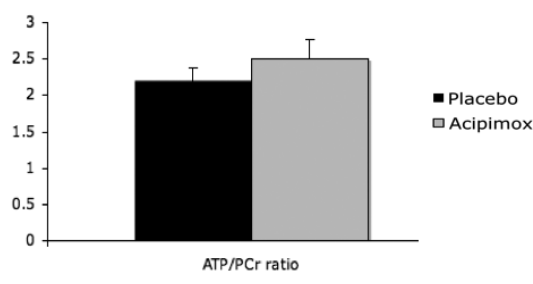

Cardiac function and cardiac energy status measured in 10 T2D subjects. A. Systolic function expressed by Ejection fraction measured by Ultrasound $B$. diastolic function expressed by $E / A$ ratios as measured by Ultrasound $C$. cardiac energy status expressed as PCr/ATP ratio, as measured by ${ }^{31}$ P-MRS. Significant differences $p<0.05$ are indicated by *, $p<0.10$ is indicated by \#. 


\section{Mitochondrial capacity}

All data for PCr-recovery were measured at similar depletion and post-exercise $\mathrm{pH}$ levels. T2D patients $(n=21)$ tended to have a longer $P C r$-recovery half-time than controls, indicating reduced in vivo mitochondrial capacity (PCr-t1/2: $22.2 \pm 1.8 \mathrm{sec}$ vs. $27.4 \pm 2.6 \mathrm{sec}, p=0.08$ ), Upon Acipimox treatment, PCr-recovery half-time increased, suggesting a reduction in in vivo mitochondrial capacity $(27.4 \pm 2.6 \mathrm{sec}$ vs. $30.2 \pm 2.6 \mathrm{sec}$ in placebo vs. Acipimox, $p=0.008$, figure $5 \mathrm{~A})$.

Mitochondrial density as determined by mtDNA copy number, was lower in T2D patients compared to obese control subjects (3555 $\pm 435 \mathrm{AU}$ vs. $2305 \pm 218 \mathrm{AU}, p=0.001)$. However, mtDNA copy number was unaffected by Acipimox treatment $(2305 \pm 218$ AU vs. $2353 \pm 202$ $\mathrm{AU}, p=0.84$, in placebo vs. Acipimox).

Ex vivo mitochondrial function measured as respiration in permeabilized muscle fibers was lower in T2D patients, compared to controls (data not shown). After correction of the mitochondrial respiration for mtDNA content, all differences between T2D patients and controls disappeared (data not shown). In contrast to in vivo findings, ex vivo respiration of mitochondria increased upon Acipimox treatment both for state 3 and for maximal uncoupled respiration on malate/succinate (see figure 5B). Upon addition of octanoyl-carnitine only maximal uncoupled respiration was significantly higher in the Acipimox treated condition (see figure $5 \mathrm{C}$ ). As mtDNA content was not different between the interventions, after correction of mitochondrial respiration for mtDNA content a similar trend with an increased mitochondrial respiration upon Acipimox treatment was observed (data not shown). 
Figure 5: Mitochondrial function
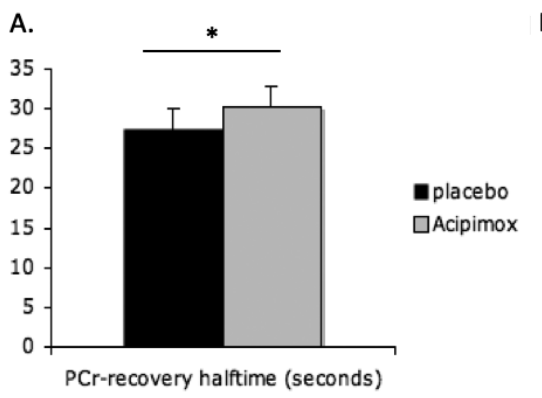

B.

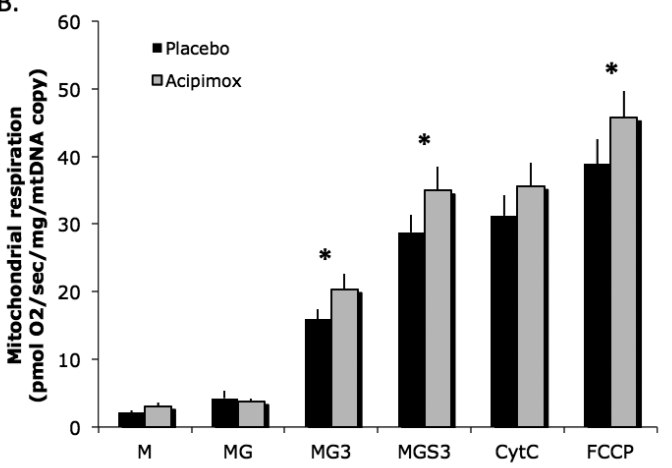

C.

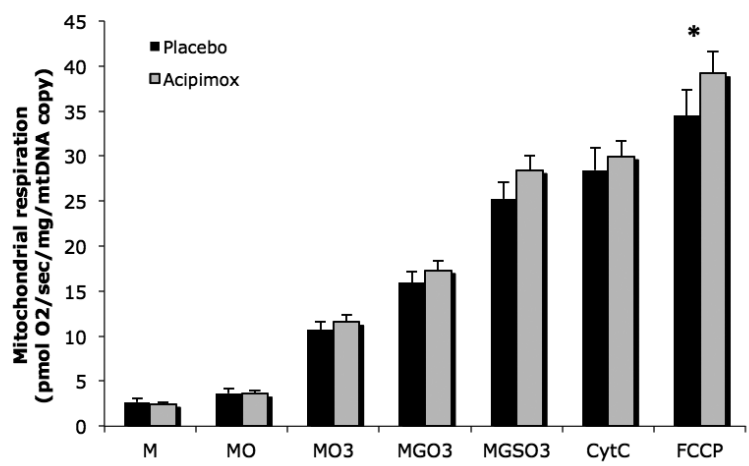

A. in vivo mitochodondrial function as PCr-recovery rate, B. ex vivo mitochondrial function was measured in permeabilized fibers, upon addition of complex I and II substrates uncorrected for mtDNA, in C. the same measurement is repeated, only after adding octanoyl-carnitine as a substrate uncorrected for $m t D N A, M=m a l a t e$, $\mathrm{G}=$ glutamate, $\mathrm{S}=$ succinate, $\mathrm{O}=$ octanoyl-carnitin, $3=$ state 3 after addition of $\mathrm{ADP}, \mathrm{cytC}=$ cytochrome $\mathrm{C}$ FCCP=maximal respiration upon chemical uncoupling with FCCP. Significant differences with $p<0.05$ are indicated by $*$ and $p<0.10$ is indicated by \#. 


\section{Discussion}

The standard clinical dosage of Acipimox only gradually lowered plasma cholesterol and triglyceride concentrations and caused a rebound effect in fasting plasma FFA concentrations, rising up to 2 -fold, after 2 weeks of treatment. This elevation of plasma FFA concentration was paralleled by reduced insulin sensitivity, an increase in skeletal muscle lipid accumulation and decreased in vivo, but not ex vivo mitochondrial function. Furthermore, the elevation of plasma FFA concentration by Acipimox tended to decrease cardiac function in T2D patients.

The observation of elevated FFA levels and impaired insulin action is in contrast to some other studies that did show a long-term reduction of plasma FFA concentrations by Acipimox ${ }^{9,29,53}$. There are 2 major differences between these studies and our study. First, we administered Acipimox 3 times a day, after every meal, according to clinical and medicinal guidelines, whereas other studies used a 4 times daily dosing. Second, in all the previous studies, the last dose of Acipimox was given on the test-day directly prior to blood sampling and analysis. Of note, Acipimox treatment can lead to a rebound effect with plasma FFA rising in the morning, even with a 4 times daily dosage ${ }^{54}$. Saloranta et al. ${ }^{54,55}$ showed that the administration of Acipimox resulted in a sustained daytime rise in plasma FFA, and did not change plasma FFA concentrations over a 24 hour period. It was suggested that this compensatory free fatty acid rise may be necessary to maintain energy production. This notion is of interest, as it is known that mainly nocturnal concentrations of plasma fatty acids are elevated in T2D individuals ${ }^{56}$, with insulin resistance being more pronounced in the morning than in the afternoon ${ }^{57,58}$. Hence, the increase in plasma FFA concentrations upon Acipimox administration is most likely a reflection of a noctural rebound effect, which in our study became apparent due to lack of Acipimox administration in the morning of the experimental test days. Thus, it is likely that previous reports on the effect of Acipimox on plasma FFA concentrations and insulin sensitivity actually reflect the remnant effect of the last dose of Acipimox taken, rather than being a reflection of prolonged Acipimox treatment.

In addition to the absence of a FFA-lowering effect of Acipimox, our study revealed some other interesting findings. The Acipimox-induced elevation of plasma FFA induced lipid accumulation and insulin resistance, and tended to compromise cardiac function in patients with type 2 diabetes. However, cardiac lipid content was not increased, suggesting that plasma FFA concentrations may have a direct, detrimental effect on cardiac function, which is independent of cardiac fat accumulation. Consistently in the study of Letho et al. ${ }^{30}$, changes in lipid supply rather than in lipid accumulation seemed to determine cardiac function and workload. These data suggest that the heart is more susceptible for changes in substrate 
supply rather than changes in cardiac lipid content itself. Furthermore, our data suggest that one should be cautious when treating hyperlipidemia with Acipimox in T2D patients. Future studies should be performed to assess safety and efficacy of Acipimox treatment in T2D patients.

The rebound rise in plasma FFA also provided a model to study the effects of elevated plasma FFA on cardiac, liver and skeletal muscle lipid accumulation and function, without directly altering energy balance, which is the case in lipid infusion or high-fat diet models used to examine the effect of FFA elevation on insulin sensitivity and related parameters. It is thus of interest to note that, in accordance with the lipid infusion paradigms, Acipimox-induced elevation of plasma FFA concentrations also resulted in accumulation of fat in skeletal muscle and insulin resistance. However, in our study, elevated plasma FFA concentrations did not increase liver fat content but did impair hepatic insulin sensitivity. Consistently, a study with Acipimox that resulted in reducing plasma fatty acids found unchanged hepatic fat content, whilst hepatic glucose output was improved ${ }^{31}$. These data together suggest that circulating fatty acids may play a dominant role in regulating hepatic insulin sensitivity independent of hepatic fat content.

We, and others, have previously shown that acute elevation of plasma FFA may decrease mitochondrial biogenesis and function ${ }^{24,26,59-61}$. Rather interestingly, we observed that elevated plasma FFA concentrations decreased in vivo mitochondrial function, but increased ex vivo mitochondrial function. The reduction in in vivo mitochondrial function is consistent with previous findings that elevation of FFA concentrations can reduce mitochondrial metabolism ${ }^{59-62}$. However, it should be noted that in vivo mitochondrial function - as determined by $\mathrm{PCr}$ recovery halftime - is determined not only by intrinsic mitochondrial function, but also by mitochondrial density and perfusion. Therefore, ex vivo mitochondrial function is a more direct measure of skeletal muscle mitochondrial oxidative capacity. As we previously showed that high FFA concentrations - induced by prolonged fasting - resulted in reduction (rather than an improvement) in mitochondrial function ${ }^{61}$, the present results are surprising. Most likely, these findings can be explained by a direct effect of Acipimox on skeletal muscle mitochondrial function. Acipimox is a nicotinic acid analogue, and may thereby increase intracellular NAD+ concentrations in skeletal muscle. Recent reports show that stimulation of the NAD+ biosynthesis pathway, for example by nicotinamide riboside, has beneficial effects on mitochondrial function ${ }^{63}$. Thus, although Acipimox did not have beneficial effects on in vivo mitochondrial function, probably due to negative effects of 
high FFA concentrations on muscle perfusion or substrate delivery, our results suggest that other compounds that may stimulate NAD+ metabolism without increasing plasma FFA concentrations may be very interesting targets for the treatment of mitochondrial dysfunction that is associated with type 2 diabetes.

In conclusion, a two week intervention with clinical dosages of Acipimox led to a rebound rise in plasma FFA, which negatively impacts insulin sensitivity and cardiac function. The decrease in insulin sensitivity was probably mediated through an increase in skeletal muscle lipid accumulation and a decreased in vivo mitochondrial function. Future research is needed to investigate the safety and efficacy of Acipimox treatment for hyperlipidemia in T2D patients. 


\section{References}

1. Krolewski, A.S., Czyzyk, A., Janeczko, D. \& Kopczynski, J. Mortality from cardiovascular diseases among diabetics. Diabetologia 13, 345-50 (1977).

2. Roden, M. et al. Mechanism of free fatty acid-induced insulin resistance in humans. J Clin Invest 97, 2859-65 (1996).

3. Panzram, G. Mortality and survival in type 2 (non-insulin-dependent) diabetes mellitus. Diabetologia 30, 123-31 (1987).

4. Tunaru, S. et al. PUMA-G and HM74 are receptors for nicotinic acid and mediate its antilipolytic effect. Nat Med 9, 352-5 (2003).

5. Vaag, A. et al. Effect of the antilipolytic nicotinic acid analogue acipimox on whole-body and skeletal muscle glucose metabolism in patients with non-insulin-dependent diabetes mellitus. J Clin Invest 88, 1282-90 (1991).

6. Sirtori, C.R. et al. Reduced triglyceridemia and increased high density lipoprotein cholesterol levels after treatment with acipimox, a new inhibitor of lipolysis. Atherosclerosis 38, 267-71 (1981).

7. Jung, W., Kohlmeier, M., Nikolaus, T. \& Schlierf, G. Effects of Acipimox on plasma lipids and biliary lipids in healthy subjects. Res Exp Med (Berl) 185, 457-68 (1985).

8. Taskinen, M.R. \& Nikkila, E.A. Effects of acipimox on serum lipids, lipoproteins and lipolytic enzymes in hypertriglyceridemia. Atherosclerosis 69, 249-55 (1988).

9. Bajaj, M. et al. Effect of a sustained reduction in plasma free fatty acid concentration on intramuscular long-chain fatty Acyl-CoAs and insulin action in type 2 diabetic patients. Diabetes 54, 3148-53 (2005).

10. Bajaj, M. et al. Paradoxical changes in muscle gene expression in insulin-resistant subjects after sustained reduction in plasma free fatty acid concentration. Diabetes 56, 743-52 (2007).

11. van Loon, L.J. et al. Inhibition of adipose tissue lipolysis increases intramuscular lipid use in type 2 diabetic patients. Diabetologia 48, 2097-107 (2005).

12. van Herpen, N.A. \& Schrauwen-Hinderling, V.B. Lipid accumulation in non-adipose tissue and lipotoxicity. Physiol Behav 94, 231-41 (2008).

13. Roden, M. et al. Rapid impairment of skeletal muscle glucose transport/phosphorylation by free fatty acids in humans. Diabetes 48, 358-64 (1999).

14. Krebs, M. et al. Free fatty acids inhibit the glucose-stimulated increase of intramuscular glucose-6-phosphate concentration in humans. The Journal of clinical endocrinology and metabolism 86, 2153-60 (2001).

15. Shulman, G.I. Cellular mechanisms of insulin resistance. J Clin Invest 106, 171-6 (2000).

16. Cusi, K. Role of insulin resistance and lipotoxicity in non-alcoholic steatohepatitis. Clinics in liver disease 13, 545-63 (2009).

17. Boudina, S. \& Abel, E.D. Diabetic cardiomyopathy revisited. Circulation 115, 3213-23 (2007).

18. van de Weijer, T., Schrauwen-Hinderling, V.B. \& Schrauwen, P. Lipotoxicity in type 2 diabetic

19. Phielix, E. et al. Lower intrinsic ADP-stimulated mitochondrial respiration underlies in vivo mitochondrial dysfunction in muscle of male type 2 diabetic patients. Diabetes 57, 2943-9 (2008).

20. Kelley, D.E., He, J., Menshikova, E.V. \& Ritov, V.B. Dysfunction of mitochondria in human skeletal muscle in type 2 diabetes. Diabetes 51, 2944-50 (2002). 
21. He, J., Watkins, S. \& Kelley, D.E. Skeletal muscle lipid content and oxidative enzyme activity in relation to muscle fiber type in type 2 diabetes and obesity. Diabetes 50, 817-23 (2001).

22. Schrauwen-Hinderling, V.B. et al. Impaired in vivo mitochondrial function but similar intramyocellular lipid content in patients with type 2 diabetes mellitus and BMI-matched control subjects. Diabetologia 50, 113-20 (2007).

23. Petersen, K.F., Dufour, S., Befroy, D., Garcia, R. \& Shulman, G.I. Impaired mitochondrial activity in the insulin-resistant offspring of patients with type 2 diabetes. The New England journal of medicine 350, 664-71 (2004).

24. Brehm, A. et al. Increased lipid availability impairs insulin-stimulated ATP synthesis in human skeletal muscle. Diabetes 55, 136-40 (2006).

25. Schrauwen, P., Schrauwen-Hinderling, V., Hoeks, J. \& Hesselink, M.K. Mitochondrial dysfunction and lipotoxicity. Biochim Biophys Acta 1801, 266-71 (2010).

26. Brehm, A. et al. Acute elevation of plasma lipids does not affect ATP synthesis in human skeletal muscle. Am J Physiol Endocrinol Metab 299, E33-8 (2010).

27. Lim, E.L., Hollingsworth, K.G., Smith, F.E., Thelwall, P.E. \& Taylor, R. Inhibition of lipolysis in Type 2 diabetes normalizes glucose disposal without change in muscle glycogen synthesis rates. Clinical science 121, 169-77 (2011).

28. Serlie, M.J. et al. Short-term manipulation of plasma free fatty acids does not change skeletal muscle concentrations of ceramide and glucosylceramide in lean and overweight subjects. The Journal of clinical endocrinology and metabolism 92, 1524-9 (2007).

29. Bajaj, M. et al. Sustained reduction in plasma free fatty acid concentration improves insulin action without altering plasma adipocytokine levels in subjects with strong family history of type 2 diabetes. J Clin Endocrinol Metab 89, 4649-55 (2004).

30. Lehto, H.R. et al. Effects of acute and one-week fatty acid lowering on cardiac function and insulin sensitivity in relation with myocardial and muscle fat and adiponectin levels. J Clin Endocrinol Metab 97, 3277-84 (2012).

31. Rigazio, S. et al. The lowering of hepatic fatty acid uptake improves liver function and insulin sensitivity without affecting hepatic fat content in humans. Am J Physiol Endocrinol Metab 295, E413-9 (2008).

32. Kuipers, H., Verstappen, F.T., Keizer, H.A., Geurten, P. \& van Kranenburg, G. Variability of aerobic performance in the laboratory and its physiologic correlates. Int J Sports Med 6, 197201 (1985).

33. Siri, W.E. The gross composition of the body. Adv Biol Med Phys 4, 239-80 (1956).

34. DeFronzo, R.A., Tobin, J.D. \& Andres, R. Glucose clamp technique: a method for quantifying insulin secretion and resistance. Am J Physiol 237, E214-23 (1979).

35. Mensink, M., Blaak, E.E., van Baak, M.A., Wagenmakers, A.J. \& Saris, W.H. Plasma free Fatty Acid uptake and oxidation are already diminished in subjects at high risk for developing type 2 diabetes. Diabetes 50, 2548-54 (2001).

36. Frayn, K.N. Calculation of substrate oxidation rates in vivo from gaseous exchange. J Appl Physiol 55, 628-34 (1983).

37. Bergstrom, J., Hermansen, L., Hultman, E. \& Saltin, B. Diet, muscle glycogen and physical performance. Acta Physiol Scand 71, 140-50 (1967).

38. Koopman, R., Schaart, G. \& Hesselink, M.K. Optimisation of oil red O staining permits combination with immunofluorescence and automated quantification of lipids. Histochem Cell Biol 116, 63-8 (2001). 
39. Veksler, V.I., Kuznetsov, A.V., Sharov, V.G., Kapelko, V.I. \& Saks, V.A. Mitochondrial respiratory parameters in cardiac tissue: a novel method of assessment by using saponin-skinned fibers. Biochim Biophys Acta 892, 191-6 (1987).

40. Phielix, E. et al. High oxidative capacity due to chronic exercise training attenuates lipidinduced insulin resistance. Diabetes 61, 2472-8 (2012).

41. Timmers, S. et al. Calorie restriction-like effects of 30 days of resveratrol supplementation on energy metabolism and metabolic profile in obese humans. Cell Metab 14, 612-22 (2012).

42. Vanhamme, L., van den Boogaart, A. \& Van Huffel, S. Improved method for accurate and efficient quantification of MRS data with use of prior knowledge. J Magn Reson 129, 35-43 (1997).

43. Naressi, A. et al. Java-based graphical user interface for the MRUI quantitation package. Magma 12, 141-52 (2001).

44. Hamilton, G. et al. In vivo characterization of the liver fat (1)H MR spectrum. NMR Biomed 24, 784-90 (2011).

45. Schrauwen-Hinderling, V.B. et al. Improved ejection fraction after exercise training in obesity is accompanied by reduced cardiac lipid content. J Clin Endocrinol Metab 95, 1932-8 (2010).

46. van der Meer, R.W. et al. Metabolic imaging of myocardial triglyceride content: reproducibility of $1 \mathrm{H}$ MR spectroscopy with respiratory navigator gating in volunteers. Radiology 245, 251-7 (2007).

47. Kozerke, S., Schar, M., Lamb, H.J. \& Boesiger, P. Volume tracking cardiac 31P spectroscopy. Magn Reson Med 48, 380-4 (2002).

48. Yagyu, H. et al. Lipoprotein lipase ( $\mathrm{LpL}$ ) on the surface of cardiomyocytes increases lipid uptake and produces a cardiomyopathy. J Clin Invest 111, 419-26 (2003).

49. Bilet, L. et al. Exercise-induced modulation of cardiac lipid content in healthy lean young men. Basic Res Cardiol 106, 307-15 (2011).

50. Lamb, H.J. et al. Reproducibility of human cardiac 31P-NMR spectroscopy. NMR Biomed 9, 217-27 (1996).

51. Hansch, A. et al. Noninvasive measurements of cardiac high-energy phosphate metabolites in dilated cardiomyopathy by using 31P spectroscopic chemical shift imaging. Eur Radiol 15, 319-23 (2005).

52. Steele, R. Influences of glucose loading and of injected insulin on hepatic glucose output. Ann N Y Acad Sci 82, 420-30 (1959).

53. Worm, D. et al. Pronounced blood glucose-lowering effect of the antilipolytic drug acipimox in noninsulin-dependent diabetes mellitus patients during a 3-day intensified treatment period. J Clin Endocrinol Metab 78, 717-21 (1994).

54. Saloranta, C. et al. Metabolic consequences of sustained suppression of free fatty acids by acipimox in patients with NIDDM. Diabetes 42, 1559-66 (1993).

55. Saloranta, C. et al. Different acute and chronic effects of acipimox treatment on glucose and lipid metabolism in patients with type 2 diabetes. Diabet Med 10, 950-7 (1993).

56. Reaven, G.M., Hollenbeck, C., Jeng, C.Y., Wu, M.S. \& Chen, Y.D. Measurement of plasma glucose, free fatty acid, lactate, and insulin for $24 \mathrm{~h}$ in patients with NIDDM. Diabetes 37 , 1020-4 (1988).

57. Bolli, G.B. The dawn phenomenon: its origin and contribution to early morning hyperglycemia in diabetes mellitus. Diabete Metab 14, 675-86 (1988).

58. Shapiro, E.T. et al. Nocturnal elevation of glucose levels during fasting in noninsulindependent diabetes. J Clin Endocrinol Metab 72, 444-54 (1991). 
59. Hoeks, J. et al. Peroxisome proliferator-activated receptor-gamma coactivator-1 and insulin resistance: acute effect of fatty acids. Diabetologia 49, 2419-26 (2006).

60. Groop, L.C., Bonadonna, R.C., Shank, M., Petrides, A.S. \& DeFronzo, R.A. Role of free fatty acids and insulin in determining free fatty acid and lipid oxidation in man. $J$ Clin Invest 87, 83-9 (1991).

61. Hoeks, J. et al. Prolonged fasting identifies skeletal muscle mitochondrial dysfunction as consequence rather than cause of human insulin resistance. Diabetes 59, 2117-25 (2010).

62. Chavez, A.O. et al. Effect of short-term free Fatty acids elevation on mitochondrial function in skeletal muscle of healthy individuals. J Clin Endocrinol Metab 95, 422-9 (2010).

63. Canto, C. et al. The $\mathrm{NAD}(+)$ precursor nicotinamide riboside enhances oxidative metabolism and protects against high-fat diet-induced obesity. Cell Metab 15, 838-47 (2012). 


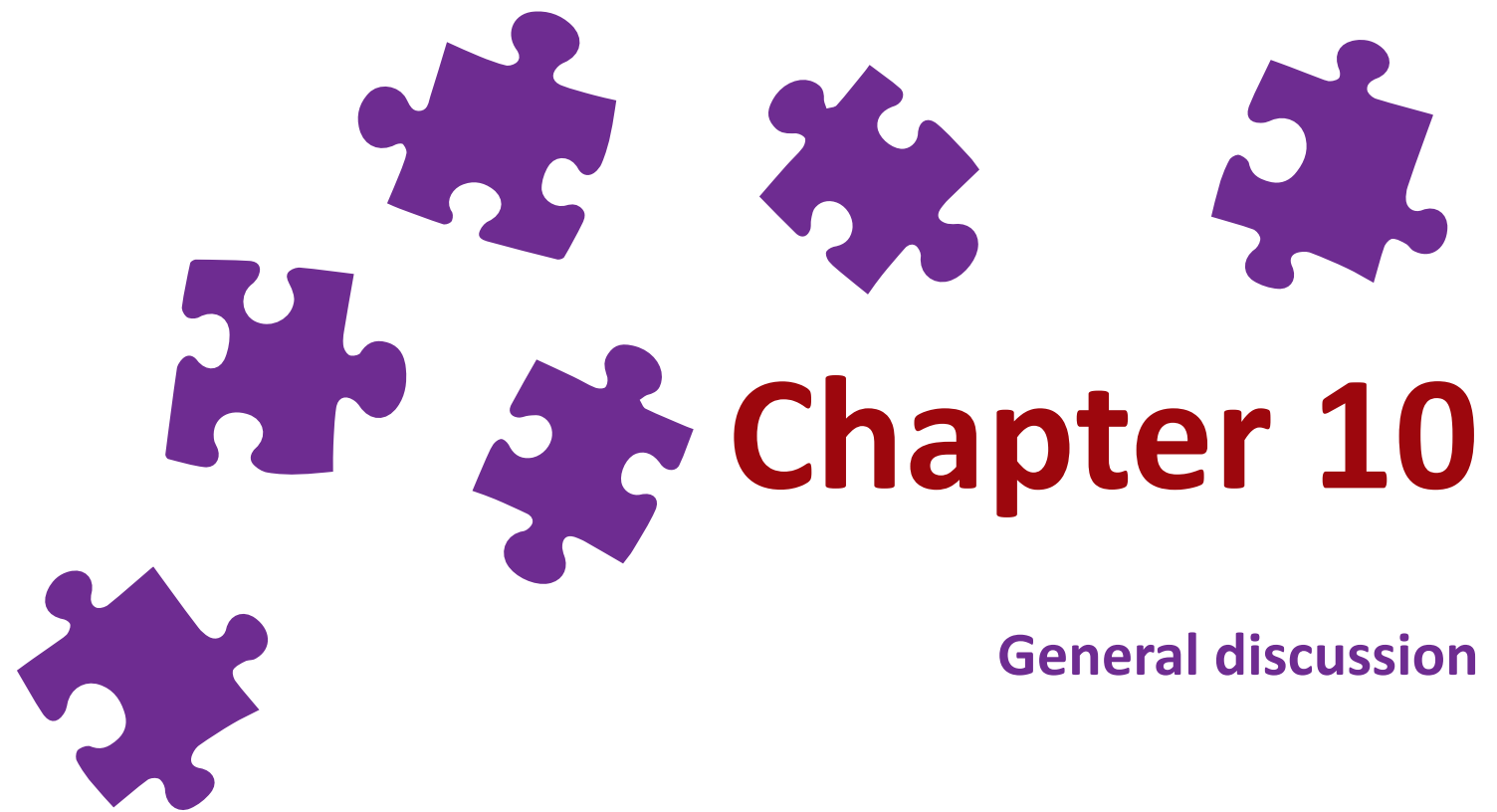

General discussion 
Historically, type 2 diabetes has been described as a disease characterized by deranged glucose metabolism, causing frequent urination and sweet (honey-like) urine. Indeed, diabetes is diagnosed by elevated plasma glucose levels. However, nowadays we know that type 2 diabetes is not only characterized by a deranged glucose metabolism, but also by a deranged lipid metabolism. Typically type 2 diabetic patients are characterized by an increased lipid content in ectopic tissues, which has been associated with the development of insulin resistance ${ }^{1-3}$, and a decreased oxidative capacity ${ }^{4-8}$. This might suggest that ectopic fat accumulation per se is detrimental. However, one can question this concept as endurance trained athletes, who have an increased fat accumulation in skeletal muscle are metabolically healthy and insulin sensitive ${ }^{9}$. Thus, the aim of this thesis was to unravel both the causes and consequences of ectopic fat accumulation in the development of type 2 diabetes.

\section{1) Does manipulation of the plasma free fatty acid concentration lead to an altered cardiac or skeletal muscle fat content?}

Fat accumulation in non-adipose tissues as observed in diabetic patients has been suggested to be due to elevated circulating plasma free fatty acids (FFA). In this thesis we have manipulated plasma FFA concentrations both physiologically with exercise (chapter 8) and pharmacologically with Acipimox (chapter 9). Upon exercise in the fasting condition, plasma FFA concentrations were elevated, which resulted in cardiac fat accumulation in lean young men. The latter was not a direct effect of exercise, since glucose supplementation during exercise, which blunted the elevation of plasma FFA concentrations, did not result in an increased cardiac lipid accumulation. Therefore, we concluded that acute elevation of plasma FFA levels may result in cardiac fat accumulation. This finding is consistent with other studies in healthy young men as well as in obese type 2 diabetic subjects, in which myocardial lipid content significantly increased upon fasting-induced elevation of plasma FFA concentrations ${ }^{10,11}$. In chapter 9, we attempted to lower plasma FFA concentrations in type 2 diabetic patients with the anti-lipolytic agent Acipimox. Instead of lowering plasma FFA concentrations, prolonged ( 2 weeks) administration of Acipimox induced a rebound effect causing a raise in circulating plasma FFA concentrations. Under these circumstances, however, the elevation in plasma FFA concentrations did not promote alterations in cardiac lipid content. Also, in the study of Lehto et al. ${ }^{12}$, where a successful reduction of plasma FFA concentrations was established with Acipimox in young lean subjects, the lowering in plasma FFA concentrations did not result in a change in cardiac lipid content. There are two mayor differences between these studies; first of all, the degree of elevation and suppression of the plasma FFA levels with Acipimox was milder compared to the effects of fasting and excersice. 
Furthermore the studies that do show effects of plasma FFA concentrations on cardiac lipid accumulation also increase the energy demand of the heart. Thus, fasting and exercise both increase fatty acid oxidation rates in the heart, whereas Acipimox does not necessarily lead to a change in cardiac lipid demand. These findings may suggest that not only supply of fatty acids to the tissue of interest but also demand of fatty acids for subsequent oxidation are determinants of cardiac fat accumulation. Hence, changes in plasma fatty acids alone might not necessarily lead to an altered cardiac lipid content. In animals, the fatty acid transporters CD36 and FATP4 are not only under the regulation of fatty acid supply, but also under the regulation of $\mathrm{AMPK}^{13-16}$. This supports the notion that energy demand, in combination with a high FFA supply, seems to be an important factor determining cardiac lipid accumulation.

In chapter 9, we showed that elevating plasma FFA concentrations resulted in skeletal muscle lipid accumulation. Other studies, using Acipimox, successfully lowered plasma free fatty acids when using Acipimox acutely ${ }^{10,17,18}$, chronically ${ }^{12,19,20}$ or even post-exercise ${ }^{21,22}$. Furthermore, the Acipimox-induced lowering of fatty acids in these studies resulted in a reduction in skeletal muscle lipid accumulation. Acute exercise in the fasting condition has also been shown to increase plasma FFA concentrations and skeletal muscle fat accumulation in resting muscles on the short-term ${ }^{23}$. Likewise, lipid infusion increases both plasma TG and skeletal muscle lipid content in healthy lean young men $^{24}$.

Taken together, skeletal muscle lipid accumulation can be augmented by an increase in fatty acid supply, but in contrast to the heart, this seems possible both with and without an increase in energy demand, suggesting that FA uptake may be differently regulated in skeletal versus cardiac muscle.

\section{2) Do alterations in cardiac and skeletal muscle lipid content lead to an altered cardiac function and skeletal muscle insulin sensitivity?}

It has been suggested that an increased cardiac lipid accumulation is associated with the development of diabetic cardiomyopathy ${ }^{25-27}$. Although type 2 diabetes has been associated with an increase in cardiac lipid accumulation, it remains unclear whether cardiac lipid accumulation is functionally related with an impairment of cardiac function.

In chapter 8 , we showed that in healthy young volunteers cardiac fat accumulation is increased during and after exercise in the fasted state, which was accompanied by a slight improvement of cardiac function. Although the improvement in cardiac function most likely reflected the exercise-induced sympathetic drive, which may have been higher when exercise was performed in the fasted state, the results do indicate that fat accumulation per se does not need to be detrimental to organ function. Even more so, it was shown previously 
that an acute lowering of cardiac fat content with Acipimox in patients with heart failure, deteriorated cardiac function rather than preserved $i^{28}$. These data suggest that, at least in heart failure, lowering plasma FFA concentrations acutely might lower the cardiac energy status.

The notion that cardiac lipid content is not a very strong determinant of cardiac function is also strengthened by more long-term studies that manipulate plasma FFA concentrations. Lowering plasma FFA concentrations with Acipimox in young healthy volunteers did not affect cardiac lipid accumulation, but still lowered cardiac function ${ }^{12}$. Results from studies with patients undergoing bariatric surgery show that even with an extreme weight loss no changes in cardiac lipid accumulation could be achieved, but cardiac function improved slightly ${ }^{29,30}$. Therefore, the manipulation of plasma FFA concentrations might not always predict changes in cardiac function. Consistently, we showed that elevation of plasma free fatty acids due to an Acipimox-induced rebound effect, in type 2 diabetic patients did also not alter cardiac lipid accumulation, but tended to lower cardiac function (chapter 9). These data suggest that the heart of type 2 diabetic patients is very sensitive to fluctuations in plasma FFA concentrations rather than changes in cardiac fat accumulation.

We also used Adipose Triglyceride Lipase (ATGL) knock-out mice as a model for cardiac fat accumulation: the extreme fat accumulation in the heart that goes along with knocking down the major triacylglycerol lipase, profoundly compromised cardiac function. Surprisingly, however, this mainly seems to be due to the fact that the knock-down of ATGL led to reduced generation of essential mediator(s) involved in the generation of lipid-droplet derived FFA as ligands for peroxisome proliferator-activated receptor (PPAR) activation, resulting in impairment in oxidative metabolism (chapter 4). Treating ATGL deficient mice with synthetic PPAR agonists resulted in a normalization of mRNA levels of PPAR- $\alpha$ and PPAR- $\delta$ target genes and completely reversed the mitochondrial defects, restored cardiac function, reduced cardiac lipid content and prevented premature death. In humans, Neutral Lipid Storage Disease (NLSDM) is also caused by a defect in the gene encoding ATGL, and patients phenotypically present with a phenotype similar to ATGL knockout mice. In this thesis, we showed that indeed oxidative metabolism (mainly fatty acid oxidation) - albeit measured in muscle - was low in these patients and that this could be normalized after treatment with Bezafibrate (a pharmacological PPAR agonist) for 28 weeks. This also resulted in a lowering of cardiac fat content. However, although metabolic changes became apparent, this trial did not (yet) result in changes in clinically relevant parameters, most likely due to the short duration of the study. Further research is warranted in order to establish whether a disturbed lipolysis of ectopic fat can also be of importance in diabetic cardiomyopathy. 
In skeletal muscle it is known for some time now that lipid accumulation does not need to cause insulin resistance per se. This was best described by Goodpaster et al. ${ }^{9}$, who showed that highly trained athletes had an increased lipid accumulation in skeletal muscle, yet were very insulin sensitive. Indeed, exercise training has been shown to improve insulin sensitivity and fat oxidative capacity, despite an increase in skeletal muscle fat content ${ }^{31}$. This indicates that skeletal muscle lipid accumulation does not need to be detrimental per se. Still, there are many studies pointing towards a relationship between skeletal muscle lipid accumulation in obesity and insulin resistance ${ }^{1-3,32}$. However, when subjects are well matched for age and BMI, differences in skeletal muscle lipid accumulation between type 2 diabetic patients and healthy controls are inconsistent ${ }^{33}$. In agreement, in chapter 7 we also did not detect any differences in muscle fat content in a large cohort of well-matched type 2 diabetic and control subjects. Yet, (by design) there was a vast difference in insulin sensitivity. These results further indicate that skeletal muscle lipid accumulation is not the primary cause of insulin resistance.

Nevertheless, the results of the present thesis do not exclude a role for lipid accumulation in the development of insulin resistance. For example, in chapter 9 the Acipimox-induced increase in fatty acid supply was paralleled by increased skeletal muscle lipid content and insulin resistance. Many other studies using fasting ${ }^{6}$, lipid infusion ${ }^{34-36}$ or high fat diets ${ }^{37-39}$ have established this notion before. Jointly, these results indicate that the combination of a surplus of fatty acid supply, lipid accumulation in skeletal muscle and unadjusted oxidation rates or energy expenditure can induce insulin resistance. In particular, the balance between muscle fat content and fat oxidative capacity may be important, as it has been shown that skeletal muscle mitochondrial function in type 2 diabetes is impaired ${ }^{7,33,40}$ (also see chapter 7 and 9).

\section{3) Can cardiac fat content be linked to an altered cardiac energy status?}

Cardiac energy status can be measured with ${ }^{31} \mathrm{P}-\mathrm{MRS}$ and is expressed as the ratio of Phosphocreatine over ATP (PCr/ATP ratio). Some studies have shown that cardiac energy status is lower in patients with type 2 diabetes ${ }^{40-42}$, suggesting impaired mitochondrial function, which might be causally related to the development of type 2 diabetic cardiomyopathy. It has also been suggested that this impairment in mitochondrial function might be related to either an increased cardiac lipid accumulation or an increased FFA supply ${ }^{40}$. This theory fits with the results found in chapter 8 . Thus, an increase in cardiac fat accumulation upon exercise in the fasted state was paralleled by a decrease in cardiac energy status. However, cardiac function improved rather than decreased. Thus, although the lower cardiac energy status is in line with a lipotoxic action of cardiac lipid content, a relationship with an impaired 
cardiac function could not be found in this study, but it should be kept in mind that this study was a short intervention study in healthy subjects. Furthermore an elevation in plasma FFA concentrations in chapter 9 , neither changed cardiac fat accumulation nor cardiac energy status, but did result in a tendency towards a decreased cardiac function. These results do not point towards causal relationship between cardiac lipid accumulation and energy status in the development of diabetic cardiomyopathy, at least not within the time frame of our studies. Accordingly, other studies also do not find any relationships between cardiac lipid accumulation $^{43}$ and energy status ${ }^{25,44}$ in the hearts of type 2 diabetic patients.

In the study of Neubauer et al. ${ }^{45}$ a strong relationship between cardiac energy status and prognosis in patients with heart failure was found. Thus, a PCr/ATP ratio of lower than 1.6 appeared to be associated with a poor prognosis. Although some studies ${ }^{40}$ do show an extremely low cardiac energy status in metabolically deranged type 2 diabetic patients, most studies ${ }^{44}$ including our studies do not report PCr/ATP ratios of 1.6 or lower in type 2 diabetic patients under good glycemic control, and therefore these values are probably well within a normal physiological range and may not have consequences for cardiac function. The study of Scheuerman-Freestone et al. ${ }^{40}$ also showed that PCr/ATP ratios positively correlated with plasma glucose and negatively correlated with plasma free fatty acids. Hence, changes observed in PCr/ATP ratios upon intervention in our studies (chapter 8 and 9) might be solely linked to alterations in substrate supply and metabolism, rather than reflect an energy deficit of the heart. Thus, although PCr/ATP ratio may be affected by fatty acid supply, it remains to be elucidated whether this should be interpreted as a pathophysiological adaptation.

\section{4) Future perspectives}

We showed in this thesis that elevation or suppression of plasma FFA concentrations does not necessarily lead to alterations in cardiac lipid accumulation. Cardiac lipid accumulation could also not be causally linked to alterations in cardiac function. However, cardiac function in type 2 diabetes did seem to be very sensitive to fluctuations in cardiac substrate supply. Manipulation of fatty acid transporters like for instance CD36 and FATP4 in vitro and maybe also in vivo could be valuable in order to assess the effects of changes in fatty acid supply on cardiac function. This might eventually even translate into new cardioprotective therapies in the future.

Also the PPAR-pathways are known to be involved in substrate uptake and oxidation. Although in the past, these pathways have been targeted therapeutically in type 2 diabetes with the Thiazolidinediones (TZDs), these therapies were mainly focused on maintaining 
glycemic control, rather than cardiac protection. Indeed glycemic control has been identified as an important factor determining cardiac health, but most of these TZDs are mainly PPAR- $\gamma$ agonists and PPAR- $\gamma$ is mainly expressed in adipose tissue. Furthermore it was shown that treatment with some of these TZDs lead to fluid retention and oedema, which was associated with an increased risk for heart failure. Hence safety and efficiency of TZDs in diabetic patients at risk of cardiovasculular disease is still under debate.

Fibrates (PPAR- $\alpha / \delta$ agonists) have been prescribed as lipid lowering therapy in type 2 diabetes and have been shown to be cardio-protective. Until now, we have assumed that this effect was merely caused by reducing atherosclerosis. However it might be that fibrates have a direct effect on the heart via activation of the PPAR- $\alpha$ and $\delta$ target genes in type 2 diabetes. This has never been properly investigated in humans. Furthermore, it remains unclear whether PPAR-expression in the heart is altered in type 2 diabetes. Hence, investigating PPAR expression and manipulation of these PPARs in type 2 diabetic cardiomyopathy might be of value for future research.

As it was shown that mitochondria might also play a role in metabolic flexibility in type 2 diabetes, targeting mitochondrial biogenesis and function might also contribute to metabolic health in type 2 diabetes. However, although a lot of research is done on mitochondrial function in skeletal muscle, little is known about mitochondrial function of the heart. Hence, studies assessing cardiac mitochondrial function in a broad variety of subjects, might give more insight in the role of mitochondrial function in the development of diabetic cardiomyopathy. As it is difficult to collect tissue from the heart, there is a need for non-invasive measurements of mitochondrial function of the heart. It has been suggested that measuring cardiac energy status with ${ }^{31} \mathrm{P}-\mathrm{MRS}$ might be of value here. However, as discussed above, the meaning of the PCr/ATP ratio remains unclear. Thus, comparing ex vivo measurements for mitochondrial function with the PCr/ATP ratios found in vivo, is needed to validate the relevance of measuring PCr/ATP ratio as a marker of cardiac energy status.

Lastly, we used the ATGL deficient mice as a model for cardiac lipid accumulation. Although this proved to be a poor model for diabetic cardiolipotoxicity, it led to a better understanding of the regulation of mitochondrial function through lipid ligands from the neutral lipid pool. Thus, the lower mitochondrial function in ATGL knockout mice was caused by a lowered availability of lipid ligands liberated from the lipid droplet that could activate PPAR transcription, and this effect could be elegantly circumvented by administration of PPAR-agonists like for instance fibrates. We showed that patients with NLSDM, who are characterized by ATGL mutations, might also benefit from treatment with these fibrates, 
although the clinical translation of these changes remained unclear. Hence, further investigation with more subjects and longer intervention periods is needed to understand the pathological mechanism involved in neutral lipid storage disease. 


\section{References}

1. Jacob S, Machann J, Rett K, Brechtel K, Volk A, Renn W, Maerker E, Matthaei S, Schick F, Claussen CD, Haring HU. Association of increased intramyocellular lipid content with insulin resistance in lean nondiabetic offspring of type 2 diabetic subjects. Diabetes. 1999;48:11131119

2. Sinha R, Dufour S, Petersen KF, LeBon V, Enoksson S, Ma YZ, Savoye M, Rothman DL, Shulman $\mathrm{Gl}$, Caprio S. Assessment of skeletal muscle triglyceride content by (1)h nuclear magnetic resonance spectroscopy in lean and obese adolescents: Relationships to insulin sensitivity, total body fat, and central adiposity. Diabetes. 2002;51:1022-1027

3. Krssak M, Falk Petersen K, Dresner A, DiPietro L, Vogel SM, Rothman DL, Roden M, Shulman GI. Intramyocellular lipid concentrations are correlated with insulin sensitivity in humans: A 1h nmr spectroscopy study. Diabetologia. 1999;42:113-116

4. Kelley DE, He J, Menshikova EV, Ritov VB. Dysfunction of mitochondria in human skeletal muscle in type 2 diabetes. Diabetes. 2002;51:2944-2950

5. Schrauwen P, Schrauwen-Hinderling V, Hoeks J, Hesselink MK. Mitochondrial dysfunction and lipotoxicity. Biochim Biophys Acta. 2010;1801:266-271

6. Hoeks J, van Herpen NA, Mensink M, Moonen-Kornips E, van Beurden D, Hesselink MK, Schrauwen P. Prolonged fasting identifies skeletal muscle mitochondrial dysfunction as consequence rather than cause of human insulin resistance. Diabetes. 2010;59:2117-2125

7. Phielix E, Schrauwen-Hinderling VB, Mensink M, Lenaers E, Meex R, Hoeks J, Kooi ME, Moonen-Kornips E, Sels JP, Hesselink MK, Schrauwen P. Lower intrinsic adp-stimulated mitochondrial respiration underlies in vivo mitochondrial dysfunction in muscle of male type 2 diabetic patients. Diabetes. 2008;57:2943-2949

8. Rabol R, Boushel R, Dela F. Mitochondrial oxidative function and type 2 diabetes. Appl Physiol Nutr Metab. 2006;31:675-683

9. Goodpaster BH, He J, Watkins S, Kelley DE. Skeletal muscle lipid content and insulin resistance: Evidence for a paradox in endurance-trained athletes. J Clin Endocrinol Metab. 2001;86:5755-5761

10. Hammer S, van der Meer RW, Lamb HJ, de Boer HH, Bax JJ, de Roos A, Romijn JA, Smit JW. Short-term flexibility of myocardial triglycerides and diastolic function in patients with type 2 diabetes mellitus. Am J Physiol Endocrinol Metab. 2008;295:E714-718

11. van der Meer RW, Hammer S, Smit JW, Frolich M, Bax JJ, Diamant M, Rijzewijk LJ, de Roos A, Romijn JA, Lamb HJ. Short-term caloric restriction induces accumulation of myocardial triglycerides and decreases left ventricular diastolic function in healthy subjects. Diabetes. 2007;56:2849-2853

12. Lehto HR, Parkka J, Borra R, Tuunanen H, Lepomaki V, Parkkola R, Knuuti J, Nuutila P, Iozzo P. Effects of acute and one-week fatty acid lowering on cardiac function and insulin sensitivity in relation with myocardial and muscle fat and adiponectin levels. $J$ Clin Endocrinol Metab. 2012;97:3277-3284

13. Luiken JJ, Coort SL, Willems J, Coumans WA, Bonen A, van der Vusse GJ, Glatz JF. Contractioninduced fatty acid translocase/cd36 translocation in rat cardiac myocytes is mediated through amp-activated protein kinase signaling. Diabetes. 2003;52:1627-1634

14. Nickerson JG, Alkhateeb H, Benton CR, Lally J, Nickerson J, Han XX, Wilson MH, Jain SS, Snook LA, Glatz JF, Chabowski A, Luiken JJ, Bonen A. Greater transport efficiencies of the 
membrane fatty acid transporters fat/cd36 and fatp4 compared with fabppm and fatp1 and differential effects on fatty acid esterification and oxidation in rat skeletal muscle. J Biol Chem. 2009;284:16522-16530

15. Nickerson JG, Momken I, Benton CR, Lally J, Holloway GP, Han XX, Glatz JF, Chabowski A, Luiken JJ, Bonen A. Protein-mediated fatty acid uptake: Regulation by contraction, ampactivated protein kinase, and endocrine signals. Appl Physiol Nutr Metab. 2007;32:865-873

16. Bonen A, Campbell SE, Benton CR, Chabowski A, Coort SL, Han XX, Koonen DP, Glatz JF, Luiken JJ. Regulation of fatty acid transport by fatty acid translocase/cd36. Proc Nutr Soc. 2004;63:245-249

17. Serlie MJ, Meijer AJ, Groener JE, Duran M, Endert E, Fliers E, Aerts JM, Sauerwein HP. Shortterm manipulation of plasma free fatty acids does not change skeletal muscle concentrations of ceramide and glucosylceramide in lean and overweight subjects. J Clin Endocrinol Metab. 2007; $92: 1524-1529$

18. Lim EL, Hollingsworth KG, Smith FE, Thelwall PE, Taylor R. Inhibition of lipolysis in type 2 diabetes normalizes glucose disposal without change in muscle glycogen synthesis rates. Clin Sci (Lond). 2011;121:169-177

19. Bajaj M, Suraamornkul S, Kashyap S, Cusi K, Mandarino L, DeFronzo RA. Sustained reduction in plasma free fatty acid concentration improves insulin action without altering plasma adipocytokine levels in subjects with strong family history of type 2 diabetes. J Clin Endocrinol Metab. 2004;89:4649-4655

20. Bajaj M, Suraamornkul S, Romanelli A, Cline GW, Mandarino LJ, Shulman GI, DeFronzo RA. Effect of a sustained reduction in plasma free fatty acid concentration on intramuscular longchain fatty acyl-coas and insulin action in type 2 diabetic patients. Diabetes. 2005;54:31483153

21. van Loon LJ, Manders RJ, Koopman R, Kaastra B, Stegen JH, Gijsen AP, Saris WH, Keizer HA. Inhibition of adipose tissue lipolysis increases intramuscular lipid use in type 2 diabetic patients. Diabetologia. 2005;48:2097-2107

22. Tunstall RJ, McAinch AJ, Hargreaves M, van Loon LJ, Cameron-Smith D. Reduced plasma free fatty acid availability during exercise: Effect on gene expression. Eur J Appl Physiol. 2007;99:485-493

23. Schrauwen-Hinderling VB, van Loon LJ, Koopman R, Nicolay K, Saris WH, Kooi ME. Intramyocellular lipid content is increased after exercise in nonexercising human skeletal muscle. J Appl Physiol. 2003;95:2328-2332

24. Itani SI, Ruderman NB, Schmieder F, Boden G. Lipid-induced insulin resistance in human muscle is associated with changes in diacylglycerol, protein kinase c, and ikappab-alpha. Diabetes. 2002;51:2005-2011

25. Rijzewijk LJ, van der Meer RW, Lamb HJ, de Jong HW, Lubberink M, Romijn JA, Bax JJ, de Roos A, Twisk JW, Heine RJ, Lammertsma AA, Smit JW, Diamant M. Altered myocardial substrate metabolism and decreased diastolic function in nonischemic human diabetic cardiomyopathy: Studies with cardiac positron emission tomography and magnetic resonance imaging. J Am Coll Cardiol. 2009;54:1524-1532

26. Kankaanpaa M, Lehto HR, Parkka JP, Komu M, Viljanen A, Ferrannini E, Knuuti J, Nuutila P, Parkkola R, lozzo P. Myocardial triglyceride content and epicardial fat mass in human obesity: Relationship to left ventricular function and serum free fatty acid levels. J Clin Endocrinol Metab. 2006;91:4689-4695 
27. McGavock JM, Lingvay I, Zib I, Tillery T, Salas N, Unger R, Levine BD, Raskin P, Victor RG, Szczepaniak LS. Cardiac steatosis in diabetes mellitus: A 1h-magnetic resonance spectroscopy study. Circulation. 2007;116:1170-1175

28. Tuunanen $\mathrm{H}$, Engblom E, Naum A, Nagren K, Hesse B, Airaksinen KE, Nuutila P, lozzo P, Ukkonen H, Opie LH, Knuuti J. Free fatty acid depletion acutely decreases cardiac work and efficiency in cardiomyopathic heart failure. Circulation. 2006;114:2130-2137

29. Gaborit B, Jacquier A, Kober F, Abdesselam I, Cuisset T, Boullu-Ciocca S, Emungania O, Alessi $M C$, Clement K, Bernard M, Dutour A. Effects of bariatric surgery on cardiac ectopic fat: Lesser decrease in epicardial fat compared to visceral fat loss and no change in myocardial triglyceride content. J Am Coll Cardiol. 2012;60:1381-1389

30. Vest AR, Heneghan HM, Agarwal S, Schauer PR, Young JB. Bariatric surgery and cardiovascular outcomes: A systematic review. Heart. 2012;98:1763-1777

31. Meex RC, Schrauwen-Hinderling VB, Moonen-Kornips E, Schaart G, Mensink M, Phielix E, van de Weijer T, Sels JP, Schrauwen P, Hesselink MK. Restoration of muscle mitochondrial function and metabolic flexibility in type 2 diabetes by exercise training is paralleled by increased myocellular fat storage and improved insulin sensitivity. Diabetes. 2010;59:572579

32. Perseghin G, Scifo P, De Cobelli F, Pagliato E, Battezzati A, Arcelloni C, Vanzulli A, Testolin G, Pozza G, Del Maschio A, Luzi L. Intramyocellular triglyceride content is a determinant of in vivo insulin resistance in humans: A $1 \mathrm{~h}-13 \mathrm{c}$ nuclear magnetic resonance spectroscopy assessment in offspring of type 2 diabetic parents. Diabetes. 1999;48:1600-1606

33. Schrauwen-Hinderling VB, Kooi ME, Hesselink MK, Jeneson JA, Backes WH, van Echteld CJ, van Engelshoven JM, Mensink M, Schrauwen P. Impaired in vivo mitochondrial function but similar intramyocellular lipid content in patients with type 2 diabetes mellitus and bmimatched control subjects. Diabetologia. 2007;50:113-120

34. Hoeks J, Hesselink MK, Russell AP, Mensink M, Saris WH, Mensink RP, Schrauwen P. Peroxisome proliferator-activated receptor-gamma coactivator-1 and insulin resistance: Acute effect of fatty acids. Diabetologia. 2006;49:2419-2426

35. Phielix E, Meex R, Ouwens DM, Sparks L, Hoeks J, Schaart G, Moonen-Kornips E, Hesselink $M K$, Schrauwen P. High oxidative capacity due to chronic exercise training attenuates lipidinduced insulin resistance. Diabetes. 2012;61:2472-2478

36. Brands M, Hoeks J, Sauerwein HP, Ackermans MT, Ouwens M, Lammers NM, van der Plas MN, Schrauwen P, Groen AK, Serlie MJ. Short-term increase of plasma free fatty acids does not interfere with intrinsic mitochondrial function in healthy young men. Metabolism. 2011;60:1398-1405

37. Jans A, van Hees AM, Gjelstad IM, Sparks LM, Tierney AC, Riserus U, Drevon CA, Schrauwen $\mathrm{P}$, Roche HM, Blaak EE. Impact of dietary fat quantity and quality on skeletal muscle fatty acid metabolism in subjects with the metabolic syndrome. Metabolism.61:1554-1565

38. Samocha-Bonet D, Campbell LV, Mori TA, Croft KD, Greenfield JR, Turner N, Heilbronn LK. Overfeeding reduces insulin sensitivity and increases oxidative stress, without altering markers of mitochondrial content and function in humans. PLoS One. 2012;7:e36320

39. Brons C, Jensen CB, Storgaard H, Hiscock NJ, White A, Appel JS, Jacobsen S, Nilsson E, Larsen $\mathrm{CM}$, Astrup A, Quistorff B, Vaag A. Impact of short-term high-fat feeding on glucose and insulin metabolism in young healthy men. J Physiol. 2009;587:2387-2397 
40. Scheuermann-Freestone M, Madsen PL, Manners D, Blamire AM, Buckingham RE, Styles P, Radda GK, Neubauer S, Clarke K. Abnormal cardiac and skeletal muscle energy metabolism in patients with type 2 diabetes. Circulation. 2003;107:3040-3046

41. Diamant M, Lamb HJ, Groeneveld Y, Endert EL, Smit JW, Bax JJ, Romijn JA, de Roos A, Radder JK. Diastolic dysfunction is associated with altered myocardial metabolism in asymptomatic normotensive patients with well-controlled type 2 diabetes mellitus. J Am Coll Cardiol. 2003;42:328-335

42. Nakae I, Mitsunami K, Yoshino T, Omura T, Tsutamoto T, Matsumoto T, Morikawa S, Inubushi T, Horie M. Clinical features of myocardial triglyceride in different types of cardiomyopathy assessed by proton magnetic resonance spectroscopy: Comparison with myocardial creatine. J Card Fail. 2010;16:812-822

43. Krssak M, Winhofer Y, Gobl C, Bischof M, Reiter G, Kautzky-Willer A, Luger A, Krebs M, Anderwald $C$. Insulin resistance is not associated with myocardial steatosis in women. Diabetologia. 2011;54:1871-1878

44. van der Meer RW, Rijzewijk LJ, de Jong HW, Lamb HJ, Lubberink M, Romijn JA, Bax JJ, de Roos A, Kamp O, Paulus WJ, Heine RJ, Lammertsma AA, Smit JW, Diamant M. Pioglitazone improves cardiac function and alters myocardial substrate metabolism without affecting cardiac triglyceride accumulation and high-energy phosphate metabolism in patients with well-controlled type 2 diabetes mellitus. Circulation. 2009;119:2069-2077

45. Neubauer S, Horn M, Cramer M, Harre K, Newell JB, Peters W, Pabst T, ErtI G, Hahn D, Ingwall JS, Kochsiek K. Myocardial phosphocreatine-to-atp ratio is a predictor of mortality in patients with dilated cardiomyopathy. Circulation. 1997;96:2190-2196 


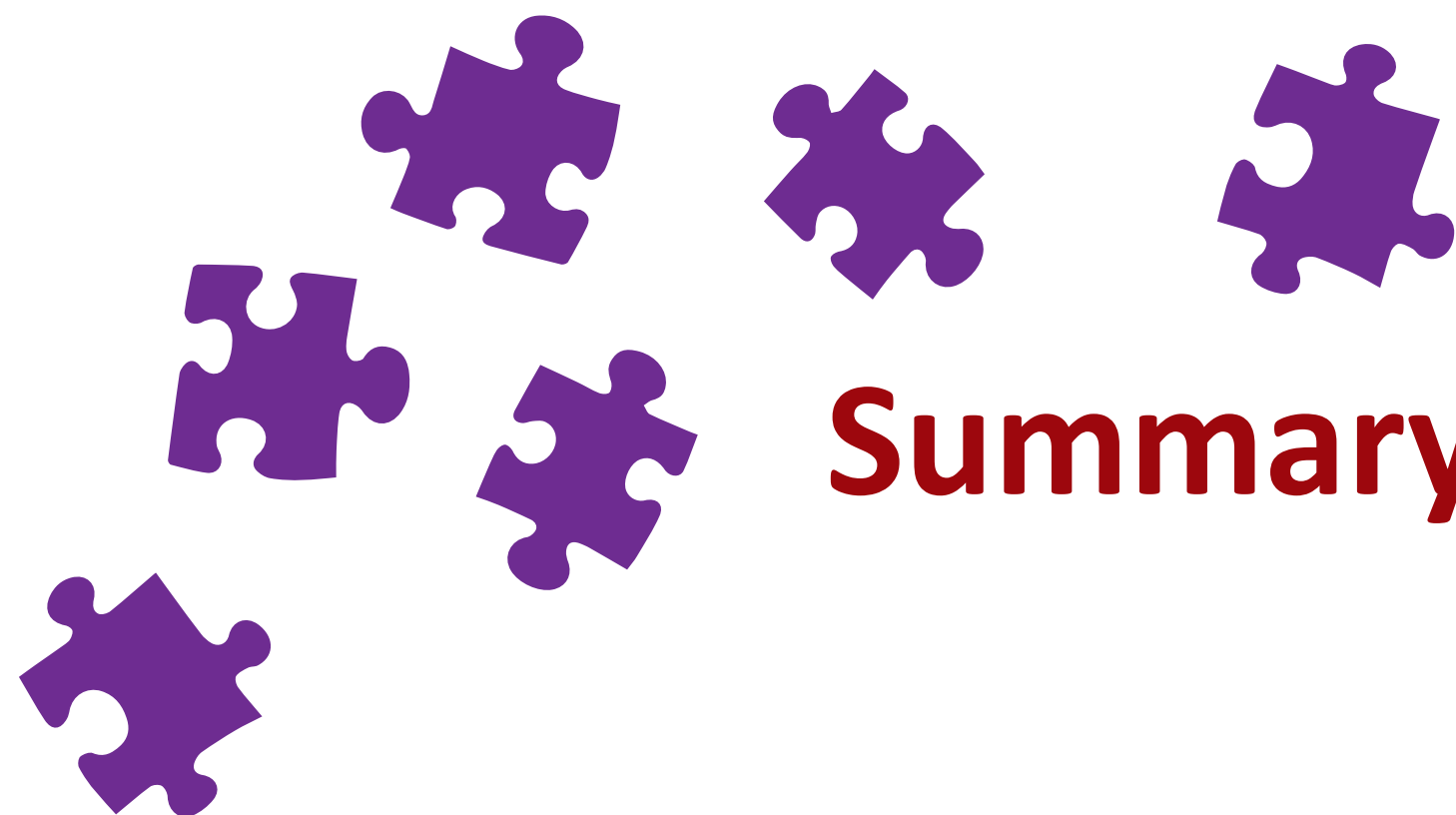

\section{Summary}

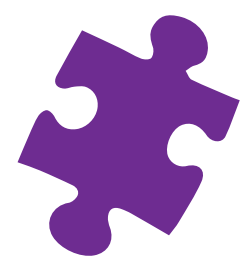

$\mathrm{CH}_{2}$

$\mathrm{CH}_{1}$ 


\section{Summary}

Type 2 diabetes is a growing problem with a prevalence of about $2.8 \%$ in 2000 which has been estimated to rise towards $4.4 \%$ in 2030 . We know that obesity is associated with the development of type 2 diabetes, but we only are beginning to understand why. While, in the case of overnutrition, the surplus of fat ingested is mostly stored in adipose tissue, part of it is also shuttled towards the other organs, amongst which skeletal muscle, liver and heart. If supply increases without increased energy demand, fat is stored rather than being oxidized with a low rate of lipid turnover as the resultant. This low turnover of this so-called ectopic fat has been associated with the development of insulin resistance, which is central to the pathology of type 2 diabetes. Ectopic fat has not only been associated with insulin resistance, but also with other type 2 diabetes related co-morbidities, like pancreatic failure, non-alcoholic fatty liver disease and cardiac problems (also known as diabetic cardiomyopathy). The detrimental effects of ectopic fat accumulation in type 2 diabetes are suggested to be mediated by an increased production of lipid intermediates, which have been shown to interfere with the insulin signalling cascade and induce cellular damage. A better understanding of these toxic mechanisms might help to better prevent and treat type 2 diabetes and its co-morbidities. Therefore this thesis focusses on the relationship between ectopic fat accumulation and the development of insulin resistance and diabetic cardiomyopathy.

In chapter $\mathbf{2}$ recent literature on the possible mechanisms involved in cardiac lipotoxicity is reviewed; we focused on the mechanisms related to cardiac lipid accumulation, which can cause cardiac problems in type 2 diabetes without the development of ischemia and elevated blood pressure. It becomes evident from literature that cardiac metabolism is disturbed under these conditions. Not only has the heart become more dependent upon fatty acids and fatty acid supply, but also cardiac metabolism has become less efficient (due to both an increased fat oxidation and uncoupling; both these mechanisms reduce the ATP/O2 ratio). Furthermore, although evidence in humans is scarce, it seem that cardiac mitochondrial oxidative capacity is lower in type 2 diabetes. Whether this is a cause rather than a consequence of cardiac lipid accumulation remains unclear. The decrease in cardiac mitochondrial capacity seems to be associated with an increased reactive oxygen species (ROS) production, which can peroxidize lipids and can cause cellular damage, aggravating cardiac malfunction in type 2 diabetes.

Animal studies have shown that certain lipids can damage the heart directly, either by inducing apoptosis (this was shown for palmitate), or via lipid intermediates that can interfere with the insulin-signalling cascade (DAGs and ceramides). Although there is a vast 
body of evidence in animals, there is little research done in this field in humans. Hence it remains to be elucidated whether these lipotoxic events, as witnessed in animals, are also applicable in humans with type 2 diabetic cardiomyopathy. In chapter $\mathbf{4 , 5}$ and $\mathbf{6}$ we have investigated Adipose Triglyceride Lipase deficient (ATGL-/-) mice and patients that are characterized by elevated ectopic lipid storage.

In order to be able to address this issue in mice, we first needed to develop a quick and reliable method for measuring cardiac function repeatedly in small rodents. In chapter 3 we have assessed different geometrical models for the calculation of ejection fraction (a measure of systolic cardiac function) in mice taking advantage of non-invasive MRI technology. We showed that the modified Simpson Rule model was the best and most accurate model to predict cardiac function measured with the gold standard (full volume imaging of the left ventricle).

To investigate the effects of neutral lipid storage in the heart and how this impacts mitochondrial function, we investigated ATGL-/- mice. ATGL-/- mice lack ATGL, which is the main enzyme responsible for the breakdown of neutral lipids stored in the cell (triacylglycerols; TAG). In these animals, fat can be stored, but cannot be degraded resulting in excessive fat storage. ATGL-/- mice develop cardiac failure and die at a young age (between 16-22 weeks). When we examined these mice in chapter 4 , we found that the extreme lipid accumulation in the heart was paralleled by a decreased cardiac function, increased fibrosis and decreased mitochondrial function. With progressive in cardiac fat accumulation with age, cardiac and mitochondrial function progressively deteriorates. Interestingly, also mRNA levels of PPAR- $\alpha$ and PPAR- $\delta$ target genes were down-regulated. These findings might indicate that lipolysis of cellular triglycerides by ATGL generates essential mediators involved in the generation of lipid ligands for PPAR activation, which ultimately drives oxidative metabolism.

In contrast, in chapter 5 in vivo PCr-recovery measurements with ${ }^{31} \mathrm{P}-\mathrm{MRS}$ in skeletal muscle of these animals did not reveal a defect in mitochondrial oxidative capacity. Therefore, the overall muscular mitochondrial oxidative capacity was comparable between the ATGL/- mice and healthy animals. Still the muscles from ATGL-/- mice displayed a significantly lower force production, higher glycogen depletion and increased muscle relaxation time compared to the healthy mice.

When we tried to restore PPAR-gene expression by pharmacological treatment of ATGL-/mice with PPAR- $\alpha$ agonists, the mitochondrial defects were completely reversed, cardiac function was restored and premature death was prevented in these animals. These data underline the importance of lipid droplet dynamics, as apparently fatty acids as ligands 
for PPAR mediated gene-expression need to be altered and liberated from the neutral lipid stores by ATGL. Furthermore, these findings also reveal a potential treatment for the excessive cardiac lipid accumulation and often-lethal cardiomyopathy in people with defects in the gene PNPLA2 encoding ATGL, causing neutral lipid storage disease (NLSDM). We sought to investigate in chapter 6 whether these mechanisms observed in the ATGL-/- mice might be similar in patients with NLSDM.

Neutral lipid storage disease with myopathy (NLSDM) is a rare genetic disorder due to a mutation in the gene encoding for adipose triglyceride lipase (ATGL). Patients exhibit (similar to the ATGL-/- mice) excessive ectopic lipid accumulation, leading to insulin resistance, myopathy, cardiac failure and premature death. We treated a NLSDM patient for 28 weeks with Bezafibrate (a PPAR-agonist). We also treated her sister, who carries a heterozygous mutation of the PNPLA2 gene and although she is not classified as a patient with NLSDM (as she only has one gene defect), she does present with an increased lipid storage and myopathy. Similar to the ATGL-/- mice, both subjects showed a marked increase in whole body fat oxidative capacity and skeletal muscle fat oxidative capacity after bezafibrate treatment, normalising oxidative metabolism to values found in gender-, age- and BMImatched controls. Parallel to these changes, ectopic fat accumulation was reduced after treatment, but still elevated compared to controls. Also a mild increase in metabolic clearance rate of glucose was found. Although leg strength (as measured by an extension test) seemed to consistently improve upon treatment, neither the hand-grip strength nor the elevated serum creatine kinase levels (marker for muscle damage) seemed to change. Cardiac function also remained impaired over the treatment period. Thus although we did find that the PPAR-treatment could normalise oxidative metabolism and reduce fat accumulation, it did not result in convincing clinical changes over this treatment period.

In the following part of the thesis, we wanted to focus more on type 2 diabetes and the role of lipid accumulation and mitochondrial function in diabetic cardiomyopathy. In chapter 7 we assessed the inter-relationships between fat accumulation, mitochondrial function, insulin resistance and metabolic flexibility (the capacity to switch between glucose and fat as a substrate for oxidation). We assessed this with simple and linear regression analysis in a relatively large group of type 2 diabetic patients and obese subjects. Although fasting plasma free fatty acid levels were significantly elevated in type 2 diabetic patients and mitochondrial function (measured with ${ }^{31} \mathrm{P}-\mathrm{MRS}$ ) was impaired, we did not find differences in skeletal muscle lipid accumulation. After simple and linear regression analysis over the whole group, we could not find a relationship between insulin resistance and metabolic flexibility nor with lipid accumulation. Mitochondrial function, however, appeared to be the sole predictor 
of basal Respiratory Exchange Ratio (RER), whereas whole-body glucose disposal (WGD) predicted both insulin-stimulated RER and metabolic flexibility. Also plasma free fatty acids seemed to contribute to insulin resistance as it correlated with both, whole body glucose disposal and metabolic flexibility. These results indicate that defects in skeletal muscle in vivo mitochondrial function in type 2 diabetic patients are only reflected in basal substrate oxidation and highlight the importance of glucose disposal rates and fatty acid supply as a determinant of substrate utilization in response to insulin. In the last two chapters we have focussed on whether manipulation of the fatty acid supply may affect fat storage and alter oxidative metabolism and how this affects insulin resistance and cardiac function.

In chapter $\mathbf{8}$ we sought to determine whether elevated plasma fatty acid concentrations could lead to cardiac lipid accumulation. Furthermore, we were interested whether an increase in fat accumulation in the heart, under physiological circumstances, might deteriorate cardiac energy status and cardiac function. This was established by an exercise protocol in young healthy volunteers. During exercise in the fasting condition, plasma free fatty acids will rise, whereas glucose supplementation blunts this response. Indeed, plasma free fatty acid concentrations were increased threefold during exercise and nine-fold during recovery in the fasted state compared with the glucose-fed state. This elevation in plasma free fatty acids resulted in an increase in cardiac lipid content in the fasted state. Although cardiac energy status (PCr/ATP ratio, measured with ${ }^{31} \mathrm{P}-\mathrm{MRS}$ ) decreased in the fasted state compared to the glucose-supplemented state, the ejection fraction post-exercise was higher in this group. Hence, elevated plasma free fatty acid concentrations during and after exercise, led to increased cardiac lipid content, but did not acutely hamper systolic function.

In chapter $\mathbf{9}$ we tried to lower fatty acids, in order to assess whether lowered ectopic fat accumulation might ameliorate cardiac function and insulin sensitivity in type 2 diabetes. Here, we used the nicotinic acid analogue 'Acipimox', known for its profound and fast lowering of plasma free fatty acids, by inhibition of lipolysis in adipose tissue. Although some studies have applied this medication with success with dosages of $250 \mathrm{mg} 4 \mathrm{dd}$ every 6 hours, in our study a dosage of 250mg 3dd after every meal, led to a rebound effect. Instead of lowering the plasma free fatty acids, plasma free fatty acids became significantly elevated. This led to an increase in insulin resistance and a tendency to an increased skeletal and cardiac muscle lipid content after 2 weeks of treatment. However, hepatic lipid content remained unchanged. In vivo skeletal muscle mitochondrial function decreased, whereas cardiac energy metabolism did not change. Diastolic and systolic cardiac function tended to decrease. The study indicates that moderate elevation of plasma fatty acids may indeed 
be responsible for the increased lipid accumulation in skeletal muscle seen in obesity. Furthermore, an increased lipid accumulation and supply tend to decrease oxidative metabolism in skeletal muscle, but not in the heart.

Overall the results obtained in this thesis point out that lipid accumulation per se does not need to be detrimental for neither skeletal muscle nor heart. Furthermore, our findings underline the importance of lipid droplet dynamics, as apparently lipids do not only serve as fuel but also can serve as ligands regulating PPAR-gene expression and oxidative metabolism which may be of vital importance for patients with neutral lipid storage disease (NLSDM). 



\section{Samenvatting}

Hoewel we weten dat overgewicht een risico vormt voor het ontwikkelen van type 2 diabetes, beginnen we nu pas te begrijpen waarom dat zo is. In het geval van het ontstaan van overgewicht wordt er meer energie gebruikt dan dat er verbrand wordt. Het overschot aan vetten uit de voeding wordt met name in het vetweefsel opgeslagen, echter een klein deel wordt ook in de organen opgeslagen (zoals de lever, de spieren, de alvleesklier en het hart). De opslag van vetten in de organen blijkt samen te gaan met de ontwikkeling van type 2 diabetes en de daarbij behorende co-morbiditeiten zoals niet-alcohol afhankelijke leververvetting, het falen van de pancreas en cardiovasculaire problemen (o.a. diabetische cardiomyopathie). Vetopslag in de organen kan interfereren met de insuline signaleringscascade en hierdoor bijdragen aan de ontwikkeling van insuline resistentie en type 2 diabetes. Er wordt ook gesuggereerd dat vetopslag in de organen in sommige gevallen schadelijk zijn voor een cel. We begrijpen echter nog niet goed wanneer vetopslag schadelijke gevolgen heeft en ook niet waarom dit gebeurt. Daarom is het doel van dit proefschrift te onderzoeken hoe vetstapeling bij mensen met overgewicht kan leiden tot type 2 diabetes en de daaraan gerelateerde cardiomyopathie.

In hoofdstuk 2 van dit proefschrift hebben we literatuur over diabetische cardiomyopathie op een rijtje gezet; het blijkt dat mensen met type 2 diabetes een verhoogde kans hebben op cardiomyopathie in vergelijking met mensen zonder diabetes met dezelfde lengte en hetzelfde gewicht, zelfs wanneer werd gecorrigeerd voor een verhoogde bloeddruk en coronairvaat-lijden.

Er zijn een aantal mechanismen die bij de ontwikkeling van cardiomyopathie een rol zouden kunnen spelen. Zo blijkt dat het hart bij mensen met type 2 diabetes veel afhankelijker is van de vetoxidatie voor het verkrijgen van energie. Aangezien er meer zuurstof nodig is voor vet- dan voor glucose oxidatie, wordt het hart hierdoor minder efficiënt (er is meer zuurstof nodig voor de productie van eenzelfde hoeveelheid energie). Daarnaast zijn er aanwijzingen dat de oxidatie van zowel glucose en vetten door de mitochondria minder goed verloopt. Hierdoor zou er dus eveneens minder energie beschikbaar zijn voor het hart. Door een verminderd functioneren van de mitochondria worden er ook meer zuurstofradicalen gevormd, welke op hun beurt schade kunnen veroorzaken aan de cel en mitochondria. Ook zijn er aanwijzingen dat bepaalde vetten zoals palmitaat direct kunnen leiden tot de initiatie van apoptosis (celdood) en kunnen onvolledig verbrandde vetten (DAGs en ceramiden) toxische effecten hebben. Echter, het meeste bewijs voor deze bevindingen komt uit dierstudies en moeten bevestigd worden bij mensen. 
In hoofdstuk 4,5 en 6 onderzochten we in een muismodel en in patiënten met een genetische afwijking, of neutrale vetstapeling schadelijk is voor het hart en waardoor dit eventueel veroorzaakt zou kunnen worden.

Alvorens wij de muizen konden bestuderen, hebben we in hoofdstuk 3 allereerst een techniek ontwikkeld om met MRI de hartfunctie bij knaagdieren snel en efficiënt te kunnen bepalen. Hieruit bleek dat de zogenaamde 'Modified Simpsons rule' model een zeer goed model is voor het kwalitatief en kwantitatief bepalen van de hartfunctie bij muizen en ratten. Deze techniek hebben we dan ook in de volgende hoofdstukken toegepast voor het berekenen van de hartfunctie.

Om te onderzoeken of de stapeling van vetten in neutrale vorm (triglyceriden) in het hart en de skeletspier ook toxische effecten teweeg kan brengen, hebben we ATGL-/- muizen onderzocht (hoofdstuk 4). ATGL codeert voor 'Adipose Triglyceride Lipase' dat de opgeslagen triglyceriden vrij maakt uit de neutrale lipiden opslag en deze omzet in diacylglycerol en vetzuren. Muizen met ATGL deficiëntie hebben dus wel de capaciteit om triglyceriden op te slaan, maar niet om deze af te breken. Deze muizen hebben dan ook tot 20 keer meer triglyceriden in het hart en tot wel 3 keer meer triglyceriden in de skeletspieren vergeleken met gezonde muizen. Ten gevolge hiervan ontwikkelden deze muizen allen cardiomyopathie op jonge leeftijd, waaraan ze vroegtijdig overleden. In ons onderzoek vonden we dat de cardiale mitochondria niet goed functioneerden en dat mRNA levels van PPAR- $\alpha$ en PPAR- $\delta$ gereguleerde genen verlaagd waren. Deze genen reguleren het oxidatieve metabolisme. Deze bevindingen zouden erop kunnen wijzen dat lipolyse van de opgeslagen triglyceriden door ATGL essentiële mediatoren genereerd welke betrokken zijn bij de vorming van vetzuren als liganden voor de activatie van de PPAR-receptoren. Deze laatste zijn betrokken bij de regulatie van het oxidatieve metabolisme.

In tegenstelling tot deze bovenstaande bevindingen konden we in hoofdstuk $\mathbf{5}$ met in vivo $\mathrm{PCr}$-recovery metingen met ${ }^{31} \mathrm{P}-\mathrm{MRS}$ in de skeletspieren van ATGL-/- muizen geen defecten aantonen in de mitochondriële oxidatieve capaciteit. De mitochondriële oxidatieve capaciteit in de skeletspier was vergelijkbaar tussen ATGL-/- muizen en gezonde dieren. Wel vonden we dat de spieren van deze ATGL-/- muizen significant minder kracht konden produceren en een sterkere mate van glycogeen depletie vertoonden tijdens inspanning.

Door synthetische PPAR-agonisten aan de ATGL-/- muizen te geven (in de vorm o.a. fibraten), normaliseerde de gen-expressie van de PPAR- $\alpha$ en PPAR- $\delta$ gereguleerde genen, normaliseerde de mitochondriële functie en cardiale functie en bleven deze muizen in leven. 
Deze bevindingen zijn zeer belangrijk, niet enkel omdat dit de theorie dat lipolyse via ATGL nodig is om lipide-liganden te creëren voor de activatie van de PPARs bevestigt, maar ook omdat er patiënten zijn met een vergelijkbaar gendefect. Deze patiënten zouden mogelijk profijt kunnen hebben van een behandeling met deze PPAR-agonisten.

In hoofdstuk 6 onderzochten we of patiënten met "NLSDM' (neutral lipid storage disease with myopathy) baat zouden kunnen hebben bij een behandeling met PPAR-agonisten. NLSDM wordt veroorzaakt wordt door een defect in het PNPLA2 gen, welke codeert voor het enzym ATGL. Net als ATGL deficiënte muizen worden deze patiënten gekenmerkt door grote hoeveelheden vetstapeling in de organen, waaronder in de skeletspieren, hart, lever en pancreas. Deze patiënten ontwikkelen ook cardiomyopathie waaraan zij vaak vroegtijdig overlijden. Tot op heden was er geen behandeling voor deze patiënten behoudens voedingsadviezen of behandeling van de symptomen. In hoofdstuk 6 behandelden we een patiënt en een drager met klachten (deze persoon heeft ook een verhoogde vetstapeling en last van spierzwakte, maar heeft enkel een heterozygoot gendefect). Bij behandeling van deze twee personen met Bezafibraat (een PPAR-agonist), nam de vetstapeling in de skeletspier en het hart af, verhoogde de vetoxidatieve capaciteit en verbeterde de mitochondriële functie. Ook leek de kracht van de bovenbeenspieren toe te nemen, echter dit ging niet samen met een verbetering van de handknijpkracht. Ook zagen we geen echte verbeteringen in de hartfunctie bijdeze twee personen. Ook al normaliseerde het metabolisme tot normaalwaarden vergeleken met leeftijd en BMI-gematchte proefpersonen, maar de spierkracht en cardiale functie bleven verminderd ten opzichte van de controle groep. Bovendien veranderde de hartfunctie niet tijdens deze studie. Dus alhoewel het metabolisme bij deze patiënten normaliseerde, zagen we (nog) geen significante verbeteringen in de klinische uitkomstmaten.

In de hierop volgende hoofdstukken wilden we ons meer toeleggen op de rol van de mitochondriële dysfunctie en ectopische vetstapeling bij het ontstaan van insuline resistentie bij type 2 diabetes. Daartoe onderzochten we een grote groep patiënten met en zonder type 2 diabetes, met als doel om vast te stellen of er verbanden te vinden zijn tussen mitochondriële dysfunctie, metabole flexibiliteit, vetstapeling en insuline resistentie.

Uit ons onderzoek bleek dat er geen verschil was in vetstapeling in de spieren tussen de type 2 diabeten en gezonde controles. Echter patiënten met type 2 diabetes hadden wel een verlaagde in vivo oxidatieve capaciteit (gemeten met $\left.{ }^{31} \mathrm{P}-\mathrm{MRS}\right)$. Deze mitochondriële dysfunctie correleerde met de basale Respiratory Exchange Ratio (RER), welke een reflectie is van de verhouding van vet/suiker verbranding. Het had echter geen effect op de insuline gestimuleerde RER, welke wel sterk correleerde met de insuline gestimuleerde glucose 
opname (maat voor insuline resistentie) en de vetzuurspiegels in het bloed. De metabole flexibiliteit (de capaciteit om tussen vetten en suikers als brandstof te wisselen na insuline stimulering) werd dan ook met name door de insuline gemedieerde 'glucose disposal rates' bepaald. Kortom in rust zijn met name de mitochondria de bepalende factor voor de verbranding van suikers en vetten, terwijl tijdens insuline stimulatie met name de suiker opname capaciteit/ernst van insuline resistentie en vetzuurspiegels van belang zijn.

In hoofdstuk 8 en $\mathbf{9}$ onderzochten we of het verhogen of verlagen van de vrije vetzuur spiegels in het bloed de ectopische vetstapeling zou kunnen verlagen en of dit gunstig zou kunnen zijn voor de cardiale functie en insuline gevoeligheid. Hiertoe onderzochten we in hoofdstuk 8 een groep gezonde jonge mannen, welke we twee uur gevast lieten fietsen. Deze inspanning in gevaste toestand zet het vetweefsel aan tot het vrij maken van vetzuren voor verbranding en leidt dus tot een verhoging van de vrije vetzuren in het bloed. We zagen dat de verhoging van de vetzuurspiegels leidde tot een verhoging van de vetstapeling in het hart, gemeten met ${ }^{1} \mathrm{H}-\mathrm{MRS}$. Dit ging gepaard met een verlaging van de cardiale energie status (gemeten met ${ }^{31} \mathrm{P}-\mathrm{MRS}$ ). Ondanks een verhoging van de vetstapeling en een verlaging van de energiestatus bleek de functie van het hart te verbeteren. Het onderdrukken van de stijging in vetzuren, door de consumptie van glucose tijdens het fietsen, voorkwam vetstapeling in het hart en leidde ook tot het behouden van de energiestatus van het hart.

In hoofdstuk 9 onderzochten we of het geneesmiddel Acipimox gedurende twee weken de vrije vetzuren kan onderdrukken in patiënten met type 2 diabetes en of dit mogelijk een verlaging van de vetstapeling in de organen en een verbetering van de insuline gevoeligheid en cardiale functie tot gevolg had. Acipimox wordt gegeven voor de behandeling van een verhoogd cholesterol, maar het staat ook bekend om zijn sterke en acute effect op de verlaging van de vrije vetzuren in het bloed. Echter tegen onze verwachtingen in leidde een behandeling met Acipimox gedurende 2 weken tot een verhoging in plaats van een verlaging van de vrije vetzuren, terwijl het cholesterol werd verlaagd. Deze verhoging van de vrije vetzuren ging gepaard met een verhoogde vetstapeling in de skeletspier en een verlaging van de insuline gevoeligheid. Ook de cardiale functie leek enigszins achteruit te gaan. Daarentegen veranderde het vetgehalte van het hart en de energie status van het hart niet. Ook het vetgehalte van de lever veranderde niet. De verbrandingscapaciteit van de mitochondria van de skelet spieren in vivo gemeten met $\mathrm{PCr}$-recovery rates met ${ }^{31} \mathrm{P}-\mathrm{MRS}$, leek wel enigszins achteruit te gaan. De ex vivo mitochondriële functie daarentegen nam toe i.p.v. af; dit zou erop kunnen duiden dat nicotinezuur analogen wellicht een direct effect hebben op de mitochondriële functie, maar dat deze in vivo niet tot hen recht komen door 
de verhoogde vetzuren in het bloed en vetstapeling in de spieren. Desondanks lijkt erop te duiden dat een verlaging van de insuline sensitiviteit na behandeling met Acipimox, mogelijk gemedieerd wordt door een verhoging van de vetstapeling in de skelet spier en een verlaging van de mitochondriële functie aldaar.

Samenvattend lijken de resultaten van deze studies erop te wijzen dat ectopische vetstapeling in de organen niet a priori schadelijk hoeft te zijn. De vetstapeling in de organen heeft een belangrijke functie in de regulering van cellulaire processen en speelt een belangrijke rol bij patiënten met NLSDM. 


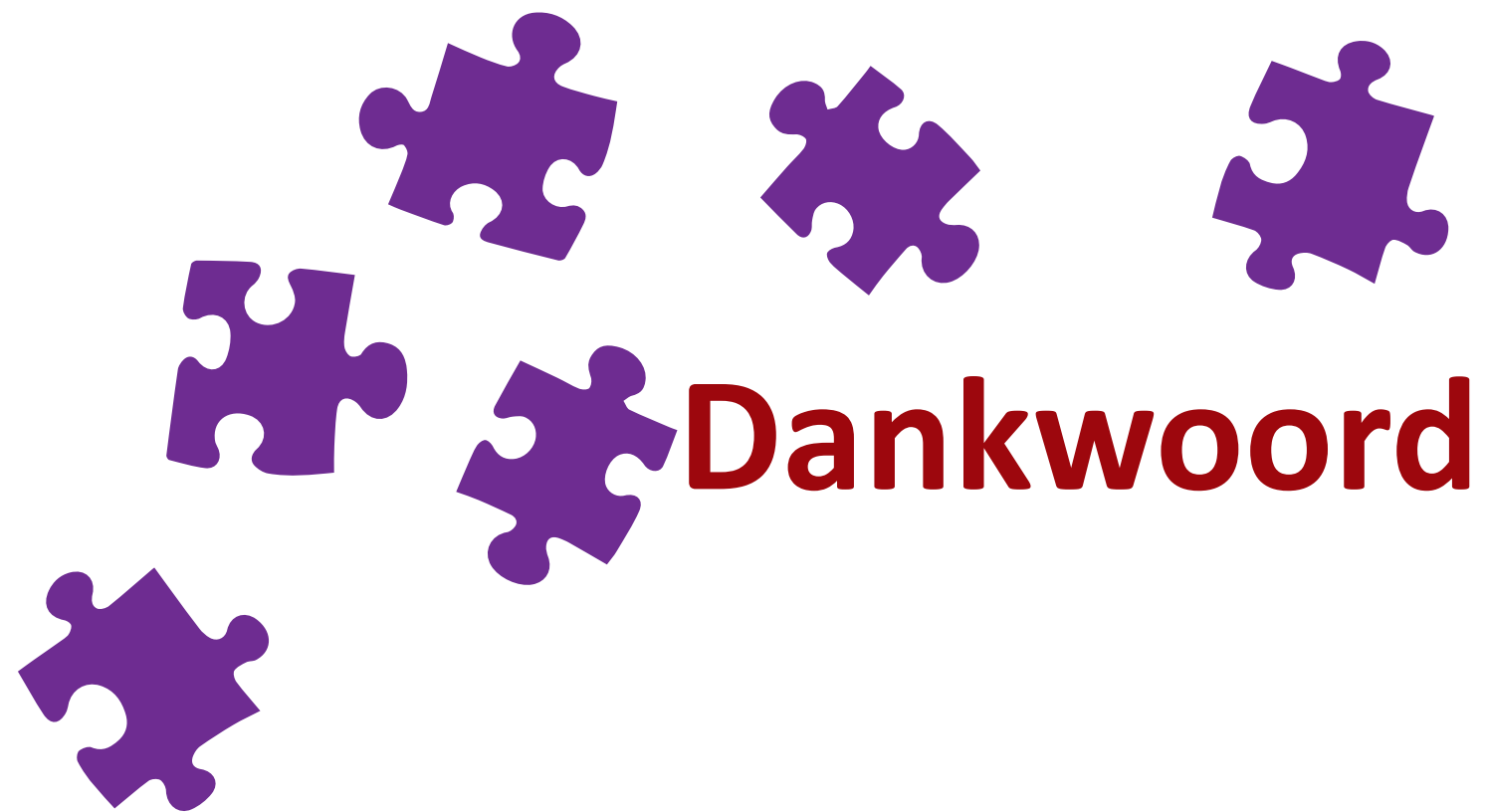

$\mathrm{CH}_{2}$

$M \mathrm{CH}^{\mathrm{CH}_{3}}$ 
Eindelijk is het dan zo ver; het boekje is af en het promotie traject is afgerond. Ongelofelijk! Niet dat ik er ooit aan getwijfeld heb dat het zou gaan gebeuren, maar het is zo snel gegaan allemaal dat ik nauwelijks kan geloven dat het echt al zo ver is. Natuurlijk heb ik veel te danken aan de hulp, inzet, enthousiasme en volharding van anderen. Daarnaast is het een fantastische tijd geweest waarvoor ik iedereen van harte wil bedanken! Ik ga mijn best doen om niemand te vergeten, maar 'errare humanum est!' (Dit slaat natuurlijk ook terug op de vele spellingsfouten die waarschijnlijk nog in dit boekje zullen staan, en ja de spellingschecker staat aan).

Ten eerste gaat mijn dank uit aan alle mensen die deelgenomen hebben aan het onderzoek. Zonder jullie was niets van dit alles mogelijk geweest. De wetenschap vaart op gemotiveerde en geïnspireerde mensen zoals jullie; het was dan ook geweldig om met jullie samengewerkt te hebben en jullie verhalen te hebben mogen delen. Men zegt wel eens 'de wetenschap dient de mens' maar zonder mensen die de wetenschap willen dienen, komen we helaas nergens. Ik heb dan ook bewondering voor de inzet en volharding die vele van jullie toonden en de manier waarop jullie vaak jullie eigen belang achterstelden op dat van het grote gemene goed. Bedankt voor de talloze spierbiopten die ik heb af mogen nemen, de uren die jullie op bed en in de scanner doorgebracht hebben en de tijd die jullie aan het onderzoek geschonken hebben. Het was een mooie ervaring voor mij en een mooie toevoeging aan de wetenschap. Ik hoop dat het jullie allen goed gaat en dat ik jullie nog eens in de wandelgangen tegen mag komen.

Ten tweede gaat mijn dank uit aan de leden van de leescommissie. Prof. Mensink, beste Ronald, bedankt dat jij de voorzitter van de leescommissie wilde zijn. Ik ben zeer vereerd dat jij deze zware taak tot je hebt genomen. Ondanks dat mijn thesis waarschijnlijk minder makkelijk leest dan de 'Donald-Duck', hoop ik dat je de inhoud toch hebt weten te waarderen. Daarnaast wil ik je ook persoonlijk bedanken voor de fijne jaren die ik met je heb mogen werken. Je was een fijne, vriendelijke en zeer begane vakgroep voorzitter. Daarnaast was je ook een zeer betrokken collega die altijd de tijd nam om een babbeltje met iedereen te maken. Ook kan je altijd om een goede grap lachen. Ik hoop dan ook van harte voor je dat de Universiteit snel het concept van 'uniseks-toiletten' zal introduceren zodat het leven voor de gewone man op niveau 0 weer enigszins leefbaar zal worden.

Prof. De Haan, beste Michiel; ik voelde me ook zeer vereerd dat jij als Professor en opleider in de Radiologie deel wilde nemen aan de commissie. Natuurlijk is dit metabole onderzoek (nog) niet echt de standaard in de radiologie, maar ik hoop dat je de veelzijdigheid van de beeldvorming binnen het metabole onderzoek kan waarderen. Daarnaast wil ik je ook 
persoonlijk bedanken voor het feit dat je me (samen met de andere leden van de sollicitatie commissie) een kans hebt gegeven om te laten zien wat ik kan en vertrouwen in mij hebt als beginnende AIO. Ik hoop dan ook alle verwachting waar te maken en binnen een paar jaar een goede radioloog te worden!

Beste Prof. de Windt, beste Leon, ondanks dat we nog niet echt de kans hebben gekregen om van gedachten te wisselen, hebben we elkaar wel al vaak gezien op bijeenkomsten, o.a. van het CTMM. Ik was altijd onder de indruk van de kwaliteit en diepgang van het onderzoek wat jullie binnen de cardiologie uitvoeren. Hoewel ons onderzoek veel raakvlakken heeft, zijn er ook grote verschillen in de benadering en uitvoering van het onderzoek. Ik hoop dan ook dat u met veel plezier ook ons onderzoek hebt gelezen en ben zeer verheugd dat $u$ in de commissie plaats wilde nemen. Hopelijk kunnen we in de toekomst zowel in de kliniek als in het onderzoek eens van gedachten wisselen over enkele onderwerpen.

Beste Dr. Jazet, beste Ingrid, bedankt voor uw deelname aan de leescommissie van mijn thesis. Zoals u reeds aangaf is het afronden van een promotietraject een bijzondere mijlpaal. Ik ben dan ook zeer blij dat $u$ daar deel van uit maakt, daar u zelf veel elegante interventie studies met patiënten met type 2 diabetes heeft uitgevoerd. Ik heb uw onderzoeken altijd met veel plezier gelezen en zal dit natuurlijk ook blijven doen! Bedankt voor uw bijdrage aan de leescommisie. Dear Dr. Patricia lozzo, thank you very much for your participation in the PhD thesis committee. I have always admired your research which has much common ground compared with our research presented here in this thesis. Therefore it is an honour to have you on our thesis defence committee and I am really looking forward to meeting you.

Dan gaat mijn dank natuurlijk ook uit naar mijn Promotoren Prof. Schrauwen en Prof. Hesselink, en mijn co-promotor Dr. Schrauwen-Hinderling.

Beste Prof. Schrauwen, beste Patrick, bedankt voor het vertrouwen dat je altijd in me hebt gehad. Ik kwam groen en naïef bij jullie solliciteren nadat ik dacht dat ik het licht had gezien na een aantal weken moleculair onderzoek tijdens mijn WESP stage in Parijs. Hoewel ik nooit spijt gehad van mijn keuze, was het heel anders dan dat ik me in den beginne had voorgesteld. Zo dacht ik dat ik dagen en nachten op het lab zou staan, maar eigenlijk heb ik het lab in de afgelopen 4 jaar nauwelijks gezien (je bent dus niet de enige die de weg op het lab niet weet;)). Ik heb daarentegen wel de kans gekregen om veel andere mooie nieuwe technieken te leren. Ook werd het mogelijk gemaakt om zowel humane studies als dier studies te doen wat uiteindelijk resulteerde in zeer mooi translationeel onderzoek. Ik had 4 jaar geleden niet durven dromen dat ik zo'n mooie thesis zou mogen afronden. Maar doordat jij (en Matthijs) zo'n mooie, brede en bedreven onderzoeksgroep hebben weten op 
te bouwen is letterlijk de 'sky de limit'. Ik ben ongelofelijk blij dat ik daar een deel van uit heb mogen maken en een steentje bij heb mogen dragen. Ik hoop dat ik in de toekomst nog vaak met je samen kan werken vanuit de kliniek in projecten die MUMC+ overstijgend zijn. Daarnaast wil ik je ook bedanken voor de leuke tijd die ik de afgelopen 4 jaar en 4 maanden heb gehad (eigenlijk 4 jaar als je het zwangerschapsverlof eraf trekt). Dat komt o.a. door jouw positieve instelling als begeleider waarbij je af en toe ook veel geduld moest uitoefenen met mij als AIO. Bedankt voor je geduld met de vele revisies die je van mij toegestuurd kreeg en voor je geduld met de vele taalfouten die veelal in deze verschillende versies in verschillende vormen te vinden waren. Bedankt voor je durf en doorzettingsvermogen waarmee je dingen hebt verwerkelijkt waar ik (en anderen) soms de hoop al opgegeven had. Bedankt voor je snelle en adequate reacties; ik denk dat er weinig begeleiders/promotoren zijn die zo snel en efficiënt papers reviewen en op e-mailtjes antwoorden, ongeacht de drukte; dat is echt bewonderenswaardig. Bedankt voor je goede gevoel voor humor, die het leven soms makkelijker maakten, ook als dit soms wellicht een beetje ver ging (iets met batterijen en een computer?). Bedankt voor je 'enthousiasme' in plaats van 'frustratie' rondom mijn zwangerschap. Ik hoop dan ook dat je nog veel mooie dingen mag doen in de wetenschap, maar vooral ook dat het jou, Vera en jullie gezinnetje altijd goed mag gaan!

Beste Prof. Hesselink, beste Matthijs, het oprechte enthousiasme dat je altijd uitstraalt over de onderwerpen die wij (al dan niet) onderzoeken, werkt aanstekelijk. Je kennis van zaken doet me dan ook altijd verbazen, omdat je altijd en van alles wel iets weet wat aanleiding geeft tot leuke en verhelderende discussies. Ik heb dan ook bewondering voor je kennis en je enthousiasme en hoop dan ook dat je nog lang kan doen wat je leuk vindt, naast het (sleutelen aan) fietsen. Ook je communicatieve en sociale vaardigheden hebben ertoe geleid dat je mooie dingen zoals de Nature paper voor elkaar wist te krijgen. Echt super bedankt voor de geweldige 4 jaar die we samen hebben mogen werken, en ik hoop dan ook van harte dat alles goed met gaat, zowel wetenschappelijk als persoonlijk als met je gezin.

Beste Dr. Schrauwen-Hinderling, beste Vera, ik heb vanaf het begin ontzettend veel bewondering voor je gehad en ben dan ook echt zeer verheugd dat jij (meer dan terecht) mijn co-Promotor bent! Aan de andere kant ook wel jammer, anders had ik je wellicht paranimf kunnen maken, maar dit is nog beter! Je enthausiasme, inzet en eindeloze hartelijkheid is echt een voorbeeld voor iedereen. Al op het sollicitatie gesprek bracht jij rust en vertrouwen. Dat jij dan ook mijn eerste kamergenootje werd was voor mij een feest! Het kenmerkt je dan ook, dat je de eerste 2 weken op ons kamertje aanwezig was terwijl je eigenlijk maar 2 dagen bij de humane biologie werkte. Dan heb ik het nog niet gehad over de vele repen 
chocolade die je altijd vanuit Zwisterland mee bracht als lekkernij bij de koffie. Je bent een uitzonderlijke onderzoeker die in korte tijd de Nederlandse taal beheerste en vanuit een biologische studie op korte termijn de fysica van MRS onder de knie kreeg. Bovendien kan je je kennis ook bijzonder goed over brengen en heb je me de kneepjes van het vak weten te leren. Je bent dan ook met Eline K. het brein en de drijvende kracht achter de spectroclub. Dit is een fantastisch initiatief waarvan ik hoop dat het nog lang zal blijven bestaan en hopelijk ook de kliniek ten goede zal komen in de toekomst. Ondanks dat je zelf 3 kinderen hebt, ben je dag en nacht bereikbaar voor problemen en altijd bereid zelf de handen uit de mouwen te steken. Ontzetten veel respect voor de manier waarop jij al deze dingen managed (samen met Patrick natuurlijk). Bedankt voor je inzet en vertrouwen in mij en voor de 4 jaar. Bedankt dat je samen met Eline K. me geholpen hebt om ook mijn carrière verder uit te bouwen. Ook ontzettend bedankt voor de persoon die je bent, de persoon die altijd tijd voor mensen neemt, ambitieus en bescheiden is (een uitzonderlijke combinatie), altijd vriendelijk en vrolijk. Bedankt voor de 4 fijne jaren die wij als collegae hebben doorgebracht. Spectro-4-ever!

Natuurlijk wil ik ook Dr. Eline Kooi bedanken. Ondanks dat je helaas niet officieel in mijn promotie team zit, maak je er weldegelijk deel van uit. Heel erg bedankt voor de tijd die je in me gestoken hebt en voor het helpen bij het verder helpen van mijn carrière. Natuurlijk zijn we eigenlijk meer collega's geworden nu ik ook bij de radiologie aangesteld ben. Ik hoop dan ook dat we in de toekomst nog vaak samen zullen werken.

Dan wil ik graag Dr. Havekes bedanken. Beste Bas: alhoewel je pas halverwege mijn promotietraject begon bij als Endocrinoloog bij de Interne Geneeskunde van het MUMC, heb je een grote bijdrage geleverd aan mijn promotie traject. We hebben samen ongelofelijk veel tijd en energie gestoken in hoofdstuk 6, wat gelukkig ook resulteerde in een mooie paper. Daarnaast was je altijd bereikbaar voor vragen en problemen van klinische aard voor alle lopende humane studies. Je was een grote steun en een zeer fijne vertrouwenspersoon met een ongelofelijke wetenschappelijke en klinische kennis. Bedankt voor onze prettige samenwerking, ik hoop dat onze paden in de toekomst nog vaak zullen kruisen.

Daarnaast gaat mijn dank natuurlijk uit naar al mijn collega's van de SHOCk (voor de buitestaanders: Schrauwen-Hesselink-Onderzoeks-Combinatie). Ten eerste natuurlijk mijn paranimf Lena; toen ik net begon als AIO, werkte jij reeds als 'exchange student' bij Vera. Het klikte meteen. Ik ben dan ook erg blij dat Patrick en Vera veel vertrouwen in je hadden en je een PhD aanboden. Dat je de stap hebt durven zetten om vanuit Noorwegen definitief 
naar hier te komen, voor de liefde van je leven, ver weg van vrienden en familie, zegt veel over je durf en volharding. Als je ergens voor gaat, dan ga je ook voor de volle $100 \%$.

Deze beslissing maakte het mogelijk dat we op veel projecten samen konden werken. Ook naast het werk hebben we altijd veel plezier gehad samen. Ik ben ook blij dat jij degene was die ik kon toevertrouwen dat ik zwanger was. Ik wil je dan ook bedanken voor alle uren die je daardoor extra in onze studies hebt moeten steken. Nooit waren de uren te vroeg of te laat, de uren die ik met jou samengewerkt heb, waren geweldig. Takk!

Lauren, you were an amazing roommate; I never had a better roommate than you! Although you have some strange eating habits (no comments), the craziness of sharing a room with you made it all worth-while. Thank you for letting me into your life. I really hope everything is working out for you, Heather and Punkin and that you can make all your wildest dreams come true. Miss you a lot; have a diet coke on me, as I will have many on you!

Sabina we also worked together quite extensively. You shared all your animal 'secrets' with me, for which I am very thankful. Although my first impression of you was very quiet and shy girl, you surely showed me otherwise! You are a very driven and inspirational person. Also, you have particular taste for Belgian Beer and Belgian men. We had many good times together; I hope everything will work out for you in Canada and that you will come back to Belgium regularly, not only for Bart, but also for your old colleagues who miss you very much!

Silvie ondanks dat we maar korte tijd samengewerkt hebben, was het altijd erg gezellig! Jij staat altijd voor iedereen klaar en verzorgt altijd alles tot in de puntjes. Naast een fantastische onderzoeker ben je ook een geweldig persoon. Ik hoop dan ook dat je een mooie toekomst te gemoed gaat en nog veel plezier zal hebben in wat je doet en af en toe niet vergeet om ook aan jezelf te denken.

Esther $\mathbf{P}$, of hoe je jezelf ook wel graag noemt, EPs; Ik heb in Maastricht maar kort met je gewerkt en ben helaas maar heel kort mijn kamergenootje geweest. Toch heb ik gelukkig wel het genoegen gehad om een aantal projecten met jou uit te voeren in de tijd dat jij werkzaam was in Düsseldorf. Ondanks alle politieke spelletjes en communicatieve moeilijkheden, hebben wij het altijd goed met elkaar kunnen te vinden. Je bent een zeer gedreven en ambitieuze onderzoeker die als ze ergens haar zinnen op zet, ongelofelijk veel voor elkaar weet te krijgen. Ook op het werk en daarbuiten ben je zeer sociaal en altijd begaan met iedereen en aanwezig op alle activiteiten. Ik hoop dat je je ambities zult verwerkelijken en alles je goed zal gaan.

Bram; je bent een leuke, spontane collega die hard werkt, voor iedereen klaar staat, zeer openhartig is en altijd wel iets te vertellen heeft (ik jullie de details besparen). Ik heb 
ontzetten met je, maar ook om je kunnen lachen. Bedankt daarvoor. Ik hoop dat alles gaat lukken met je studies en je een mooi PhD project mag afronden.

Bianca je goedlachsheid maakt eenieders dag goed. Ik hoop dat je dat voor altijd weet te behouden. Zoals je weet gaat onderzoek soms met wat tegenslagen gepaard, maar jij weet je er als geen ander doorheen te slaan. Maak er wat moois van en laat je niet kennen! Een goede lach is een genot voor het leven.

Eline (van Ewijk) bedankt voor onze samenwerking op het ATGL-/- project. Hoewel er niet uitgekomen is wat we er in eerste instantie van verwacht hadden is het zeker wel een mooi project geworden. Ik hoop dat je de zwangerschapsstudie ook met veel plezier en succes zal afronden. Het was leuk om met je gewerkt te hebben en hoop dat je een mooi boekje in elkaar zal steken over een niet al te lange tijd. Ik zal deze in ieder geval met veel plezier lezen.

Madeleen, we startten ongeveer tegelijk en jij haalde iets eerder de eindstreep. Je bent een zeer gedreven hardwerkende onderzoeker en hoop dat je in Stockholm je dromen waar kan maken. Heel veel succes daar.

Lucas, je fysische kennis is zeker een grote meerwaarde binnen onze onderzoeksgroep en met de Acetylcarnitines heb je reeds mooie dingen gedaan. Heel veel succes de komende jaren met het afronden van je PhD.

Dennis, toen je hier begon als masterstudent waren we al meteen onder de indruk van je inzet en enthousiasme. Het feit dat je reeds bij Auwerx in het lab hebt mogen werken en een Kootstra beurs binnen wist te halen, kan alleen maar betekenen dat er nog veel mooie dingen op je liggen te wachten in de toekomst.

Anne, hoewel je helaas ver weg gezeteld bent, ben je zeker wel een van de meest actieve mensen in de SHOCk-groep. Niet alleen op de werkvloer, maar ook daarbuiten (en dan heb ik het niet enkel over het badmintonnen). Heel veel succes en plezier met je onderzoek en alle andere dingen die je ernaast nog doet!

Christine, alhoewel we niet lang/veel samengewerkt hebben, ging je reputatie je reeds voor. Lang voordat je bij ons begon had ik veel van je werk gelezen. Het is ongelofelijk wat je al bereikt hebt en het is dan ook fantastisch dat je Maastricht hebt gekozen om je ambities verder te verwerkelijken. Je werk-ethiek is inspirerend en ik hoop ook dat het opzetten van de technieken waarmee je bezig bent, spoedig zullen resulteren in elegante onderzoeken. Bedankt voor je inzet en aanvullingen bij het onderzoek en veel succes bij het waarmaken van je dromen.

Joris en Johanna, ondanks dat we nooit heel intensief samengewerkt hebben, waren jullie op de achtergrond wel altijd betrokken; voor de oxygraaf dan wel voor de qPcr en al het andere lab werk. Hoewel we het vaak vergeten is de expertise van mensen zoals 
jullie essentieel voor het uitvoeren van degelijk en deugdelijk wetenschappelijk onderzoek. Bedankt voor jullie werk en de kritische noot die jullie stelden bij het onderzoek. En heel veel geluk en liefde samen, ook met Nils en Naomi en de zwangershap!

Ook wil ik graag Esther Kornips en Gert Schaart bedanken die ook op de achtergrond bij de onderzoeken betrokken zijn geweest. Ik ben echt ongelofelijk dankbaar voor het werk dat jullie in de studies gestoken hebben; voor het snijden en kleuren van coupes en het doen van vele Western-blots en qPCrs. In grote onderzoeken en met het verloop van vele AIO's door de jaren heen, is het echt een luxe om experts zoals jullie in huis te hebben. Jullie werken altijd hard, klagen nooit en doen alles met een glimlach. Ik hoop dan ook dat jullie nog vele andere AIOs gelukkig mogen maken met mooie uitslagen en blotjes! Hierbij wil ik ook Dennis van Beurden bedanken, die helaas niet meer bij de Shock werkzaam is. Dennis bedankt!

Daarnaast ook bedankt aan alle oude SHOCk-leden die nu hun geluk ergens anders beproeven; Ronnie, Johan, Miranda, Ruth en Ellen!

Noud, ik ben je niet vergeten maar wilde je graag apart bedanken. Dit omdat je dubbele rol vervulde van zowel (ex-)middenlob-ber als (ex-)SHOCker. Jij mocht 4 jaar lang van 2 walletjes eten en wel de walletjes van zowel Ronald als Patrick. Dit zorgde ervoor dat jij in de middenlob gezeteld was, maar wel $50 \%$ van je tijd aan de SHOCk-groep besteedde. Bij plaatsgebrek aan de Zuid-kant van het gebouw, werd ik ook in de middenlob geplaatst (fysiek dan). Hier ben ik erg blij om, omdat ik in de middenlob heel warm werd ontvangen, o.a. door jou. Jij nam mij op sleeptouw naar alle clamps, oxygraaf metingen, VO2max testen, etc. Je vertelde vol enthousiasme over je onderzoek en beantwoorde al mijn (domme) vragen met plezier. Ook nam je me mee koffie drinken, waar wij vaak discussies hadden, waaronder over het rehydratie effect van Aquarius ter voorkoming van een kater. Helaas werd niet iedereen warm van onze 'Aquarius-studie', waardoor deze nooit doorgang heeft gevonden. Ik stel dan ook voor dat we dit onderzoek voortzetten op de avond van het promotiefeest en iedere feestganger Aquarius of een placebo mee naar huis geven. Dan zal eindelijk het mysterie rond de Aquarius-kater opgelost worden?! Bedankt voor alle behulpzaamheid en gezelligheid!

Daarnaast wil ik ook Sanne in het bijzonder bedanken. Hoewel we nooit op een studie samengewerkt hebben, was de sfeer altijd erg goed. Wij zijn dikke maatjes geworden dankzij het vele koffiedrinken (en ik drink niet eens koffie, kan je nagaan). Op de momenten waarop we even stoom moesten afblazen waren we er voor elkaar, wat af en toe wel even nodig was. Ook ben je vanaf het begin dol geweest op Bram (mijn zoontje, niet te verwarren met bovenstaande Bram). Dat was heel fijn, want zowel jij als Sabine wilden altijd wel even 
een oogje op hem houden als ik een afspraak had op vrijdag! Je bent een hele lieve, trouwe, betrouwbare, spontane en vooral ook erg vrolijke persoonlijkheid en ik ben dan ook erg blij dat jij mijn paranimf wil zijn. Hopelijk gaan we nog vaak cocktailtjes drinken!

Sabine, jou kan ik natuurlijk ook niet overslaan zonder een woordje te besteden aan alle gekkigheid die je mij de afgelopen 4 jaar bezorgde. Er zijn niet veel onderzoeken waar je aan mee kan doen als vrouw zijnde, maar bij jou mocht ik meedoen. Na maanden lang een dieet van enkel boter te volgen, zat ik braaf bij jou in het kamertje. Gelukkig waren er nog andere proefpersonen en werd het heel gezellig. Zo gezellig zelfs dat ik er mijn verjaardag doorbracht. Maar het was nog veel gezelliger om je collega en vriendin te zijn. We hebben veel avondjes lekker gegeten en geborreld (zonder botertjes). Tevens zijn we met zijn vieren (met Sanne en Yvonne) naar Brussel geweest. Een weekend om nooit te vergeten (en heb dan ook een vaag vermoeden dat ik er binnenkort weer aan herinnerd zal worden). Bedankt voor je steun en gezelschap en voor de goede zorgen voor Bram.

Natuurlijk ook dank aan alle andere (ex)middenlobbers: Yvonne, Leonie, Julia, Dorien, Sophie, Nina, Els, Nadine, Florence, Maurice, Ruth, Ronald, Jochem, Martine en Peter, bedankt voor de mooie tijd in de middenlob. Bedankt dat jullie me zo liefdevol opgenomen hebben in jullie clubje en dat het verbond is blijven bestaan ondanks de verhuizing naar niveau 0.

Natuurlijk wil ik ook mijn studenten bedanken die me tijdens hun bachelor/master/WESP stage geholpen hebben bij het onderzoek; Jim, Dennis, Inge, Stephanie, Nina en Tim. Ik hoop dat het jullie allemaal goed gaat en jullie allen een mooie carrière tegemoed gaan. Heel veel geluk voor de toekomst!

En dan natuurlijk onze rots in de branding, onze directe lijn naar de buitenwereld vanuit de dungeons (niv. 0.) van de Humane Biologie; de dames van het secretariaat! Claudia en Desiree; als vaste secretaresses weten jullie een vrolijke noot te geven aan alle saaie en serieuze zaken die geregeld moeten worden! Het is altijd een genot om even het secretariaat binnen te wippen en een borrel waar jullie bij zijn is werkelijk genieten! Yolanda, jij was een zeer goede toevoeging hierop, ik hoop dat je nog lang mag blijven. En natuurlijk ook dank aan Ilona en Shelly die ook geruime tijd het fort bewaakt hebben!

Daarnaast natuurlijk mijn dank aan alle overige collega's van de Humane Biologie en Bewegingswetenschappen. Guy V., bedankt voor de fijne samenwerking op de thyroid studie; jammer dat er weinig patiënten waren, maar het gaat vast goed komen met Evie op de studie. Heel veel succes met de opleiding tot chirurg! Guy P, bedankt burio en sterkte 
met de jaloezieën. Gijs, het is me een groot genoegen dat jij als opponent aanwezig zal zijn op mijn promotie. We hebben altijd zeer prettig samengewerkt en kijk dan ook al uit naar je vragen.

Voor iedereen die ik nog niet genoemd heb, bedankt voor het lenen van materialen dan wel het verlenen van hulp/expertise bij het onderzoek.

En als dat alles niet van toepassing is, dan bedankt voor de gezellige borrels, en Jos bedankt voor de goede organisatie van deze vrijdagmiddag borrels!

Paul, Loek en Laurens; bedankt voor de trouwe technische ondersteuning. Zonder jullie zou alles in het honderd lopen. Bedankt dat jullie altijd klaar staan en zorgen dat de getalletjes kloppen en de apparaten werken. Het wordt vaak vanzelfsprekend gevonden dat alles werkt, maar dat is het niet...

Tevens gaat mijn dank uit naar alle mensen waarmee we samengewerkt hebben. Op het CTMM project wil ik in het specifiek de projectleider Prof. Jan Glatz bedanken voor zijn commitment en inzet. Ook wil ik hierbij meteen Dr. Joost Luiken bedanken voor zijn bijdrage in dit project.

Dear Patricia Nunes; thank you very much for our nice collaboration. I have learned a lot from the tracer techniques that you have used in your studies. It was a privilege working with you. I hope you will finalise your work soon and I am sure that it will result in a very elegant thesis.

Prof. Heerschap en Prof. Tack van het 'Radboud Universitair Medisch Centrum Nijmegen', bedankt voor het delen van jullie visies en de aanvullingen op ons onderzoek. Het was een genoegen met jullie gewerkt te hebben en ik hoop dat jullie nog veel mooie dingen mogen bereiken binnen het metabole onderzoek.

Dear Frederic Girard of 'Spinnovation'; thank you very much for introducing us to the wonderful world of NMR. It was very nice working with you, I wish you and 'Spinnovation' all the best!

Also I would like to thank our colleagues from the Düsseldorf Diabetes Center in Germany. Professor Roden, it has been an honour working with you, as you set a great example for all clinical researchers. You also thought me that sometimes the results are more exiting when unexpected. I wish you all the best and hopefully we will meet again!

Dear Dr. Szendrodi, dear Julia, also special thanks to you as you have been quite involved in our studies, even whilst being busy in the clinic. I have always been amazed about how you 
and Prof. Roden manage to combine research and clinical work and hope that one day I will be able to do both as well. I wish you the best of luck with all your future endeavours! Also thanks to Allesandra Laufs, Jong-Hee Hwang (I hope I spelled it correctly this time) and Roshan Livingstone for your contribution to our joint efforts.

Ook wil ik al mijn nieuwe collega's op de afdeling radiologie bedanken. Ten eerste de laboranten van de MRI die de tijdens onze MRS metingen zich altijd vriendelijk en behulpzaam opgesteld hebben. Daarnaast ook dank aan het secretariaat/stafbureau en Prof. Wildberger voor het mogelijk maken van de samenwerking tussen de Radiologie en Humane Biologie. Ook dank aan alle Radiologen, Radiologen in opleiding en overige laboranten voor jullie support bij toeleven naar de promotiedatum.

Natuurlijk wil ik ook al mijn vrienden bedanken. De dames, Hanneke, Lonneke, Irene, Marloes en Marta. Hoewel ons leven steeds drukker wordt, maken we gelukkig nog altijd tijd voor elkaar; ik hoop dat dit altijd zo zal blijven. Bedankt voor jullie steun en geduld met mij! Bedankt voor jullie interesse in mijn onderzoek, hoewel dit niet altijd in 'Jip-en-Janneke' taal is; ik zal mijn best doen dat het lekenpraatje wel begrijpbaar is. Marta veel succes 3 juli, Marloes heel veel succes met het afronden van je onderzoek! Hannes, Lonnes en Irene, ik ben blij en ook enigszins jaloers, dat jullie je roeping gevonden hebben en deze al (vrijwel) verwezenlijkt hebben; geniet ervan!

Ook dank aan alle mannen; Wenzel, Rob, Kevin, Sander, Frans, David en Yvo. Bedankt voor jullie altijd aanwezige interesse in het onderzoek, ook al begrepen jullie er niet altijd even veel van. Bedankt dat jullie altijd gebleven zijn wie jullie zijn. We hebben een geweldige tijd samen, en hopelijk blijft dat ook zo! Wenzel heel veel succes met de opleiding; hopelijk wordt je snel een collega in Maastricht. Rob en Kevin succes met jullie promotie en ambities in de geneeskunst. Sander, Frans, David en Yvo blijf vooral doen waar je goed in bent! (en dan bedoel ik niet bier drinken...)

Last but not least, wil ik natuurlijk mijn familie bedanken.

Pap, je bent altijd een groot voorbeeld voor me geweest. Je kracht van het logisch redeneren, je ongelofelijke volharding, inzet en je oprechtheid sieren je. Dat ik nu net zoals jij een promotie traject heb afgelegd vind ik geweldig! Je was een van de weinigen die echt de moeite genomen had op mijn eerste publicatie te lezen,. Bovendien kon je er ook nog 
wat zinnigs van maken! Ik hoop dat jij net zo trots op mij bent als ik op jou! Bedankt dat je er altijd voor me bent.

Mam, bedankt dat je altijd interesse hebt in wat ik doe, ook al denk je er soms meer over te weten dan ik. Bedankt dat je altijd een steun bent, of het nou om stellingen gaat of om het passen op Bram, ik kan altijd op je rekenen. Je onbaatzuchtigheid en kritische blik sieren je. Je wil het beste voor ons en wij ook voor jou. Bedankt voor je steun bij mijn promotietraject, de keren dat je voor mij als proefpersoon in de scanner hebt gelegen, voor de documenten die je gelezen hebt, voor de steun met Bram en met ons nieuwe huisje. We zeggen het misschien niet vaak genoeg, maar we zijn trots op je!

Natuurlijk ook bedankt aan mijn grote broer Henk en zijn vriendin Irma. Ook al hebben jullie waarschijnlijk weinig meegekregen van mijn promotie onderzoek, zijn jullie wel altijd geïnteresseerd en begaan. Bedankt!

Natuurlijk ook dank aan mijn (kleine) broertje Frans. Ook al woon je nu ver weg, je bent altijd razend benieuwd en trots op je zus. Soms zo trots dat ik in jou verhalen ineens cum laude ben afgestudeerd i.p.v. gewoon met een 8 gemiddeld, maar alla, het is je vergeven,... Ik hoop dat alles goed met je gaat en dat je weet dat ik ook heel trots ben op jou!

Dan natuurlijk ook veel dank aan mijn schoonfamilie. Al zijn Lars en ik niet getrouwd, ben ik wel altijd liefdevol opgenomen in de familie en voelt het net als thuis (maar dan dichterbij)! Lillian, super bedankt voor de open ontvangst in de familie en alle goede zorgen van de afgelopen jaren. Het is een grote steun en toeverlaat om te weten dat u er altijd voor ons bent, zowel voor mij als voor Lars en Bram. U staat altijd klaar om een handje toe te steken en weet de zaken altijd goed te regelen voor ons. Al is het onderzoek en het Academische leven soms wat ingewikkeld, $u$ vindt het altijd erg interessant. Ik vind het dan ook erg leuk dat u graag op de hoogte blijft van de gang van zaken. Ook naast het werk vindt u ontspanning in weekenden en op de feestdagen erg belangrijk; we kunnen altijd terecht bij u! Bedankt! Bob, u staat altijd voor iedereen klaar; pech met de fiets of de auto, autorijles, een huis verbouwen, op de kleinkinderen passen, je kan het zo gek niet bedenken of u staat voor ons klaar. $U$ doet alles met een glimlach en dat siert $u$. Uw goedlachsheid en verhalen doen de mensen stralen, hopelijk mogen we nog lang van u glimlach genieten!

Marco, Vivian, Valerie, Sophie, Bjorn, Wendy, Tren en Milan bedankt dat jullie er zijn! Als we dakloos waren gaven jullie ons onderdak. Als het lekker weer is kunnen we bij jullie terecht. Het is heel fijn om een leuke schoonfamilie te hebben en zeker als ze zo dichtbij wonen! Hopelijk kunnen we nog lang van alle goede dingen in het leven genieten en carnavallen totdat we erbij neervallen!

Ook wil ik graag Marco's Wielershop bedanken. Dankzij Marco's Wielershop fiets ik al jaren veilig op en neer naar mijn werk op de mooiste fietsen van Maastricht. Bedankt voor de goede service en goede zorgen! 
Lars, zonder jou kan ik me de afgelopen 4 jaar (of eigenlijk 8 jaar) niet voorstellen. Ongelofelijk bedankt dat jij er bent. Ik houd van je en zal altijd van je houden. Dankzij jou is het me gelukt alles met een goed resultaat af te ronden, maar daarnaast ook een sociaal leven te behouden. Met jou is het fijn en rustig thuis komen (en dan ook nog eens in een fantastisch mooi huis met een prachtige tuin).

Ook als partner ben je er altijd voor me geweest. Jij remt me af als ik soms doordraaf. Jij spoort me aan als ik dingen nalaat. Jij doet me realiseren dat er tijd is om te werken en dat er tijd is om thuis te zijn. Bedankt voor je steun en hulp bij mijn promotie onderzoek, maar vooral bedankt dat jij ook mijn partner wil zijn. Bedankt dat je me mijn dromen laat verwerkelijken en dat je achter me staat bij moeilijke beslissing, maar wel kritisch blijft. En bedankt voor het aller mooiste in mijn/ons leven, bedankt voor Bram.

Bram, je bent nu nog te klein maar mocht je dit ooit lezen als je later groot bent, mama houdt van je, je bent het mooiste wat ik ooit heb gemaakt en hoop dat je lang gelukkig en gezond mag leven. 


\section{About the author}

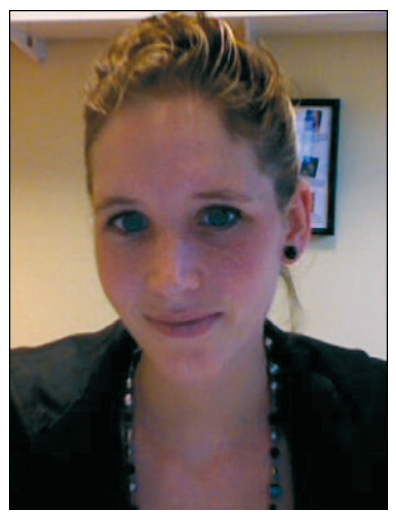

Tineke van de Weijer (MD) was born on the 31st of August 1984 in Eindhoven, the Netherlands. She studied Medicine at Maastricht University. During her studies she was active in several educational organs (amongst which the faculty board advisory committee, education platform and the medical student association M.F.V. Reflex, nowadays M.S.V. Pulse) and followed an International Trajectory (ITG). During this trajectory she did an extracurricular course on Tropical medicine, a minor on 'Globalisation and Inequality' and a course on 'Health Services Management' at the University of Birmingham (UK). During her clerkships she joined the department of 'Emergency Medicine' at the Princess Alexandra Hospital in Brisbane Australia and did research on epigenetic gene regulating pathways in drosophila at the 'Université de Pierre et Marie Curie' under the supervision of Dr. Frederique Perronet. Finally she received her medical degree in 2008.

In 2008 she received a 'Kootstra Talent Fellowship' from Maastricht University and started a PhD program at this University. In January 2009 she officially started as a PhD student at the department of Human Biology at Maastricht University. The research project was initially entitled 'Cardiac lipid accumulation and mitochondrial function' and resulted in this PhD thesis. After finalizing the project in februari 2013, she started as a resident at the department of Radiology at the Maastricht University Medical Center. Hopefully this will be the start of a new clinical research collaboration that will translate into a bench to bed-side story... 


\section{Over de auteur}

Tineke van de Weijer (MD) werd geboren op 31 augustus 1984 in Eindhoven. Ze studeerde geneeskunde aan de Universiteit Maastricht. Gedurende haar opleiding was ze actief in verschillende verenigingen en adviesorganen, waaronder de faculteitsraad, het onderwijsplatform en de Medische Studenten Vereniging 'Reflex' (tegenwoordig beter bekend als 'MSV Pulse'). Daarnaast volgde ze het internationale traject van de Geneeskunde opleiding en deed daarbij een extracurriculaire cursus in Tropische geneeskunde, een 'minor' in 'Globalisation and inequality' en een cursus over 'Health Services Management' aan de Universiteit van Birmingham (Engeland). Tijdens haar co-schappen werkte ze onder andere op de eerste hulp (department of 'Emergency Medicine') aan het 'Princess Alexandra Hospital' in Brisbane (Australie). Voor haar wetenschapsstage deed ze onderzoek naar epigenetische regulerende signaleringscascaden in fruitvliegjes (drosophila) aan de 'Université de Pierre et Marie Curie' in Parijs (Frankrijk) onder supervisie van Dr. Frederique Perronet. Uiteindelijk ronde ze haar medische opleiding af in 2008.

In 2008 ontving zee en beurs van de Universiteit Maastricht genaamd de 'Kootstra Talent Fellowship'. Hiermee kon ze een promotietraject in gang zetten. In januari 2009 startte ze dan ook officeel als promovendus a an de vakgroep humane biologie aan de Universiteit Maastricht. Het onderzoeksproject was initieel getiteld 'Cardiale vetstapeling en mitochondriele functie' en resulteerde uiteindelijk in deze thesis. Na afronding van het promotie traject in februari 2013 startte ze als arts-assistent Radiologie in het Academisch Ziekenhuis Maastricht. 

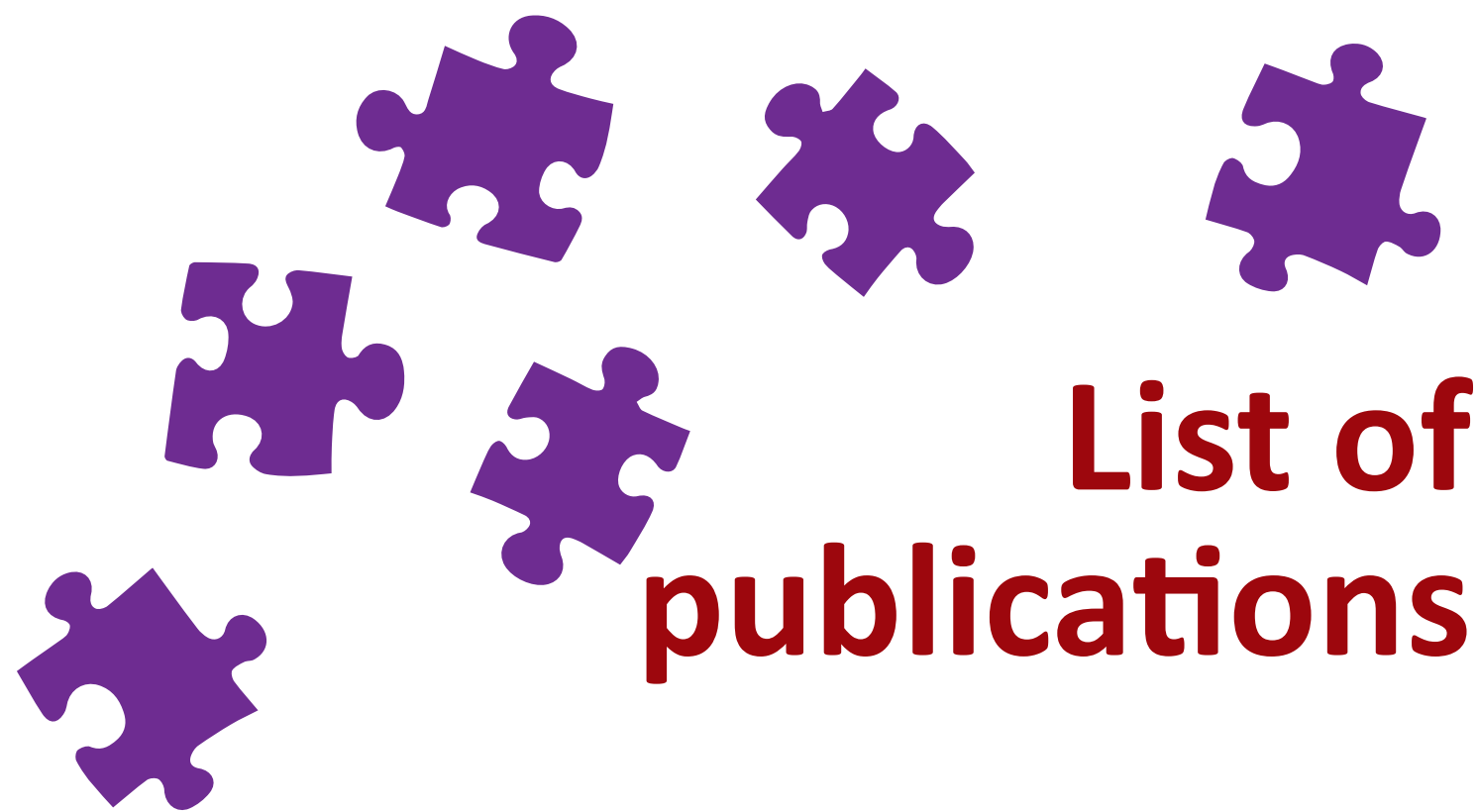

\section{publications}




\section{Published papers}

- Meex RC, Schrauwen-Hinderling VB, Moonen-Kornips E, Schaart G, Mensink M, Phielix E, van de Weijer T, Sels JP, Schrauwen P, Hesselink MK; Restoration of muscle mitochondrial function and metabolic flexibility in type 2 diabetes by exercise training is paralleled by increased myocellular fat storage and improved insulin sensitivity; Diabetes. 2010 Mar;59(3):572-9. doi: 10.2337/db09-1322.

- $\quad$ Bilet L, van de Weijer T, Hesselink MK, Glatz JF, Lamb HJ, Wildberger J, Kooi ME, Schrauwen P, Schrauwen-Hinderling VB; Exercise-induced modulation of cardiac lipid content in healthy lean young men; Basic Res Cardiol. 2011 Mar;106(2):307-15. doi: 10.1007/s00395-010-0144-x. Epub 2010 Dec 23.

- Schrauwen-Hinderling VB, Meex RC, Hesselink MK, van de Weijer T, Leiner T, Schär M, Lamb HJ, Wildberger JE, Glatz JF, Schrauwen P, Kooi ME; Cardiac lipid content is unresponsive to a physical activity training intervention in type 2 diabetic patients, despite improved ejection fraction; Cardiovasc Diabetol. 2011 May 26;10:47. doi: 10.1186/1475-2840-10-47.

- $\quad$ van de Weijer T, Schrauwen-Hinderling VB, Schrauwen P; Lipotoxicity in type 2 diabetic cardiomyopathy; Cardiovasc Res. 2011 Oct 1;92(1):10-8. doi: 10.1093/cvr/cvr212. Epub 2011 Jul 29. Review.

- Haemmerle G, Moustafa T, Woelkart G, Büttner S, Schmidt A, van de Weijer T, Hesselink M, Jaeger D, Kienesberger PC, Zierler K, Schreiber R, Eichmann T, Kolb D, Kotzbeck P, Schweiger M, Kumari M, Eder S, Schoiswohl G, Wongsiriroj N, Pollak NM, Radner FP, Preiss-Landl K, Kolbe T, Rülicke T, Pieske B, Trauner M, Lass A, Zimmermann R, Hoefler G, Cinti S, Kershaw EE, Schrauwen P, Madeo F, Mayer B, Zechner R; ATGL-mediated fat catabolism regulates cardiac mitochondrial function via PPAR- $\alpha$ and PGC-1; Nat Med. 2011 Aug 21;17(9):1076-85. doi: 10.1038/nm.2439.

- Timmers S, Konings E, Bilet L, Houtkooper RH, van de Weijer T, Goossens GH, Hoeks J, van der Krieken S, Ryu D, Kersten S, Moonen-Kornips E, Hesselink MK, Kunz I, SchrauwenHinderling VB, Blaak EE, Auwerx J, Schrauwen P; Calorie restriction-like effects of 30 days of resveratrol supplementation on energy metabolism and metabolic profile in obese humans; Cell Metab. 2011 Nov 2;14(5):612-22. doi: 10.1016/j.cmet.2011.10.002. 
- $\quad$ van de Weijer T, van Ewijk PA, Zandbergen HR, Slenter JM, Kessels AG, Wildberger JE, Hesselink MK, Schrauwen P, Schrauwen-Hinderling VB, Kooi ME; Geometrical models for cardiac MRI in rodents: comparison of quantification of left ventricular volumes and function by various geometrical models with a full-volume MRI data set in rodents; Am J Physiol Heart Circ Physiol. 2012 Feb 1;302(3):H709-15. doi: 10.1152/ ajpheart.00710.2011. Epub 2011 Nov 18.

- $\quad$ Nunes PM, van de Weijer T, Veltien A, Arnts H, Hesselink MK, Glatz JF, Schrauwen P, Tack CJ, Heerschap A; Increased intramyocellular lipids but unaltered in vivo mitochondrial oxidative phosphorylation in skeletal muscle of adipose triglyceride lipase-deficient mice; Am J Physiol Endocrinol Metab. 2012 Jul 1;303(1):E71-81. doi: 10.1152/ ajpendo.00597.2011. Epub 2012 Apr 10.

- van de Weijer T, Sparks LM, Phielix E, Meex RC, van Herpen NA, Hesselink MK, Schrauwen P, Schrauwen-Hinderling VB; Relationships between mitochondrial function and metabolic flexibility in type 2 diabetes mellitus; PLoS One. 2013;8(2):e51648. doi: 10.1371/journal.pone.0051648. Epub 2013 Feb 13.

- van de Weijer T, Havekes B, Bilet L, Hoeks J, Sparks L, Bosma M, Paglialunga S, Jorgensen J, Janssen MC, Schaart G, Sauerwein H, Smeets JL, Wildberger J, Zechner R, Schrauwen-Hinderling VB, Hesselink MK, Schrauwen P; Effects of bezafibrate treatment in a patient and a carrier with mutations in the PNPLA2 gene, causing neutral lipid storage disease with myopathy; Circ Res. 2013 Mar 1;112(5):e51-4. doi: 10.1161/ CIRCRESAHA.113.300944.

\section{Submitted}

- van de Weijer T, Phielix E, Bilet L, Laufs A, Livingstone R, Nowotny P, Sparks LM, Paglialunga S, Szendroedi J, Hwang J, Schrauwen-Hinderling VB, Hesselink MKC, Roden $\mathrm{M}$, Schrauwen P; Treatment with Acipimox fails to improve insulin sensitivity and may impair cardiac function in non-insulin dependent type 2 diabetes mellitus

- $\quad$ Lindeboom L, Brouwers BMW, van de Weijer T, Hesselink MKC, Wildberger J, Kooi ME, Schrauwen P, Schrauwen-Hinderling VB; Long TE ${ }^{1} \mathrm{H}-\mathrm{MRS}$ acquisition for non-invasive dynamic monitoring of acetylcarnitine levels in vivo 


\section{With special thanks to:}

\section{Maastricht UMC+}

azM
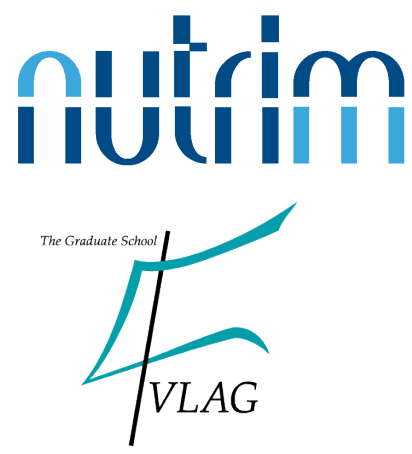

ctmm

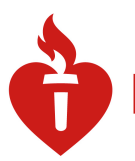

gesubsidieerd door

Hartstichting
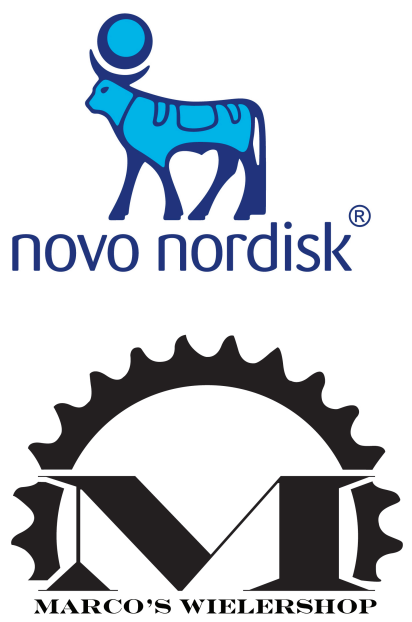

Maastricht University Medical Center+

NUTRIM

School for Nutrition, Toxicology and Metabolism

Graduate School VLAG

Center for Translational Molecular Medicine

Dutch Heart Foundation

Novo Nordisk ${ }^{\circledR}$

Marco's Wielershop, Maastricht. 